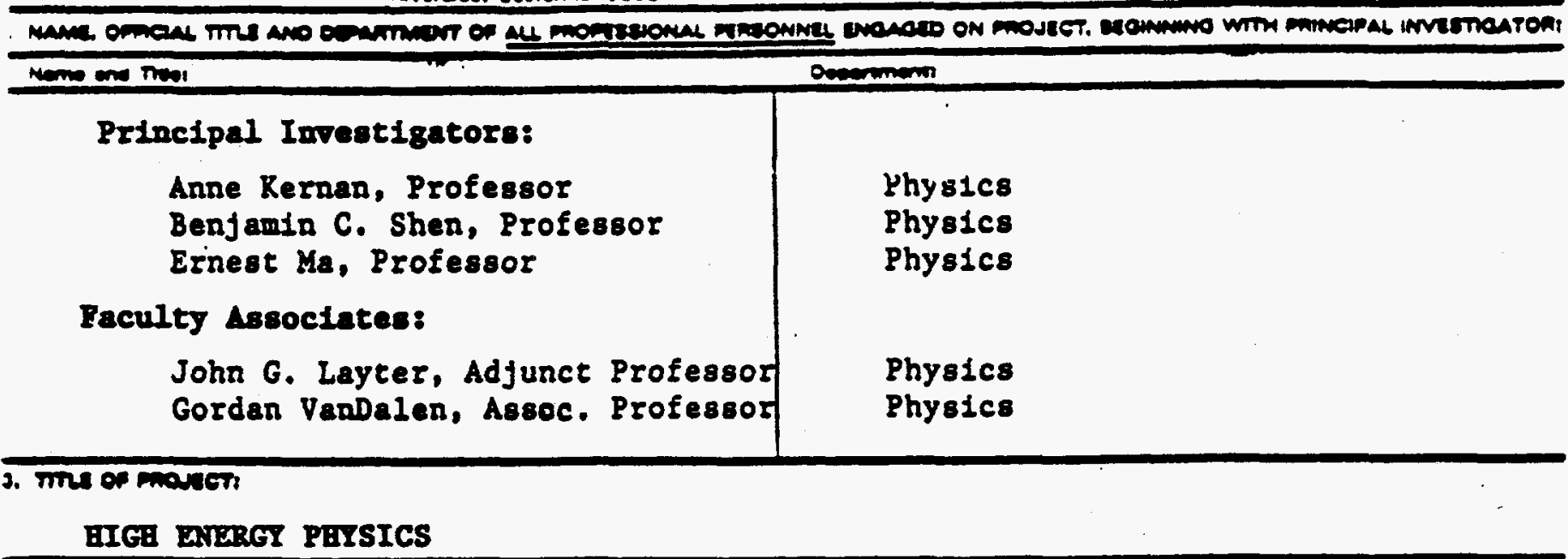

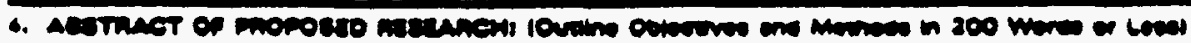

Hadron collider studies will focus on: (i) the search for the top quark with the newly installed DO detector at the Fermilab Tevatron collider, (ii) the upgrade of the DO detector to match the new main injector luminosity and (iii) $R \& D$ on silicon microstrip tracking devices for the SSC. High statistics studies of $Z^{\circ}$ decay will continue with the OPAL detector at LEP. These studies will include a direct measurement of $Z$ decay to neutrinos, the search for Higgs and heavy quark decays of Z. Preparations for the Large Scintillation Neutrino Detector (LSND) to measure neutrino oscillations at LAMPF will focus on data acquisition and testing of photomultiplier tubes.

In the theoretical area E. Ma will concentrate on mass-generating radiative mechanisms for light quarks and leptons in renormalizable gauge field theories. J. Wudka's program includes a detailed investigation of the magnetic-flip approach to the solar neutrino problem. 


\section{HIGH ENERGY PHYSICS AT UCR}

Table of Contents

\section{PHYSICS RESEARCH}

\section{A. Experimental High Energy Physics 1}

1. Hadron Collider Physics 1

a) Overview/Personnel/Talks/Publications 1

b) D-Zero Proton-Antiproton Interactions at $2 \mathrm{TeV} \quad 10$

c) Detector Development 27

d) Nucleon Structure Functions 32

2. $\mathrm{e}^{+} \mathrm{e}^{-}$Collider Physics and Neutrino Physics 38

a) Overview/Personnel/Talks/Publications 38

b) TPC/2 $\gamma$ at PEP 53

c) OPAL at LEP 60

d) Neutrino Physics at LAMPF 126

e) Future Directions 137

B. Theory 143

a) Introduction 143

b) Research Program

c) Publications 146

d) Travel and Consultants 149

e) Personnel and needs 150

f) Future Research Plans 150

g) Recent Research Activity (J. Wudka) 155

II. HIGH ENERGY PHYSICS COMPUTING AT UCR 161

$\begin{array}{ll}\text { III. BUDGET } & \text { B1-B48 }\end{array}$

IV. VITAE AND PUBLICATIONS V1-V63

DISCLAIMER

This report was prepared as an account of work sponsored by an agency of the United States Government. Neither the United States Government nor any agency thereof, nor any of their employees, makes any warranty, express or implied, or assumes any legal liability or responsibility for the accuracy, completeness, or usefulness of any information, apparatus, product, or process disclosed, or represents that its use would not infringe privately owned rights. Reference herein to any specific commercial product, process, or service by trade name, trademark, manufacturer, or otherwise does not necessarily constitute or imply its endorsement, recommendation, or favoring by the United States Government or any agency thereof. The views and opinions of authors expressed berein do not necessarily state or reflect those of the United States Government or any agency thereof. 


\section{DISCLAMIER}

Portions of this document may be illegible in electronic image products. Images are produced from the best available original document. 
$1 a$. HADRON COLLIDER PHYSICS OVERVIEW

Installation of D-Zero is now in progress and we anticipate first proton-antiproton interactions in Summer 1991. With the highest energy collisions in the world the Tevatron collider will dominate high energy physics through the nineties. As the luminosity increases the mass range for supersymmetric particle and new vector boson searches will extend to the $500 \mathrm{GeV}$ region. And, most important, only Fermilab offers the opportunity to find the top quark in the nineties.

With data taking imminent we will emphasize preparations for physics analysis. Our other major focus will be the upgrade of the D0 tracking system to match the 1995 luminosity upgrade. We are also committed to preparations for the SSC at the end of this decade.

The $\mathrm{D} 0$ integrated high voltage system for which we took a major responsibility is now fully realised. After three years and four generations of prototypes the design is finalised and the system is expected to be fully operational in Spring 1991. It will be the largest and most advanced integrated system of its kind in operation. Several of the elements developed for this system have been adopted for commercial production.

We will be active in the commissioning of the $D 0$ muon detector. At UCR we have just completed testing and modifying the full complement of Module Address Cards for the muon trigger electronics. The test setup has now been moved to Fermilab where final modifications of the boards will be carried out by UCR personnel. We are also developing the off-line reconstruction package for the muon detector.

In 1991 software activities will expand in preparation for physics analysis. This will require a broad upgrade of the high energy computing infrastructure at UCR for which a proposal is in preparation. We will focus on the search for top and on W,Z electroweak studies. High statistics Monte Carlo event generation will be necessary to investigate both triggering strategies and backgrounds for the various decay modes of top. 
Since early $1989 \mathrm{~S}$. Wimpenny and J. Ellison have been developing with university funds a well-equipped facility for $R \& D$ on silicon microstrip tracking devices. Wimpenny and R. Lipton (Fermilab) are coordinating design work on a silicon microstrip detector for the DO upgrade. Wimpenny and Ellison are also members of the SDC collaboration and are participating in the recently funded collaboration for R\&D on a large silicon tracking system for the SSC. J. Ellison is co-chair (along with Prof. Ohsugi of Hiroshima University) of the Double-sided Detectors sub-group of this collaboration. On July 15,16 this year thirty members of the SSC Silicon Tracking Subsystem group attended a collaboration meeting hosted by J. Ellison at UCR. Work has started on radiation hardness studies at LANL and on the evaluation of double-sided microstrip devices.

In the personnel area A. Klatchko, a recent graduate of Tel-Aviv University filled the vacant postdoctoral position. D. Smith, Associate Research Physicist, will leave August 1990 to assume a faculty position at Embry-Riddle Aeronautical University. In his place we plan to appoint a post-doc who will concentrate on D0/SSC detector development. D. Joyce and M. Lindgren, our last UAl graduate students, receive their Ph. D's in Fall 1990.

During the last year S. Wimpenny presented invited talks at the EPS Conference, Madrid, September 1989 and at the Fermilab Workshop on Structure Functions and Parton Distributions, April 1990. Graduate student M. Ikeda presented his thesis work at the 8th Topical Workshop on Proton-Antiproton Collider Physics, Castiglione, Italy, September 1989, and M.-J. Yang described the D-Zero High Voltage System at the 2nd International Conference on Advanced Technology and Particle Physics, Como, July 1990. A. Kernan was elected to the Executive Committee of the SSC Users' Organization. She recently completed with N. Ellis of Birmingham University a Physics Report on "Heavy Quark Production at the CERN pp Collider". 
PERSONNEL - HADRON COLLIDER PHYSICS

\section{Ph.D.'s}

J. Ellison $\quad$ : $\quad$ assistant research physicist

A. Kernan : professor

A. Klatchko : post graduate researcher

S.J. Wimpenny : associate professor

M.-J. Yang ： post graduate researcher

To be named : : post graduate researcher

Graduate Students
K. Bazizi
R. Hall
D. Franks

To be named

Undergraduate Students

T. Fahland

S. Jerger

C. Litzke 


\section{INVITED TALKS - HADRON COLLIDER TASK 1989 -}

1. A. Kernan, "Problems and Opportunities for the US HEP Community Overseas". Talk to HEPAP sub-panel on future directions in HEP, Hilton Head, S.C., Jan. 18, 1989.

2. A. Kernan, "A Survey of Discoveries Made at the CERN Proton-Antiproton Collider". Colloquium, California State University, Los Angeles, Jan. 25, 1989.

3. D. Joyce, "Silicon Strip Detectors: An SFSU Alumnus Reports from CERN. Colloquium - San Francisco State Univ., May 15, 1989.

4. M. Yang, "The Design of the D-Zero High Voltage System". Colloquium, Fermilab, May 7, 1989.

5. D. Smith, "Identification of b-jets with a Silicon Vertex Detector in D-Zero". Seminar, D-Zero Group Meeting, Fermilab, April 6, 1989.

6. D. Smith, "Survey of High Energy Physics". Colloquium, UC Riverside, Physics/Science Teachers, Riverside, San Bernardino Counties, July 18, 1989.

7. M. Ikeda, "The Charm Content of Jets at the CERN pp Collider". 8th Topical Workshop on Proton-Antiproton Collider Physics, Castiglione, Italy, Sept. 1989.

8. D. Smith, "Identification of b-jets with a Silicon Vertex Detector in D-Zero". Talk presented at the the Breckenridge Workshop on Collider Physics at Fermilab in the 1990's, August 1989.

9. S. Wimpenny, "D* Production in Jets at the CERN Proton-Antiproton Collider". Invited talk presented at European Physical Society Conference on High Energy Physics, Madrid, Spain, Sept. 1989.

10. J. Ellison, "Simulation of a Silicon Vertex Detector in DO". Seminar, D-Zero Group Meeting, Fermilab, Dec. 8, 1989.

11. S.J. Wimpenny, "A New Analysis of the EMC Hydrogen Data". Talk presented at the Workshop on Structure Functions and Parton Distributions, Fermilab, 26-28th April, 1990.

12. R. Hall, "The D0 Detector at the Fermilab p-p- Collider". Colloquium, California State University, Fresno, April 30, 1990. 
Invited Talks - Hadron Collider Task

Page 2

13. D. Smith, "The Search for Top at the Fermilab pp Collider". Colloquium, California State University, San Bernardino, April 5, 1990.

14. M.-J. Yang, "The D-Zero High Voltage System". Invited talk presented at the 2nd International Conference on Advanced Technology and Particle Physics, Como, Italy, June 11-15, 1990.

15. J. Ellison, "Results of Simulation on a Silicon Vertex Detector in D0". Talk presented at the 1990 Summer Study on High Energy Physics - Research Directions for the Decade, Snowmass CO, June 25-July 13, 1990.

16. A. Kernan, "Muon Triggering at the CERN Proton-antiproton Collider". Talk presented at the 1990 Summer Study on High Energy Physics - Research Directions for the Decade, Snowmass CO, June 25-July 13, 1990. 


\section{PUBLICATIONS - HADRON COLLIDER TASK}

1989 -

1. "The Hadron and Electron Response of Uranium/Liquid Argon Calorimeter Modules for the D $\varnothing$ Detector", S.J. Wimpenny, Proceedings of the Int. Conf. on Advanced Technology and Particle Physics, Como, Italy, June 1988, NIM A279, 107-113, 1989.

2. "The Computer Controlled VME High Voltage System for the D-Zero Detector", S.J: Wimpenny, Proceedings of the International Conf. on Advanced Technology and Particle Physics, Como, Italy, June 1988, NIM, A279, 359-363, 1989.

UCR authors: K. Roberts, S.J. Wimpenny

3. "Silicon Detector Tests with the RAL Microplex Readout Chip", Proceedings of the Int. Conf. on Advanced Technology and Particle Physics, Como, Italy, June 1988, Nucl. Instrum. Methods, A279, Nos. 1,2, 189-194 (1989).

UCR authors: J. Ellison, D. Joyce, J.-P. Merlo, K. Morgan, D. Smith.

4. "Silicon Detector Tests with the RAL Microplex Readout Chip", D. Joyce, Proceedings of the 7th Topical Workshop on Proton-Antiproton Collider Physics June 1988 - Fermilab, 464-481, (1989).

5. "Results on Inclusive Physics from UA1", S.J. Wimpenny, Proceedings of the APS-DPF Meeting, Univ. of Conn., Storrs, August 14-18, 1988, World Scientific Publishing, 458-463 (1989).

6. "Heavy Flavor Production in UA1", S.J. Wimpenny, Proceedings of the APS-DPF Meeting, Univ. of Conn., Storrs, August 14-18, 1988 World Scientific Publishing, 488-492 (1989).

7. Measurement of the Bottom Quark Production Cross Section in Proton-Antiproton Collisions at $\sqrt{s}=0.63 \mathrm{TeV} "$, (UA1 Collaboration), Phys. Lett. 213B, 405-412 (1989).

UCR authors: M. Ikeda, D. Joyce, A. Kernan, M. Lindgren, J.P. Merlo, K. Morgan, I. Sheer, D. Smith, S.J. Wimpenny.

8. "Measurements of the $u$ Valence Quark Distribution Function in the Proton and $u$ Quark Fragmentation Functions", (European Muon Collaboration), Nucl. Phys. B321, 541-560 (1989).

UCR author: S.J. Wimpenny 
9. "Studies of Intermediate Vector Boson Production and Decay in UA1 at the CERN Proton-Antiproton Collider", (UA1 Collaboration), Zeit. Phys. C44, 15-61 (1989). UCR authors: R. Frey, M. Ikeda, D. Joyce, A. Kernan, M. Lindgren, J.P. Merlo, K. Morgan, J. Ransdell, I. Sheer, D. Smith, S.J. Wimpenny.

10. "Hadron and Electron Response of Uranium/Liquid Argon Calorimeter Modules for the Dø Detector", The Dø Collaboration, Nucl. Instrum. Methods, A280, 36-44 (1989).

UCR authors: K. Roberts, S.J. Wimpenny.

11. "The $\mathrm{D}^{*}$ Content of Jets in $\mathrm{p} \overline{\mathrm{p}}$ Interactions at the CERN Collider", M. Ikeda, Ph.D. Thesis, University of California, Riverside, CA., June 1989.

12. "A Study of the General Characteristics of Proton-Antiproton Collisions at $V_{s}=0.2$ to $0.9 \mathrm{TeV}$ ", (UA1 Collaboration), accepted by Nucl. Phys. B, CERN-EP/89-85, 29 pp. (1989).

UCR authors: M. Ikeda, D. Joyce, A. Kernan, M. Lindgren, K. Morgan, D. Smith.

13. "Prospects for Top at the Tevatron Collider in the 1990's", D. Smith. Proceedings of the Workshop on Physics at Fermilab in the 1990's, Breckenridge, Aug. 15-24, 1989.

14. "An Investigation of the Spin Structure of the Proton in Deep Inelastic Scattering of Polarised Muons on Polarised Protons", (European Muon Collaboration), Nucl. Phys. B328, 1-35 (1989).

UCR author: S.J. Wimpenny

15. "High Luminosity Muon Scattering at FNAL - Report of the Study Group on Future Muon Scattering Experiments at the Workshop on Physics at Fermilab in the 1990 's". Proceedings of the Workshop on Physics at Fermilab in the 1990's, Breckenridge, Aug. 15-24, 1989, Fermilab-Conf-90/39, UCR/DIS-90-01. UCR author: K. Bazizi

16. "Bose-Einstein Correlations pip interactions at $\sqrt{ } s=0.2$ to $0.9 \mathrm{TeV}$ ", (UA1 Collaboration), submitted to Phys. Lett. B, CERN-EP/89-71, (1989). UCR authors: M. Ikeda, D. Joyce, A. Kernan, M. Lindgren, K. Morgan, D. Smith.

17. "Heavy Quark Production at the CERN pp Collider", A. Kernan, N. Ellis, UCR/HEP-A1/89-10, October, 1989, (to appear in Physics Reports).

18. "The Charm Content of Jets at the CERN po Collider", M. Ikeda, Proceedings of the 8th Topical Workshop on Proton-Antiproton Collider Physics, Castiglione, Italy, Sept. 1989. 
19. "D* Production in Jets at the CERN Proton-Antiproton Collider", S.J. Wimpenny, Proceedings of the European Physical Society Conference on High Energy Physics, Madrid, Spain, Sept. 1989, UCR/UA1-89-10, 10 pp. (1989).

20. "Comparison W and Direct Photon Cross-Sections at Large Transverse Momentum", (UA1 Collaboration), submitted to Zeit. Phys. C, CERN-EP/89-138, 19 pp. (1989).

UCR author: M. Lindgren

21. "Influence of the Electric Field on Compensation in a Uranium/Tetramethyl Pentane Hadron Calorimeter", (UA1 Collaboration), submitted to Nucl.Instrum. Methods, CERN-EP/89-160, 13 pp. (1989)

UCR author: $M$. Lindgren

22. "Search for New Heavy Quarks in Proton-Antiproton Collisions at $\sqrt{s}_{\mathbf{s}}=0.63$ TeV", (UA1 Collaboration), submitted to Z. Physics C, CERN-EP/90-57, 25 pp. (1990)

UCR author: M. Lindgren

23. "Measurements of the Nucleon Structure Function in the Range $0.002<x, 0.17$ and $0.2<Q^{2}<8 \mathrm{GeV} / \mathrm{c}^{2}$ in Deuterium, Carbon and Calcium", (European Muon Collaboration), Nucl. Phys. B333, 1-47 (1990).

UCR author: S.J. Wimpenny

24. "Study of the $D^{*}$ Content of Jets at the CERN $\mathrm{pp}$ Collider", (UA1 Collaboration), CERN-EP/90-61, (accepted by Phys. Lett.), 16 pp., (1990).

UCR authors: M. Ikeda, D. Joyce, A. Kernan, M. Lindgren, S.J. Wimpenny.

25. "A Comparison of the Forward Produced Hadronic Muon Interaction on Nuclear Targets and Deuterium", (European Muon Collaboration), (submitted to Zeit. Phys. C), 36 pp. (1990).

UCR author: S.J. Wimpenny

26. "Simulation of a Silicon Vertex Detector in the DO Experiment", J. Ellison, A. Kernan and D. Smith, D0 Note 975, to be submitted to Nucl. Instrum. Methods, 1990.

27. "The D-Zero High Voltage System", M. J. Yang. Invited talk presented at the 2nd International Conference on Advanced Technology and Particle Physics, Como, Italy, June 11-15, 1990. To be published in conference proceedings.

28. "Results of Simulation on a Silicon Vertex Detector in D0", J. Ellison. Talk presented at the 1990 Summer Study on High Energy Physics - Research Directions for the Decade, Snowmass CO, June 25 - July 13, 1990. To appear in proceedings. 


\section{REPORTS/NOTES - HADRON COLLIDER TASK}

1989 -

1. "Vertex Drifter Chamber Cabling: - Power Supply to Detector Map, S. J. Wimpenny, Dø Note 698.

2. "Installing the DØ Magnetic Field Maps into ZEBRA banks, MMAP", D $\varnothing$ Note 812, K. Bazizi, D. Smith, (1989).

3. "Identification of b-Jets in Dø with a Barrel Silicon Vertex Detector", D. Smith, Dø Note 830, (1989).

4. "A Comparison of the RADEMC and Bardin-Shumeiko Radiative Correction Programs", C. Lietzke, S.J. Wimpenny, UCR/DIS-89-06, September, 1989.

5. "A Study of the Properties of the Ratio $R=\sigma_{L} / \sigma_{T}$ ", S. J. Wimpenny, K. Bazizi, UCR/DIS-90-04, May (1990).

6. "Convenor's Summary for Silicon Vertex Detector Working Group + Transparencies from Summary Presentation on 6-13-90", S.J. Wimpenny, UCRD0/90-02, (1990).

7. "A New Analysis of the European Muon Collaboration Hydrogen Data", S.J. Wimpenny and K. Bazizi, talk presented at Workshop on Hadron Structure Functions and Parton Distributions, Fermilab, II, 26-28 April 1990, UCR/DIS90-05, 8 pp. (1990). 
1 b. D-ZERO : PROTON-ANTIPROTON INTERACTIONS AT 2 TeV

\section{Introduction}

Riverside joined the D-Zero collaboration at Fermilab in late 1986. Our work to date spans four main areas: the implementation of the high voltage system, studies for a silicon microstrip detector for the D-Zero upgrade and hardware/software development for the DZero muon detection system.

In the coming years we will focus strongly on data analysis with initial emphasis on the search for the top quark. We are also assuming a major responsibility in the design and construction of a silicon microstrip detector which will be part of the new D0 tracking system for the 1995 luminosity upgrade.

In section 2 we discuss the physics goals and general features of the D-Zero detector. Section 3 describes in detail the activities of the Riverside group.

\section{Physics and detector}

D-Zero is a $4 \pi$ non-magnetic hermetic detector designed for the Tevatron Collider at Fermilab. The evolution of the D-Zero design has benefitted substantially from the experience with the UA1 and UA2 detectors at CERN and the CDF detector at Fermilab. Additional input from the ongoing SSC R\&D program has also proved valuable especially in the areas of readout and tracking designs for the projected Tevatron upgrades. In overall concept D-Zero has been designed to complement the strengths of the existing CDF detector.

The dominant emerging areas for new physics at the Tevarron are the search for the top quark and exploration of the B-physics sector. In addition the general themes of the Tevatron program include: (i) precision measurements of $\mathbf{W}$ and $\mathbf{Z}$ properties (mass differences, decay widths, production mechanisms, rare decays, decay asymmetries, study 
of trilinear boson couplings); (ii) tests of $\mathrm{QCD}$ at very large $\mathrm{Q}^{2}$ (jet topological cross sections, searches for parton compositeness, direct photon studies, searches for quarkgluon phase transitions, measurement of fragmentation functions); (iii) searches for new states which could direct the extension and evolution of the standard model (manifestations of supersymmetry - squarks, gluinos, winos, zinos, sleptons), technicolor particles, heavy leptons, additional gauge bosons, or massive quasistable objects; (iv) measurement of the characteristics of the large cross-section, low $\mathrm{p}_{\mathrm{T}}$ processes (multiplicity distributions, multiparton collisions, emergences of some new phenomena glimpsed in cosmic ray experiments); and (v) sensitivity to qualitatively new phenomena in the heretofore unexplored high energy, large $Q^{2}$ domain.

These physics issues dictated the basic design choices for D-Zero:

i) No central magnetic field is provided. Since the relevant particles to be detected are jets (partons), leptons and non-interacting secondaries (neutrinos, photinos etc.) at large momentum, calorimetric measurement of energies are superior to momentum determination by track curvature. Moreover, a non-magnetic tracking system can be compressed, giving the opportunity for enhanced calorimetry and muon detection.

ii) Lepton identification is of fundamental importance in searching for most high mass states, due to the relative cleanliness of leptonic decay modes of $\mathrm{W}, \mathrm{Z}$ and heavy quarks. Measurement of both electrons and muons over the fullest possible solid angle is highly desirable; electron and muon measurements have quite different systematics so confirmation of new effects in both channels is highly beneficial. Electron channels are superior for precision mass measurements, while muons offer the possibility of seeing a lepton within a jet.

iii) Measurement of the missing transverse energy in an event is crucial for many studies, and achievement of the best possible $\mathrm{E}_{\mathrm{T}}$ resolution is a dominant goal. It is of particular importance to prevent unknown large fluctuations in measured event $\mathrm{E}_{\mathrm{T}}$, particularly far out in the tails of the distribution, in order to avoid having common event 


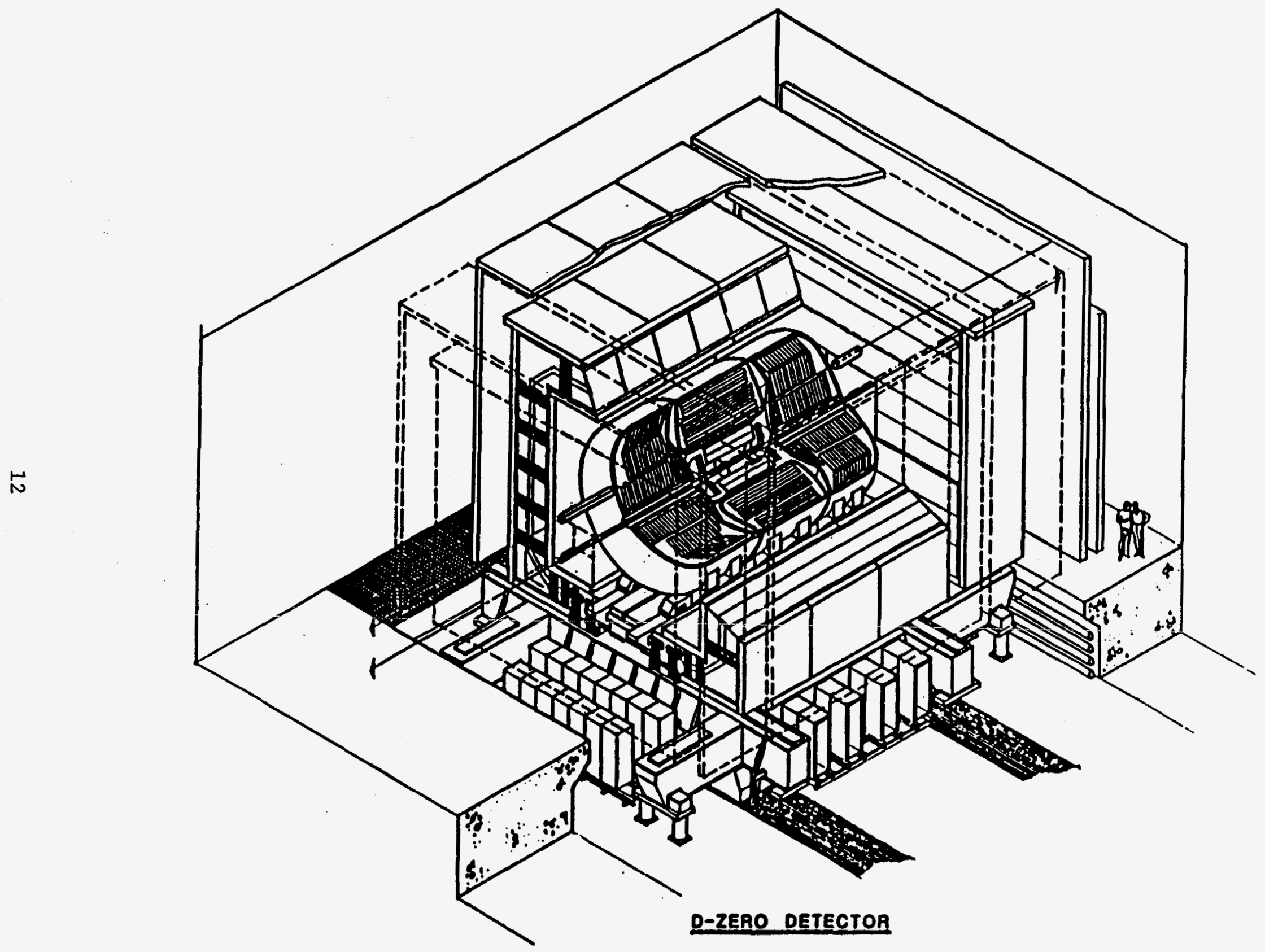

Fig. 1 Schematic View of the DØ Detector 


\section{TABIE 1}

Performance Specifications

\section{Tracking}

Spatial resolution in $r \Delta / \phi$

Spatial resolution in $z$

Two track resolving power ( $\mathrm{r} \phi$ )

Overlapped track rejection by $d E / d x$
$50 \mathrm{\mu m}$ (Vertex chamber) $200 \mu \mathrm{m}$ (Outer chambers)

$10 \mathrm{~mm}$ (Vertex chamber)

$3 \mathrm{~mm}$ (Outer chambers)

$1 \mathrm{~mm}$ (Vertex chamber)

$3 \mathrm{~mm}$ (Outer chambers)

$50: 1$

$50: 1$

$e / \pi$ discrimination

Calorimetry

Energy resolution - electromagnetic - hadronic

Position resolution (EM)

Isolated $e / \pi$ resolving power

Muon Detection

$\delta p / p$ (multiple scattering limit)

$18 \%$

$3 \sigma$ sign determination

\author{
$15 \% / \sqrt{E}(\mathrm{GeV})$ \\ $40 \% / \sqrt{E}(\mathrm{GeV})$ \\ $2 \mathrm{~cm} / \sqrt{E}(\mathrm{GeV})$ \\ $50: 1$
}

\section{Solid Angle coverage}

Drift chambers

$|\eta| \leq 3.5$

Transition radiation

$|\eta| \leq 1$

Calorimetry

$|\eta| \leq 5$

Muon detection

$|\eta| \leq 2.3 \quad(\Delta \Omega=988)$ 
types simulate new physics. Good missing $\mathrm{E}_{\mathrm{T}}$ resolution involves optimization of several features of the detector. Calorimeter coverage should cover the full solid angle with minimal cracks or hot spots. Holes for beam entrance and exit should be limited to $\theta<1^{\circ}$ $(\eta \geq 5)$. Good energy resolution helps control the rms width of the $E_{T}$ distribution; of more importance here is the near equality of response to electrons and hadrons so that fluctuations in hadron shower composition are of minimal importance. Figure 1 is a schematic of the D-Zero detector. Table 1 gives the performance goals for the detector.

The first run with the completed Detector will commence in the Summer of 1991. A substantial fraction of the detector has already been installed in the experimental hall and a Cosmic Ray run is scheduled for the Fall. This will include the central tracking system, the central calorimeters and the barrel muon system and will give a valuable pre-run checkout of a large fraction of the trigger and data-acquisition system.

The remaining pieces of the detector are in the final stages of fabrication and/or are being callibrated in the D-Zero test beam area at Fermilab. These tests should restrict the amount of beam time needed to debug the readout and fine tune the trigger system to the first month or do of the 1991 run. The first data-taking for physics analysis will then follow in the early Fall of 1991.

\section{Riverside Activities}

Our first responsibility upon joining DO was the coordination of the design and construction of the D-Zero high voltage system (section 3.1). This task is almost complete. Concurrently we began a detailed simulation study of a silicon microstrip detector (SVD) for the D-Zero upgrade (section 3.2). This project was completed in 1990 and the final design of an SVD for D0 is now underway. In 1988 we began working on the trigger electronics and off-line reconstruction software for the muon detector (section 3.3). This work is continuing. In the coming year we will initiate software studies for physics analysis (3.4). 


\subsection{High Voltage System Design and Implementation}

At the start of 1987 the Riverside group took over responsibility for the coordination of high voltage system for the D-Zero detector. This included all aspects of both the power system itself and the associated distribution system. Following a three year program of extensive R\&D the design is complete and most of the components have been fabricated in industry. The remaining items are in production and will be shipped to Fermilab over the next few months. The completed high voltage will be the largest and most advanced integrated system of it's kind in operation.

The core of the system is a computer controlled high voltage power supply which has a wide operating range in terms of both output voltage ( 0 to $5.6 \mathrm{kV}+\mathrm{ve}$ and -ve) and current sensitivity ( $32 \mathrm{nA}$ to $1 \mathrm{~mA}$ ). Also built into the design of the supply is fast trip circuitry to shut the supply down in a controlled manner in the event of a detector failure (eg. broken wire), monitoring computer failure, voltage regulation failure or the detection of a large current transient. The supply also has an external shutdown capability which can be interlocked to a safety system, daisy-chained to a neighboring supply (linked channel trip) or triggered by an external software instruction.

The final design is the end-product of work done on 4 generations of prototypes which were designed and tested at Fermilab. The power supply module is a VME based unit which is 3 slots wide and $6 U$ in height. Each unit contains 8 independent channels, giving a channel density of up to 48 channels total per standard J1/J2 Eurosize crate. A module consists of a digital motherboard and 8 mating analog daughterboards. The latter are fixed to either +ve or -ve polarity and operate either as a $1 \mathrm{~mA}, 250 \mathrm{~V}$ to $5.6 \mathrm{kV}$ supply or as a $3 \mathrm{~mA} 0$ to $2 \mathrm{kV}$ supply. Any combination of daughterboards can operate on a single motherboard. Up to a maximum of 4 crates can be interconnected using D-Zero designed interconnect modules, giving a sub-system size of 192 power supply channels. The full DZero design uses 8 such sub-systems. 
The supplies are controlled by two Motorolla 68020 cpus which are resident in a VME controls crate (one per subsystem). These in turn are programmed and monitored by either a local AST 286 PC or remotely (via a tokenring connection) by a VAX station in the main control room. We have designed, written and tested the control software which is resident in both the 68020 's and the PC and this is now fully operational. The Host VAX software is designed and is in the process of implementation. This should be completed by the spring of 1991.

Between the power supplies and the detector is the power distribution system. We designed and prototyped each of the units at Fermilab and they are presently being fabricated at University of Maryland and at New York State University. Fabrication of the units will be completed by the fall of 1990 . The system consists of a passive splitter which is located either in the D-Zero mobile counting house (muon system and calorimeters) or on the detector platform (other detectors). Because of space restrictions we have developed a special 8-conductor cable/connector system which is both compact and very resilient. Both parts were custom designed to suit D-Zero and are now available as commercial products. All of the cabling and connectors have been fabricated, delivered and tested at Fermilab and a large fraction of the cables are now in place in the experimental hall.

The design of the counting room and platform layouts is almost complete and service installation is completed for most of the racks. All that remains to do is a minor revision to add the Inter Cryostat Detector control system as this is now scheduled to be ready for the 1991 run. Our present schedule shows that the full power system will be operational by the Spring of 1991. 


\subsection{Muon detection system}

The D-Zero detector is distinguished by a powerful muon detection system which will be very important in the study of $\mathrm{W}, \mathrm{Z}$ and top physics. As detailed below we are testing and installing electronics for the first level muon trigger and are developing the offline track reconstruction software for the muon system.

\subsubsection{Electronics}

The DO muon detector is constructed in three layers; layer A just before the magnetized iron and two more layers ( $B$ and $C$ ) immediately after the magnetized iron. Figure 2 shows the orientation of the three muon layers with respect to the calorimeter and the magnetized iron. Each layer consists of 4(3) decks for layers A (B and C) respectively. Each deck contains 24 proportional drift tubes (PDT"s). As muons pass through the muon chambers, wire and pad signals are collected by the front end electronics. The pad signal indicating a PDT hit is used for triggering purposes, however, to determine the coordinates of the muons both pad and wire signals are needed.

Figure 3 shows the muon data paths and the different parts which constitute the level 1 trigger. The muon data acquisition system consists of three different types of crates: monitor crates, data crates and trigger crates. Each crate is controlled by a Motorola 68020 microprocessor. The data crates contain a Fanout card, up to 12 Module Address Cards, (MAC's) two ADC;s and a buffer. The trigger crates contain the trigger cards, Octant Trigger Cards (OTC;s), Coarse Centroid Trigger (CCT) cards and a buffer. The MAC uses the pad latch information within a layer, along with a lookup table, to determine if a centroid is present. The centroid is a position marker representing a possible track calculated from a group of pad latches in adjacent decks. The centroid information from the MAC is sent to the CCT where a lookup table is used to make a first level trigger decision and a trigger bit is sent to the trigger processor. Upon receipt of a trigger interrupt, the microprocessor reads the list of PDT hits from the MAC's, and for each hit it digitizes the 
MUON DATA PATHS

Layer C
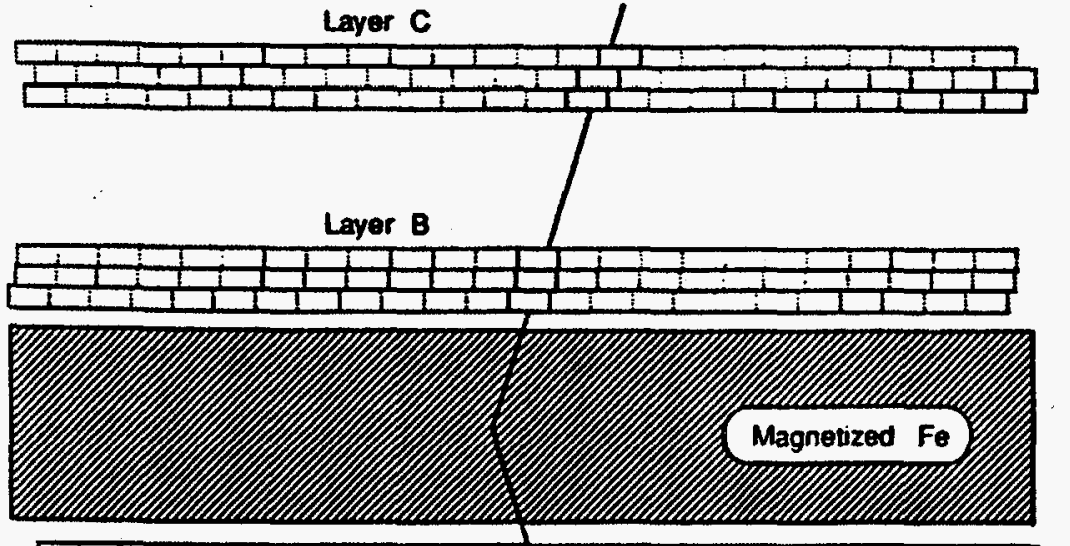

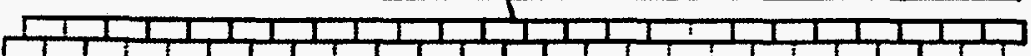
Layer A
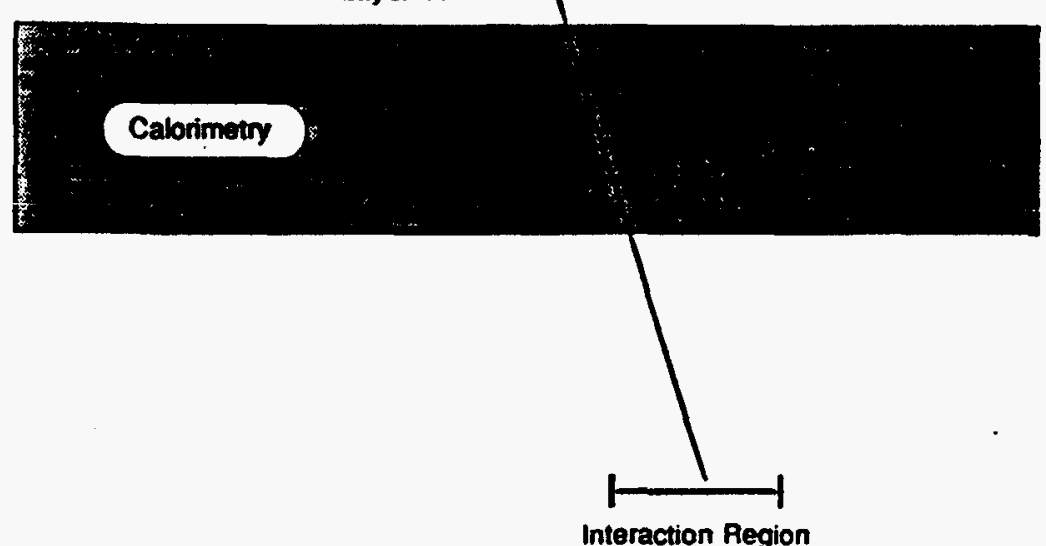

FIG. 2
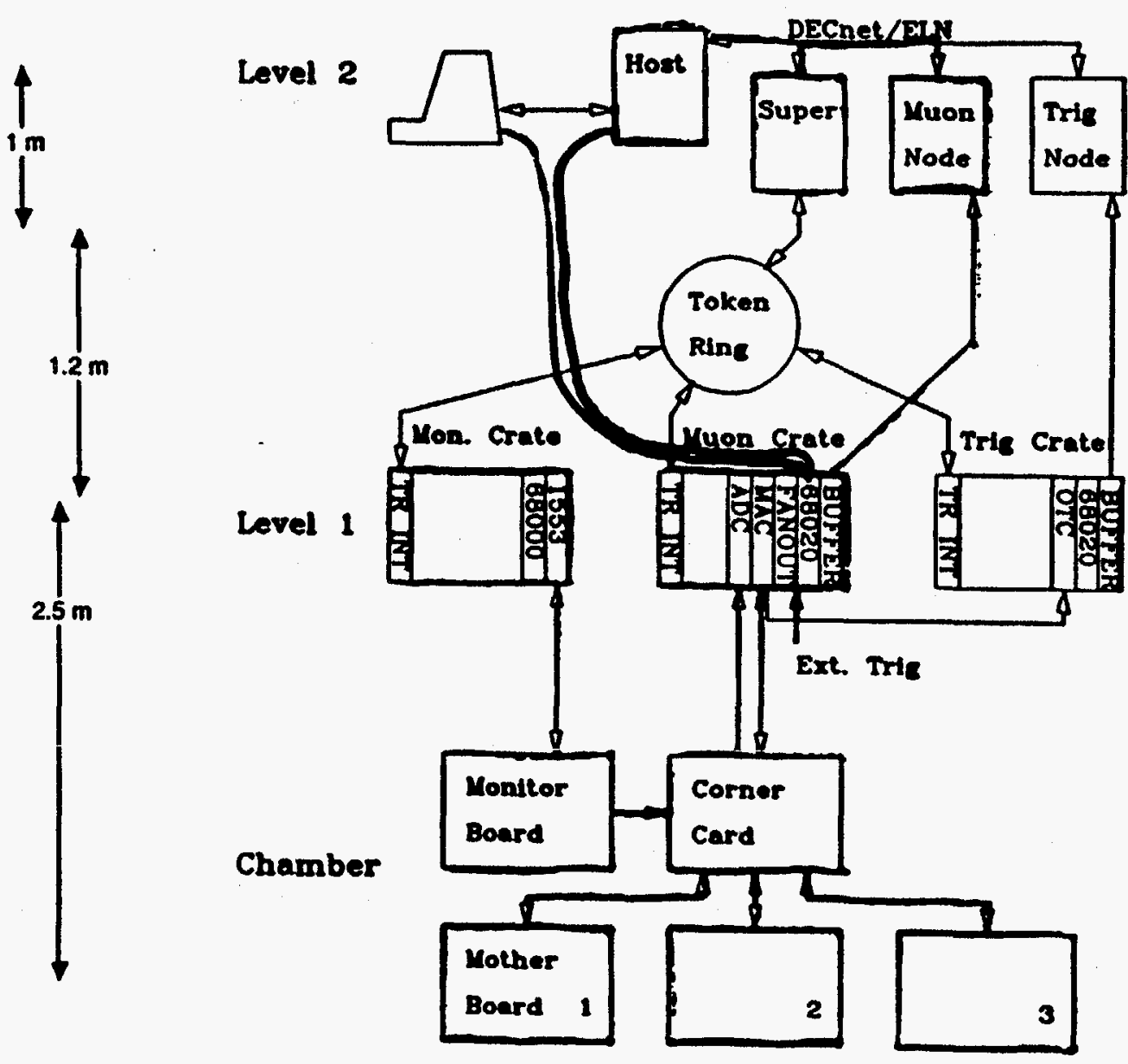

FIG. 3 
wire and pad signals using the ADC's of the data crates. The digitized quantities are then copied to a buffer which in tum sends them to the upper level for data processing.

Our role in the muon level 1 trigger is mainly the development of the MAC design, final processing of the board, testing and debugging. The design development consists of adjusting and updating the board to make it compatible with any new changes in the data acquisition system. The final processing includes modifying the hardware of the board, blasting Programmable Array Logics (PAL's) and stuffing them into the appropriate sockets. The testing procedure includes test software development and use of a test station to diagnose the boards.

All 224 MAC's have been tested, debugged and where necessary repaired, here at UCR with a joint effort of two graduate and four undergraduate students. These MAC's as well as the test station were shipped to Fermilab in July 1990. Currently, a graduate student (Ray Hall), along with an undergraduate student (Tom Fahland) are installing the MAC's and testing them in the acquisition system environment. Hall and postdoctoral researcher M.-J. Yang, both stationed at Fermilab will participate in the installation and commissioning of the muon trigger system through 1991.

\subsubsection{Muon software}

Following our work on the magnetic field maps for the muon toroids we are now developing the off-line program for track reconstruction through the muon detector. The first stage of track reconstruction is to fit the muon trajectory in the toroid with a parabola. This fit will provide initial estimates of the track parameters. A tracking algorithm will then be developed to refit the track taking into account both multiple scattering and energy loss in the calorimeters and toroids. Finally the reconstructed track in the muon detector must be linked to the corresponding track in the inner drift chambers.

The parabola fit has been implemented and tested on a sample of $Z^{\circ} \rightarrow \mu^{+} \mu^{-}$-Monte Carlo events generated with ISAJET and GEANT. This work will continue through 1991 and will correlate with preparations for top and $\mathrm{W}, \mathrm{Z}$ physics. 
A study of the Kalman Technique for the reconstruction of single muon tracks, carried out for the ZEUS experiment in HERA, suggests a greater flexibility and even better results for track parameter estimation than more classical methods. It mau be worthwhile to consider this technique for the D0 experiment too. Future plans are to study aspects of this issue.

\subsection{Silicon micro-vertex detector}

For the luminosity of $5 \times 10^{31} \mathrm{~cm}^{-2} \mathrm{~s}^{-1}$ anticipated in the 1995 Tevatron collider run it will be necessry to totally replace the present D0 drift/TRD tracking system. It is likely that one element of the upgraded tracking system will be a silicon microstrip vertex detector (SVD). S. Wimpenny has recently been appointed co-chair of a D0 working group which will investigate the design and physics potential of a silicon microstrip detector for the $\mathrm{D} 0$ experiment.

The UCR group will play a major role in R \& D and prototyping for the SVD, using the on-campus facility developed for this purpose. As part of the planning for the DO upgrade UCR has recently completed a detailed simulation study of the capabilities of a central barrel-type SVD in a 'non- magnetic" environment such as D0 in which hadron momenta are unmeasured [1,2]. We conclude that such a device can eficiently tag b-and cquark jets in $\mathrm{D} 0$, and would be important in identifying the top quark.

\subsubsection{Simulation studies}

The silicon microvertex detector in this study consists of four radial layers of silicon microstrips surrounding the beam pipe. The strips run parallel to the beam pipe. The SVD had a position resolution of $10 \mu \mathrm{m}[3]$ and rapidity acceptance $|\eta|<2.7$, and provides a two-dimensional track projection in the plane transverse to the colliding beams. The GEANT program was used to track particles through the SVD, and track finding and fitting were fully simulated. 
The ISAJET Monte Carlo program was used to generate $Q C D$ bБ and gluon-gluon production in equal amounts with $\mathrm{pT}$ of $20 \mathrm{GeV} / \mathrm{c}$ at $\sqrt{ } \mathrm{s}$ of $1.8 \mathrm{TeV}$. Two parameters were investigated for distinguishing $b$-jets from gluon jets: these were (i) the mean (projected) impact parameter $\langle d\rangle$ of tracks within a jet, and (ii), the (projected) distance $L$ from the primary vertex to a secondary vertex within the jet. These parameters were applied to the inner core of jets $(\Delta R<0.4)$ to minimize the problem of low momentum tracks faking large impact parameters. The effectiveness of the parameter $L / \sigma_{L}$ ( where $\sigma_{L}$ is the error on $L$ ) in separating b-quark jets from gluon jets is illustrated in fig. 4, which shows the distributions of $L / \sigma_{L}$ for both b-quark jets and gluon jets. The effects of various cuts on $L / \sigma_{L}$ are summarized in Table 2 . The condition $L / \sigma_{L}>3$ was found to enhance the ratio bb : gluongluon events by a factor of 28 while retaining $25 \%$ of bБ events.

A similar comparison was made for an equal number of $b \bar{b}$ and and $c \bar{c}$ pairs at $\mathrm{pT}$ of $20 \mathrm{GeV} / \mathrm{c}$ (Table 2). As expected b-quarks are not as readily separated from c-quarks as from gluons. Thus the condition $L / \sigma_{L}>3$ only enhances the $b \bar{b}: c \bar{c}$ ratio by a factor of 3.8 .

For high momentum tracks ( $\mathrm{pT}>4 \mathrm{GeV} / \mathrm{c}$ ) our study gave an error $\Delta \mathrm{d} \approx 30 \mu \mathrm{m}$ on the (projected) track impact parameter for tracks traversing four planes of the SVD. The fraction of such tracks from B-hadron decay having $d / \sigma_{d}>3$ is $37 \%$ (see fig. 4). Since electron and muon momenta are measured in the D0 detector it should be possible to identify a substantial fraction of semi-leptonic B-decays in DO through lepton impact parameter measurements.

These results were applied to a study of mass $120 \mathrm{GeV} / \mathrm{c}^{2}$ top quark production at $\sqrt{s}$ of $1.8 \mathrm{TeV}$. The efficiency of the $\mathrm{L} / \sigma_{\mathrm{L}}$ parameter in identifying the "isolated lepton + jets" topology of $t \bar{t}$ in the presence of "W + jets" background was investigated. The $b-$ tagging algorithm $L / \sigma_{L}>3$, improved the signal-to-background ratio by a factor of 10 . 


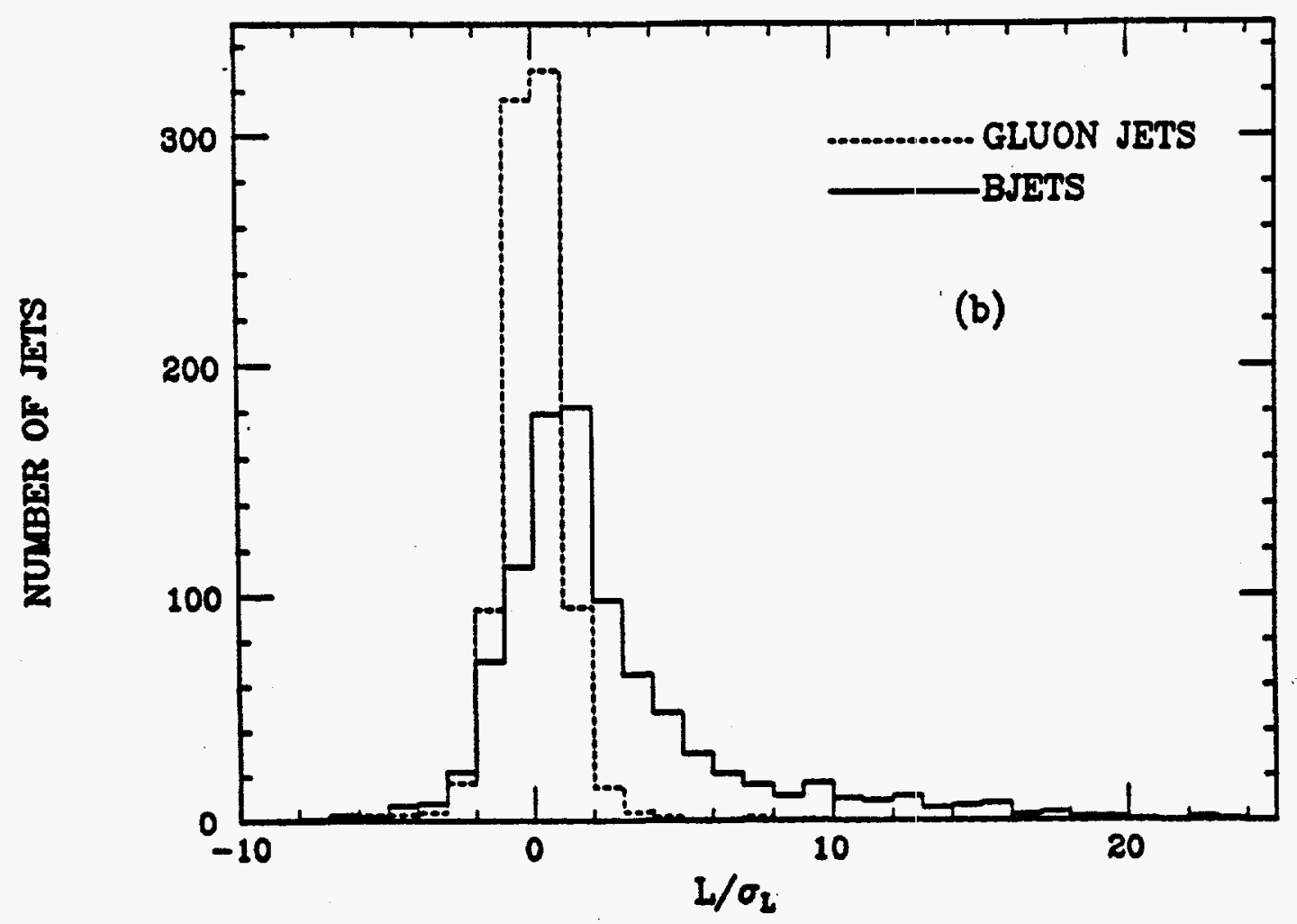

FIG. 4

\section{TABLE 2}

\begin{tabular}{ccccccc}
$\frac{L_{L}}{\sigma_{L}} \min$ & $\# b$ jets & \#c-jets & \# gluon jets & brg ratio & blc ratio & $\begin{array}{c}\text { Efficiency } \\
(\%)\end{array}$ \\
\hline 1 & 555 & 276 & 120 & $4.6 \pm 0.5$ & $2.0 \pm 0.1$ & $49.9 \pm 2.1$ \\
2 & 373 & 120 & 25 & $14.9 \pm 3.1$ & $3.1 \pm 0.3$ & $33.5 \pm 1.7$ \\
3 & 275 & 73 & 10 & $27.5 \pm 8.9$ & $3.8 \pm 0.5$ & $24.7 \pm 1.5$ \\
4 & 210 & 45 & 6 & $35.0 \pm 14.5$ & $4.7 \pm 0.8$ & $18.9 \pm 1.3$ \\
5 & 162 & 32 & 4 & $40.5 \pm 20.5$ & $5.1 \pm 1.0$ & $14.6 \pm 1.1$
\end{tabular}




\subsubsection{Hardware studies}

We propose to investigate aspects of the silicon detectors and readout electronics required to construct the detector. As well as being essential for the $\mathrm{D} 0$ silicon detector, we believe that such a program will be useful for progressing towards more advanced silicon detector systems such as those being proposed for the SSC [4]. Further details are given in Section 1c, Detector Development.

\section{References}

1.) D. Smith, "Identification of b-jets in D-Zero with a Barrel Silicon Vertex Detector", D-Zero Note no. 830, (1989).

2.) J. Ellison, A. Kernan and D. Smith, "Simulation of a Silicon Vertex Detector in the D-Zero Experiment", D0 Note 975, to be submitted to Nucl. Instr. and Meth.

3.) D. Joyce et al., "Silicon Detector Tests with the RAL Microplex Readout Chip", proceedings of the International Conference on Advanced Technology and Particle Physics, Como, Italy 13th-17th June 1988, Nucl. Instr. and Meth. A279 (1989) 289.

4.) J. Ellison, S. Wimpenny et al., "SSC Detector Subsystem Proposal: Subsystem R\&D Proposal to Develop a Silicon Tracking System", 1989.

\subsection{Physics Analysis}

With D0 turn-on less than a year away we are shifting our activities from hardware to physics analysis. The primary objective for $\mathrm{D} 0$ is detection and study of the top quark. CDF has established that the top quark mass exceeds $89 \mathrm{GeV} / \mathrm{c}^{2}$, while theoretical considerations set an upper bound around $200 \mathrm{GeV} / \mathrm{c}^{2}$. Thus top will decay to a real W:

$$
\begin{array}{rl}
\mathrm{t} \rightarrow \mathrm{W}+\mathrm{b} & \mathrm{I} \\
& \rightarrow l \mathrm{v}_{\mathrm{e}} \\
& \rightarrow \mathrm{q} \overline{\mathrm{q}}
\end{array}
$$


and $\mathfrak{t}$ ) final states will be characterised by multiple leptons and/or jets. DO is an ideal detector for heavy quark studies since it is hermetic with precise and uniform muon, electron and jet capability covering almost $4 \pi$ in solid angle.

In the next few years we will devote at least $50 \%$ of our effort to physics analysis. In the immediate future we will concentrate on triggering strategies for top and on backgrounds to top. These studies will require high statistics Monte Carlo event simulations. We also plan to study the physics of the $\mathrm{W}, \mathrm{Z}$ sector in which we have a continuing interest from the UAl experiment. Elucidation of high $\mathrm{p}_{\mathrm{T}} \mathrm{W}$ production, apart from the intrinsic interest, forms the major background to the top quark. We outline below a program for $\mathrm{W}, \mathrm{Z}$ studies. We are particularly interested in the physics topics c,d,e,g in Phase 2.

As is well known Monte Carlo simulations play an essential role in extracting physics measurements from collider data. A variety of Monte Carlo programs -- ISAJET, PYTHIA, PAPAGENO - are available for simulating high energy hadronic interactions. It will be important to compare these programs and to evaluate their accuracy for different processes.

\section{W.Z Physics}

\section{Phase 1 - getting started}

1) Set up basic analysis structure for $W$ and $Z$ event slection.

This needs to include the following:

- raw data/event processing interface

- Monte Carlo event processing interface

- suite of cut routines

- $\pi / \mathbf{K}$ decays

- misidentified $h-\mu$ matches

- software trigger validation (events will not necessarily satisfy the 
"W/Z" hardware trigger)

- electron mis-identification near jets

- suite of utility routines:

- isolation

- track quality parameters

- physics variables

- W/Z kinematics routines

- Monte Cario event fluctuation code to study effects of experimental resolution in chosen variables.

2) Mini/Micro DST writing code

Phase 2 - Physics

a) Electroweak

- Set up mass fitting code for W mass (electron,muon).

- Set up mass fitting code for $\mathrm{Z}^{\circ}$ mass (electron, muon).

- Write interface for $1+2$ inside basic analysis structure.

- Study experimental resolutions + background using data + GEANT.

- Write a fast mini - Monte Carlo for resolution studies.

- Get best value of $\mathrm{W}$ mass and $\mathrm{W}-\mathrm{Z}$ mass difference.

- Fix energy scale using $Z$ mass from LEP.

- Compute Standard Model parameters and study error correlations.

b) Production + Decay Properties

- Interface EKS generator into GEANT.

- Compare EKS/GEANT with data results for $x_{W}, x_{z}, p_{T}{ }_{T}, p_{T}{ }_{T}$ jet multiplicities.

- Study effects of changing experimental resolution.

- Study effects of using different parton distributions in EKS generator.

- Compare $\mu, e, \tau$ cross-sections $\rightarrow$ universality. 
c) $Q C D+$ Top Background

- Use EKS/GEANT to study $Z(W)+$ multijet topologies. Pay special

attention to jet thresholds and jet-find efficiencies.

- Look for 'non-standard' effects - e.g. $W^{+} W^{-}$pairs.

d) W Decay Width

- use ratios of $\sigma \mathrm{B}_{\mathrm{W}} / \sigma \cdot \mathrm{B}_{\mathrm{Z}}$, take $\mathrm{Z}$ width from LEP and invert the calculation which was originally used to set limits on $\mathrm{N}_{v}$ through $\Gamma_{\mathrm{z}}$.

e)Asymmetrics

- study $\operatorname{Sin}^{2} \theta_{W}$ dependence of $Z^{\circ} \rightarrow l l$ decay angular distribution.

- study the $\mathrm{W}^{+} \mathrm{W}^{-}$decay asymmetry as a function of rapidity and investigate how sensitive this is to the parton distribution functions $(\eta \geq 1.5)$.

f) Non-standard decays

- survey possible non-standard $\mathrm{W}, \mathrm{Z}$ decay modes

- look for signatures in data.

g) Addtional IVB's

- search for heavy $W^{\prime}$ or $Z$ ' decays which decay via standard $W$ and $Z$ into final state particles. These are "expected" to have masses $\approx 200-300 \mathrm{GeV}$.

h) Zoo Events

Search data for anything "abnormal".

i) Radiative Decays

- compute QED bremsstrahlung corrections for $\mu$ decays.

- study data to find rates and thresholds for identifiable radiative $\mathrm{W}$ and $\mathrm{Z}$ decays.

- compare real with predicted rates and kinematic dependences. 
We have set up a laboratory at Riverside devoted to development and evaluation of high resolution silicon tracking devices for use in the hadron collider environment. This lab, which was set up using S. Wimpenny's initial complement from UCR, enables us to perform extensive evaluation and R\&D on Silicon detectors. We plan a continual expansion of this lab in the coming years. As members of the D-Zero and SDC Collaborations at the Tevatron and SSC, respectively, we are pursuing studies of double sided silicon strip detectors and their readout with a view to building devices for the inner tracking systems of these detectors.

\section{D-ZERO}

One element of the upgrade of the D-Zero detector is the construction of a silicon vertex detector for fast tracking and vertex reconstruction. This forms the inner part of the redesigned D-Zero tracking system and is intended to be used both in track reconstruction and to provide a fast $\mathrm{z}$-coordinate measurement for the Level 2 trigger. The $\mathrm{z}$-coordinate could be obtained using double-sided microstrips with stereo readout, although other methods may be possible such as 'short strips' or pixel devices. The UCR group will play a major role in the design and testing of the detector.

Using our facilities on campus we will concentrate on evaluation of different designs of double sided silicon detectors. This will include the full evaluation of double sided microstrip detectors with capacitive coupling.

For D-Zero the radiation levels forseen for the 1993 and 1995 runs are not a problem for the strip detectors but they are problematic for current VLSI readout electronics. For example, for the 1995 Tevatron run we expect a charged particle dose of $600 \mathrm{Krad} / \mathrm{r}_{\perp}^{2} \mathrm{~cm}^{-2}$ where $r_{\perp}(\mathrm{cm})$ is the perpendicular distance from the beam line. This necessitates the development of new radiation hard readout electronics. Further, since the 
time between bunch crossings will ultimately be $400 \mathrm{~ns}$ at the upgraded Tevatron, we need to develop a readout chip which can handle such a rate. Although we do not plan to be directly involved in the chip design work, we will be involved in the subsequent chip evaluation and will set up a test bench for chip evaluation at UCR. Work on chip development is presently in progress for the CDF Collaboration who are developing a radiation hard version of the Berkeley SVX chip. It is unclear whether this chip will be suitable for D-Zero use and further development work may be needed to meet the D-Zero design specifications.

Finally, we anticipate that a significant amount of work will be required to design the mechanical support systems for the SVD. A layer to layer hardware alignment of less than $25 \mu \mathrm{m}$ in $\mathrm{r} \phi$ must be achieved, and cooling of the heat dissipated by the electronics must be provided to avoid stress and movement of the silicon. Also, we will review suitable low radiation length materials such as beryllium, carbon fiber, boron carbide, rohacell, carbon fiber, boron carbide to be used for the mechanical support.

\section{SSC}

The design of the SDC Collaboration detector for the SSC[2] calls for a very large silicon tracking system which will form the basis of the charged particle tracking at radii between 15 and $60 \mathrm{~cm}$ from the beam (Fig.5). This covers an area of about $32 \mathrm{~m}^{2}$ and the system will contain about 10 million readout channels. The design represents a significant step beyond any of the present generation of vertex detectors proposed for hadron colliders.

The tracker consists of $300 \mu \mathrm{m}$ thick double sided detectors with axial strips on one side and stereo strips on the other side. Pairs of such detectors with opposite stereo pitch are arranged in superlayers in a cylindrical barrel section and also in planar endcaps. The superlayers allow local hit association into segments that are then further associated into tracks. The transition from barrel to intermediate angle geometry is near 45 degrees to 


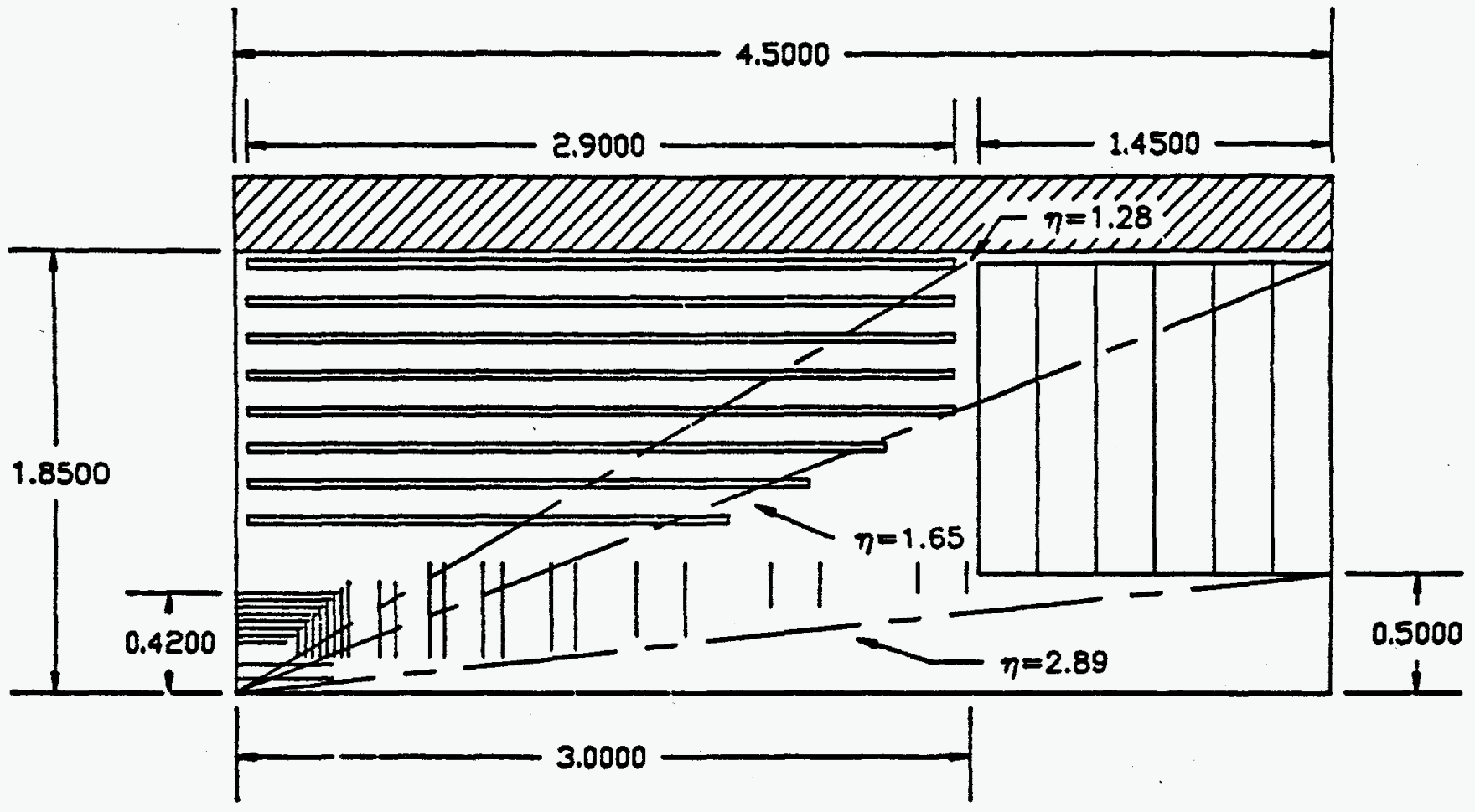

A pixel, silicon strip, and wire chamber tracking system for the SDC detector.

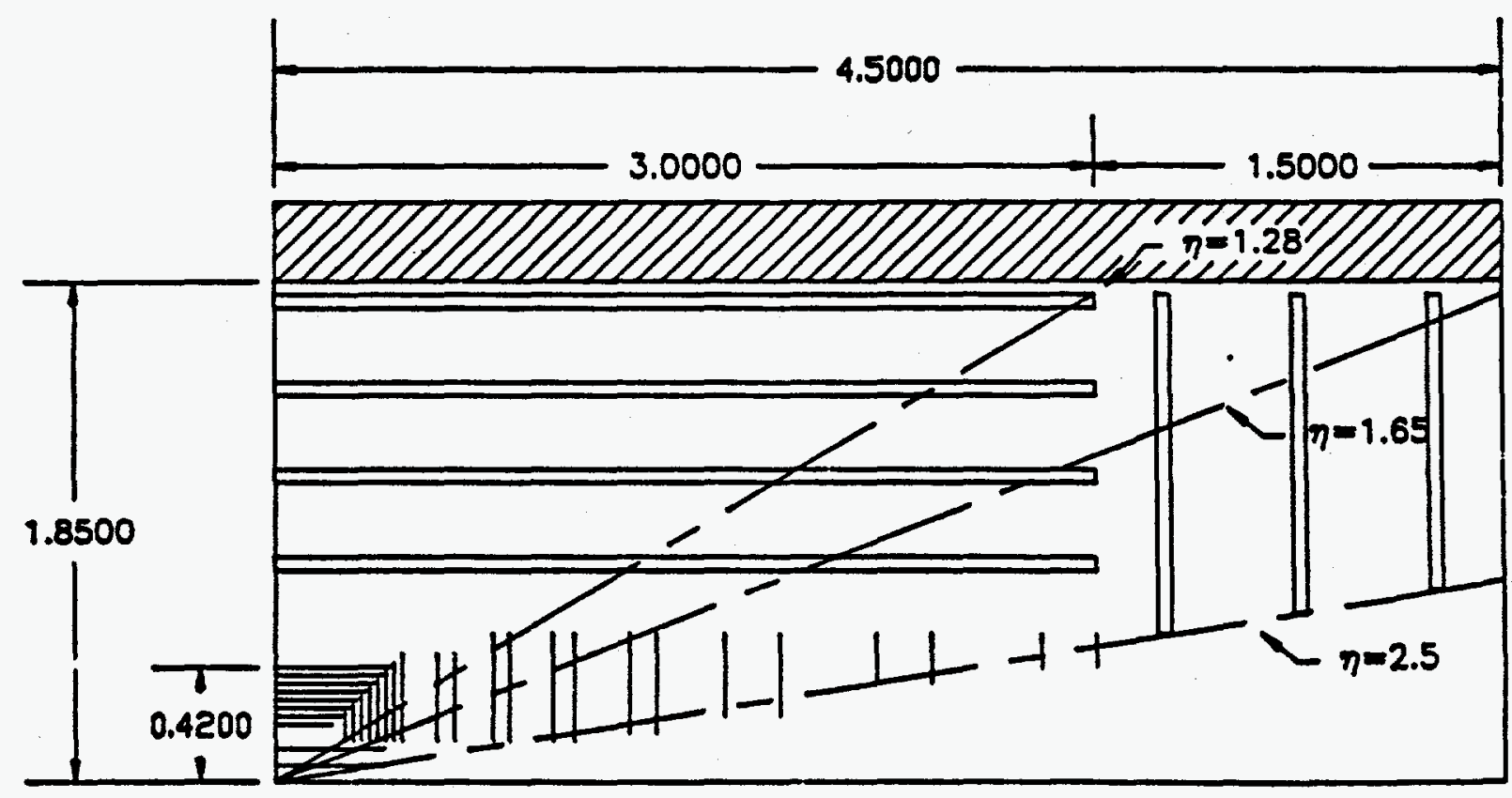

A pixel, silicon strip, and scintillating fiber tracking system for the SDC detector.

FIG. 5 
minimize the thickness of material traversed by particles. The intermediate angle detectors are spaced along the beam at a distance of nearly $3 \mathrm{~m}$ from the interaction point to allow good momentum measurement at large rapidities.

Complimentary to our silicon microvertex detector project for D-Zero we are participating in the SSC Silicon Tracking subsytem R\&D work [1]. The last collaboration meeting for this group was hosted by J. Ellison at UC Riverside on 15/16 July 1990 . Although many of the areas of study are common to both projects, the SSC poses additional problems due to the very high rates, the harsh radiation environment and the very large area of silicon required for the proposed tracking system. We will concentrate our efforts in the following areas :

(a) Double-sided microstrip detectors with stereo strips for the measurement of the $z$-coordinate. Of particular interest is the overall performance of double-sided detectors (noise, efficiency, radiation hardness) and the resolution for the $z$ measurement.

(b) Radiation hardness of double-sided silicon detectors and their associated readout electronics. This is of special importance at the SSC interaction regions due to the high radiation levels expected.

(c) Test beam evaluation of prototype modules consisting of double-sided microstrip detectors and readout electronics. We will also evaluate modules which have been previously irradiated with doses simulating the expected exposure at the SSC.

(d) Aspects of mechanical support structures and cooling for silicon detector systems. The experience which we will gain from the D-Zero SVD will be carried over to the larger scale problem at the SSC.

(e) Monte Carlo studies of the performance of a tracking system incorporating silicon detectors. This will determine the overall performance of the silicon tracking system. Pattern recognition, momentum resolution, vertex reconstruction 
and the efficiency for linking of tracks to the outer detectors will be studied to allow optimization of the detector.

\section{References}

1.) J. Ellison, S. Wimpenny et al., "SSC Detector Subsystem Proposal: Subsytem R\&D Proposal to Develop a Silicon Tracking System, 1989.

2.) J. Ellison, S. Wimpenny et al., "Solenoidal Detector Collaboration Expression of Interest to Construct and Operate a Detector at the Superconducting Super Collider", May 24th 1990. 
$1 d$. NUCLEON STRUCTURE FUNCTIONS

At the heart of all calculations of hard scattering cross-sections are parameterizations of the parton distributions functions $q\left(x, Q^{2}\right), q\left(x, Q^{2}\right)$ and $g\left(x, Q^{2}\right)$. These, in turn, are derived from measurements of the Deep Inelastic Structure Functions $F_{1}\left(x, Q^{2}\right)$, $F_{2}\left(x, Q^{2}\right)$, and $F_{3}\left(x, Q^{2}\right)$ and it is on the reliability of these measurements on which the parton distribution functions depend.

In general the experimental data are in quite good agreement with the exception of the region of small Bjorken $x(x \leq 0.2)$ where significant discrepancies exist. This is particularly unfortunate as this is the most important kinematic region for calculations pertaining to cross-sections at the Tevatron and SSC.

As a part of an ongoing study of the deep inelastic data we are investigating two of the principal experimental datasets - those of the EMC and BCDMS Muon Collaborations at CERN. It is the inconsistencies between these two sets of data which give rise to some of the largest uncertainties in the parton distributions.

The EMC and BCDMS have published high precision measurements on the structure function $F_{2}\left(x, Q^{2}\right)$ measured using hydrogen [1,2], carbon [3] and iron targets [4]. Here we consider only the hydrogen data. The same basic problems are also present in the other datasets. The raw datasets are compared in Fig. 6 from which it can be seen that, in terms of their broad characteristics, the data agree. The $Q^{2}$-dependence of $F_{2}(x)$ is similar for both experiments but when fitted results in significantly different results for $\Lambda$ (Table 3). The $\mathbf{x}$-dependence, however is significantly different and inconsistent with a simple normalization difference between the two experiments. This is inconsistent with the statistical and systematic errors quoted by the two groups.

We have studied the analysis methods used by the two groups in detail and find that there are several differences in both the theoretical input and methods used to extract $F_{2}$. Careful studies of the treatment of the electromagnetic radiative corrections [5] and the 
calculations of the ratio $R=\sigma_{L} / \sigma_{T}[6]$ suggest that these may be responsible for some of the differences between the two sets of data.

To further investigate this requires the re-analysis of the two sets of data using the same assumptions. We have almost completed a re-analysis of the older EMC experiment and have begun to study the BCDMS data. Preliminary results suggest that the differences are decreased and that the $Q^{2}$-dependences of $F_{2}$, and hence the value of $\Lambda$, are now in very close agreement (see Table 3 and Figs. 7,8). The $x$-dependences still differ but not by as much as before and the difference is now consistent with a normalization difference of around $5 \%$ for the $x$-range $x \leq 0.4$ which is within the normalization errors of the two datasets (cf. this was $10 \%$ in the published data). The differences at large values of $\mathrm{x}$ are more difficult to interpret but may just be the result of statistical fluctuations within the EMC data. Further work is necessary to finalize these studies. This is in progress.

In addition to the BCDMS muon data we also have access to the electron-proton data from SLAC. The data from all of the SLAC experiments has recently been reanalyzed, using assumptions similar to those in our EMC-BCDMS study, by the E139/E140 [7,8]. While there is little kinematical overlap with the two CERN groups the data provide an independent measure of the $x$-dependence of $F_{2}$. Two versions of these data; one using the results direct from the SLAC analysis (open squares) and the second using results re-extracted using our treatment of R [ ] (solid points) are compared to our results in Fig.9. The agreement between the electron and muon datasets is good but further work is needed to see if the SLAC data is in better agreement with either set of muon data.

We plan to continue this work with the aim of resolving some of the discrepancies in the data and producing a better set of parton distribution parameterizations for use in QCD calculations. 
FIG. 6
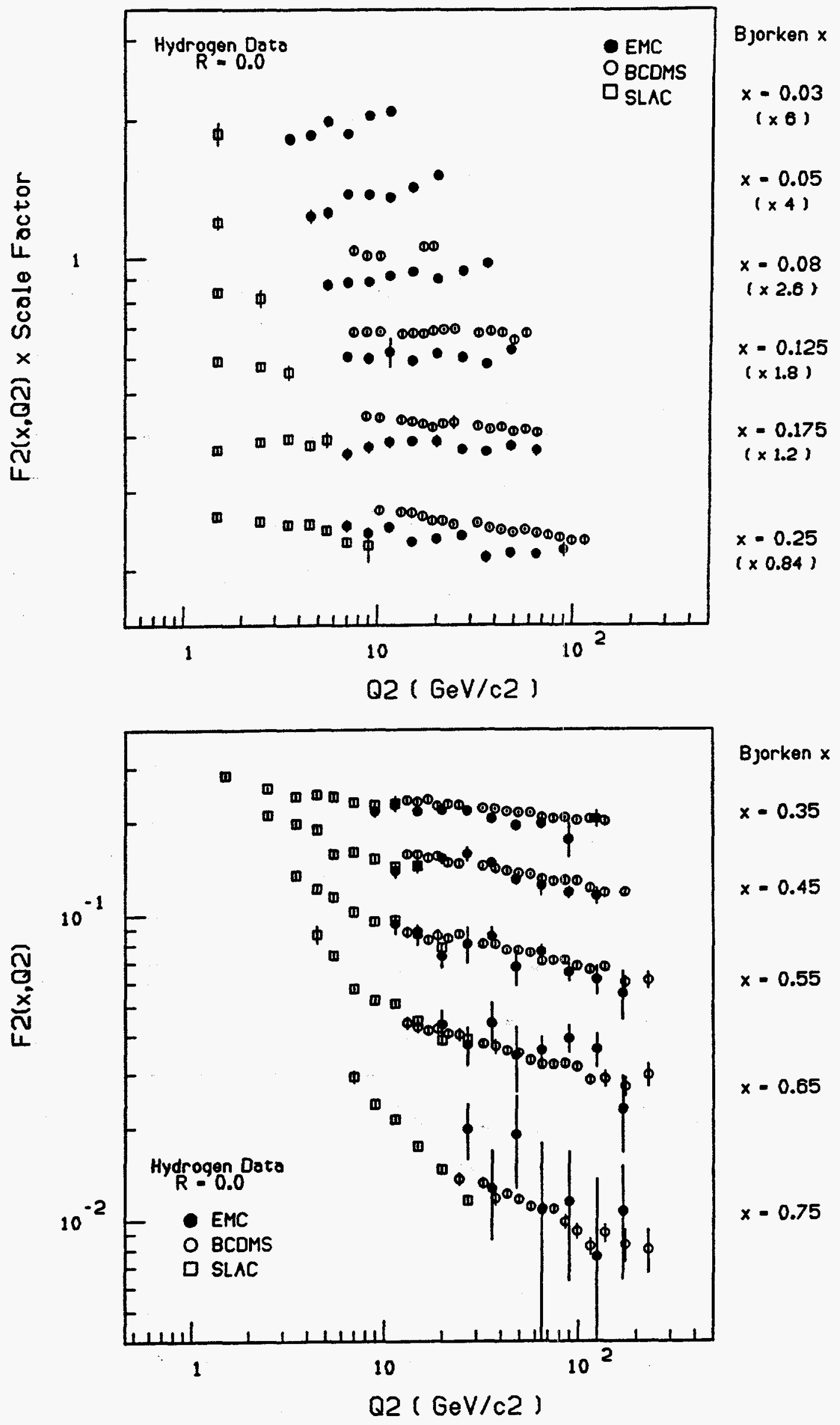
FIG. 7

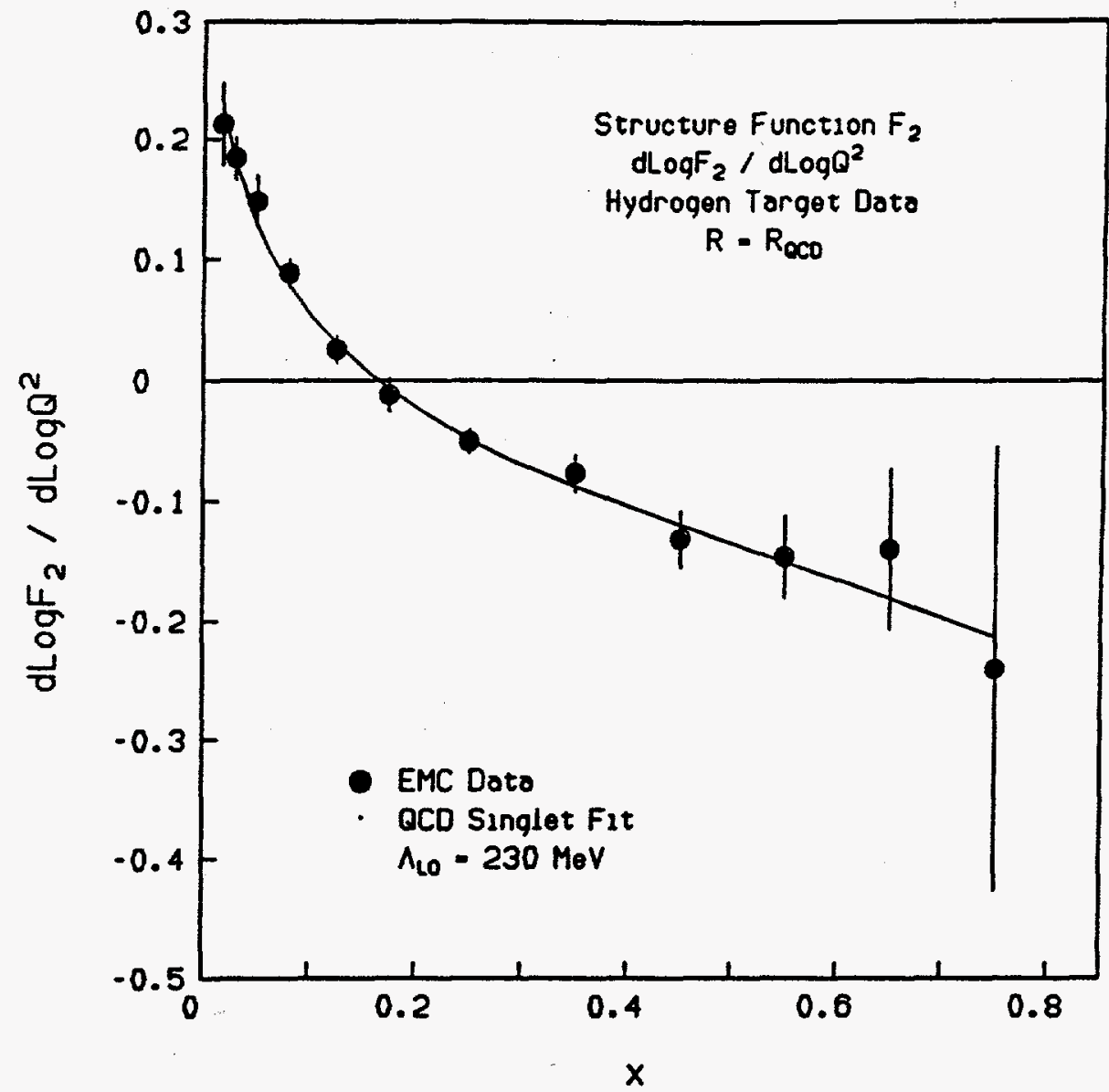

FIG. 8

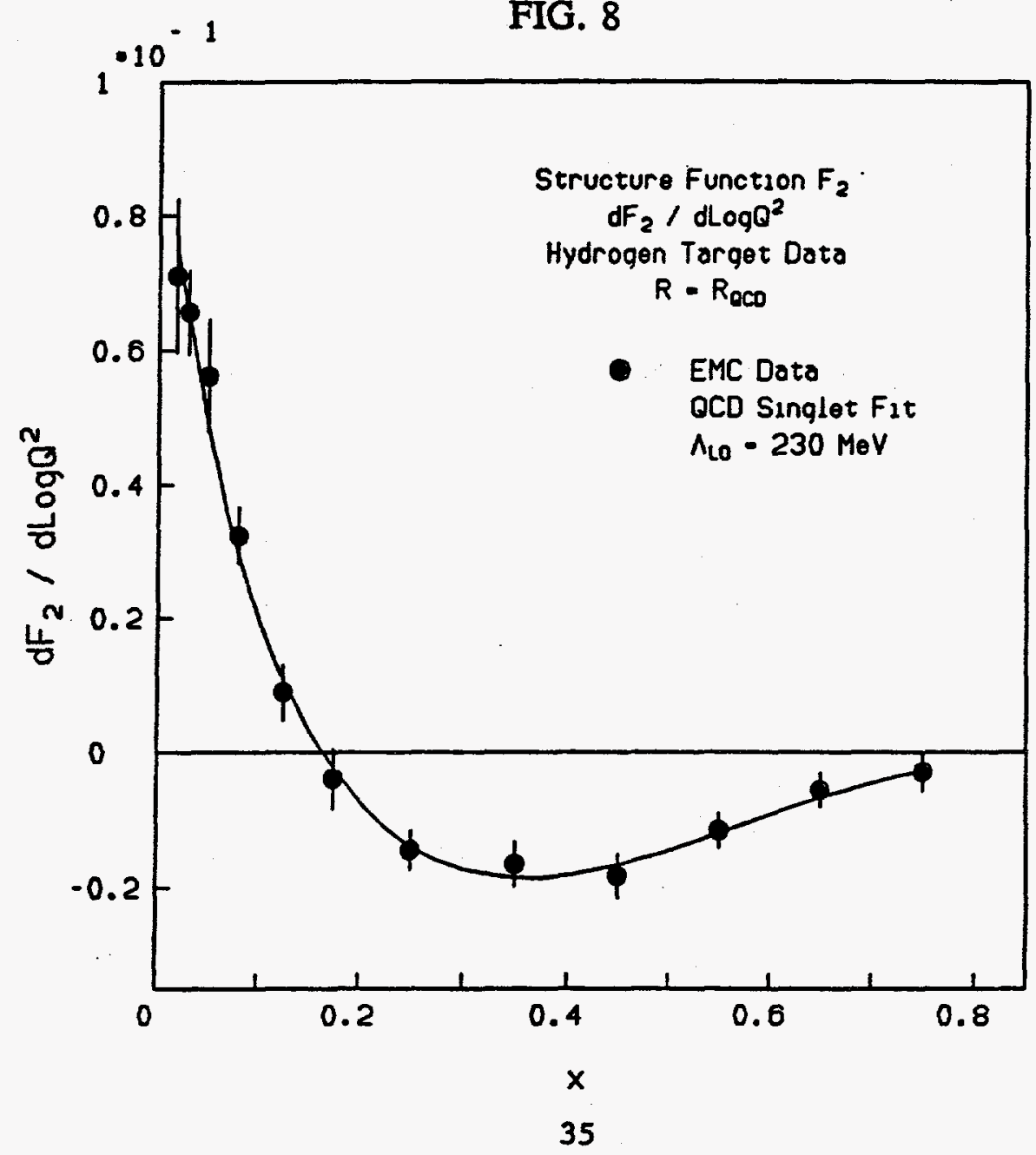


FIG. 9
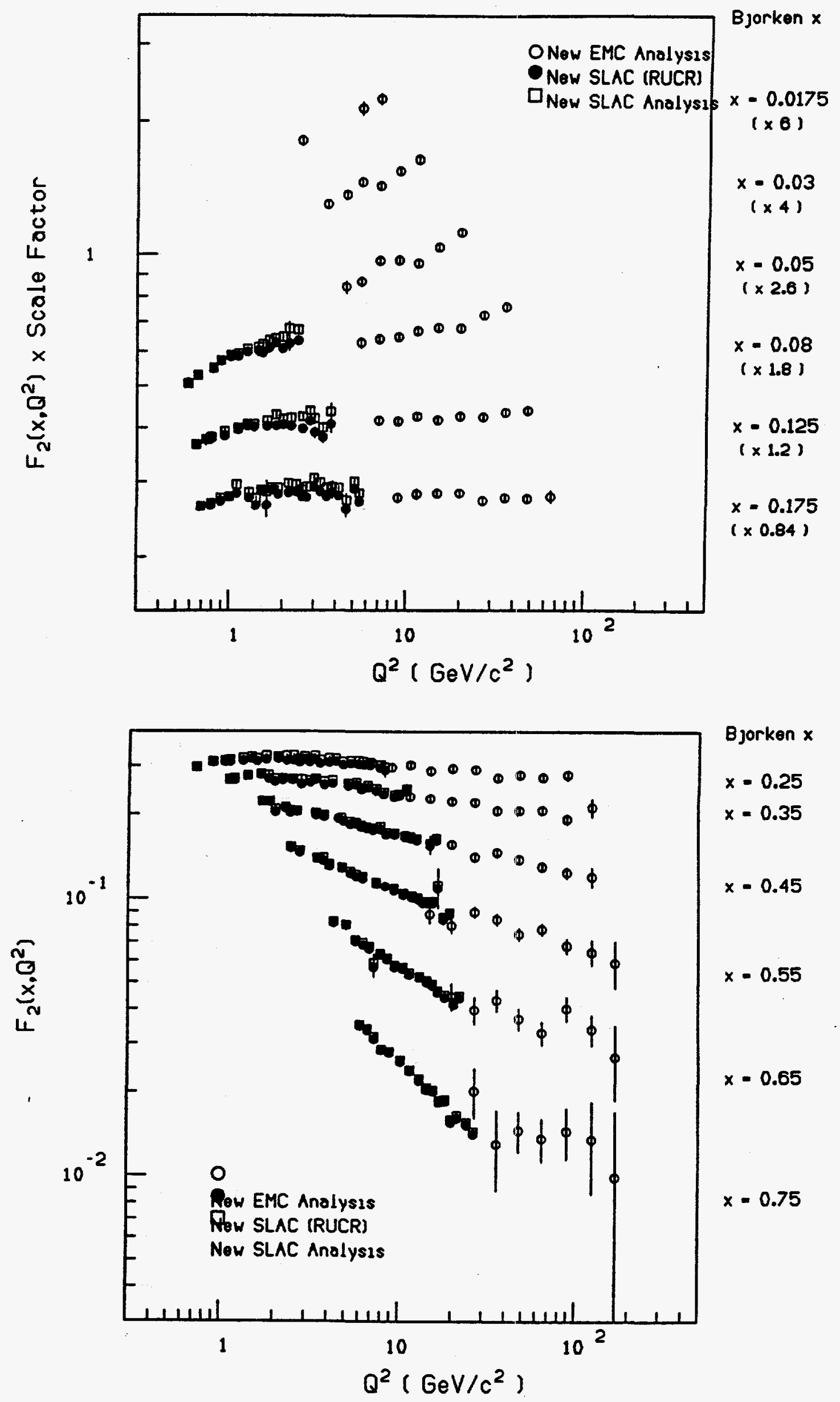


\begin{tabular}{|cccc|}
\hline \multicolumn{3}{c|}{ TABLE 3 } \\
QCD Fit Results \\
QCD Fit & $\begin{array}{c}\text { This Analysis } \\
\text { (preliminary) } \\
\text { Leading Order }\end{array}$ & EMC[1] & BCDMS[2] \\
Singlet & $228 \pm 80 \mathrm{MeV}$ & $90 \mathrm{MeV}$ & $215 \pm 27 \mathrm{MeV}$ \\
$\begin{array}{c}\text { Next to Leading } \\
\text { Order } \\
\text { Non-Singlet }\end{array}$ & $209 \pm 80 \mathrm{MeV}$ & $105 \pm 101 \mathrm{MeV}$ & $224 \pm 21 \mathrm{MeV}$ \\
\hline
\end{tabular}




\section{References}

1.) J.J. Aubert et al., Nucl. Phys. B259, 189, (1985).

2.) A.C. Benvenutti et al., CERN-EP/89-06, (1989).

3.) A.C. Benvenutti et al., Phys. Lett. 195B, 91, (1987).

4.) J.J. Aubert et al., Nucl. Phys. B272, 158, (1987).

5.) C. Lietzke, S.J. Wimpenny, "A Comparison of the RadEMC and Bardin-Shumeiko Radiative Correction Programs", U.C. Riverside Report No. UCRDIS-89-06, (1989).

6.) K. Bazizi, S.J.Wimpenny, "A Study of the Properties of the Ratio $R=\sigma_{L} / \sigma_{T}$ ", U.C. Riverside Report No. UCRDIS-90-04.

7.) S. Dasu et al.(E139/E140 Collaboration), paper contributed to the International Europhysics Conference on High Energy Physics, Madrid, September 1989, University of Rochester Preprint No. UR-1119, 1989.

8.) S. Rock, L.W. Whitlow, private communications. 
$e^{+} e^{-}$Collider Physics

and

Neutrino Physics 


\subsection{Task A2}

\subsubsection{Program}

The physics program of Task A2 has been the systematic study of leptons and hadrons at $e^{+} e^{-}$colliders. In the late 70s and early 80s, the physics analyses of the TPC $/ 2 \gamma$ experiment at PEP were focussed on $e^{+} e^{-}$annihilation processes and twophoton interaction processes. Since 1984 the major effort has been the construction of the OPAL detector at LEP and, since last fall, its exploitation for physics. Results from data taken during the LEP run of 1989 and during this year's run cover a broad spectrum of physics, ranging from precision measurement of electroweak parameters to the determination of the running coupling constant of the strong interaction. A small fraction of our group is participating in an ongoing neutrino oscillation program at Los Alamos. The experiment LSND is expected to take data beginning in 1992. Our program during the next five years will be the continuation of the physics exploitation of LEP and LEP2, and the design and construction of a detector for an experiment at a future high energy collider. 


\subsubsection{Personnel}

Ph.D. Physicists

M. Dittmar

W. Gorn

J.G. Layter

J.K. Riles

B.C. Shen

G.J. VanDalen

To be named

\section{Graduate Students}

P.P. Altice

E.G. Heflin

C. Ho

T. Huen

W.J. Larson

J. Letts

H. Oh

B. O'Neill

A. Smith

W. Strossman

Visiting Scientist

Y.H. Yang
Postgraduate Researcher

Staff Research Associate

Adjunct Professor

Postgraduate Researcher

Professor

Professor

Assistant Professor 
Invited Talks

$1988-1990$

1. February

Aachen, Germany

"Searches for SUPERSYMMETRY at Future $e^{+} e^{*}$ Colliders", M. Dittmar

2. March 10, 1988

High Energy Physics Seminar, Univ. of California at Irvine

"OPAL at LEP/CERN - A Gem of a Project", G. VanDalen

3. April 26, 1988

The VIII International Workshop on Photon-Photon Collisions, Shoresh, Israel

"Particle Fractions in Photon-Photon Collisions", J. Layter

4. May, 1988

University of Lisbon, Portugal

"The LEP Program, the physics potential of the four LEP experiments (a comparison of the 4 LEP experiments)", M. Dittmar

5. May, 1988

University of Madrid, Spain

"The LEP Program, the physics potential of the four LEP experiments (a comparison of the 4 LEP experiments)", M. Dittmar

6. May, 1988

University of Valencia, Spain

"The IEP Program, the physics potential of the four LEP experiments (a comparison of the 4 LEP experiments)", M. Dittmar

7. May, 1988

University of Valencia, Spain

"Searches for SUPERSYMMETRY at Future $e^{+} e^{-}$Colliders", M. Dittmar

8. May 27, 1988

San Diego Supercomputer Center, San Diego, California

"OPAL: Electron Positron Collisions at LEP", G. VanDalen

9. July 28,1988

OPAL Physics Seminar

"Summary of the VIII International Workshop on Photon-Photon Collisions", J. Layter

10. October 17,1988

Loma Linda University Physics Department

"High Energy Physics", J. Layter

11. January, 1989

Aspen Winter Meeting 1989

"LEP and OPAL Prospects for 1989", M. Dittmar 
Invited Talks - Page Two

12. January, 1989

SLAC

"LEP and OPAL Prospects for 1989", M. Dittmar

13. January, 1989

FERMILAB

"LEP and OPAL Prospects for 1989", M. Dittmar

14. January, 1989

University of Madison, Wisconsin

"Searches for SUPERSYMMETRY at Future $e^{+} e^{*}$ Colliders", M. Dittmar

15. March 8, 1989

XXIV Rencontres de Moriond: Electroweak Interactions and Unified Theories, Les Arcs, France

"Review of Close-Mass Heavy Lepton Searches", J. Keith Riles

16. March 9, 1989

OPAL Physics Seminar

"Review of Close-Mass Heavy Lepton Searches", J. Keith Riles

17. April 19, 1989

UC Riverside Physics Department Seminary

"LEP, LEP Detectors, LEP Physics", J. Layter

18. November 1, 1989

High Energy Physics Seminar, University of California at Riverside

"OPAL Results on $Z^{\cdot n}$, G. VanDalen

19. May, 1990

OPAL Physics Seminar

"Status of Neutrino Counting", J. Layter

20. May 24, 1990

Ann Arbor, Michigan, Workshop on QED Structure Functions

"Results of study of Two-Photon Backgrounds to the Neutrino-Counting Experiment", J. Keith Riles

21. January 5, 1990

American Physical Society - Division of Particles and Fields Meeting, Rice University, Houston

"Measurement of the $Z^{\circ}$ Mass and Width", G. VanDalen

22. January, 1990

U.C. Riverside High Energy Physics Seminar

"Results of OPAL Measurements of 20 Line Shape for Hadronic/Leptonic Decays, along with studies of Hadronic Event Properties", J. Keith Riles

22. January, 1990

SLAC Colloquium

"Results of OPAL Measurements of 20 Line Shape for Hadronic/Leptonic

Decays, along with studies of Hadronic Event Properties", J. Keith Riles 
Invited Talks - Page Three

23. February, 1990

Physics Department, California State Univ. at San Francisco

"First Results on the $Z^{\circ}$ at LEP", B. Shen

24. February 21, 1990

MP Division Colloquium, Los Alamos National Lab., New Mexico

"Measurement of the $Z$ Parameters with OPAL at LEP", G. VanDalen

25. March, 1990

OPAL Plenary Week

"Summary of OPAL Physics Results and Future Analysis Plans", J. Keith Riles

26. March, 1990

Institute of Physics, Academia Sinica, Taipei, Taiwan

"Recent Results and Future Development in High Energy Physics", B. Shen

27. March, 1990

Physics Department, National Taiwan University, Taipei

"New Results on Particle Physics at Accelerators in U.S. and Europe", B. Shen

28. March, 1990

Dept. of Physics and Astronomy, National Central University, Chung-Li

"Current Status and Trends in Experimental High Energy Physics", B. Shen

29. March, 1990

Dept. of Physics, National Tsing-Hwa University, Shin-Tsu, Taiwan

"Testing the Standard Model of Particle Physics", B. Shen

30. April, 1990

DESY, Meeting of the ECFA Workshop on ep collisions at LEP-LHC

"Supersymmetry Searches at HERA' (LEP-LHC ep collider)", M. Dittmar

31. May, 1990

Taiwan, Republic of China, Second International Conference for Medium and High Energy Physics

"The Future LEP-Physics-Program; some Aspects of the next 10 Years", M. Dittmar

32. May, 1990

KEK Center for High Energy Physics, Japan

"OPAL results 1989 and the future LEP-Physics Program", M. Dittmar

33. May, 1990

Institute for Nuclear Physics, Tokyo, Japan

"OPAL results 1989 and the future LEP-Physics Program", M. Dittmar

34. May, 1990

The Second International Conference Medium- and High-Energy Nuclear

Physics, National Taiwan University, Taipei, R.O.C.

"Recent Results from OPAL at LEP", B. Shen 
Invited Talks - Page Four

35. May, 1990

Physics Department, Univ. of California Los Angeles

"Study of $\mathrm{Z}^{\circ}$ at LEP", B. Shen

36. June 1,1990

University of Oregon

"Physics at the $Z$ with OPAL at LEP", G.J. VanDalen

37. June, 1990

University of Valencia, Spain

"OPAL results 1989 and the future LEP-Physics Program", M. Dittmar

38. June 27,1990

1990 Summer Study on High Energy Physics, Snowmass, Colorado, June 25-July 13, 1990

"Physics with LEP2", B. Shen 
Other Talks

$1988-1990$

1. May 3, 1990

OPAL Heavy Lepton Working Group

"Results of First Close-Mass Lepton Study", J. Kelth Riles

2. June, 1990

OPAL Collaboration Board Meeting

"A Proposal for the Management of the OPAL VAX Cluster", J. Layter

3. June 1, 1989

OPAL Physics Workshop III

"Two Photon Backgrounds to Neutrino Counting", J. Layter

4. June 3, 1989

Third OPAL Lepton Physics Workshop

"Results of First Close-Mass Lepton Study", J. Keith Riles

5. June 15, 1989

OPAl Hadron Calorimeter Meeting

"Calibration Data Structures", J. Layter

6. June 21, 1989

OPAL Heavy Lepton Working Group

"Results of further study of Closs-Mass Leptons and Electron/Muon Identification", J. Keith Riles

7. June 23,1989

OPAL Tau Pair Working Group

"Results of study of Measuring Tau Polarization in the First Year of Running", J. Keith Riles

8. August 30,1989

OPAL Heavy Lepton Working Group

"Results of Studying Track-Isolation Criteria for Selecting Anomalous Events", J. Keith RIles

9. November 15, 1989

OPAL Heavy Lepton Working Group

"Preliminary Mass Limit on Heavy Charged Lepton based on an Isolated Lepton Search", J. Keith Riles

10. March, 1990

Special Plenary-Week Offline Session

"Proposal for Compressing OPAL Data Sets", J. Keith Riles

11. March, 1990

Plenary-Week Lepton Physics Session

"Algorithm for Online Extraction of Anomalous Events", J. Keith Riles 


\section{8-1990 PUBLICATIONS}

1. W. Gorn, K. Kwong, W. Langeveld, J. Layter, C. Lindsey, S. Melnikoff, B. Shen, G. VanDalen, and 94 authors from the PEP-4 TPC/PEP-9 Two-Gamma Collaboration, Lawrence Berkeley Laboratory, UC Davis, Univ. of Calif. Instit. for Res, at Particle Acc. - Stanford, UCLA, UC Riverside, UC San Diego, UC Santa Barbara, Johns Hopkins, University of Massachusetts, NIKHEF-Amsterdam, and University of Tokyo, "Production of Four-Prong Final States in Photon-Photon Collisions", Phys. Rev. D37, 28-37 (1988).

2. W. Gorn, W. Langeveld, J. Layter, W. Lin, B. Shen, G. VanDalen, and 94 authors from the PEP-4 TPC/PEP-9 Two-Gamma Collaboration, Lawrence Berkeley Laboratory, UC Davis, Univ. of Calif. Instit. for Res. at Particle ACC. - Stanford, UCLA, UC Riverside, UC San Diego, UC Santa Barbara, Johns Hopkins, University of Massachusetts, NIKHEF-Amsterdam, and University of Tokyo, "Charmonium Production in Photon-Photon Collisions", PRL 60 (1988) 2355-2358.

3. W. Gorn, W. Langeveld, J. Layter, W. Lin, B. Shen, G. VanDalen, and 94 authors from the PEP-4 TPC/PEP-9 Two-Gamma Collaboration, Lawrence Berkeley Laboratory, UC Davis, Univ. of Calif. Instit. for Res. at Particle ACC. - Stanford, UCLA, UC Riverside, UC San Diego, UC Santa Barbara, Johns Hopkins, University of Massachusetts, NIKHEF-Amsterdam, and University of Tokyo, "Formation of Spin-One Mesons by Photon-Photon Fusion", PR D38 (1988) 1-18.

4. D. Beavis, S.Y. Fung, B. Gorn, R. Poe, G. VanDalen and 24 authors from the Brookhaven National Laboratory, Los Alamos National Lab, Temple University, UC Los Alamos, UC Riverside, University of Iowa, University of New Mexico, Valparaiso University, "Analysis of the Exclusive Interaction $\nu_{\mu}{ }^{12} \mathrm{C} \rightarrow_{\mu}{ }^{-12} \mathrm{~N}$ Near Threshold", AIP Conference Proceedings 176 on Intersections Between Particle and Nuclear Physics, Rockport, ME, 912-917 (1988).

5. W. Gorn, W. Langeveld, J. Layter, W. Lin, B. Shen, G. VanDalen, and 94 authors from the PEP-4 TPC/PEP-9 Two-Gamma Collaboration, Lawrence Berkeley Laboratory, UC Davis, Univ. of Calif. Instit. for Res. at Particle ACC. - Stanford, UCLA, UC Riverside, UC San Diego, UC Santa Barbara, Johns Hopkins, University of Massachusetts, NIKHEF-Amsterdam, and University of Tokyo, " $f_{1}(1285)$ Formation in Photon-Photon Fusion", Phys. Lett. B209 (1988) 107-112.

6. M. Daoudi, W. Gorn, W. Langeveld, J. Layter, W. Lin, B. Shen, G. VanDalen, and 94 authors from the PEP-4 TPC/PEP-9 Two-Gamma Collaboration, Lawrence Berkeley Laboratory, UC Davis, Univ. of Calif. Instit. for Res. at Particle Acc. - Stanford, UCLA, UC Riverside, UC San Diego, UC Santa Barbara, Johns Hopkins, University of Massachusetts, NIKHEF-Amsterdam, and University of Tokyo, "Charged Hadron Inclusive Cross Sections and Fractions in $e^{+} e^{-}$Annihilation at $\sqrt{s}-29 \mathrm{GeV}^{n}$, Phys. Rev. Lett. 61, 12631266 (1988). 
7. M. Daoudi, W. Langeveld, J.G. Layter, W.T. Lin, B.C. Shen, G.J. VanDalen and 76 authors from TPC/Two-Gamma Collaboration, UCR and 11 other institutions, "Evidence of Soft and Collinear Gluon Emission in $e^{+} e^{-}$ Hadronic Events", Z. Phys. C44, 357-364 (1989).

8. M. Daoudi, W. Langeveld, J. Layter, W. Lin, B. Shen, and 94 authors from the PEP-4 TCP/PEP-9 Two-Gamma Collaboration, Lawrence Berkeley Laboratory, UC Davis, Univ. of Calif. Instit. for Res. at Particle Acc. - Stanford, UCLA, UC Riverside, UC San Diego, UC Santa Barbara, Johns Hopkins, University of Massachusetts, NIKHEF-Amsterdam, and University of Tokyo, "Exclusive Production of $\mathrm{pp} \pi^{+} \pi$ in Photon-Photon Collisions", Physical. Review D40, 2772 (1989).

9. M. Dittmar, W. Gorn, E. Heflin, C. Ho, E. Jin, W.J. Larson, J.G. Layter, J. Ma, B.O. Neill, H. Oh, K. Riles, B.C. Shen, G.J. VanDalen, H. Wang, Y. Yang and 262 authors from the OPAL Collaboration, Univ. Birmingham, Univ. di Bologna, INFN, Univ. Bonn, UC Riverside, Cavendish Lab, Carleton University, CERN, Univ. of Chicago, Albert Ludwigs Univ., Univ. Heidelberg, Queen Mary and Westfield College, Birkbeck College, Univ. London, Univ. Manchester, Univ. Maryland, Univ. Montreal, Herzberg Inst. of Astrophysics, Rutherford Appleton Lab, CEN Saclay, TechnionIsrael Inst. of Tech., Tel Aviv Univ., Univ. of Tokyo, Brunel Univ., Weizmann Institute of Sc1., "Measurement of the $\mathrm{Z}^{\circ}$ Mass and Width with the OPAL Detector at LEP", Phys. Lett. B231, 530-538 (1989).

10. K. Riles, I. Juricic, et al., "Bose-Einstein Correlations in $e^{+} e^{-}$ Collisions", Phys. Rev. D39, 1 (1989).

11. K. Riles, D. P. Stoker, et al., "Limits on New Lepton Pairs ( $\left.L^{-} L^{*}\right)$ with Arbitrary Neutrino Mass", Phys. Rev. D39, 1811 (1989).

12. K. Riles, D. Karlen, et al., "Measurement of Single and Double Radiative Low $q^{2}$ Bhabha Scattering at $E_{c . m}=29 \mathrm{GeV}^{\prime \prime}$, Phys. Rev. D39, 1861 (1989).

13. K. Riles, R.A. Ong, et al., "A Refined Measurement of the B Hadron Lifetime", Phys. Rev. Lett. 62, 1236 (1989).

14. K. Riles, S. Klein, et al., " $\Lambda^{+}$, Production and Semileptonic Decay in 29 GeV e $e^{-}$Annihilation", Phys. Rev. Lett. 62, 2444 (1989).

15. K. Riles, "Review of Close-Mass Heavy Lepton Searches", Proceedings of XXIV Rencontres de Moriond: Electroweak Interactions and Unified Theories, Les Arcs, France, March 5-12, 189 (1989).

16. K. Riles, S. Bethke, et al., "Studies of Jet Production Rates in $\mathrm{e}^{+} \mathrm{e}^{\text {- }}$ Annihilation at $E_{\text {c.m. }}=29 \mathrm{GeV}^{\prime \prime}$, Physics $\underline{\mathrm{C} 43}, 325$ (1989).

17. K. Riles, G.S. Abrams, et al., "The Mark II Detector for the SLC", Nucl. Instr. Meth. A281, 55 (1989).

18. K. Riles, G. S. Abrams, et al., "Initial Measurements of $Z$ Boson Resonance Parameters in $e^{+} e^{-}$Annihilation", Phys. Rev. Lett. $\underline{63}, 724$ (1989). 
19. K. Riles, A. Snyder, et al., "Search for B Decay to Higgs Bosons for Higgs Boson Masses Between $50-\mathrm{MeV} / \mathrm{C}^{2}$ and $210 \mathrm{MeV} / \mathrm{c}^{21 "}$, Phys. Lett. B229, 169 (1989).

20. K. Riles, G.S. Abrams, et al., "First Measurements of Hadronic Decays of the $\mathrm{Z}$ Boson", Phys. Rev. Lett. 63, 1558 (1989).

21. K. Riles, G.S. Abrams, et al., "Measurements of $Z$ Boson Resonance Parameters in $e^{+} e^{-}$Annihilation", Phys. Rev. Lett. 63, 2173 (1989).

22. K. Riles, G.S. Abrams, et al., "Searches for New Quarks and Leptons Produced in Z Boson Decay", Phys. Rev. Lett. 63, 2780 (1989).

23. K. Riles, G.S. Abrams, et al., "Measurement of $Z^{\circ}$ Decays into Lepton Pairs", Phys. Rev. Lett. 63, 2780 (1989).

24. K. Riles, S. Komamiya, et al., "Searches for Nonminimal Higgs Bosons from a Virtual Z Decaying into a Muon Pair at PEPn, Phys. Red. D40, 721 (1989).

25. M. Daoudi, W.G.J. Langeveld, J.G. Layter, W. I. Iin, B. C. Shen, and 75 authors from the TPC/Two Gamma Collaboration, Lawrence Berkeley Laboratory, UC Davis, Univ. of Calif. Instit. for Res. at Particle Acc. Stanford, UCLA, UC Riverside, UC San Diego, UC Santa Barbara, Ames Laboratory - Iowa State University, Johns Hopkins, Univeristy of Massachusetts, National Inst. for Nuclear and High Engergy Physics Amsterdam, and University of Tokyo, "Investigation of the Electromagnetic Structure of $\eta$ and $\eta^{\prime}$ Mesons by Two-Photon Interactions", Phys. Rev. Lett. 64, $172-174$ (1990).

26. M. Daoudi, W. Langeveld, J. Layter, W. Lin, B. Shen, and 94 authors from the PEP-4 TPC/PEP-9 Two-Gamma Collaboration, Lawrence Berekely Laboratory, UC Davis, Univ. of Calif. Instit. for Res. at Particle Acc. - Stanfrod, UCLA, UC Riverside, UC San Diego, UC Santa Barbara, Johns Hokings, University of Massachusetts, NIKHEF-Amsterdam, and University of Tokyo, "A Measurement of the Total Hadronic Cross Section in Tagged Gamma-Gamma Reactions", Physical Review D41, 2667-2674 (1990).

27. M. Daoudi, J. Layter, J. K. Riles, and B. Shen, "Study of the All Neutral Final State of $f_{2}$ (1270) Produced in Two-Photon Collision as a Background to Radiative Neutrino Counting on the $\mathrm{Z}^{*}$ Resonance" , in Proceedings of the Workshop on QED Structure Functions", Ann Arbor, Michigan May 22-25, AIP201, editor G. Bonvicini (1989).

28. M. Dittmar, W. Gorn, E. Heflin, C. Ho, E. Jin, W.J. Larson, J.G. Layter, J. Ma, B.O. Neill, H. Oh, K. Riles, B.C. Shen, G.J. VanDalen, H. Wang, Y. Yang and 262 authors from the OPAL Collaboration, Univ. Birmingham, Univ. di Bologna, INFN, Univ. Bonn, UC Riverside, Cavendish Lab, Carleton University, CERN, Univ. of ChIcago, Albert Ludwigs Univ., Univ. Heidelberg, Queen Mary and Westfield College, Birkbeck College, Univ. London, Univ. Manchester, Univ. Maryland, Univ. Montreal, Herzberg Inst. of Astrophysics, Rutherford Appleton Lab, CEN Saclay, Technion-Israel Inst. of Tech., Tel Aviv Univ., Univ. of Tokyo, Brunel Univ., Weizmann Institute of Sci., "Measurement of the Decay of the $Z^{\bullet}$ into Lepton Pairs", Phys. Lett, B235, 379-388 (1990). 
29. M. Dittmar, W. Gorn, E. Heflin, C. Ho, E. Jin, W.J. Larson, J.G. Layter, J. Ma, B.O. Neill, H. Oh, K. Riles, B.C. Shen, G.J. VanDalen, H. Wang, Y. Yang and 262 authors from the OPAL Collaboration, Univ. Birmingham, Univ. di Bologna, INFN, Univ. Bonn, UC Riverside, Cavendish Lab, Carleton University, CERN, Univ. of Chicago, Albert Ludwigs Univ., Univ. Heidelberg, Queen Mary and Westfield College, Birkbeck College, Univ. London, Univ. Manchester, Univ. Maryland, Univ. Montreal, Herzberg Inst. of Astrophysics, Rutherford Appleton Lab, CEN Saclay, Technion-Israel Inst. of Tech., Tel Aviv Univ., Univ. of Tokyo, Brunel Univ., Weizmann Institute of Sci., "A Study of Jet Production Rates and a Test of QCD on the $\mathrm{Z}^{\circ}$ Resonance", Phys. Lett., B235, 389 (1990).

30. M. Dittmar, W. Gorn, E. Heflin, C. Ho, E. Jin, W.J. Larson, J.G. Layter, J. Ma, B.O. Neill, H. Oh, K. Riles, B.C. Shen, G.J. VanDalen, H. Wang, Y. Yang and 262 authors from the OPAL Collaboration, Univ. Birmingham, Univ. di Bologna, INFN, Univ. Bonn, UC Riverside, Cavendish Lab, Carleton University, CERN, Univ. of Chicago, Albert Ludwigs Univ., Univ. Heidelberg, Queen Mary and Westfield Colllege, Birkbeck College, Univ. London, Univ. Manchester, Univ. Maryland, Univ. Montreal, Herzberg Inst. of Astrophysics, Rutherford Appleton Lab, CEN Saclay, Technion-Israel Inst. of Tech., Tel Aviv Univ., Univ. of Tokyo, Brunel Univ., Weizmann Institute of Sci., "A Search for Top and $b^{\prime}$ Quarks in Hadronic $z^{\circ}$ Decays", Phys. Lett. B236, 364 (1990).

31. M. Dittmar, W. Gorn, E. Heflin, C. Ho, E. Jin, W.J. Larson, J.G. Layter, J. Ma, B.O. Neill, H. Oh, K. Riles, B.C. Shen, G.J. VanDalen, H. Wang, Y. Yang and 262 authors from the OPAL Collaboration, Univ. Birmingham, Univ. di Bologna, INFN, Univ. Bonn, UC Riverside, Cavendish Lab, Carleton University, CERN, Univ. of Chicago, Albert Ludwigs Univ., Univ. Heidelberg, Queen Mary and Westfield College, Birkbeck College, Univ. London, Univ. Manchester, Univ. Maryland, Univ. Montreal, Herzberg Inst. of Astrophysics, Rutherford Appleton Lab, CEN Saclay, Technion-Israel Inst. of Tech., Tel Aviv Univ., Univ. of Tokyo, Brunel Univ., Weizmann Institute of Sci., "A Search for Acoplanar Pairs of Leptons or Jets in $Z^{\circ}$ Decays", Phys. Lett. B240, 261-270 (1990).

32. M. Dittmar, W. Gorn, E. Heflin, C. Ho, W.J. Larson, J.G. Layter, J. Ma, B.O. Neill, H. Oh, K. Riles, B.C. Shen, G.J. VanDalen, Y. Yang and 262 authors from the OPAL Collaboration, Univ. Birmingham, Univ. di Bologna, INFN, Univ. Bonn, UC Riverside, Cavendish Lab, Carleton University, CERN, Univ. of Chicago, Albert Ludwigs Univ., Univ. Heidelberg, Queen Mary and Westfield College, Birkbeck College, Univ. London, Univ. Manchester, Univ. Maryland, Univ. Montreal, Herzberg Inst. of Astrophysics, Rutherford Appleton Lab, CEN Saclay, Technion-Israel Inst. of Tech., Tel Aviv Univ., Univ. of Tokyo, Brunel Univ., Weizmann Institute of Sci., "A Direct Search for New Charged Heavy Leptons at LEP", Phys. Lett. B240, 250-260 (1990). 
33. M. Dittmar, W. Gorn, E. Heflin, C. Ho, E. Jin, W.J. Larson, J.G. Layter, J. Ma, B.O. Neill, H. Oh, K. Riles, B.C. Shen, G.J. VanDalen, H. Wang, Y. Yang and 262 authors from the OPAL Collaboration, Univ. Birmingham, Univ. di Bologna, NFN, Univ. Bonn, UC Riverside, Cavendish Lab, Carleton University, CERN, Univ. of Chicago, Albert Ludwigs Univ., Univ. Heidelberg, Queen Mary and Westfield College, Birkbeck College, Univ. London, Univ. Manchester, Univ. Maryland, Univ. Montreal, Herzberg Inst. of Astrophysics, Rutherford Appleton Lab, CEN Saclay, Technion-Israel Inst. of Tech., Tel Aviv Univ., Univ. of Tokyo, Brunel Univ., Weizmann Institute of ScI., "Mass Limits for a Standard Model Higgs Boson in $\mathrm{e}^{+} e^{-}$ Collision at LEP", Phys. Lett. B236, 224-232 (1990).

34. M. Dittmax, พ. Gorn, E. Heflin, C. Ho, W.J. Larson, J.G. Layter, J. Ma, B.O. Neill, H. Oh, K. Riles, B.C. Shen, G.J. VanDalen, Y. Yang and 262 authors from the OPAL Collaboration, Univ. Birmingham, Univ. di Bologna, INFN, Univ. Bonn, UC Riverside, Cavendish Lab, Carleton University, CERN, Univ, of Chicago, Albert Ludwigs Univ., Univ. Heidelberg, Queen Mary and Westfield College, Birkbeck College, Univ. London, Univ. Manchester, Univ. Maryland, Univ. Montreal, Herzberg Inst. of Astrophysics, Rutherford Appleton Lab, CEN Saclay, Technion-Israel Inst. of Tech., Tel Aviv Univ., Univ. of Tokyo, Brunel Univ., Weizmann Institute of Sci., "A Study of the Reaction $e^{+} e^{-} \rightarrow \gamma \gamma$ at LEP, Phys. Lett. B241, 133-140 (1990).

35. M. Dittmar, W. Gorn, E. Heflin, C. Ho, E. Jin, W.J. Larson, J.G. Layter, J. Ma, B.O. Neill, H. Oh, K. Riles, B.C. Shen, G.J. VanDalen, H. Wang, Y. Yang and 262 authors from the OPAL Collaboration, Univ. Birmingham, Univ. di Bologna, INFN, Univ. Bonn, UC Riverside, Cavendish Lab, Carleton University, CERN, Univ. of Chicago, Albert Ludwigs Univ., Univ. Heidelberg, Queen Mary and Westfield College, Birkbeck College, Univ. London, Univ. Manchester, Univ. Maryland, Univ. Montreal, Herzberg Inst. of Astrophysics, Rutherford Appleton Lab, CEN Saclay, Technion-Israel Inst. of Tech., Tel Aviv Univ., Univ. of Tokyo, Brunel Univ., Weizmann Institute of Sci., "A Combined Analysis of the Hadronic and Leptonic Decays of the $Z^{\circ} "$, Phys. Lett. B240, 497-512 (1990).

36. M. Dittmar, A. Santamaria, M.C. Gonzalez-Garcia and J.W.F. Valle, "Production Mechanisms and Signatures of Isosinglet Neutral Heavy eptons in $Z^{\circ}$ Decays", Production Mechanisms and Signatures, Nuclear Physics B332, $1-19$ (1990).

37. M. Dittmar, "Physics Possibilities", (working group report, 41 members) Contribution to the CERN report High Luminosity LEP; May (1990).

38. M. Dittmar, F. Boudjema, R. Casalbuoni, P. Chiappeta, D. Cocoliechio, F. Peruglio, B. Gavela (convener), R. Kleiss, S. Mouharik, G. Ridolfi, J.W.F. Valle, F. Zwiner (converner), "Higgs and New Physics" (subworking group), May (1990).

39. M. Dittmar and J.W.F. Valle, "Heavy Singlet Neutral Leptons, Flavour and C1 Violation on the $\mathrm{Z}$ Peak", Preliminary Report to the LEPC from the Working Group on High-Luminosities at LEP, to be published in the High Luminosity LEP Report, May (1990). 
40. M. Dittmar and D. Cocolicchio, "The Radiative and Hadronic Flavour Changing Decays of the $\mathrm{Z}^{n}$, CERNTH-5753/90 Contribution to the CERN Report High Luminosity LEP, May (1990).

\section{Submitted}

1. G. J. VanDalen, " $\mathrm{Z}^{\bullet}$ Mass and Width and the Number of Neutrinos" to appear in Proceedings of DPF90, January 3-6, 1990, Rice University, Houston.

2. M. Dittmar, W. Gorn, E. Heflin, C. Ho, W. J. Lawson, J. G. Layter, J. Ma, B. P. O'Neill, H. Oh, K. Riles, B. C. Shen, G. J. VanDalen, Y. Yang et al., "A Search for Technipions and Charged Higgs Bosons at LEP", CERNEP/90-38, submitted to Phys. Lett.

3. M. Dittmar, W. Gorn, E. Heflin, C. Ho, W. J. Larson, J. G. Layter, M. Ma, B. P. O'Neill, H. Oh, K. Riles, B. C. Shen, G. J. VanDalen, Y. Yang et al., "A Measurement of Global Event Shape Distributions in the Hadronic Decays of the $Z^{\circ}$, CERN-EP/90-48, submitted to Zeitschrift f. Physik.

4. M. Dittmar, W. Gorn, E. Heflin, C. Ho, W. J. Larson, J. G. Layter, J. Ma, B. P. O'Neill, H. Oh, K. Riles, B. C. Shen, G. J. VanDalen, Y. Yang, et a. , "Search for Excited Leptons at LEP", CERN-EP/90-49, submitted to Phys. Lett.

5. M. Dittmar, W. Gorn, E. Heflin, C. Ho, W. J. Larson, J. G. Layter, J. Ma, B. P. O'Neill, H. Oh, K. Riles, B. C. Shen, G. J. VanDalen, Y. Yang et al., "Evidence for Final State Photons in Multihadronic Decays of the $Z^{\bullet}$, CERN-EP/90-55, submitted to Phys. Lett.

6. M. Dittmar, W. Gorn, E. Heflin, C. Ho, W. J. Larson, J. G. Layter, J. Ma, B. P. O'Neill, H. Oh, K. Riles, B. C. Shen, G. J. VanDalen, Y. Yang et al., "Limits on Neutral Heavy Lepton Production from $Z^{\circ}$ Decay", CERNEP/90-72, submitted to Phys. Lett.

7. K. Riles, J.F. Kral, et al., "Measurement of the $b$ anti-b Fraction in Hadronic Z Decays", to appear in Phys. Rev. Lett., SLAC--PUB5147, (1989).

8. K. Riles, D.Y. Wu, et al., "Radiative Tau Production and Decay", to appear in Phys. Rev. D, SLAC--PUB5128 (1989).

9. K. Riles, C. Jung, et al., "Search for Long-Lived Massive Neutrinos in Z Decays", to appear in Phys. Rev. Lett., SLAC--PUB--5136 (1989).

10. M. Dittmar, "The Future LEP-Physics Program; Some Aspects of the Next 10 Years", to appear in Proceedings of the Second International Conference on Medium-and High-Energy Nuclear Physics, Taiwan (1990).

11. B. C. Shen, "Recent Results from OPAL at LEP", to appear in Proceedings of the Second International Conference on Medium and High Energy Nuclear Physics, Taiwan (1990). 
To Be Submitted

1. M. Dittmar, W. Gorn, E. Heflin, C. Ho, W. J. Larson, J. G. Layter, J. Ma, B. P. O'Neill, H. Oh, K. Riles, B. C. Shen, G. J. VanDalen, Y. Yang et al., "Analysis of $Z^{\circ}$ Couplings to Charged Leptons", CERN-EP/90-xx, to be submitted to Phys. Lett.

2. M. Dittmar, W. Gorn, E. Heflin, C. Ho, W. J. Larson, J. G. Layter, J. Ma, B. P. O'Neill, H. Oh, K. Riles, B. C. Shen, G. J. VanDalen, Y. Yang et al., "A Study

of Coherence of Soft Gluons in Hadron Jets", CERN-EP/90-xx, to be submitted to Phys. Lett.

3. M. Dittmar, W. Gorn, E. Heflin, C. Ho, W. J. Larson, J. G. Layter, J. Ma, B. P. O'Neill, H. Oh, K. Riles, B. C. Shen, G. J. VanDalen, Y. Yang et al., "A Study of the Angular Correlations in 4-Jet Final states of hadronic $Z^{\circ}$ Decays", CERN-EP/90-xx, to be submitted to Phys. Lett.

4. M. Dittmar, W. Gorn, E. Heflin, C. Ho, E. Jin, W. J. Larson, J. G. Layter, J. Ma, B. P. O'Neill, H. Oh, K. Riles, B. C. Shen, G. J. Vandalen, H. Wang, Y. Yang et al., "The OPAL Detector at LEP", CERN-EP/90-xx, to be submitted to Nucl. Instr. and Meth.

5. M. Dittmar, W. Gorn, H. Heflin, C. Ho, W. J. Larson, J. G. Layter, J. Ma, B. P. O'Neill, H. Oh, K. Riles, B. C. Shen, G. J. VanDalen, Y. Yang et al., "Direct Search for Neutralino Production at LEP", CERN-EP/90-XX, to be submitted to Physics Letters.

6. M. Daoudi, W. Langeveld, J. Layter, W. Lin, B. Shen, G. VanDalen, and 94 authors from the PEP-4 TPC/PEP-9 Two-Gamma Collaboration, Lawrence Berkeley Laboratory, UC Davis, Univ. of Calif. Instit. for Res. at Particle Acc. - Stanford, UCLA, UC Riverside, UC San Diego, UC Santa Barbara, Johns Hopkins, University of Massachusetts, NIKHEF-Amsterdam, and University of Tokyo, "Inclusive $D^{* \pm}$ Production in Photon-Photon Collisions", to be submitted to Physical Review.

7. M. Daoudi, W. Langeveld, J. Layter, W. Lin, B. Shen, G. VanDalen, and 94 authors from the PEP-4 TPC/PEP-9 Two-Gamma Collaboration, Lawrence Berkeley Laboratory, UC Davis, Univ. of Calif. Instit. for Res. at Particle Acc. - Stanford, UCLA, UC Riverside, UC San Diego, UC Santa Barbara, Johns Hopkins, University of Massachusetts, NIKHEF-Amsterdam, and University of Tokyo, "Measurement of the Photon Structure Function $F_{2}$ between $10<Q^{2}<60 \mathrm{GeV}^{2 n}$, to be submitted to Zeitschrift für Phyzik.

8. M. Daoudi, W. Langeveld, J. Layter, W. Lin, B. Shen, G. VanDalen, and 94 authors from the PEP-4 TPC/PEP-9 Two-Gamna Collaboration, Lawrence Berkeley Laboratory, UC Davis, Univ. of Calif. Instit. for Res, at Particle Acc. - Stanford, UCLA, UC Riverside, UC San Diego, UC Santa Barbara, Johns Hopkins, University of Massachusetts, NIKHEF-Amsterdam, and University of Tokyo, "Charged Hadron Production in $e^{+} e^{-}$Annihilation at $\sqrt{s}$ - $29 \mathrm{GeV}^{\prime \prime}$, to be submitted to Physical Review D. 
9. M. Daoudi, W. Langeveld, J. Layter, W. Lin, B. Shen, G. VanDalen, and 94 authors from the PEP-4 TPC/PEP-9 Two-Gamma Collaboration, Lawrence Berkeley Laboratory, UC Davis, Univ. of Calif. Instit. for Res. at Particle ACC. - Stanford, UCLA, UC Riverside, UC San Diego, UC Santa Barbara, Johns Hopkins, University of Massachusetts, NIKHEF-Amsterdam, and University of Tokyo, "Study of Two-Photon Production of $\rho \omega$ in $e^{+} e^{\text {- }}$ Interactions", to be submitted to Physical Review D.

10. M. Daoudi, W. Langeveld, J. Layter, W. Lin, B. Shen, G. VanDalen, and 94 authors from the PEP-4 TPC/PEP-9 Two-Gamma Collaboration, Lawrence Berkeley Laboratory, UC Davis, Univ. of Calif. Instit. for Res. at Particle AcC. - Stanford, UCLA, UC Riverside, UC San Diego, UC Santa Barbara, Johns Hopkins, University of Massachusetts, NIKHEF-Amsterdam, and University of Tokyo, "Production of $\eta$ and $\eta$ ' Mesons in Single-Tagged Two-Photon Interactions", to be submitted to Physical Review D.

11. M. Daoudi, W. Langeveld, J. Layter, W. Lin, B. Shen, G. VanDalen, and 94 authors from the PEP-4 TPC/PEP-9 Two-Gamma Collaboration, Lawrence Berkeley Laboratory, UC Davis, Univ. of Calif. Instit. for Res. at Particle ACC. - Stanford, UCLA, UC Riverside, UC San Diego, UC Santa Barbara, Johns Hopkins, University of Massachusetts, NIKHEF-Amsterdam, and University of Tokyo, "Limits on Heavy Leptons with Massive Neutrinos".

12. M. Daoudi, W. Langeveld, J. Layter, B. Shen, G. VanDalen, and 95 authors from the PEP-4 TPC/PEP-9 Two-Gamma Collaboration, Lawrence Berkeley Laboratory, UC Davis, Univ. of Calif. Instit. for Res. at Particle Acc. Stanford, UCLA, UC Riverside, UC San Diego, UC Santa Barbara, Johns Hopkins, University of Massachusetts, NIKHEF-Amsterdam, and University of Tokyo, "A Measurement of A2(1320) Formation in Photon-Photon Collisions".

13. M. Daoudi, W. Langeveld, J. Layter, W. Lin, B. Shen, G. VanDalen, and 94 authors from the PEP-4 TPC/PEP-9 Two-Gamma Collaboration, Lawrence Berkeley Laboratory, UC Davis, Univ. of Calif. Instit. for Res. at Particle ACC. - Stanford, UCLA, UC Riverside, UC San Diego, UC Santa Barbara, Johns Hopkins, University of Massachusetts, NIKHEF-Amsterdam, and University of Tokyo, "Test of Spin Dependence in Charm Quark Fragmentation to $D^{*+n}$, to be submitted to Physical Review D.

14. M. Daoudi, W. Langeveld, J. Layter, W. Lin, B. Shen, G. VanDalen, and 94 authors from the PEP-4 TPC/PEP-9 Two-Gamma Collaboration, Lawrence Berkeley Laboratory, UC Davis, Univ. of Calif. Instit. for Res. at Particle Acc. - Stanford, UCLA, UC Riverside, UC San Diego, UC Santa Barbara, Johns Hopkins, University of Massachusetts, NIKHEF-Amsterdam, and University of Tokyo, "Jets in Photon-Photon Interactions".

15. M. Daoudi, W. Langeveld, J. Layter, W. Lin, B. Shen, G. VanDalen, and 94 authors from the PEP-4 TPC/PEP-9 Two-Gamma Collaboration, Lawrence Berkeley Laboratory, UC Davis, Univ. of Calif. Instit. for Res. at Particle ACC. - Stanford, UCLA, UC Riverside, UC San Diego, UC Santa Barbara, Johns Hopkins, University of Massachusetts, NIKHEF-Amsterdam, and University of Tokyo, "Inclusive Production of Vector Mesons in $\mathrm{e}^{+} \mathrm{e}^{\text {- }}$ Annihilation at $\checkmark s=29 \mathrm{GeV}$ ". 


\section{$2.2 \quad \mathrm{TPC} / 2 \gamma$ Experiment at PEP}

\subsubsection{Introduction}

The upgraded TPC $/ 2 \gamma$ facility is just beginning to collect data in its high luminosity era. The upgrade of the detector consisted mainly of the installation around a $4 \mathrm{~cm}$ radius beam pipe of a high precision vertex tracker that provides an impact parameter resolution of $90 \mu \mathrm{m}$ for a $1 \mathrm{GeV}$ track. Furthermore, the removal of material in front of the TPC has helped substantially reduce backgrounds (from photon conversions) inside its tracking volume. This resulted in an important improvement in the momentum resolution :

$$
\sigma_{p}^{2}=(0.01)^{2}+(0.003 p[\mathrm{GeV} / \mathrm{c}])^{2} .
$$

The PEP ring is also undergoing a major upgrade which when fully completed will make it capable of delivering a luminosity of $10^{32} \mathrm{~cm}^{-2} \mathrm{sec}^{-1}$. The beam $\beta_{y}^{*}$ was reduced from $11 \mathrm{~cm}$ to $5.5 \mathrm{~cm}$ and the ring is now operating with the two beams colliding in one interaction region only and separated in the remaining five. This configuration was fully tested during a short run in the Fall of 1988 . During that run, the amount of current in the ring was limited by a heating problem in the vertex detector which as a result held the luminosity to a maximum of $6 \times 10^{31} \mathrm{~cm}^{-2} \mathrm{sec}^{-1}$ and only about $20 \mathrm{pb}^{-1}$ of data were logged. This problem has since been resolved by installing a new cooling system for the vertex chamber.

In addition, fast switching between SLC mode and PEP mode for the LINAC has been developed to allow concurrent operation of the two machines under the restriction that the impact of PEP on SLC would not exceed $20 \%$. This system has now been fully commissioned and switch times as fast as one hour have been achieved.

The 1990 data run began in the middle of May. However, due to successive hardware failures in both the LINAC and PEP, and also because highest priority was given to getting Mark II back into operation at the beginning of July, normal running conditions of the TPC/2 $\gamma$ experiment have not been reached yet; only about $4 \mathrm{pb}^{-1}$ of data have been written to tape, and the highest instantaneous luminosity reached so far is $4 \times 10^{31} \mathrm{~cm}^{-2} \mathrm{sec}^{-1}$. The present schedule calls for two fills per day for PEP. All the detector components are currently functioning properly.

\subsubsection{9-90 Progress}

During the last academic year the vertex chamber has been fully integrated in the detector complex, resulting in a marked improvement in both tracking and triggering. In addition, many improvements to the experiment have been made, particularly in the areas of online software and diagnostics. A number of ongoing analysis projects 
Table 2.1: Measurements of the $f_{2}^{\prime} \gamma \gamma$-width in the untagged $K_{s}^{0} K_{s}^{0}$ and $K^{+} K^{-}$ channels.

\begin{tabular}{|c|c|c|}
\hline Exp & anel & $\Gamma_{\gamma \gamma}(\mathrm{keV})$ \\
\hline TASSO & $K^{0} K^{0} ; K^{+} K^{-}$ & $0.11 \pm 0.02 \pm 0.04$ \\
\hline PLUTO & $K_{s}^{0} K_{s}^{0}$ & $0.10_{-0.03}^{+0.04}{ }_{-0.02}^{+0.03}$ \\
\hline CEL & $K_{s}^{0} K$ & $0.11_{-0.02}^{+0.03} \pm 0.02$ \\
\hline ARG & $K^{+} K^{-}$ & $0.130_{-0.024}^{+0.034}$ \\
\hline $\mathrm{TPC} / 2 \gamma$ & $K_{s}^{0} K_{s}^{0}$ & $0.12 \pm 0.04$ \\
\hline
\end{tabular}

have been carried through; some of them have already been published[1][2][3][4] and others are nearly ready to be submitted for publication [5][6][7][8][9][10].

Mourad Daoudi of UCR has continued his analysis of the $f_{2}^{\prime}$ meson in tagged $\gamma \gamma$ collisions[11], studying the reaction:

$$
e^{+} e^{-} \rightarrow e^{+} e^{-} \gamma \gamma^{*} \rightarrow e^{+} e^{-} f_{2}^{\prime} \rightarrow e^{+} e^{-} K^{+} K^{-},
$$

where one of the scattered $e^{ \pm}$is detected. Table 2.1 shows a summary of the present measurements of the $f_{2}^{\prime} \gamma \gamma$-width including the TPC/2 $\gamma$ preliminary result in the $K_{s}^{0} K_{s}^{0}$ channel. All these measurements were performed in the no-tag mode and all of them assume a helicity 2 for the $f_{2}^{\prime}$. (PLUTO has actually measured it.)

The TPC/ $2 \gamma$ data sample that was used corresponds to an integrated luminosity of $115 \mathrm{pb}^{-1}$. Events with exclusively two charged tracks (one positive and one negative) identified as kaons by simultaneously measuring their momentum and energy loss $(\mathrm{dE} / \mathrm{dx})$ in the TPC and one tag in the forward spectrometer were selected. The total transverse momentum was required to be $\leq 0.2 \mathrm{GeV} / \mathrm{c}$ to ensure the exclusivity of the final state, and events that had energy depositions in the central calorimeters that were not associated with the charged tracks were rejected.

After all selection cuts 135 events remained. In Figure 2.1 the $\mathrm{dE} / \mathrm{dx}$ of a track is plotted versus its momentum, the solid curves correspond to the theoretical predictions for the different particle species. Figure 2.2 shows the $\mathrm{K}^{+} \mathrm{K}^{-}$invariant mass for these events. A clear peak at around $1.5 \mathrm{GeV}$ can be seen which is an indication of $f_{2}^{\prime}$ production. The shaded histogram corresponds to the Monte Carlo expectation for a pure $f_{2}^{\prime}(1525)$ meson whose $\gamma \gamma$-width was assumed to be $0.1 \mathrm{keV}$ and whose helicity was assumed to be equal to 2 . Another wider enhancement can be seen at around $1.3 \mathrm{GeV}$; this corresponds to the constructive interference of the $f_{2}(1270)$ and $a_{2}^{0}(1320)$, the other two isoscalar neutral flavor members of the tensor meson nonet $\left(2^{++}\right)$which decay also into $K^{+} K^{-}$. From Table 2.2, we can deduce that even though both the $f_{2}$ and $a_{2}^{0}$ have smaller branching ratios into $K \bar{K}$ than the $f_{2}^{\prime}$, they are much wider and have stronger couplings to two photons, so that their contribution to the $K \bar{K}$ mass spectrum is comparable to that of the $f_{2}^{\prime}$ as can be seen from the data. 


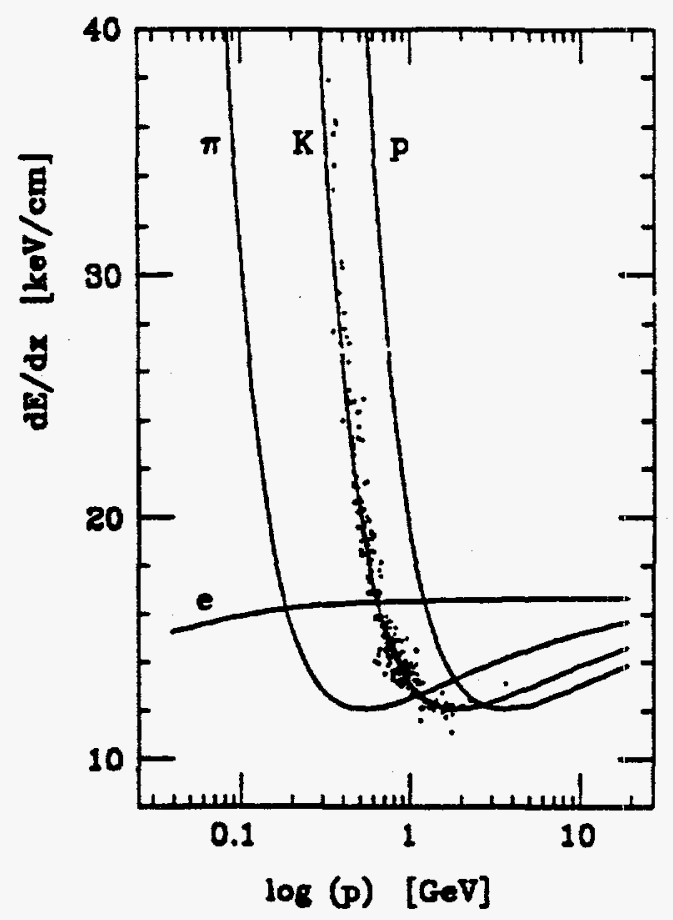

Figure 2.1: Energy loss vs. momentum. The curves represent the different particle types.

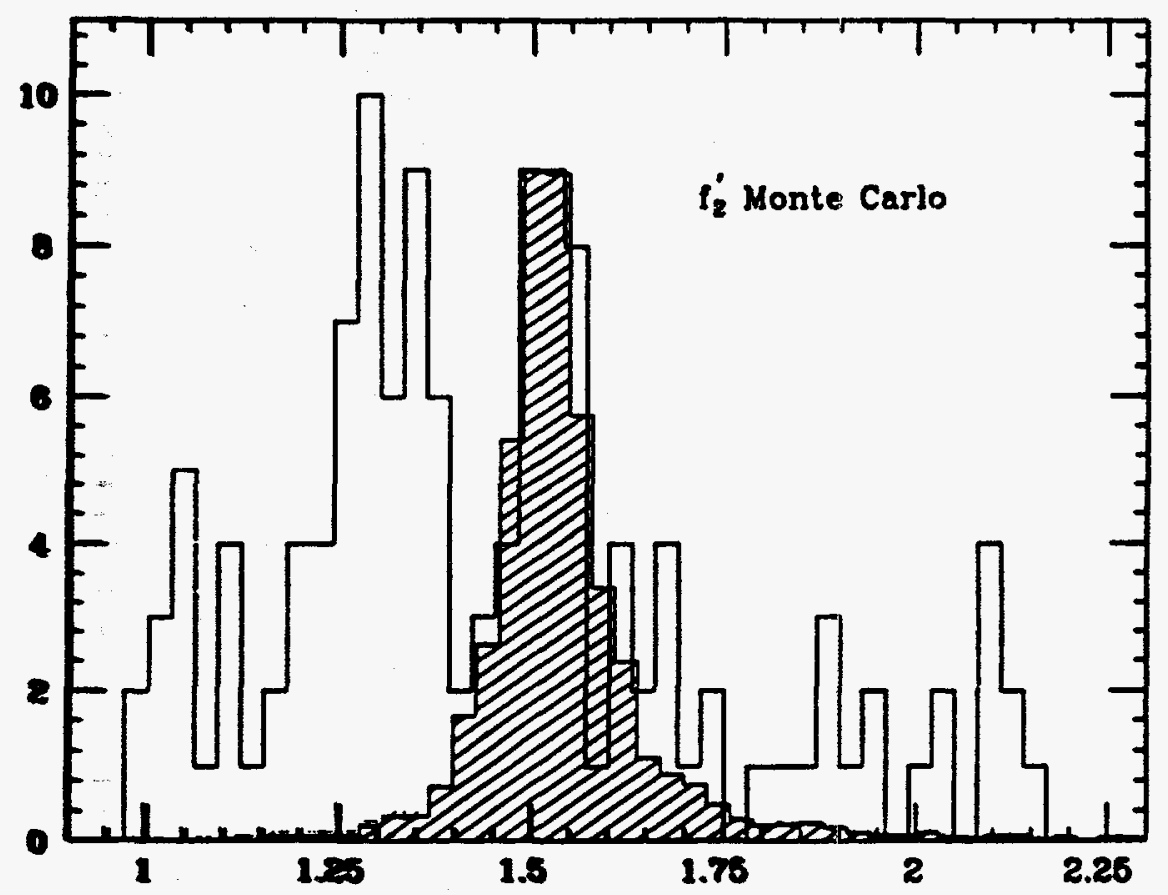

Figure 2.2: $\mathrm{K}^{+} \mathrm{K}^{-}$mass. The unshaded histogram is data, the shaded one M.C. 
Table 2.2: Properties of the tensor mesons $f_{2}(1270), a_{2}^{0}(1320)$, and $f_{2}^{\prime}(1525)$

\begin{tabular}{|c|c|c|c|c|}
\hline Particle & $\begin{array}{c}\text { Mass } \\
(\mathrm{MeV})\end{array}$ & $\begin{array}{c}\text { Width } \\
(\mathrm{MeV})\end{array}$ & $\begin{array}{c}\Gamma_{r \gamma} \\
(\mathrm{keV})\end{array}$ & $\begin{array}{c}\text { BR } \\
(\%)\end{array}$ \\
\hline$f_{2}(1270)$ & 1274 & 185 & 2.76 & 4.2 \\
& \pm 5 & \pm 20 & \pm 0.14 & \\
\hline$a_{2}^{0}(1320)$ & 1318 & 110 & 0.90 & 4.9 \\
& \pm 5 & \pm 5 & \pm 0.11 & \\
\hline$f_{2}^{\prime}(1525)$ & 1525 & 76 & 0.12 & $\sim 100$ \\
& \pm 5 & \pm 10 & \pm 0.04 & \\
\hline
\end{tabular}

After cutting out the $f_{2}^{\prime}$ signal and subtracting the background underneath it, we plot in Figure 2.3 the angular distribution $\cos \Theta^{*}$, which represents the decay angle of one of the kaons with respect to the $\gamma \gamma$ axis in the $\gamma \gamma$ center of mass. The solid curve in Figure.2.3 corresponds to the Monte Carlo expectation from a pure $f_{2}^{\prime}$ with helicity 2 , whereas the histogram represents the expectation from a helicity-0 $f_{2}^{\prime}$ Monte Carlo. Clearly, the data favor the helicity 2 hypothesis.

In general, the $Q^{2}$ dependence of the $\gamma \gamma$-width for a tensor meson produced in a singly-tagged two-photon reaction is given by three form factors, each associated with the three possible helicities 0,1 , and 2 . One has the following expression:

$$
\begin{aligned}
\Gamma_{\gamma \gamma^{*}}\left(Q_{1}^{2}, 0, W^{2}\right)=a \Gamma_{\gamma \gamma}^{\lambda=2} F^{(\lambda=2)}\left(Q_{1}^{2}, 0, W^{2}\right)^{2} & +b \Gamma_{\gamma \gamma}^{\lambda=0} F^{(\lambda=0)}\left(Q_{1}^{2}, 0, W^{2}\right)^{2} \\
& +c \tilde{\Gamma}_{\gamma \gamma^{*}}^{(\lambda=1)} F^{(\lambda=1)}\left(Q_{1}^{2}, 0, W^{2}\right)^{2}
\end{aligned}
$$

The important thing to mention here is that only if the helicity structure for a given tensor meson is known can one measure any of the above form factors. Here, since we have determined that the $f_{2}^{\prime}(1525)$ helicity is equal to 2 , only one term in the previous expression contributes, the one containing the form factor $F^{(\lambda=2)}$ which can then be measured. In Figure $2.4 \Gamma_{\gamma \gamma} \times F^{(\lambda=2)}$ is plotted in three bins of $Q^{2}$. The solid curve corresponds to a fit to the data using a $\phi$-pole form factor (in the fit, the expected value is determined using the area under the curve along each bin, thus ignoring the position of the data point in the bin) while the dashed curve is obtained by performing the same fit using a $\rho$-pole form factor. The extrapolation to $Q^{2}=0$ gives a measurement of the $\gamma \gamma$-width $\Gamma_{\gamma \gamma}$. Since the $f_{2}^{\prime}$ is widely believed to be made mainly of $s \bar{s}$, it is no surprise that the $\phi$ form factor seems to fit the data better than the $\rho$. Hence, we extract a value of

$$
\Gamma_{r_{1}}^{f_{2}^{\prime}} \times B R\left(f_{2}^{\prime} \rightarrow K \tilde{K}\right)=0.15 \pm 0.03(\text { stat. }) \pm 0.04(\text { syst. }) \mathrm{keV} \text {. }
$$




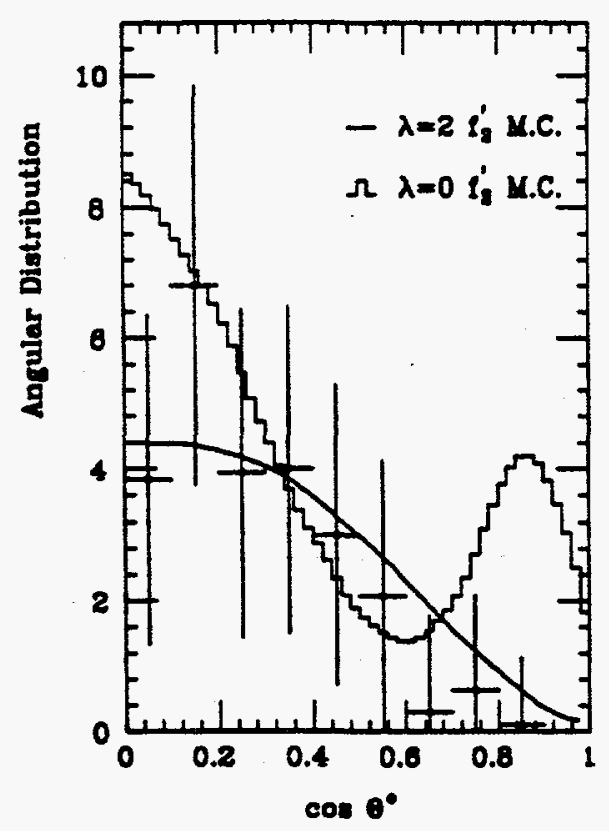

Figure 2.3: Center-of-mass angular distribution. The solid curve is for a $\lambda=2$ M.C. and the histogram a $\lambda=0$ M.C.

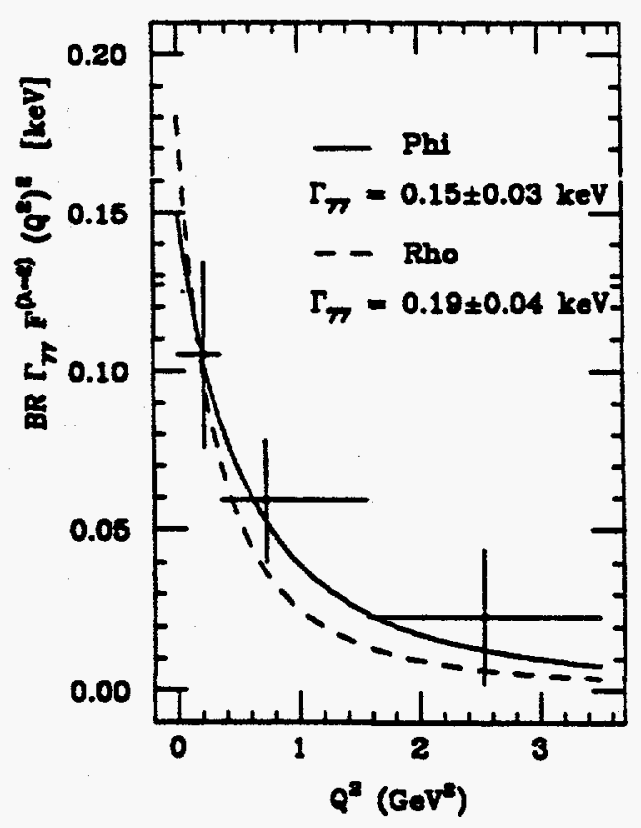

Figure 2.4: Form factor $F^{(\lambda=2)} \times \Gamma_{\eta r}$ vs. $Q^{2}$. The curves are fits using a $\phi$ (solid) and $\rho$ (dashed) form factors. 


\subsubsection{Future Projections}

The TPC $/ 2 \gamma$ physics program at high luminosity[12] is aimed at accumulating 1 $2 \mathrm{fb}^{-1}$ of data over a period of $2-3$ years and producing very competitive if not unique results in four areas of physics that are briefly described below. In order to achieve a luminosity of $10^{32} \mathrm{~cm}^{-2} \mathrm{sec}^{-1}$, further improvements to the storage ring PEP must be done. A minimum of three fills per day is required, and $\beta_{y}^{*}$ should be decreased to the design value of $4 \mathrm{~cm}$. In addition, preliminary tests have shown that a multi-bunching ( 9 bunches of $e^{-}$and 9 bunches of $e^{+}$) operation can be successfully implemented and in a timely manner; this would result in an increase in the luminosity by a factor of 3.

\section{Fragmentation}

The PEP energy range is well suited for the study of quark and gluon fragmentation processes. The energy is high enough for one to rise above the perturbative regime of QCD and be able to investigate the fragmentation of partons to hadrons, yet it is low enough to observe well defined two- and three-jet events containing all quark species. The particle identification of the TPC presents a great advantage. A data set of $1 \mathrm{fb}^{-1}$ would be required to produce definite results on the b-quark fragmentation; however a sample as low as $250-300 \mathrm{pb}^{-1}$ could yield interesting measurements of charm quark fragmentation.

\section{$\tau$ lepton physics}

The relative strength of $\tau$ production vs. hadron production, the hadronic vs. $\tau$ decay multiplicity, and the $\tau$ production kinematics make PEP the cleanest source of $\tau \bar{\tau}$ pairs available today. With $1 \mathrm{fb}^{-1}$ of data, one can perform a precise measurement of the $\tau$ lepton lifetime as well as a definitive study of $\tau$ decay systematics ("one prong puzzle"). The direct measurement (vertex method) of the $\tau$ lifetime $\tau_{\tau}$ can provide a check of the branching ratio $B_{e}$ for the decay $\tau \rightarrow e^{-} \bar{\nu}_{e} \nu_{\tau}\left(B_{e}\right.$ and $\tau_{\tau}$ are related by $\left.\tau_{\tau}=\left(m_{\mu} / m_{\tau}\right)^{5} \tau_{\mu} B_{e}\right)$, which in turn may result in a solution to the one prong puzzle. Furthermore a measurement of the ratio $R_{H}=\Gamma(\tau \rightarrow$ $\nu_{\tau}+$ hadrons $) / \Gamma\left(\tau \rightarrow e^{-} \bar{\nu}_{e} \nu_{\tau}\right)$ provides a check of QCD.

\section{B meson physics}

By running at the $\Upsilon(4 S)$ resonance, CESR is taking a major lead in this area. However, the B mesons produced at CESR are essentially at rest, whereas at PEP they are highly boosted; thus, PEP can provide additional and complementary results to those obtained at Cornell. In particular, valuable contributions can be made to the measurement of the $B$ meson lifetimes and to the measurements of mixing in the $B_{d}$ and $B_{s}$ systems. On the other hand, PEP may not be able to 
compete (in the near future) with LEP where large data samples are already being accumulated. Plans to further increase the PEP luminosity by an additional factor of two are being considered. They consist of multi-bunching as mentioned above, and a further reduction in $\beta_{y}^{*}$. The use of silicon pixel devices, which are already being developed at SLAC, mounted on a smaller radius $(<3.0 \mathrm{~cm})$ beam pipe will provide an increase in tagging efficiency.

\section{Two-photon physics}

Most of the work in untagged $\gamma \gamma$ physics has already been done, so the future in this field clearly lies in the detection of the $\operatorname{tag}(\mathrm{s})$. Here the TPC/2 $\gamma$ 's high-resolution low-angle tagging capability, possessed by none of the detectors at LEP, TRISTAN, or CESR, will allow it to play a leading role. With $1 \mathrm{fb}^{-1}$ of data, various tests on QCD will be performed; they include the quasi-real and virtual photon structure function measurements, jet production, inclusive charm production, and the formation of hadron pairs at high $p_{T}$. In addition, form factor measurements for pseudo-scalar and tensor mesons as well as a search for higher mass (>1.6 GeV) resonances and exotic states such as glueballs and hybrids will be carried out. Finally, a large data set will help understand the origin of the spin-1 resonance $\mathrm{X}$ (1420) (discovered by TPC $/ 2 \gamma$ ) and may reveal the existence of other such resonances. While two-photon physics at LEP or CESR is only a sidelight, it is a major part of the $\mathrm{TPC} / 2 \gamma$ physics program.

\subsubsection{Personnel}

W. Lin received his $\mathrm{Ph} . \mathrm{D}$. in the Spring of 1990 and has taken an Associate Professor position at National Central University in Taiwan. M. Daoudi will receive his Ph.D. in the Fall of 1990. A total of five graduate students will have completed their Ph.D. research on the TPC $/ 2 \gamma$ experiment. J. Layter and B. Shen will continue to participate in the TPC/ $2 \gamma$ experiment for UCR in a limited capacity. We will be responsible for the operation of the outer drift chamber and will share in the data taking and physics analysis. If a significant amount of data is accumulated, another graduate student may be assigned to this experiment.

\subsubsection{Conclusion}

At a time when a large fraction of the manpower in particle physics has shifted towards the high-energy machines like LEP, SLC, and the Tevatron collider, a lot remains to be done at the $15-30 \mathrm{GeV}$ range. The TPC/2 $\gamma$ detector at PEP is powerful and well- understood, with software and manpower already in place. In order for it to be competitive, however, it is crucial that its physics program be carried out in a timely manner. 


\subsection{OPAL at LEP}

\subsubsection{LEP Performance}

In 1989 the performance of LEP exceeded all expectations. On July 14, 1989, a highly symbolic date and only days after commissioning of the ring had begun, LEP machine physicists succeeded in maintaining a circulating beam in the ring. After a few weeks of optimization and tuning, a short pilot run began. The OPAL detector recorded LEP's first $Z^{0}$ event shortly before midnight on August 13 , and the LEP experiments were able to report their initial observations of events at the EPS meeting in Madrid at the beginning of September. The physics run which occupied the rest of the year covered energies between 88 and $95 \mathrm{GeV}$ in a scan around the $Z^{0}$ mass. OPAL collected a data sample of about $1.38 \mathrm{pb}^{-1}$ or nearly $30000 Z^{0}$ s.

In $1990 \mathrm{LEP}$ was supposed to produce a million $Z^{0} \mathrm{~s}$. No one doubted that this would be accomplished, and much effort was expended in crash programs to upgrade OPAL's event reconstruction capability and expand its direct access data storage capacity to keep pace with the expected avalanche of data. Unfortunately, the linear increase in luminosity observed at the end of last year has not been maintained into the new year, nor has the operating efficiency stayed at last year's levels. LEP has been running at rates between 10 and 20 times lower than the $0.5 \mathrm{~Hz}$ of $Z^{0}$ taken as the basis for the original projections. The peak luminosity attained so far, $5.5 \times 10^{30} \mathrm{~cm}^{-2} \mathrm{sec}^{-1}$, is a factor of 3 below expectation, and even that is rarely seen.

LEP machine physicists cite beam-beam limitations as the fundamental problem which is keeping the luminosity down: more current in the bunches does not lead to a corresponding increase in luminosity. Among the processes being investigated is quadrupole damping, in which off-axis $e^{ \pm}$can radiate in the fields of quadrupole magnets, limiting the effective aperture and reducing the accuracy of dispersion measurements. Other effects transfer horizontal dispersion into vertical dispersion, giving a direct reduction in luminosity. Setup times for running with new beam parameters are long during physics runs, but there is good reproducibility in machine development periods; this is a puzzle for the machine people. At the present time, no official luminosity projections are being circulated by LEP machine physicists. However, at the beginning of July, OPAL had collected some $66000 Z^{0} \mathrm{~s}$ or 2.843 $\mathrm{pb}^{-1}$. With no improvement in luminosity and small improvements in running efficiency of both the machine and the detector, the Collaboration should end the 1990 period with something close to a quarter of the hoped-for $Z^{0}$ s.

Another area of machine physics work is that of the absolute energy measurement which is proceeding on two fronts, both of which endeavor to find corrections relating the "best estimated energy" to the "field display energy" which determines the beam energy by evaluating the field integral using the field measurement given by a flip coil in a reference dipole in series with the ring magnets. The first line of attack 


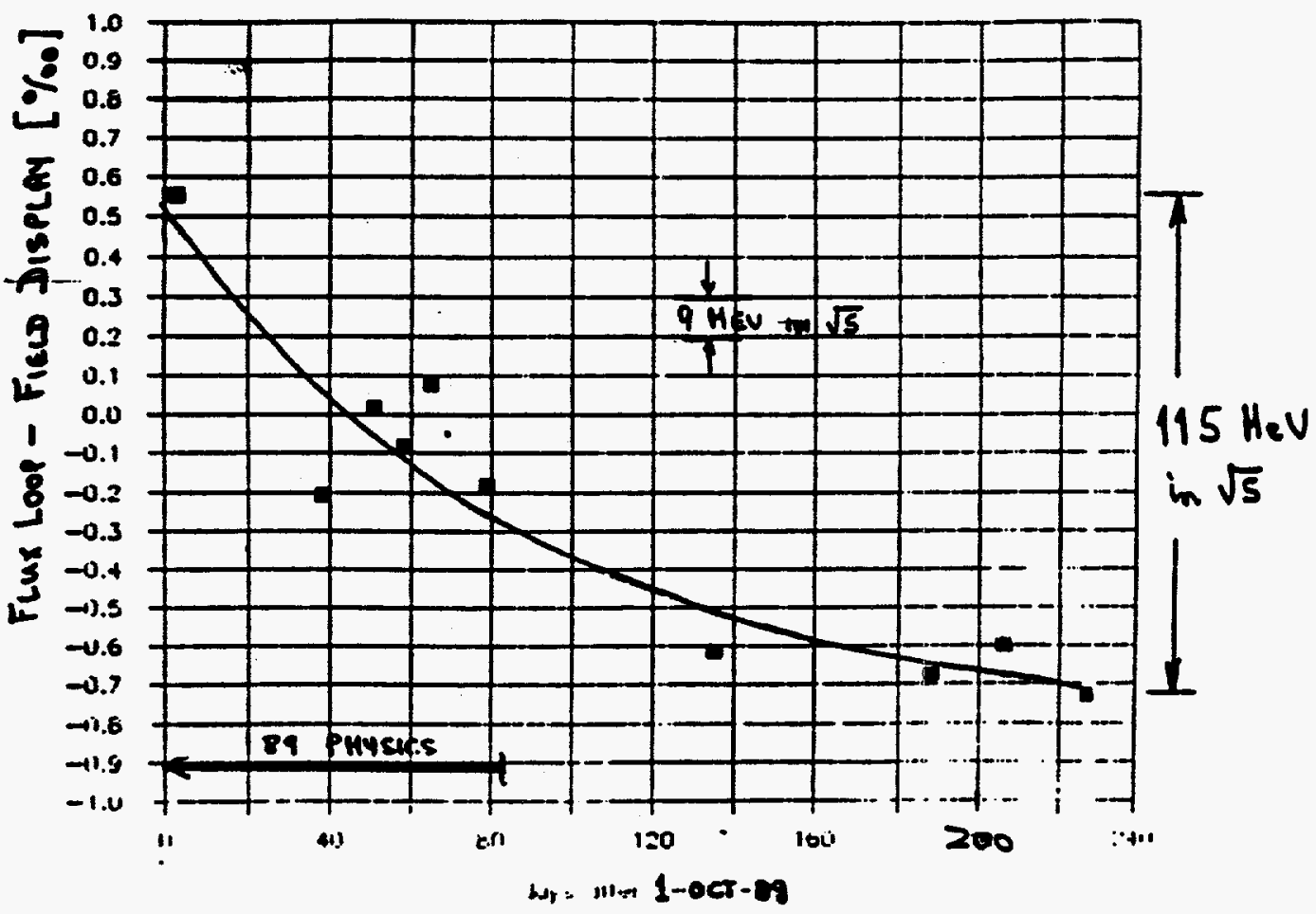

Figure 2.5: Per cent difference in field readings from the reference magnet and from a real LEP dipole as a function of time.

tries to find all relevant corrections, among them the magnet aging factors, offaxis corrections, effect of the earth's field, effect of nickel flashing on the vacuum chamber, etc. The "aging factor" arises from the fact that the "reference magnet" is different from all the ring magnets, being made entirely of iron, whereas the ring magnets are the famous LEP "concrete magnets," the laminations being spaced out along the tie rods and the spaces filled with concrete. The aging relative to the reference magnet is shown in Figure 2.5

The second method is the proton calibration method which attempts to measure the momentum of a proton beam on the same orbit as the electron beam by measuring the revolution frequency $f^{\text {rev }}$ for electrons and for protons. Then

$$
L=\frac{\beta_{e} c}{f_{e}^{r e v}}=\frac{\beta_{p} c}{f_{p}^{r e v}}
$$

where $L$ is the ring circumference. The quantity $f^{r e v}$ is related to the RF frequency through the harmonic number $h$

$$
f^{r e v}=f^{R F} / h
$$

One then solves for the proton $\beta$

$$
\beta_{p}=\beta_{e} \frac{f_{p}^{R F}}{f_{e}^{R F}} \frac{h_{e}}{h_{p}}
$$

to obtain the momentum of either beam. The weakness of this method is that LEP is not designed to accelerate protons, so the measurement must be made at injection 
energy, $20 \mathrm{GeV}$, and extrapolated to the vicinity of $45 \mathrm{GeV}$. The largest error is the error of the extrapolation. Both of these methods give corrections to the nominal energy on the order of $65 \mathrm{MeV}$, with a residual uncertainty of $21 \mathrm{MeV}$.

Recently the LEP machine group have reported the results of simulation studies that indicate the polarization yield might be considerably lower than anticipated. They pointed out however that even a low level of polarization, on the order of 3 to $5 \%$, is sufficient to permit energy calibration by resonance depolarization, a method which is extremely accurate and which does not suffer from the principal drawback of the proton method mentioned above.

Methods of increasing LEP luminosity beyond the design figure involve increasing the number of bunches. Currently each beam contains four bunches. The only scheme being actively considered is the 8 on 8 scenario which involves the installation of separators at the odd-numbered points. This could be done in two years at a cost of "only" 28 million Swiss francs, exclusive of necessary modifications to the detectors. However implementation of this scheme is "not considered urgent" at the present time. Higher numbers of bunches, the so-called "pretzel" schemes, reaching as high as 36 on 36 , involve running the beam off axis through the focussing magnets. In the light of the discussion of luminosity limitation above, these schemes are considered less feasible now than they were when first proposed. Necessary detector modifications become very costly as the interbunch time goes below $10 \mu$ secs.

\subsubsection{The OPAL Detector}

Last year's proposal included a comprehensive description of all the elements of the OPAL detector; and a definitive article describing the detector is nearly ready for submission to Nuclear Instruments and Methods after several years of preparation. Consequently we will limit the discussion here to a few salient points:

- Central Jet Chamber performance and problems.

- Improvements in the Forward Detector leading to reduced luminosity systematics.

- Hadron Calorimeter Strip Readout Hardware

- The Muon Chambers

- Data Acquisition and Filtering

\section{The Central Jet Chamber}

The Central Jet Chamber is the principal tracking chamber of OPAL. It not only provides the primary momentum measurement of the tracks and the means of event 


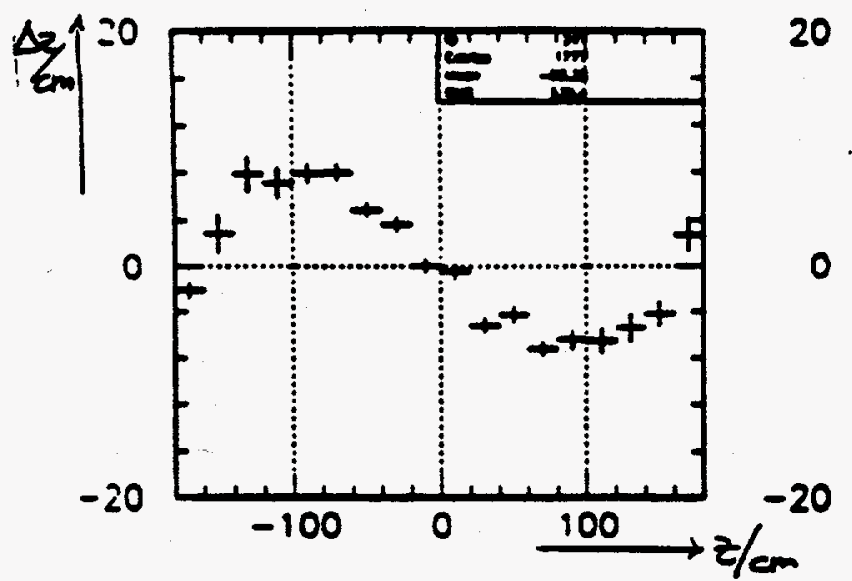

DZ.vs.Z, $-0.30<d y d x<-0.05$

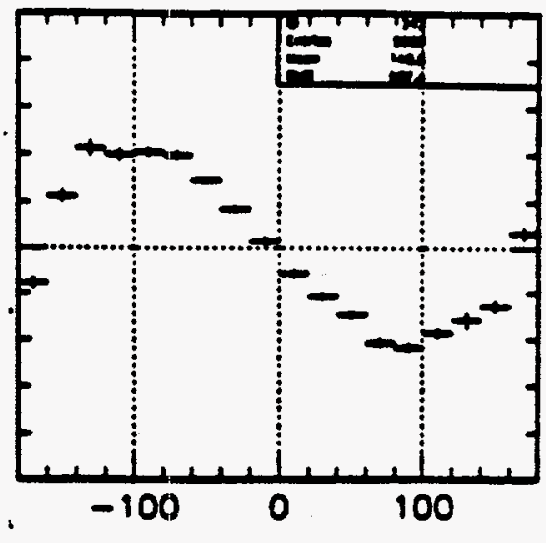

DZ. $s . Z,-0.05<d y d x<0.03$

Figure 2.6: Variations in $\Delta z$ as functions of $z$ and $\phi$

visualization, but also it is a key element in particle identification through its measurement of specific ionization. Although reconstruction of tracks in the $r-\phi$ plane has been adequate from the time of the Pilot Run in 1989, reconstruction in the r-z plane was severely lacking initially. Great improvement was made near the end of the 1989 run by finding and fixing noise sources, but z-resolution was still far from the design value, and this led to failures in track association with the outer detectors in many cases.

After the long shutdown at the end of the year, an order of magnitude improvement was made in restoring missing channels and repairing channels running at reduced efficiency. Recently some progress has been made in solving the $\mathrm{z}$ problem by abandoning efforts to find the root cause of the problem and by instead attempting to parametrize the necessary corrections to the $\mathrm{z}$ coordinate as functions of theta and phi. Of significant help in this endeavor has been the "dense data" package developed single-handedly by Keith Riles of the UCR group. This provides a packed version of the raw data as part of an enlarged DST and thus makes it possible to accumulate sufficient statistics to be able to map out the deviations as functions of likely variables. Some examples of this are shown in Figure 2.6

Another advance in the analysis has been a more accurate treatment of tail subtraction from the flash ADC data. Since jet chamber wires can in principle contain signals from several tracks, subsequent pulses must be corrected for the contributions of the tails of previous pulses. A better algorithm for performing this correction now exists and contributes to improved resolutions. At the present time, for identified muon pairs, the distance of closest approach in the $r-\phi$ plane has been brought down to 225 microns, and in the r-z plane to under $3 \mathrm{~cm}$. The momentum resolution is $12 \%$ at $45 \mathrm{GeV} / \mathrm{c}$ compared to the design resolution of $8 \%$. For muon pairs a clear separation of the sign of the charge can now be achieved, as shown on 

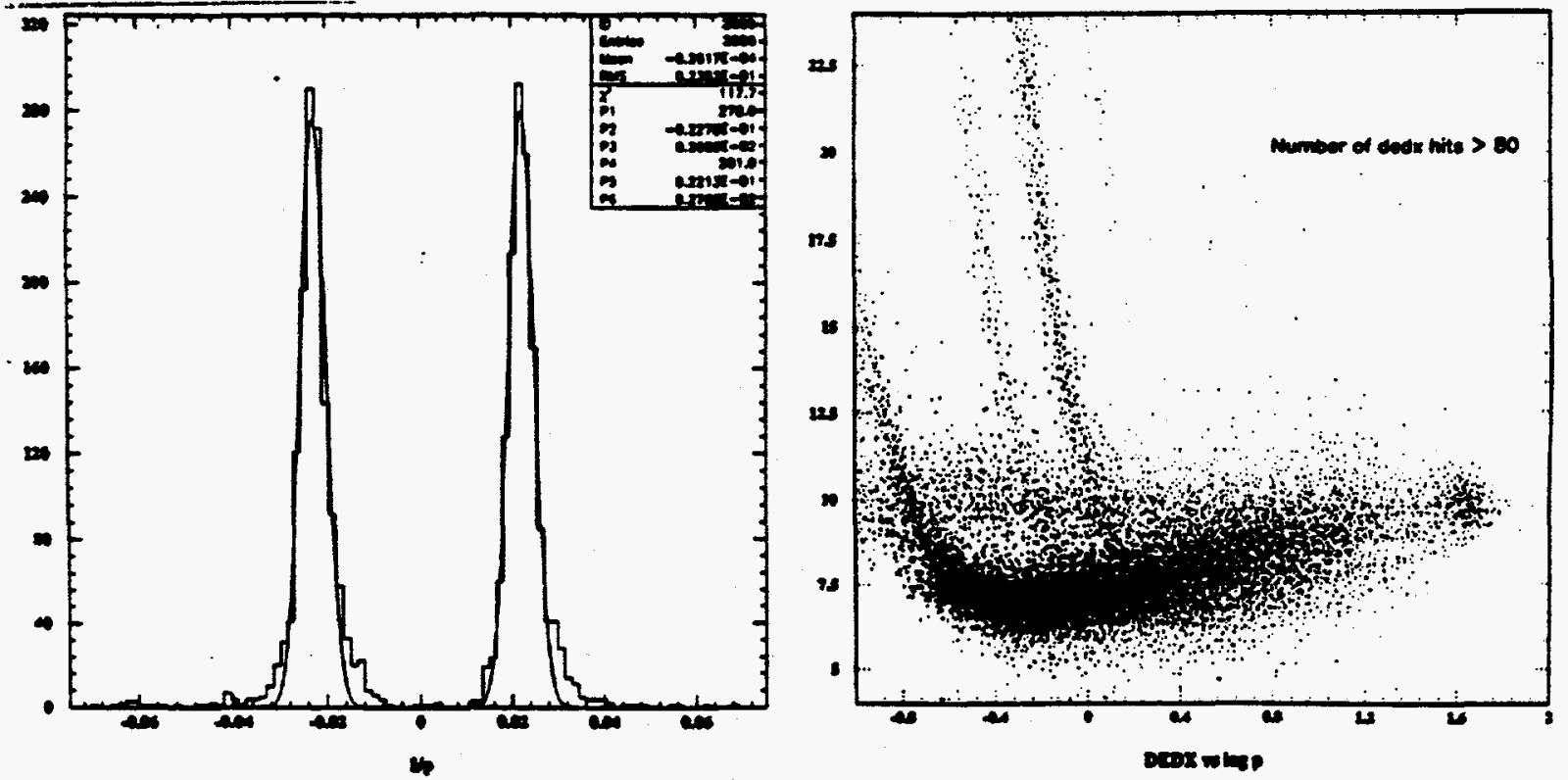

Figure 2.7: left Distribution of the signed value of $1 / p$ for muon pairs. right $d E / d x$ vs $\log p$

the left in Figure 2.7 The resolution in $\mathrm{dE} / \mathrm{dx}$ is now about $5 \%$ for tracks with more than 80 wires hit. The distribution ionization versus the log of momentum is shown in the same figure.

\section{The Forward Detector}

The Forward Detector is responsible for the calculation of OPAL luminosity. For the $1989 \mathrm{data}$, the systematic error in the luminosity evaluation was set at $2.1 \%$, dominated by a survey uncertainty of $1.5 \%$. Recently, the uncertainty has been reduced to below $1 \%$ by making use of the information from drift chambers located in front of the tube chambers in the calorimeter, as shown in Figure 2.8. This fixes the position of the tube chambers to a level of a few hundred microns. Further refinement of the tube chamber analysis package eliminates the effect of tracks which shower in the support material of the detector and give spurious tube chamber signals. It is estimated that the overall error in the luminosity determination will be in the neighborhood of $1.6 \%$ for the 1990 data.

The addition of the completely independent information from the fine luminosity scintillator counters provides a check on the value obtained from the calorimetertube chamber package. The luminosity values of these two methods agree to better than $1 \%$. 


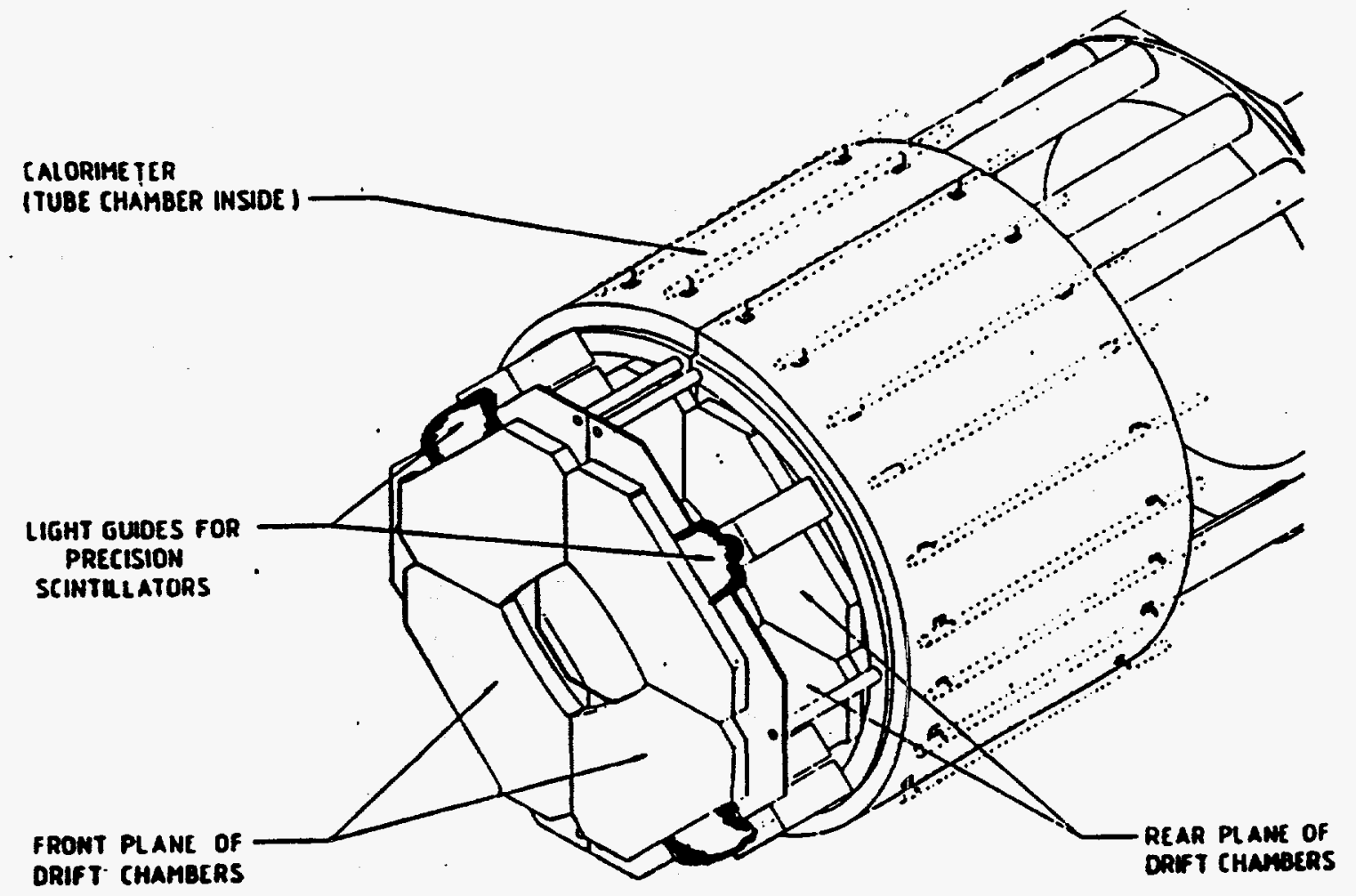

Figure 2.8: Perspective view of the Forward Detector

\section{Hadron Calorimeter Strip Readout}

During the past year of operation, the HCAL Strip Readout has performed at an acceptably trouble-free level. Five individual strip channels have died during that time (out of a total of close to 60,000). Maintenance has been necessary periodically however to repair repeated faults which occur always in the same location on the calorimeter and result in the temporary loss of several hundred strips. The cause of these faults remains a mystery, but improved online and offline monitoring provides quick feedback, and replacement of the affected units can be done rapidly.

Monitors for the power supplies to the front-end strip electronics have been installed, as well as temperature monitoring hardware and a wire chamber based gas quality monitor. Software monitoring tools which have been developed during this period include command procedures to check the integrity of the online data acquisition software permanent storage media, which have frequently become corrupted, and to back up these stored files. Both procedures run automatically once a day. In addition, an effort has been under way to exploit a number of available network tools, such as TCP/IP and the OPAL tool IMS, to improve our ability to do online monitoring.

During the long LEP shutdown starting this fall, it will be possible to access and repair all of the front-end electronics faults: the five dead strips which have occurred since the start of data-taking, along with the dozen or so known from before that start, plus a few cases of mysterious, but apparently benign, behavior. Hardware 
problems remaining to be investigated and solved include finding the source of the repeated front-end faults mentioned above, as well as the reason for an occasional (one in 2 or 3 thousand events) failure of a few of the 36 readout controller boards. In addition, these controllers must be studied to see what changes need to be made to them to make them compatible with future multi-bunch LEP running.

Repeated episodes of gas and high voltage problems indicate that more work needs to be done on the monitoring of the calorimeter gas. This will probably take the form of an installation of streamer tubes in the experimental pit to complement those on the surface, which are currently a very useful tool but are subject to temperature variation effects not seen below ground

\section{Muon Chambers}

The previous report stressed the originality of the "interpolating diamond" readout scheme for obtaining the $z$ coordinate from the barrel muon chambers. This method employs diamond shaped pads which pick up an induced signal of varying size depending on the position of the avalanche along the diamond. Sets of coarse and fine diamonds allow interpolation to achieve $1 \mathrm{~cm}$ resolution on a $10 \mathrm{~m}$ wire. Unfortunately in 1989 the resolution for the initial z determination from charge division on the wire was too poor to permit an unambiguous choice of the coarse diamond, so the entire scheme collapsed and the muon barrel detector provided only $\mathbf{r}-\phi$ information. Worse yet, the $\mathbf{r}-\phi$ information was frequently wrong because of errors in surveying. These two problems led to very low efficiency for merging muon track segments with tracks from the central detector.

In 1990 an extensive resurvey of the muon barrel chambers has eliminated nearly all the position errors, and significant improvement has been made in extracting the $z$ coordinate using the diamonds. At the present time nearly $90 \%$ of muon segments are successfully merged with central detector tracks. In addition, the installation of the muon endcap "patch" chambers above and below the beam line extends the coverage to about $96 \%$ of $4 \pi$.

\section{Data Acquisition and Filtering}

The OPAL data acquisition system incorporates distributed parallel processing in a VME bus structure to read out data from 16 separate detectors and assemble them into coherent events to be monitored, analyzed, and written onto output media. This system has been described in some detail previously, and, as in the case of the detector itself, a comprehensive review article is ready to be submitted for publication. Consequently, we will only itemize a few of the more important advances made during the past year.

- A persistent effort has been to reduce readout deadtime to a level of $10 \mathrm{msec}$ for each detector to permit the system to cope with the eventual increases in 


\section{luminosity.}

- Programming improvements have led to a 10-fold decrease in the response time of the Run Control program used to configure and steer the acquisition system. This results in much lower down time when detectors must be dropped from the readout because of local problems or added back in when the problems are solved.

- Improvements to the monitoring of each detector make it possible for the duty officer in charge of the experiment to spot detector malfunctions and initiate corrective action with minimal waste of beam time.

- The $370 / E$ emulators, designed to process events in real time, were judged to be obsolete before they were installed and were scrapped in favor of the commercial Apollo DN10000 multiprocessor system. Two of these devices, currently with a total of 6 processor boards, are able to keep up with present data rates. These produce DST output tapes containing the "dense data" discussed earlier, as well as the raw data. For 1991, it is planned to eliminate the raw data at this stage.

- The event filter has begun to run in active mode, that is, it rejects events judged to be junk instead of simply flagging them. This step was taken when scans of the junk events showed that they were in fact junk and no physics was being lost. At the same time, improvements to the trigger have resulted in only about $15 \%$ of triggered events being eliminated by the filter. 


\subsubsection{The $Z^{0}$ Line Shape from Hadronic Decays}

As the Standard Model confronted the LEP era, there emerged a new set of fundamental parameters: $\alpha, G_{F}$, and $m_{Z^{\circ}}$, which fixed to a large degree nearly all the calculable observables that one could envisage measuring. Of these variables, the one with the greatest uncertainty was $m_{Z^{0}}$, the uncertainty of which is listed as 1.8 $\mathrm{GeV} / \mathrm{c}^{2}$ in the latest Particle Data booklet. The measurement of the $Z^{0}$ mass was thus the principal goal of the four LEP experiments.

The mass measurement is obtained from a fit to the cross section for hadronic and leptonic decay modes as a function of energy as LEP scans over the region of the resonance. From the line shape which emerges from the scan, the total width of the $Z^{0}$ is obtained and the partial widths to hadrons and leptons can be determined. As a by-product, the "invisible width" can be deduced as the difference between the total width and the individual partial widths.

The LEP scan strategy was to dedicate half the luminosity in the vicinity of the $Z^{0}$ peak, already known roughly from the SLC measurement, and the remainder at three $1 \mathrm{GeV}$ intervals above and below the peak. In the 1989 running, the peak position was subdivided into three points $250 \mathrm{MeV}$ apart, but this was not repeated in 1990 running. At each machine energy, the multihadron cross section is calculated as

$$
\sigma\left(e^{+} e^{-} \rightarrow \text { hadrons }\right)=\frac{1}{\epsilon \int \mathcal{L} d t}\left(N_{\text {had }}-N_{b k g d}\right)
$$

where $\epsilon$ encompasses all selection and acceptance factors, and $\int \mathcal{L} d t$ is the integrated luminosity at the point in question. We summarize briefly the event selection criteria and the luminosity determination; details can be found in the published paper. [13]

The principal selection criteria for multihadronic events were based on the electromagnetic calorimeter since this detector element was the most stable and most carefully calibrated and was less dependent on beam conditions than the central detector jet chamber. Calorimeter hits are grouped into "clusters" which could involve only one lead glass block in the barrel, over a threshold of $100 \mathrm{MeV}$, or two adjacent blocks in the endcaps whose energy sums to at least $200 \mathrm{MeV}$. The requirements for multihadron candidates were then:

- at least 8 clusters, to eliminate decays to $e^{+} e^{-}$and $\tau^{+} \tau^{-}$,

- total energy deposited in the lead glass of at least $10 \%$ of the center of mass energy, to discard two-photon and beam-gas events,

- a limited energy imbalance along the beam direction, to reject beam-gas, beam-wall, and beam-halo events and endcap cosmic rays. Further cosmic ray rejection was provided by the TOF counters.

Backgrounds from various sources still present in the multihadron sample after all the selection requirements have been applied are estimated in several ways: from 
event scans, from Monte Carlo calculations, and from the data themselves by varying the cuts. Estimated contaminations are summarized below.

$\begin{array}{lc}\tau^{+} \tau^{-} & 0.33 \pm 0.04 \% \\ \text { two-photon (peak) } & 0.2 \pm 0.2 \% \\ e^{+} e^{-} & <0.1 \% \\ \text { cosmic } & <0.1 \% \\ \text { beam-wall } & <0.1 \% \\ \text { beam-gas } & <0.1 \% \\ \text { beam-halo } & <0.1 \%\end{array}$

Over fifty separate triggers are available in OPAL which makes it possible to use redundant triggers to determine the trigger efficiency. Comparing track-based and calorimeter-based triggers indicates that the inefficiency is $<0.01 \%$ in the barrel region $\left(\left|\cos \left(\theta_{T}\right)\right|<0.8\right.$, where $\theta_{T}$ is the direction of the thrust axis), and $<0.1 \%$ in the forward direction, $\left(\left|\cos \left(\theta_{T}\right)\right|>0.8\right)$. Detector acceptance has been calculated with the JETSET Monte Carlo and yields a result of $97.7 \pm 0.8 \%$. The primary sources of systematic error are uncertainties in the fragmentation model, imperfections in the detector simulation, the energy dependence in the region of the scan, and the error due to the specific choice of selection cuts.

The formalism for properly incorporating radiative corrections into the expressions for extracting the mass, width, and cross section from the uncorrected data had been developed carefully during the years prior to LEP turn-on. The observed cross section can be written as the convolution of the QED-corrected cross section $\tilde{\sigma}(s)$ with a radiator function $f\left(s, s^{\prime}\right)$

$$
\sigma(s)=\int_{m_{Z}^{2}}^{s} f\left(s, s^{\prime}\right) \tilde{\sigma}\left(s^{\prime}\right) d s^{\prime} .
$$

The cross section is expressed as a Breit-Wigner with an s-dependent width

$$
\tilde{\sigma}(s)=\sigma_{\text {had }}^{\text {pole }} \frac{s \Gamma_{Z}^{2}}{\left(s-m_{Z}^{2}\right)^{2}+s^{2} \Gamma_{Z}^{2} / m_{Z}^{2}}
$$

plus additional QED terms that contribute less than 1\%. The pole cross section

$$
\sigma_{\text {had }}^{\text {pole }}=\frac{12 \pi}{m_{Z}^{2}} \frac{\Gamma_{e} \Gamma_{f}}{\Gamma_{Z}^{2}}
$$

represents the resonance hadronic cross section at $s=m_{Z}^{2}$ without photonic corrections.

A "model-independent" fit to the scan data can be made to obtain the best values of $m_{Z}, \Gamma_{Z}$, and $\sigma_{h a d}^{\text {pole }}$. The results for the 1989 running, and for the combined totals from 1989 and 1990 are given in the following table: 

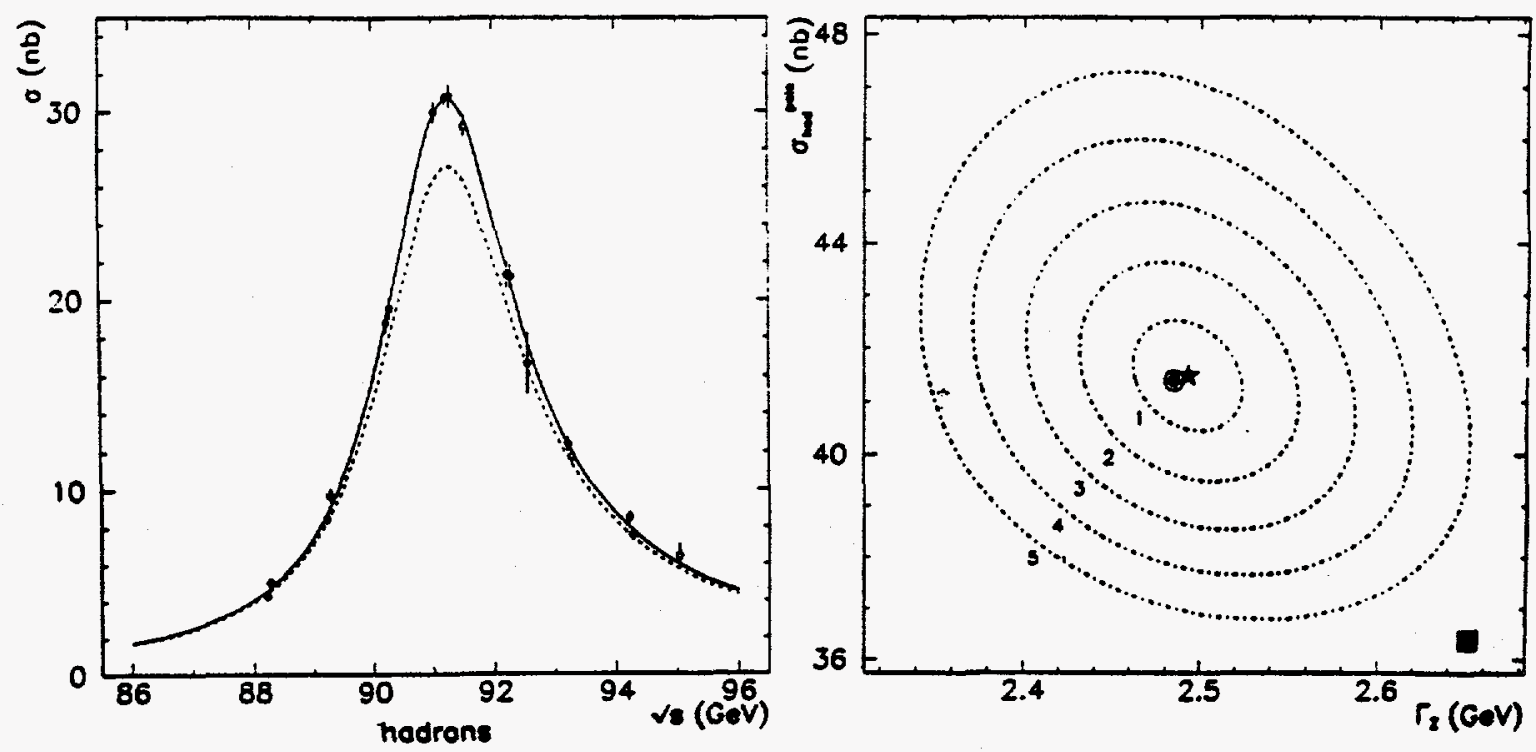

Figure 2.9: left Measured cross section for $e^{+} e^{-} \rightarrow$ hadrons. right Confidence contours in the $\sigma_{h a d}^{\text {pole }}$ vs $\Gamma_{Z}$ plane

\begin{tabular}{|l|c|c|}
\hline & 1989 & $1989+1990$ \\
\hline $\begin{array}{l}m_{Z} \\
(\mathrm{GeV})\end{array}$ & $91.145 \pm .022 \pm .027$ & $91.169 \pm .021 \pm .017$ \\
\hline$\Gamma_{Z}$ & $2.526 \pm .046$ & $2.493 \pm .031$ \\
$(\mathrm{GeV})$ & $41.2 \pm 1.1$ & $41.5 \pm 1.0$ \\
\hline $\begin{array}{l}\sigma_{\text {had }}^{\text {pol }} \\
(\mathrm{nb})\end{array}$ & $4.5 / 8$ & $10.9 / 15$ \\
\hline$\chi^{2} /$ dof & & \\
\hline
\end{tabular}

The first error given for the $\mathrm{Z}$ mass is statistical, the second accounts for the uncertainty in the LEP energy calibration. This part of the error quoted for the 1989 data includes an additional uncertainty in the energy calibration which is correlated for the 1989 and 1990 data sets. These results are shown in Figures 2.9 .. The left hand figure is the measured cross section for $e^{+} e^{-} \rightarrow$ hadrons. The solid curve is the line shape from the combined fit. The dashed curve is the predicted line shape from the Standard Model, assuming the measured $m_{z}$ and four neutrino generations. The right hand figure shows the confidence contours in the $\sigma_{\text {had }}^{\text {pole }}$ vs $\Gamma_{z}$ plane. The OPAL fit to the hadronic data is represented by the star, whereas the Standard Model prediction is given by the circle.

The number of light neutrinos, $N_{\nu}$, can be determined by a fit involving the parameters $m_{Z}$ and $N_{\nu}$, taking all other values from the Standard Model predictions. The $\mathrm{Z}$ width is expressed as

$$
\Gamma_{Z}=\Gamma_{\text {had }}^{S M}+3 \Gamma_{e}^{S M}+N_{\nu} \Gamma_{\nu}^{S M}
$$


where

$$
\Gamma_{\text {had }}^{S M}=1734_{-21}^{+47} \mathrm{MeV} \quad \Gamma_{e}^{S M}=83.4_{-0.6}^{+1.3} \mathrm{MeV} \quad \Gamma_{\nu}^{S M}=166.2_{-0.7}^{+2.7} \mathrm{MeV}
$$

The result is $N_{\nu}=3.02 \pm 0.15$. The error includes the theoretical uncertainty in the top quark mass, the Higgs mass, and the strong coupling constant. The dashed curve in the figure is the line shape predicted by the Standard Model assuming the measured value of the $\mathrm{Z}$ mass and four neutrino generations.

Members of the UCR group G. VanDalen and M. Dittmar organized the Working Group that obtained these results, and VanDalen made the first presentation of OPAL's numbers at the DPF meeting at Houston in January. 


\subsubsection{Leptonic Decays and the Combined Analysis}

Data samples have been obtained for the leptonic channels $e^{+} e^{-} \rightarrow e^{+} e^{-}, \mu^{+} \mu^{-} \rightarrow$ $\mu^{+} \mu^{-}$, and $\tau^{+} \tau^{-} \rightarrow \tau^{+} \tau^{-}$using criteria described below. In the analysis of the 1990 data, the angular acceptances have been extended to include more of the forward region than it was feasible to do in the case of the 1989 data.

\section{The $e^{+} e^{-}$Channel}

To select $e^{+} e^{-}$candidates, one requires two lead glass clusters in the angular range $|\cos \theta|<0.80$, each of which has at least $50 \%$ of the beam energy. The total energy reconstructed from the calorimeter information has to be at least $85 \%$ of the center of mass energy, and no more than 8 clusters of any energy could be present in the detector. An acollinearity cut between the detected leptons is made to reject events which have undetected hard photons escaping down the beam pipe. Cuts are made on $k$, the photon energy, and $\delta$, the opening angle between the photon and the final state lepton, to facilitate comparison with the analytic formulas used for the parametrization. To minimize $t$ channel effects, the cross section is evaluated over the asymmetric region $-0.70<\cos \theta_{e}-<0.40$.

Again using the many redundant OPAL triggers, one can estimate the trigger efficiency to be $99.9 \%$. The acceptance over the angular range mentioned above is calculated to be $99.1 \pm 0.7 \%$ using the BABAMC Monte Carlo program. Contamination from the $\tau^{+} \tau^{-}$channel is determined to be $0.1 \pm 0.1 \%$ using the KORALZ program, and background contributions from multihadrons and from two-photon processes are less than $0.1 \%$.

\section{The $\mu^{+} \mu^{-}$Channel}

Dimuon candidates are identified by requiring two tracks in the angular region $|\cos \theta|<0.82$ that satisfy certain general track quality cuts. Requiring a track momentum of $6 \mathrm{GeV} / \mathrm{c}$ suppresses $\tau^{+} \tau^{-}$contamination, and demanding that the acollinearity between the two tracks be less than $15^{\circ}$ reduces the contribution from two-photon processes.

Each of the two tracks must further be identified as a muon by one of the following criteria: i) 2 hits in either the barrel or endcap muon chambers that fall within 70 mrad of the projected extrapolated track; ii) at least 5 of a possible 9 hits in a similar road in the hadron calorimeter; iii) a momentum measured in the central detector of more than $15 \mathrm{GeV} / \mathrm{c}$ accompanied by a deposition in the calorimeter of less than 3 $\mathrm{GeV}$. Trigger efficiency is found to be $99.2 \pm 0.5 \%$ and the acceptance over the stated angular range is $75.0 \pm 1.0 \%$ as determined by the KORALZ program. Using the same program one finds the backgrounds from $\tau^{+} \tau^{-}$and dimuons from two-photon processes to be $3.7 \pm 0.9 \%$ and $0.1 \pm 0.1 \%$ respectively. 


\section{The $\tau^{+} \tau^{-}$Channel}

One selects tau candidates by asking that the number of charged tracks be between 2 and 6 . To suppress multihadron candidates, there must be fewer than 11 electromagnetic clusters. Dimuon pairs are removed by requiring that the summed electromagnetic energy be between 3 and $80 \%$ of the center of mass energy. Standard track quality cuts are imposed. Once these criteria are satisfied, one picks the taus out of the tracks by an iterative procedure which begins by defining a cone of $35^{\circ}$ half angle around the highest energy cluster, summing the momenta of all tracks in the cone, then defining a new direction. This process is repeated until there are no further particles in the vicinity, and the resulting agglomeration is considered a tau if it contains at least one charged particle with at least $1 \%$ of the beam energy. If two taus are found by the procedure, the event is a $\tau^{+} \tau^{-}$candidate if the acolinearity of the particles is less than $15^{\circ}$, and if they are within an angular acceptance of $|\cos \theta|<0.85$.

The by now familiar redundant trigger method gives a trigger efficiency of $99.8 \%$, and the acceptance in the angular region is $67.8 \pm 2.5 \%$. Background from multihadrons are studied with the JETSET Monte Carlo and found to be $0.9 \pm 0.7 \%$. Similarly contamination from $\mu^{+} \mu^{-}$pairs and from beam-gas are estimated at $0.7 \pm 0.4 \%$ and $0.4 \pm 0.3 \%$ respectively. The QED process $e^{+} e^{-}$contributes $1.1 \pm 0.8 \%$ and various two-photon processes add $0.4 \pm 0.4 \%$. A requirement of opposite sign eliminates $2.1 \%$ of the events.

\section{Fitting Formulae}

The fits are based on a $\chi^{2}$ minimization procedure which takes into account the full correlation matrix of the experimental uncertainties. The analysis is termed "combined" since the hadronic cross section data are included and $m_{Z}, \Gamma_{Z}$, and $\Gamma_{\text {had }}$ are treated as free parameters in the fit. Radiative corrections are treated with the ZBIZON program, although the prescriptions of Greco [14] and Caffo, et al [15] are applied in an ad hoc manner to the $e^{+} e^{-}$channel. If pure QED and $\gamma-Z$ inteference terms of order $1 \%$ are omitted, the expression used in the fit is of the form

$$
\frac{d \sigma}{d \cos \theta}\left(e^{+} e^{-} \rightarrow l^{+} l^{-}\right)=\left|\chi(s)^{2}\right|\left[C_{Z Z}^{(1)}\left(1+\cos ^{2} \theta\right)+C_{Z Z}^{(2)} \cos \theta\right]
$$

with

$$
\chi=\frac{\mathrm{G}_{F} m_{Z}^{2}}{8 \pi \alpha \sqrt{2}}\left(\frac{s}{s-m_{Z}^{2}+i s \Gamma_{Z} / m_{Z}}\right)
$$

The expression $C_{Z Z}^{(1)}$ can be written as

$$
C_{Z Z}^{(1)}=\left(\frac{24 \pi \sqrt{2}}{\mathrm{G}_{F} m_{Z}^{3}} \Gamma_{l+l-}\right)^{2}
$$



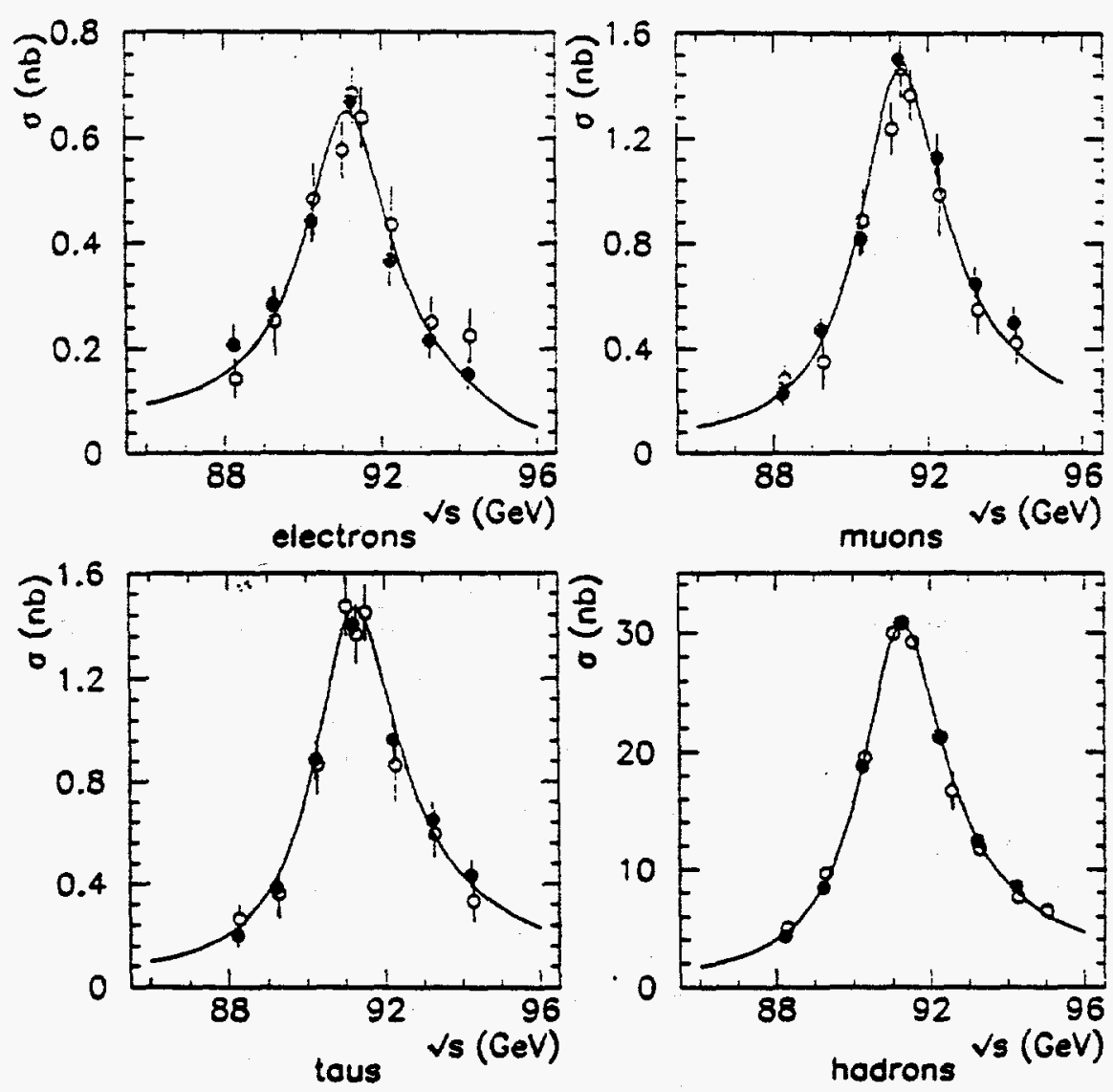

Figure 2.10: Measured cross sections of the various channels. The solid curves correspond to the combined fit.

while

$$
C_{Z Z}^{(2)}=8 \hat{a}_{l}^{2} \hat{v}_{l}^{2}
$$

\section{Results of the Fits}

The selection procedures described above yield $1800 e^{+} e^{-}, 3050 \mu^{+} \mu^{-}$, and 2550 $\tau^{+} \tau^{-}$events. The measured cross sections are shown in Figure 2.10 .. Fitting for the leptonic channels separately, one obtains

$$
\Gamma_{e^{+e^{-}}}=84.5 \pm 3.1 \quad \Gamma_{\mu^{+} \mu^{-}}=81.9 \pm 2.4 \quad \Gamma_{\tau^{+} \tau^{-}}=82.6 \pm 2.6
$$

where all values are in $\mathrm{MeV}$. On the basis of these results, one concludes that lepton universality is justified and can then make a global fit for all leptons obtaining $\Gamma_{1+l-}=83.1 \pm 1.9 \mathrm{MeV}$. The value of the $\mathrm{Z}$ mass obtained in this fit is $91.151 \pm 0.021$ 
$\mathrm{GeV}$ while $m_{Z}=2.527 \pm 0.044 \mathrm{GeV}$ and $\Gamma_{\text {had }}=1.804 \pm 0.044 \mathrm{GeV}$. These values are clearly in good agreement with the values obtained from the three parameter fit to the hadronic data alone. It should be pointed out that there is an additional error in the $\mathrm{Z}$ mass of $30 \mathrm{MeV}$ arising from the uncertainty of the beam energy. One can avoid the complications of the $t$ channel to the $e^{+} e^{-}$channel by dealing only with the $\mu^{+} \mu^{-}$and $\tau^{+} \tau^{-}$channels. If this is done, one obtains a value of $\Gamma_{l+l}$ of $82.2 \pm 2.1 \mathrm{MeV}$ while $m_{Z}=91.146 \pm 0.021 \mathrm{GeV}$. Consequently we conclude that no serious error is being made in the treatment of the electron channel.

Having obtained the individual widths from the foregoing analysis, we can procede to evaluate the "invisible" width, obtaining $\Gamma_{\text {inv }}=474 \pm 43 \mathrm{MeV}$, whence $N_{\nu}=2.85 \pm 0.26(\exp )_{-0.04}^{+0.02}$ (theor) for the number of light neutrinos. The ratio $R_{Z}=\Gamma_{\text {had }} / \Gamma_{l+l}$ is found to be $21.72_{-0.65}^{+0.71}$, which is within 1.5 standard deviations of the Standard Model prediction. The value of $\hat{a}_{l}^{2} \hat{v}_{l}^{2}$ obtained in the global fit is $0.0054 \pm 0.0046$, also in agreement with Standard Model predictions.

The measured forward-backward asymmetries are displayed in Figure 2.11 as functions of the center of mass energy. By including the terms involving the $\gamma-Z$ interference in the fit equation, one can obtain the values of $\hat{v}_{l}^{2}$ and $\hat{a}_{l}^{2}$ separately. Making the substitutions

$$
\hat{a}_{l}^{2} \rightarrow \rho_{Z}
$$

and

$$
\hat{v}_{l}^{2} \rightarrow \rho_{z}\left(1-4 \sin ^{2} \bar{\theta}_{W}\right)^{2}
$$

we find

$$
\rho_{Z}=0.998 \pm 0.024 \quad \text { and } \sin ^{2} \bar{\theta}_{W}=0.233_{-0.012}^{+0.045}
$$

A more precise value can be obtained by imposing Standard Model constraints between $\rho_{Z}$ and $\sin ^{2} \bar{\theta}_{W}$, with a minimal Higgs structure. The result is

$$
\sin ^{2} \bar{\theta}_{W}^{S M}=0.233_{-0.006}^{+0.007} \pm 0.002
$$

where the second error is theoretical. 

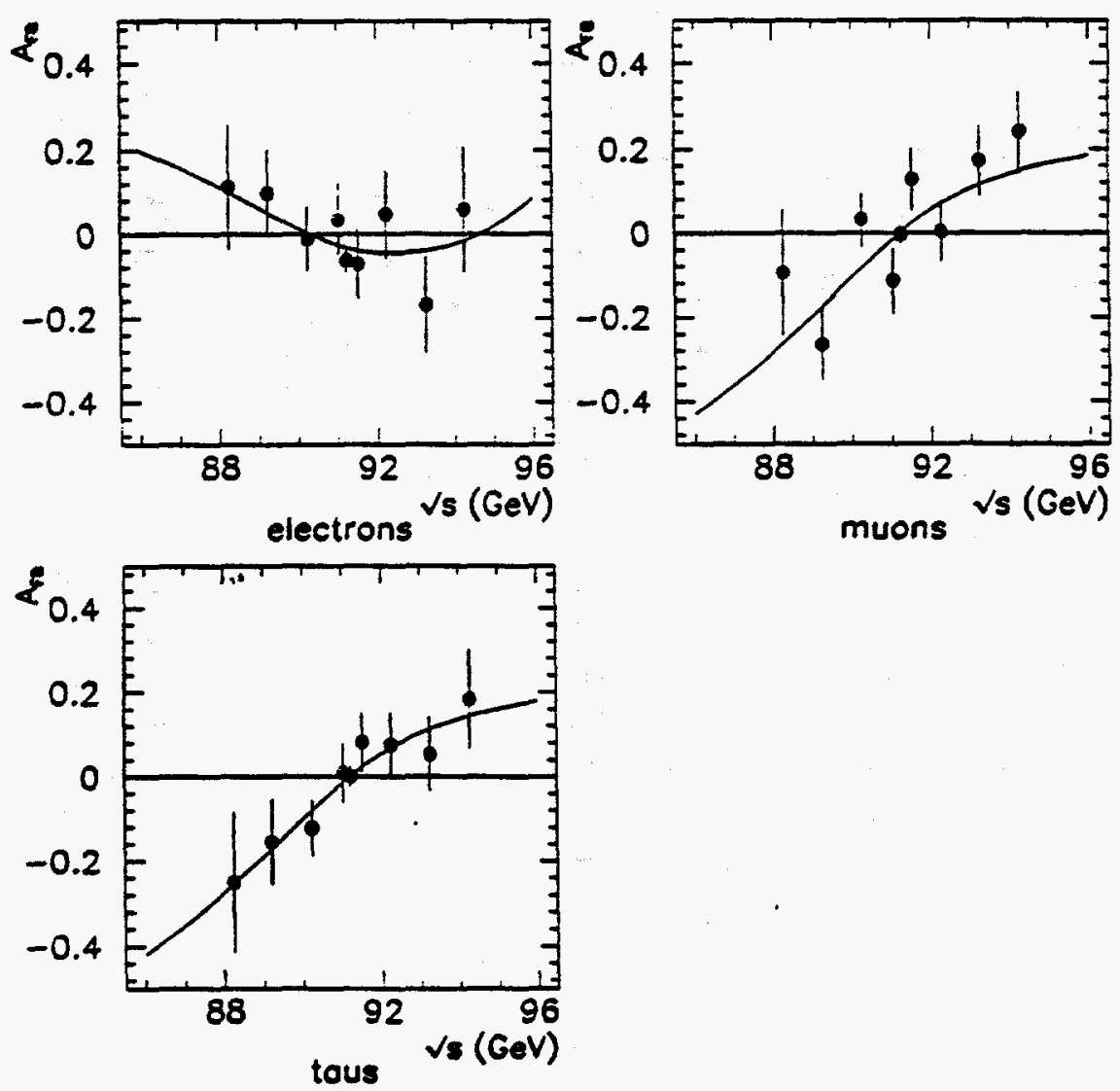

Figure 2.11: Measured forward-backward asymmetries of leptonic final states versus the center of mass energy. 


\subsubsection{Quantum Chromodynamics}

\section{Global Event Shape Analysis}

The measurement of distributions which characterize hadronic event structure allows a test of the consistency of Quantum Chromodynamics (QCD) in describing experimental data. Global event shape variables such as thrust and sphericity are sensitive to the distributions of quarks and gluons created in the $e^{+} e^{-}$annihilation process. Unfortunately there is no theoretical description for the mechanism of hadronization or fragmentation by which quarks and gluons become confined inside hadrons. Consequently, QCD calculations do not directly predict the distributions

of the observed hadrons. Instead, perturbation theory is incorporated into Monte Carlo simulation programs which are used to generate parton states, and models of the fragmentation process are applied to these partons to obtain the final hadron states which can be compared to the measured distributions.

A number of Monte Carlo programs describing hadronic production in $e^{+} e^{-}$ interactions have been developed. Each represents a particular approach in the application of perturbation theory to QCD and in the modeling of confinement. Four of the best known of these programs have been chosen for comparison: Jetset version 7.2 (the Lund model by Sjöstrand and collaborators), Herwig version 3.4 (by Marchesini and Webber), Ariadne version 3.1 (by Petterson et al), and the second order matrix element program known as ERT-E0 (produced by Magnussen and based on work of Ali and Barreiro and of Ellis, Ross, and Terrano). A discussion of each of the models and references to the original work are found in the OPAL published report. [16]

The event sample used in the study of event shapes is substantially the same as that already discussed in the section on line shape. Two separate analysis paths are followed, one based on electromagnetic clusters and the other on charged tracks, to verify the consistency of the measurements and to establish that they are free of systematic biases, due in particular to imperfections in the simulation of the detector.

A large number of event shape variables have been formulated to characterize the structure of hadron production in $e^{+} e^{-}$annihilation:

- Thrust $T$ defines the event axis $\hat{n}$ and is given by

$$
T=\max \left(\frac{\sum_{i}\left|\vec{p}_{i} \cdot \hat{n}\right|}{\sum_{i}\left|\vec{p}_{i}\right|}\right) .
$$

Its value is 1 for two jet events and 0.5 for isotropic events.

- Thrust major $T_{\text {major }}$ signals three-jet structure and is the same quantity evaluated in the plane perpendicular to the thrust axis. 
- Thrust minor $T_{m i n o r}$ is evaluated along the direction perpendicular to the two previous quantities and will be 0 for planar events.

- Oblateness $O$ is $T_{m a j o r}-T_{m i n o r}$ and is 0 for symmetric events or inflated footballs.

- Sphericity $S$ is $3\left(Q_{1}+Q_{2}\right) / 2$ and is 1 for spherical events

- Aplanarity $A$ is $3 Q_{1} / 2$ and is 0 for planar events. The $Q_{i}$ are the eigenvalues of the momentum tensor $S^{\alpha \beta}$ given by

$$
S^{\alpha \beta}=\frac{\sum_{i} p_{i}^{\alpha} \cdot p_{i}^{\beta}}{\sum_{i} p_{i}^{2}} \quad \alpha, \beta=1,2,3
$$

where $i$ runs over all the particles in an event.

- The $D$ variable is given by $D=27 \cdot\left(\lambda_{1} \cdot \lambda_{2} \cdot \lambda_{3}\right)$, where the $\lambda_{i}$ are eigenvalues of the spherocity tensor, the linear analog of the sphericity tensor. $D$ will be nonzero for four-jet events.

- The normalized 2nd Fox-Wolfram moment $H_{2} / H_{0}$ is $\approx 1$ for two-jet events but contains no reference to an axis as does Thrust.

- $\ln \left(1 / x_{p}\right)$ where $x_{p}=2\left|\vec{p}_{i}\right| / E_{c . m}$.

- The charged track multiplicity $n_{c h}$.

The strategy of the analysis has been to "tune" the parameters of the four Monte Carlo programs using only two of these variables: the normalized Fox-Wolfram moment and $T_{\text {major. }}$. The other quantities calculated from the tuned programs are then compared with the data. Jetset, Herwig, and Ariadne, all based on the parton shower approach, are in good agreement with the data. The ERT-E0 model provides a reasonable description of regions of distributions dominated by three-jet production, but it does not describe other regions well. Comparisons of OPAL data with the predictions for thrust and oblateness are shown in Figure 2.12. The distribution of charged multiplicity at the detector level, i.e., uncorrected for detector acceptance, is shown in Figure 2.13 together with the predictions of Jetset and Herwig. From this we obtain an unfolded value for the mean charged multiplicity

$$
n_{\text {ch }}=21.28 \pm 0.04 \text { (stat) } \pm 0.84 \text { (syst) }
$$

The aplanarity distribution of OPAL at $91 \mathrm{GeV}$ is shown in (b) of the same figure together with those of TASSO at $35 \mathrm{GeV}$ and of Mark II at $29 \mathrm{GeV}$, together with the Jetset predictions, which are seen to describe the data well for all energies. 

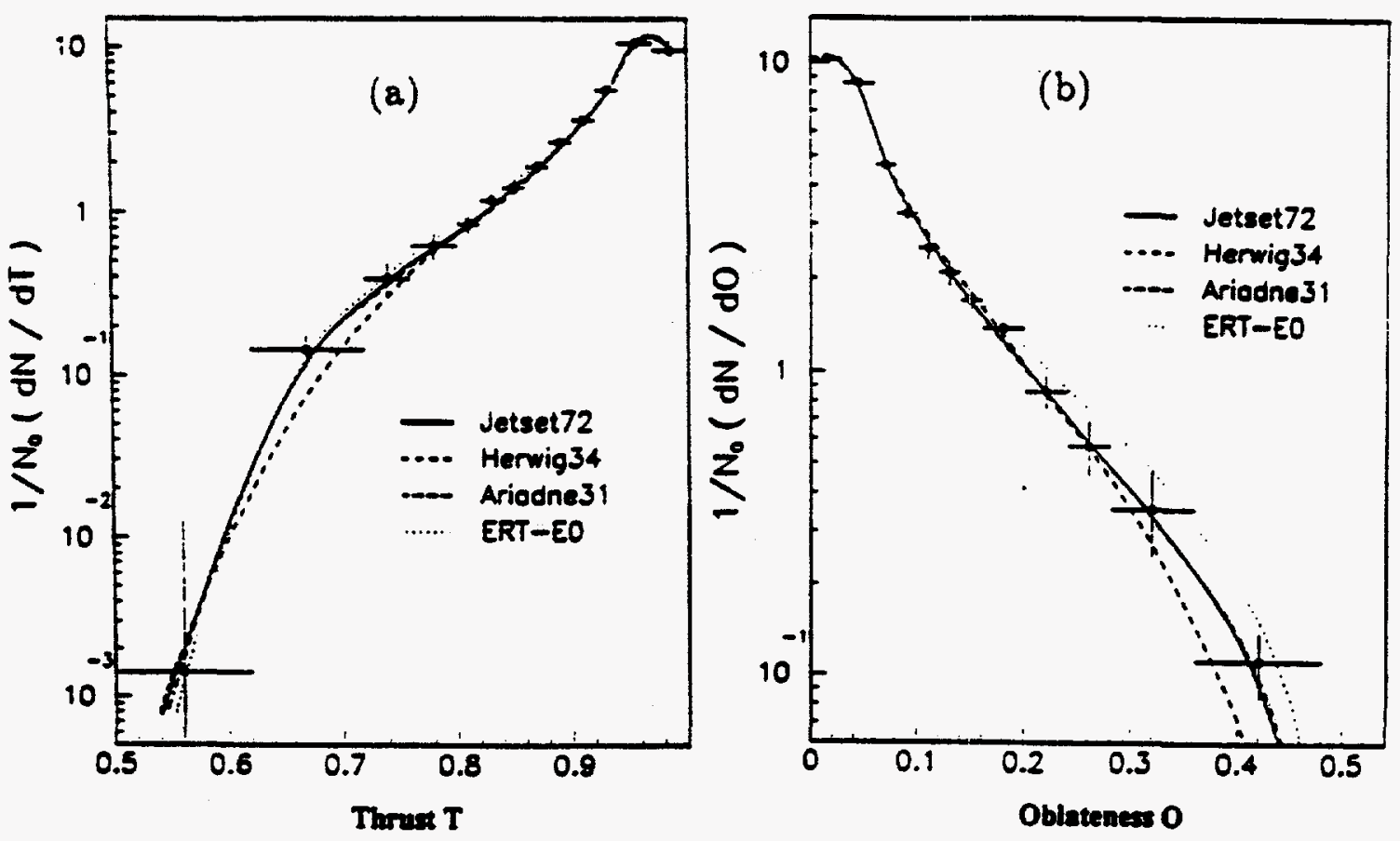

Figure 2.12: (a) Distribution of the Thrust variable, and (b) Distribution of the Oblateness variable
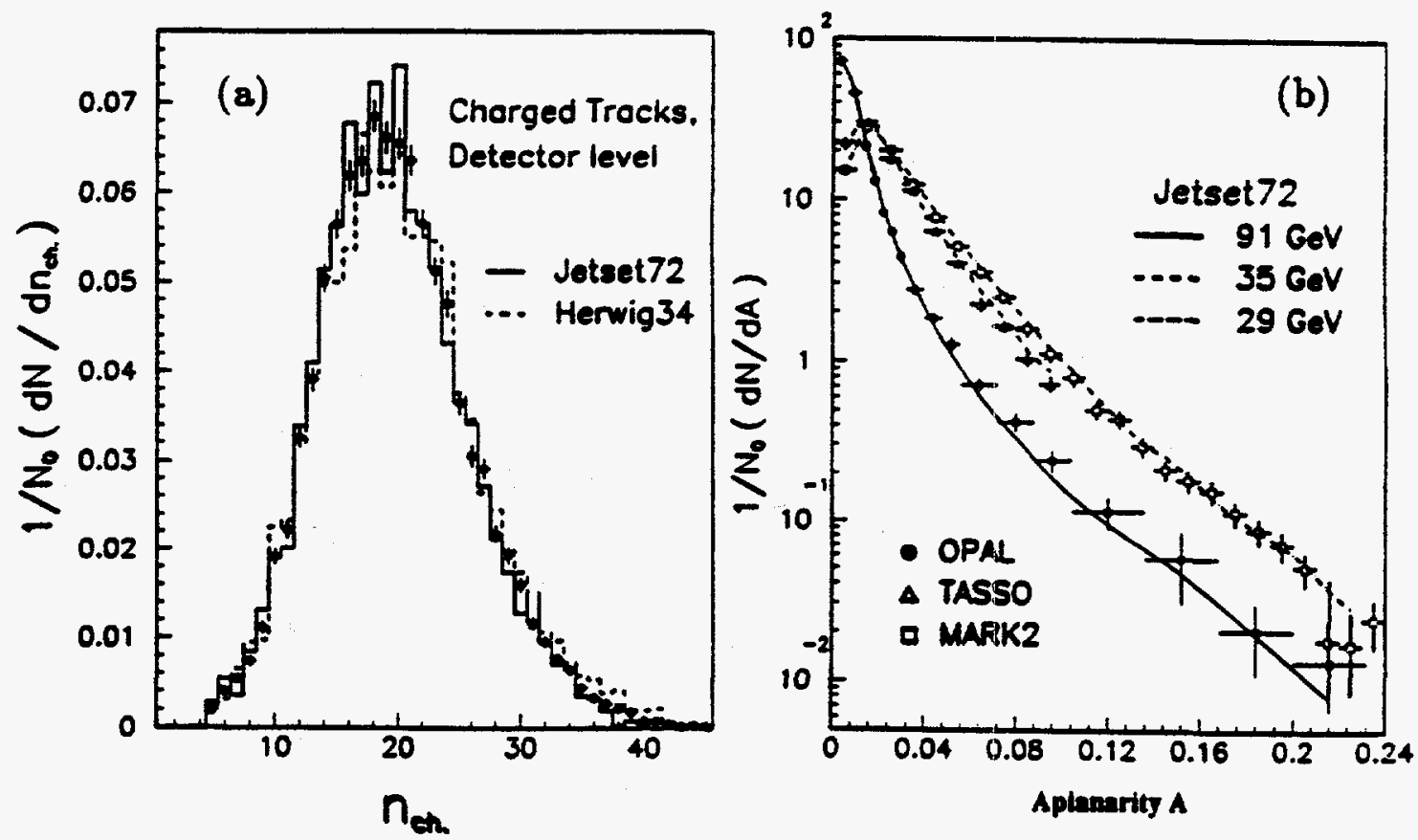

Figure 2.13: (a) Charged multiplicity at the detector level, and (b) Distribution of the aplanarity variable for several energies 

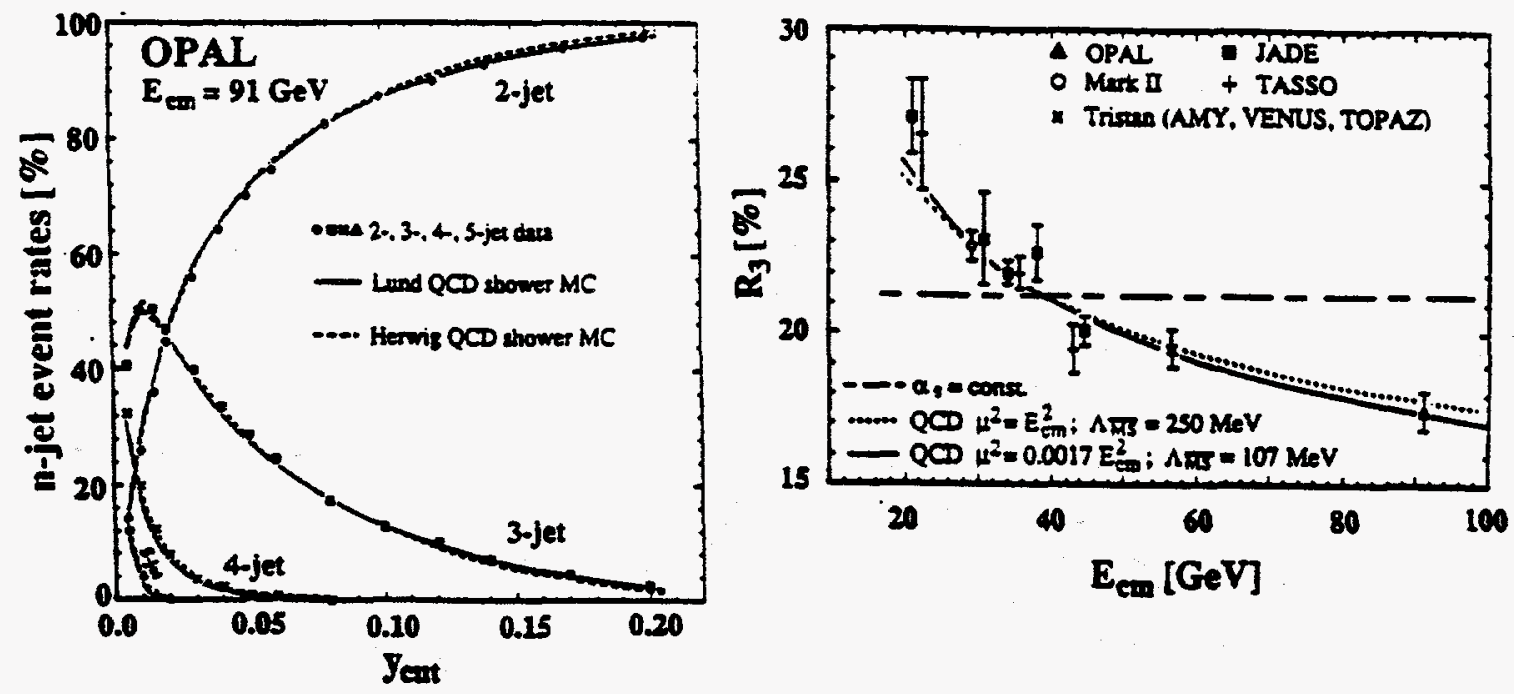

Figure 2.14: left Multijet production rates versus $y_{\text {eut }}$, and right $\alpha_{\text {, evaluated at }}$ various energies and for $y_{\text {cut }}=0.08$

\section{Jet Production and the Running of $\alpha_{*}$}

The production rates of multijet final states in hadronic $Z^{0}$ decays are of particular interest. The relative production rates of multijet events are determined by the value of the strong coupling constant $\alpha_{2}$ which is expected to decrease with increasing energy according to the concept of asymptotic freedom. Experimental measurements of jet production are well suited to test QCD and to determine $\alpha_{s}$.

We have measured the relative production rates of multijet final states in hadronic decays of the $Z^{0}$ using the JADE jet finding algorithm [17] which has the important feature of close agreement between the number of jets reconstructed at the hadron level and at the parton level over a wide range of c.m. energies. In this method, resolvable jets of particles are defined by demanding that in each event the scaled jet pair masses $y_{i j}$ exceed a threshold value $y_{\text {cut }}$ :

$$
y_{i j}=M_{i j}^{2} / E_{v i s}^{2} \geq y_{\text {cut }}
$$

where $M_{i j}$ is the jet pair mass and $E_{v i s}$ is the total visible energy of the event. Details are discussed in the published article. [18]

The relative production rates of 2-, 3-, 4-, and 5-jet events as a function of $y_{\text {cut }}$ is given in the left half of Figure 2.14 . As $y_{\text {cut }}$ increases, the 3-, 4-, and 5-jet rates decrease since the minimum invariant mass between jets is larger. Also shown are the rates predicted by Jetset and Herwig, in good agreement with the data.

From the figure we observe that the 3 -jet rate $R_{3}$ at $91 \mathrm{GeV}$ is about $17 \%$ for a $y_{\text {cut }}$ value of 0.08 . This same point is shown in the right half of the figure as the 
data point at $E_{c . m}=91 \mathrm{GeV}$, in comparison with measurements of $R_{3}$ by $e^{+} e^{-}$ experiments at lower energies using the same jet finding algorithm and value of $y_{\text {cut }}$. A clear scaling violation is observed: the 3 -jet rate decreases as the c.m. energy increases. This can be interpreted in the context of QCD as being entirely due to the running of the strong coupling constant $\alpha_{s}$. The prediction of the 2nd order QCD calculation of Kramer and Lampe [19] is also shown in the figure for two choices of the renormalization scale $\mu$. In principle, if the calculations are done to all orders, there should be no dependence on $\mu$. The similarity of the two curves demonstrates the insensitivity of $R_{3}$ to the choice of this scale. In contrast, the hypothesis that $\alpha$, does not depend on energy results in a constant rate for 3-jet events. This leads to a value of $\chi^{2} /$ d.o.f. of $45.8 / 8$ for c.m. energies above $25 \mathrm{GeV}$. The hypothesis that $\alpha_{s}$ is constant is thus ruled out with a significance of 5.7 standard deviations.

The measured jet rates at $91 \mathrm{GeV}$ have been used by OPAL to adjust the values of $\Lambda_{\overline{M S}}$ and $\mu^{2}$ in order $\alpha_{1}^{2}$ QCD. We obtain $f=\mu^{2} / E_{c m}^{2}=0.001-0.003$ and $\Lambda_{\overline{M S}}=80$ - $180 \mathrm{MeV}$, or if $f=1$, then $\Lambda_{\overline{M S}}=200-450 \mathrm{MeV}$. A recent preliminary analysis takes into account the effect of different definitions of resolvable jets, the so-called "recombination schemes" to obtain a value of $\alpha_{s}$ that incorporates all theoretical uncertainties due to recombination and renormalization scale. This leads to a value

$$
\alpha_{s}\left(m_{Z}\right)=0.116 \pm 0.016
$$

\section{Angular Correlations in 4-jet Final States}

Four-jet final states of hadronic $Z^{0}$ decays are analyzed in terms of observables that are sensitive to the non-abelian gauge structure of QCD. One of the manifestations of this structure is the gluon-gluon self coupling, which would give rise to the threegluon vertex (TGV) as one of the diagrams responsible for the production of four-jet events. This is shown as diagram (a) in Figure 2.15. Within the abelian vector gluon theory, four-quark final states, shown in diagram (d), are predicted to be produced more often than in the case of QCD. The relative contribution $R_{q \bar{q} q \bar{q}}$ of four-quark final states normalized to the number of four-jet events, as determined by the jet finding algorithm described earlier, is about $4.7 \%$ for QCD but $31.4 \%$ for the abelian theory.

Observables that are sensitive to the different spin structures of events coming from diagram (a) - a gluon of spin 1 decaying to two spin 1 particles - and from diagram (d) - a spin 1 gluon decays to two spin 1/2 particles - have been proposed to reveal the existence of the TGV. The two observables used in the OPAL study, $\chi_{B Z}$ and $\theta_{N R}^{*}$, both assume that four jets have been identified and ordered according to their energies: $E_{1} \geq \ldots \geq E_{4}$.

- The variable $\chi_{B Z}$ comes from a paper by Bengtsson and Zerwas [20] and is the angle spanned by the jet momenta $\vec{p}_{1}$ and $\vec{p}_{2}$ and by $\vec{p}_{3}$ and $\vec{p}_{4}$. The idea is that for $q q g g$ final states, jets 3 and 4 will typically come from the gluons. 

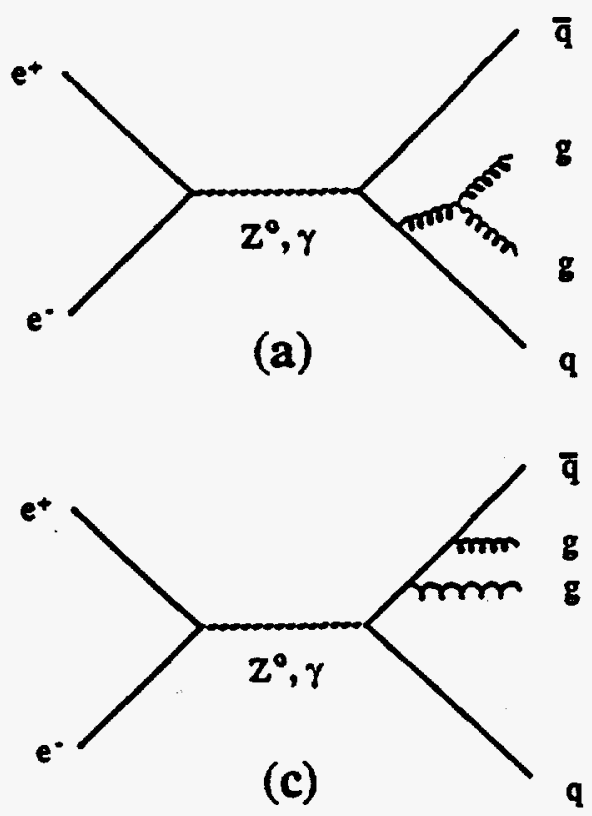
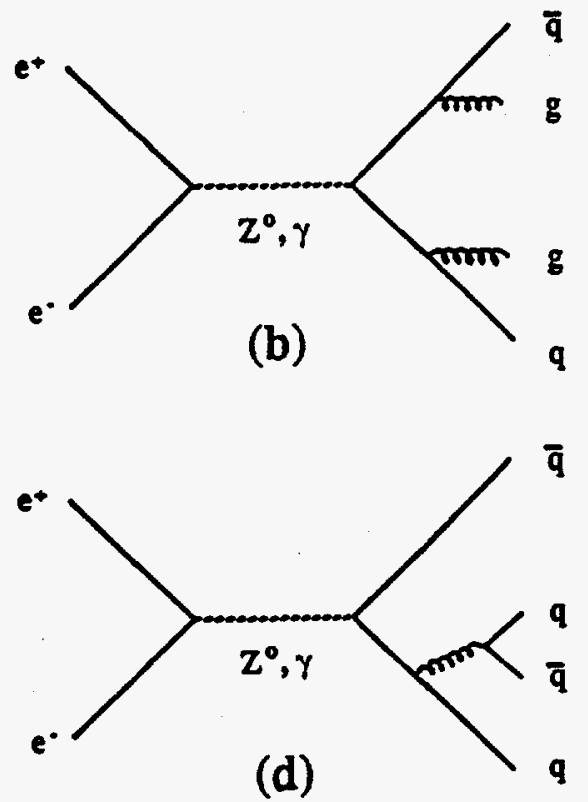

Figure 2.15: Basic Feynman diagrams for the production of four-jet final states.

To make sure the two planes are well-defined, one requires the opening angles between 1 and 2 , and between 3 and 4 each to be less than $160^{\circ}$.

- The angle $\theta_{N R}^{*}$ proposed by Nachtmann and Reiter [21] and modified by Bengtsson [22] is the angle between the vectors $\vec{p}_{1}-\vec{p}_{2}$ and $\vec{p}_{3}-\vec{p}_{4}$.

Comparisons of these two angles as determined from the data and from various abelian and non-abelian models are shown in Figure 2.16. In an analysis where quark and gluon jets are not identified, the observables $\chi_{B Z}$ and $\theta_{N R}^{*}$ are mainly sensitive to the specific spin structure of events where a radiated gluon decays into two fermions, while signatures of the TGV cannot be discriminated from double gluon bremsstrahlung. Thus in this analysis we see a clear difference between the predictions of QCD and the abelian theory, but we cannot isolate and identify the process of gluon self coupling. Nevertheless the good agreement between the measured distributions and the QCD expectations is an important consistency test of QCD since the predicted relative rates of $q \bar{q} q \bar{q}$ final states within the 4-jet sample are significantly different in QCD and in the abelian theory. 

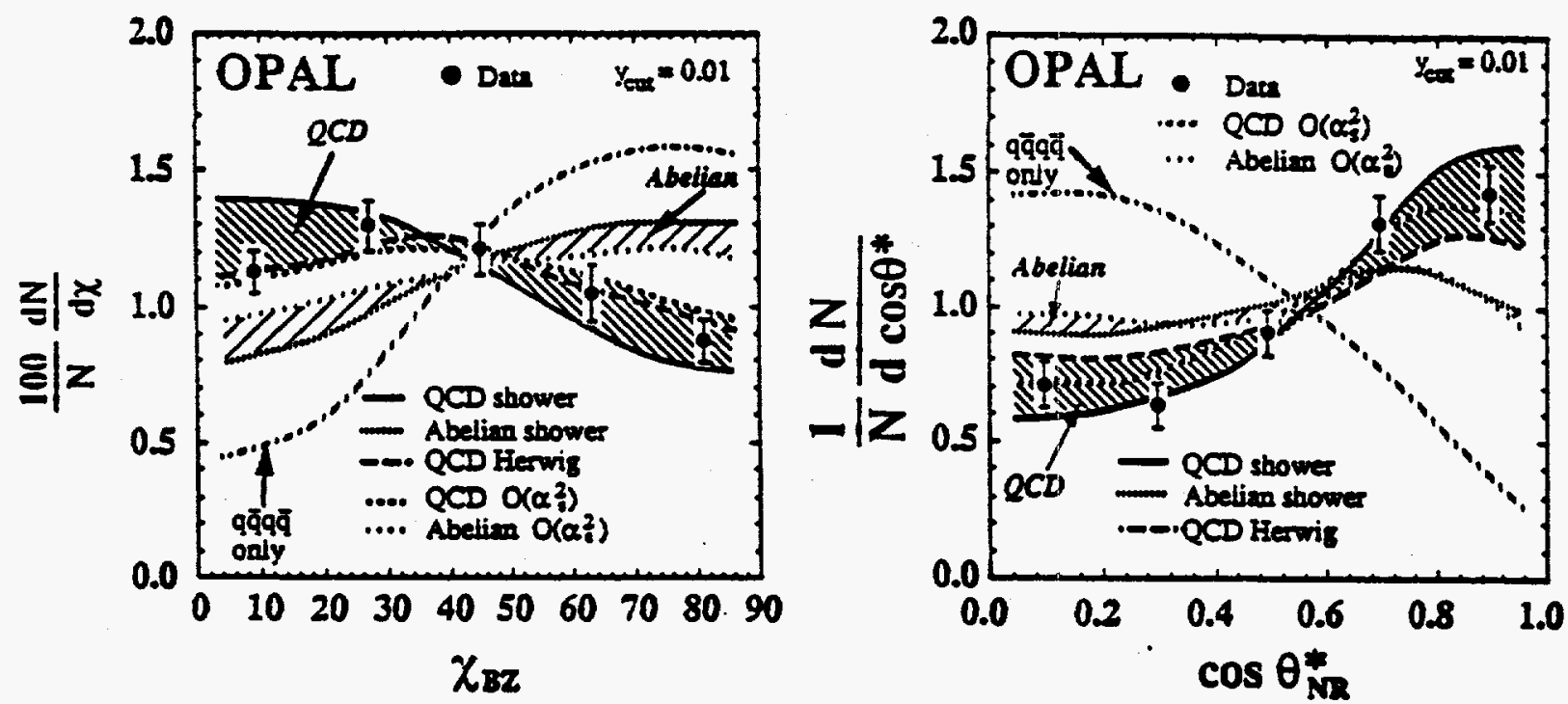

Figure 2.16: Comparison of data and predictions for the Bengtsson-Zerwas angle (left) and the Nachtmann-Reiter angle (right) 


\subsubsection{Final State Photons}

\section{Neutrino counting}

The determination of the number of light-mass neutrino generations is being pursued by the Neutrino Counting Working Group with the participation of Layter, Shen and graduate students Ed Heflin and Bill Larson. OPAL has already determined the number of light-mass neutrino generations by measuring the hadronic $\mathrm{Z}$ width $\Gamma_{z}=2.493 \pm 0.031 \mathrm{GeV}$. Assuming the Standard Model the corresponding number of light-mass neutrino generations is $3.02 \pm 0.15$. This method assumes that the only contribution to any increased width is from a new neutrino generation, whereas a number of candidates, e.g. SUSY particles, can be suggested.

The Neutrino Counting Working Group is using another method first suggested by $\mathrm{Ma}$ and Okada in 1978 [23]. This method involves the "direct" observation of the reaction $e^{+} e^{-} \rightarrow Z \rightarrow \nu \bar{\nu}$ by observing single photons from initial state radiation. Because it is a direct measurement and because it is sensitive to the radiative production of any otherwise undetectable particle pair, the single photon method provides an excellent complementary confirmation of the results of the width method.

The most difficult background is from radiative Bhabhas where both the electron and the positron escape down the beampipe. But a high $P_{T}$ cut on the photon will force either the electron or the positron into the acceptance. Because the most common reaction is the case of either the electron or the positron radiating down to a center of mass energy equal to the $\mathrm{Z}$ mass, the differential cross section versus photon energy has a peak at $E_{C M}-m_{Z}$. This peak is quite prominent and easy to separate from the background as $E_{C M}-m_{Z}$ gets above $4 \mathrm{GeV}$. Unfortunately because LEP has fallen short of its luminosity goal for 1990, a decision was made to run LEP in 1990 mainly at $m_{Z}$ and to scan over a $3 \mathrm{GeV}$ range above and below the $\mathrm{Z}$. Hence the radiative neutrino counting measurement has not yet received adequate running conditions.

A major problem is triggering on these events. A typical $\nu \bar{\nu} \gamma$ event will have no tracks in the central tracking chambers (CD), no energy deposited in the hadron calorimeter (HCAL), no hits in the muon chambers and only one low energy cluster in the electromagnetic calorimeter (ECAL). There are now two signals which will trigger the detector for this type of event. For purposes of the trigger the OPAL detector is divided into 6 theta and 24 phi bins. A deposit of $2.5 \mathrm{GeV}$ or more in one theta-phi bin in the electromagnetic calorimeter or a coincidence of a deposit of $1 \mathrm{GeV}$ or more in one theta-phi bin in the electromagnetic calorimeter and a hit in the same theta-phi bin of the Time of Flight (TOF) triggers OPAL.

As the events are processed, some of them are stripped off to to a separate low multiplicity preselection stream. Single photons candidates are included in this stream. Candidates must meet the following requirements: 
- Multihadron veto: Number of CD tracks + number of ECAL clusters $<17$

- Minimum energy requirement: i) Inside a $300 \mathrm{mrad}$ cone around the largest cluster either; ii) Energy sum $>1 \mathrm{GeV}$ in ECAL barrel; iii) Transverse energy sum $>1 \mathrm{GeV}$ in ECAL endcap

- Cosmic veto: i) No muon segment in Muon chambers or in HCAL barrel; ii) Fewer than 2 strip hits in the outer 8 layers of HCAL number of the HCAL in any 45 degree wedge.

Then the entire low multiplicity preselection was searched for single photon candidates with the following cuts:

- Minimum energy: $1 \mathrm{GeV}$ in ECAL above 40 degrees

- Isolation: no ECAL cluster with energy $>1 \mathrm{GeV}$ outside of a 45 degree cone centered on the the largest cluster.

The events which passed these cuts were scanned to eliminate residual cosmics.

For all of the seven scan energies $3 \mathrm{pb}^{-1}$ of Monte Carlo $\nu \bar{\nu} \gamma$ events with a full detector simulation have been generated. One run at center of mass energy three $\mathrm{GeV}$ above the $\mathrm{Z}$ mass has been carefully searched for single photons. One event, shown in Figure 2.17 , has been found using the selection given above, which is in qualitative agreement with the Monte Carlo prediction of 1.6 events. The group is in the process of analyzing the preselected events at other energies.

In order to interpret the results, it is necessary to understand all other processes which lead to a single photon in the final state. The most important of these background processes is the radiative Bhabha process $e^{+} e^{-} \rightarrow e^{+} e^{-} \gamma$ in which the electron and positron are scatttered at small angles and therefore escape detection, but the photon is seen in the fiducial volume of the detector. Since the process is quantum electrodynamic in nature, it can be predicted with remarkable precision through application of QED to standard Monte Carlo methods. Many Monte Carlo algorithms exist which model the QED radiative Bhabha process, e.g., OLDBAB, BABAMC, HOWLEEG, BHLUMI, TEEGG, etc.

The TEEGG Monte Carlo [24] is considered the most comprehensive Monte Carlo for radiative Bhabha scattering at low $Q^{2}$ and includes radiative corrections and hard brehmsstralung processes. Therefore, to test the QED prediction one must test the Monte Carlo with at least one other particle seen in the final state. To this end, the radiative Bhabha process $e^{+} e^{-} \rightarrow \gamma e^{ \pm}\left(e^{\mp}\right)$ was considered in which a single photon is observed in the electromagnetic calorimeter and an electron or a positron is observed in the central detector while the other positron or electron scatters at a small angle and escapes detection. Such events are characterized by extremely coplanar events with respect to the photon, the observed electron or positron, and the beam direction. In addition, the photon and the observed lepton are expected 


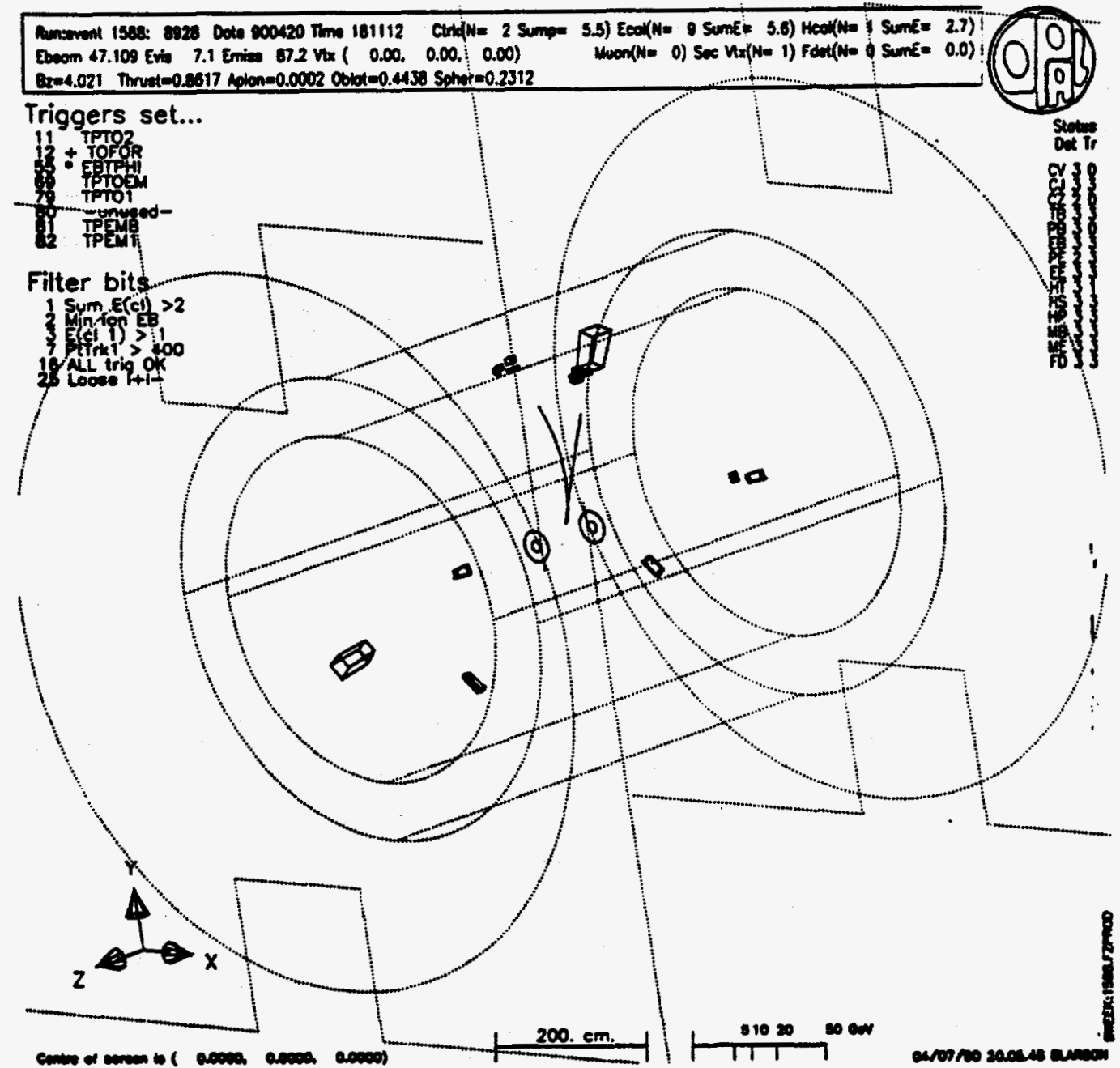

Figure 2.17: Candidate $\nu \bar{\nu} \gamma$ event 
to be very accolinear while depositing nearly half of the center of mass energy in the detector.

An analysis of the single photon and electron/positron final state of all the LOWMP data set from 1989 (periods 1-7) has been completed and compared with the TEEGG Monte Carlo predictions. The events were chosen through cuts on the central detector (CJ) for the observed electron or positron including: i) track quality: number of CJ hits $\geq 20$; ii) vertex position: $\left|d_{0}\right| \leq 0.6 \mathrm{~cm}$ and $\left|z_{0}\right| \leq 30.0$ cm; iii) angular acceptance: $|\cos \theta|<0.9$; iv) uniqueness: no other track fulfilling previous conditions seen in CJ.

Cuts on the electromagnetic calorimeter (ECAL) for the single photon include: i) cluster energy: $E_{\text {raw }} \geq 0.2 E_{\text {beam }}$; ii) angular acceptance; $|\cos \theta|<0.9$; ii) acoplanarity acceptance: $\left|\theta_{\text {acopl }}\right| \leq 0.200$ radians; iv) acolinearity acceptance: $\left|\theta_{\text {acol }}\right| \leq$ 0.200 radians; v) uniqueness: no other ECAL cluster fulfilling previous conditions seen in ECAL

From the complete LOWMP data set for 1989, fifteen events are accepted by the analysis algorithm, EGSEL, implementing the above criteria when good detector and trigger status are required. The integrated luminosity for the good LOWMP data set for 1989 corresponds to $\mathcal{L}=926.7 \pm 20.4 \mathrm{nb}$. Therefore, the observed total cross-section corresponds to

$$
\sigma_{\text {lowmp }}=16.2 \pm 4.2 \mathrm{pb} \text {. }
$$

Applying the same criteria to a sample of 250 TEEGG Monte Carlo events, one finds that 120 events are accepted. For the TEEGG data set, the total cross-section corresponds to

$$
\sigma_{\text {teegs }}=16.4 \pm 2.0 \mathrm{pb} \text {. }
$$

In addition, the superimposed histograms for the two data sets, shown in Figure 2.18 and including angular distributions (lepton and photon), energy distributions, acoplanarity (between lepton and photon), accolinearity (between lepton and photon), etc. agree quite well.

Therefore, the comparison of the 1989 LOWMP data set with the QED predictions given by the TEEGG Monte Carlo yield very good agreement for the radiative Bhabha process in which a single photon and an electron or positron are observed in the detector but the other beam particle escapes detection, scattering at a small angle. Hence, one can be confident that the extrapolation to the radiative Bhabha process in which both the electron and positron are unobserved but the photon is observed is correctly predicted by the TEEGG Monte Carlo.

\section{Final State Photons in Hadronic Decays}

One of the major goals in the study of $Z^{0}$ decays is the determination of the couplings of the fundamental fermions to the $Z^{0}$. Since photons couple to the square of the 

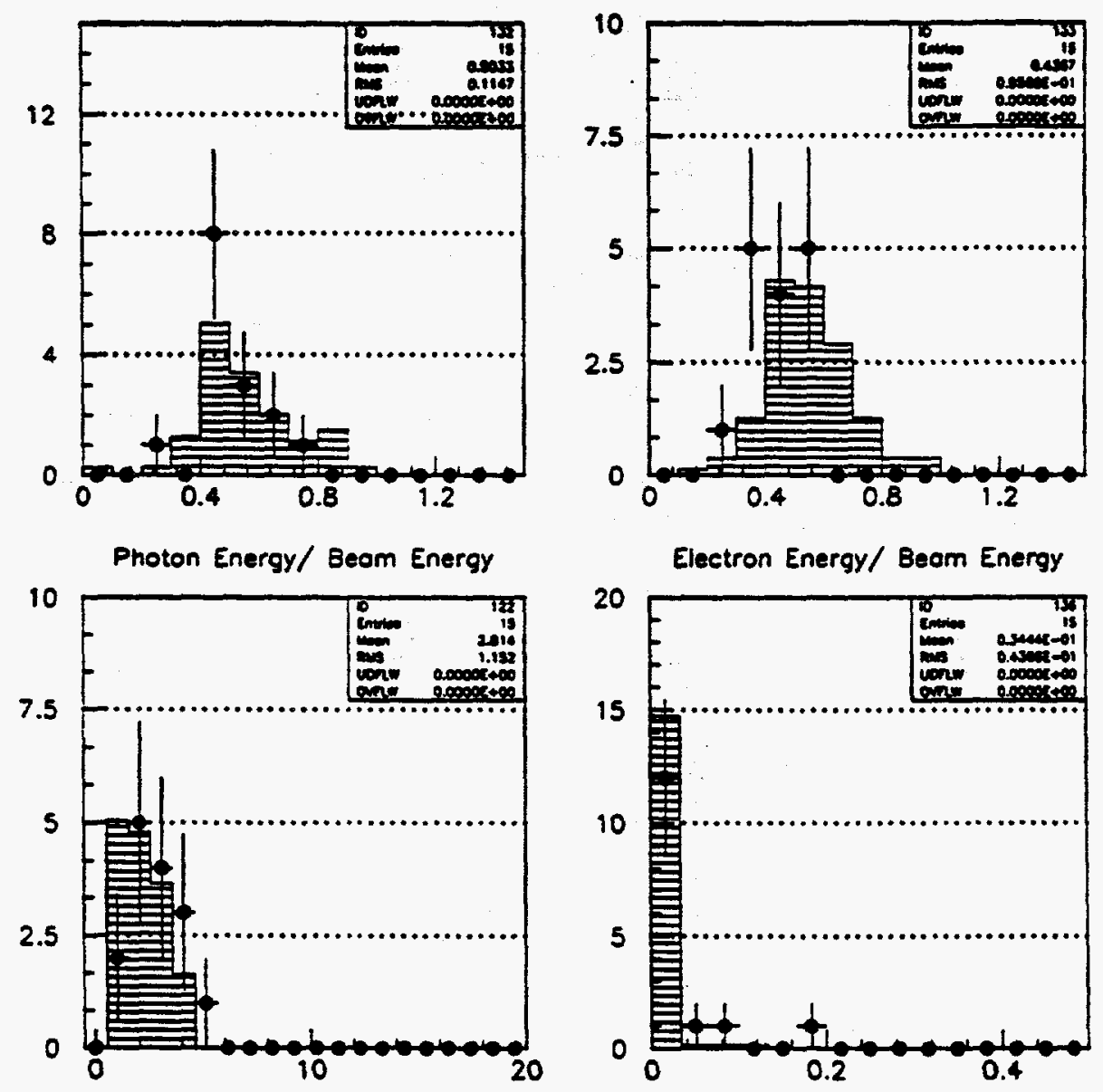

Num of Evnts vs. Num of ECAL Blks (Phot w/90x) Sum Hodronic Energy/ C.M. Energy

Figure 2.18: Histograms of various distributions for TEEGG and LOWMP 


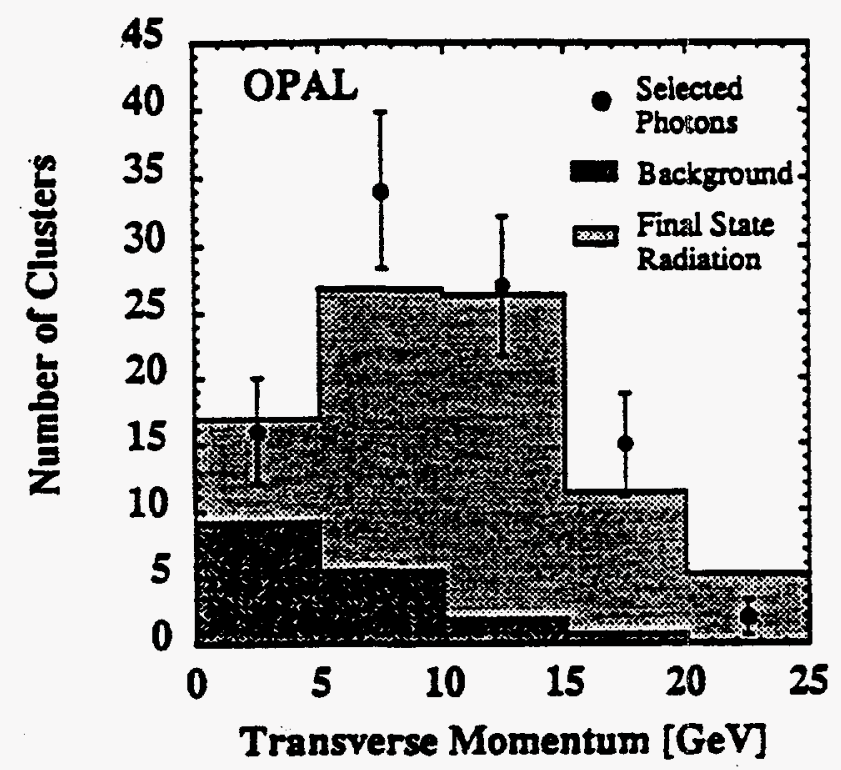

Figure 2.19: Photon transverse momentum

electric charge of the quark, the fraction of charge $2 / 3$ quarks is enriched in a sample containing final state photons. Measuring the rate of final state radiation in $Z^{0}$ decay into hadrons thus provides a simple way to disentangle the weak coupling of up- and down-type quarks.

Assuming that all d-type and u-type quarks are produced equally, the hadronic width is given in the Standard Model in first order QCD as

$$
\Gamma_{h a d}=N_{c} \frac{G_{\mu} M_{Z}^{3}}{24 \pi \sqrt{2}} \cdot\left(1+\frac{\alpha_{a}}{\pi}\right) \cdot\left(3 c_{d}+2 c_{u}\right)
$$

where $N_{c}$ is the number of colors, $G_{\mu}$ the muon decay constant, and the $c_{i}=$ $v_{i}^{2}+a_{i}^{2}$ are the magnitudes of the weak couplings of up- and down-type quarks. The radiative width in first order QED is

$$
\Gamma_{q \varangle \gamma}=N_{c} \frac{G_{\mu} M_{Z}^{3}}{24 \pi \sqrt{2}} \cdot \frac{3}{4 \pi} \cdot \frac{\alpha}{9}\left(3 c_{d}+8 c_{u}\right)
$$

If $\Gamma_{\text {had }}$ and $\Gamma_{q \Phi 7}$ are measured, one can solve for $c_{u}$ and $c_{d}$.

OPAL has measured the radiative width by selecting multihadron events having an isolated photon of a least $10 \mathrm{GeV}$ energy lying at least 20 degrees in angle from any charged or neutral particles. In a sample of over 77000 multihadrons, 78 are found to satisfy the selection criteria. The transverse momentum spectrum of the photon with respect to the thrust axis is shown in Figure 2.19 for these events. Also shown are the predictions of the Jetset Monte Carlo program, with and without final state radiation. The Monte Carlo prediction without final state radiation is 


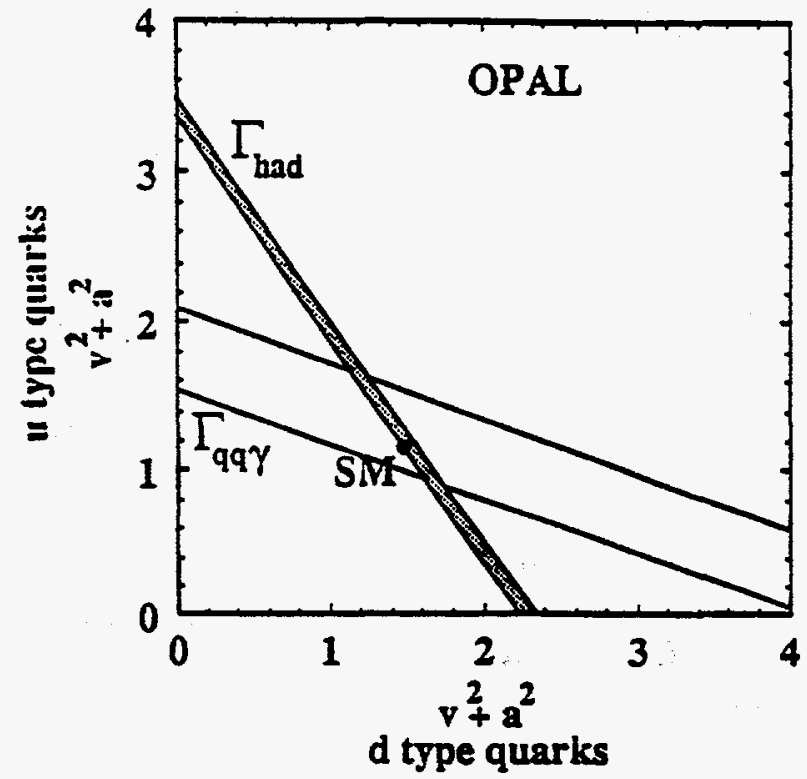

Figure 2.20: Extraction of the coupling constants

consistent with background and is shown as such in the figure. We conclude that we observe direct evidence for final state radiation from quarks.

The extraction of the electroweak coupling constants of u-type and d-type quarks is illustrated in Figure 2.20 where the errors in each measurement are shown. The values of the coupling constants determined in this study are

$$
c_{d}=1.43 \pm 0.25 \text { and } c_{v}=1.28 \pm 0.37 \text {. }
$$

These values can be compared to the Standard Model expectations of 1.48 and 1.15 respectively. Expressing these in terms of partial widths, we have

$$
\Gamma_{d}=369 \pm 67 \mathrm{MeV} \text { and } \Gamma_{u}=330 \pm 89 \mathrm{MeV}
$$

Again the Standard Model expectations are 389 and $301 \mathrm{MeV}$ respectively. The OPAL results are compared with those of the other LEP experiments in Figure 2.21. The results for the other three experiments are obtained using the "traditional" methods of lepton and $D^{*}$ tagging, the errors of which are currently dominated by the semileptonic branching ratios. The error in the method of photon analysis used by OPAL is dominated by the background subtraction, and this can be expected to fall with statistics faster than those of the branching ratios.

The reaction $e^{+} e^{-} \rightarrow \gamma \gamma$

A recent update to the study of the QED reaction $e^{+} e^{-} \rightarrow \gamma \gamma$, already reported by the Collaboration earlier this year [25], now extends the limits on the cutoff 

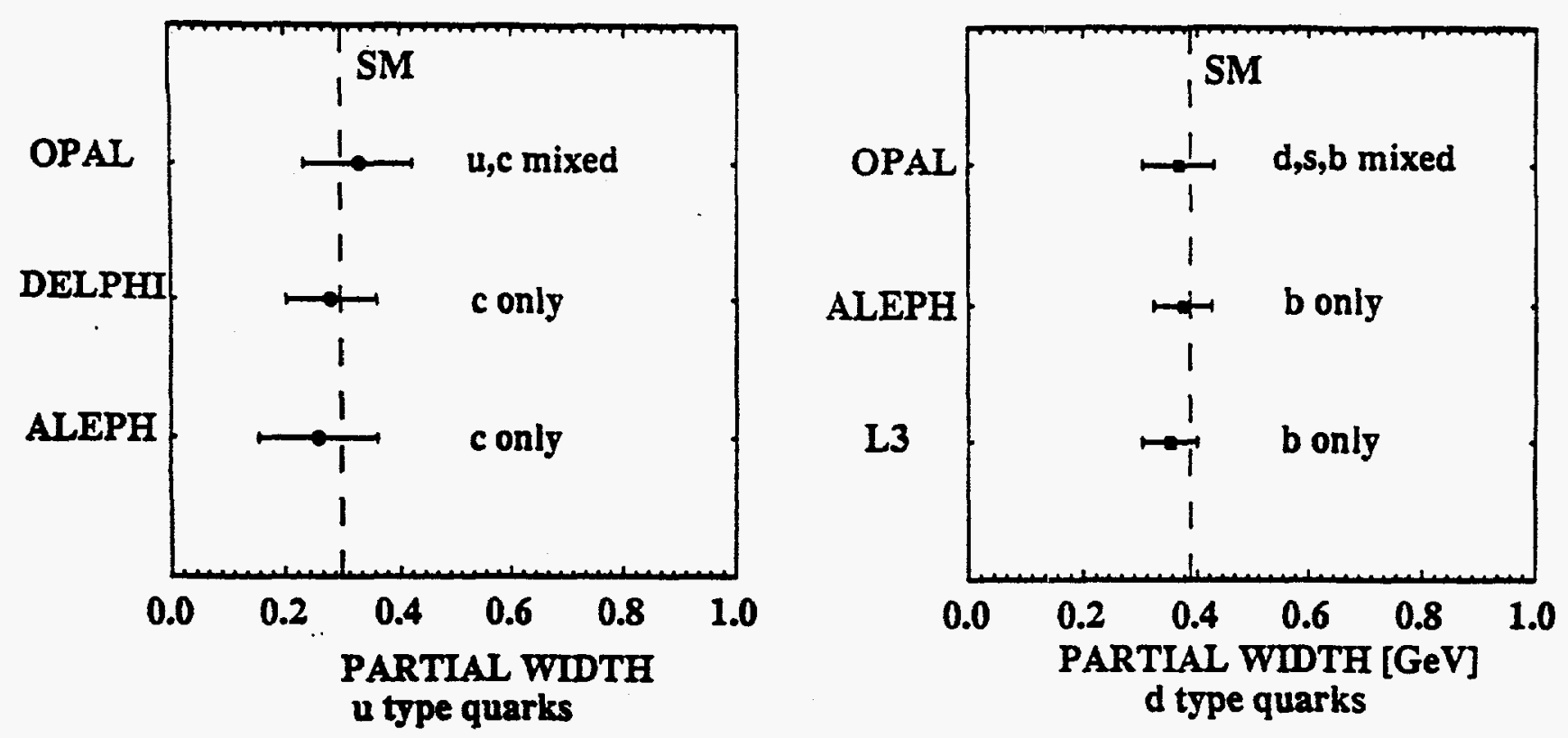

Figure 2.21: Results on quark partial widths compared

parameters and branching ratios. In a sample which corresponds to an integrated luminosity of $1.982 \mathrm{pb}^{-1}$, there are $56 \mathrm{e}^{+} e^{-} \rightarrow \gamma \gamma$ and $2 e^{+} e^{-} \rightarrow \gamma \gamma \gamma$ events. Correction is made for photon conversion in the material of the detector.

The differential cross section for the $e^{+} e^{-} \rightarrow \gamma \gamma$ reaction is shown in Figure 2.22. A discrepancy of two standard deviations is seen in the smallest angle bin, but no detector failure has been uncovered and this is attributed to a fluctuation. From this cross section we obtain lower limits on the cutoff parameters of $\Lambda_{+}>103 \mathrm{GeV}$ and $\Lambda_{-}>92 \mathrm{GeV}$ at $95 \%$ confidence level. Upper limits on widths and branching ratios for some rare decay modes are given in the table.

\begin{tabular}{||l|c|c||}
\hline Reaction & Width (MeV) & Branching Ratio \\
\hline$Z^{0} \rightarrow \gamma \gamma$ & 0.44 & $<1.7 \times 10^{-4}$ \\
$Z^{0} \rightarrow \pi^{0} \gamma$ & 0.45 & $<1.8 \times 10^{-4}$ \\
$Z^{0} \rightarrow \eta \gamma$ & 0.69 & $<2.6 \times 10^{-4}$ \\
$Z^{0} \rightarrow \gamma \gamma \gamma$ & 0.13 & $<5.2 \times 10^{-5}$ \\
\hline
\end{tabular}




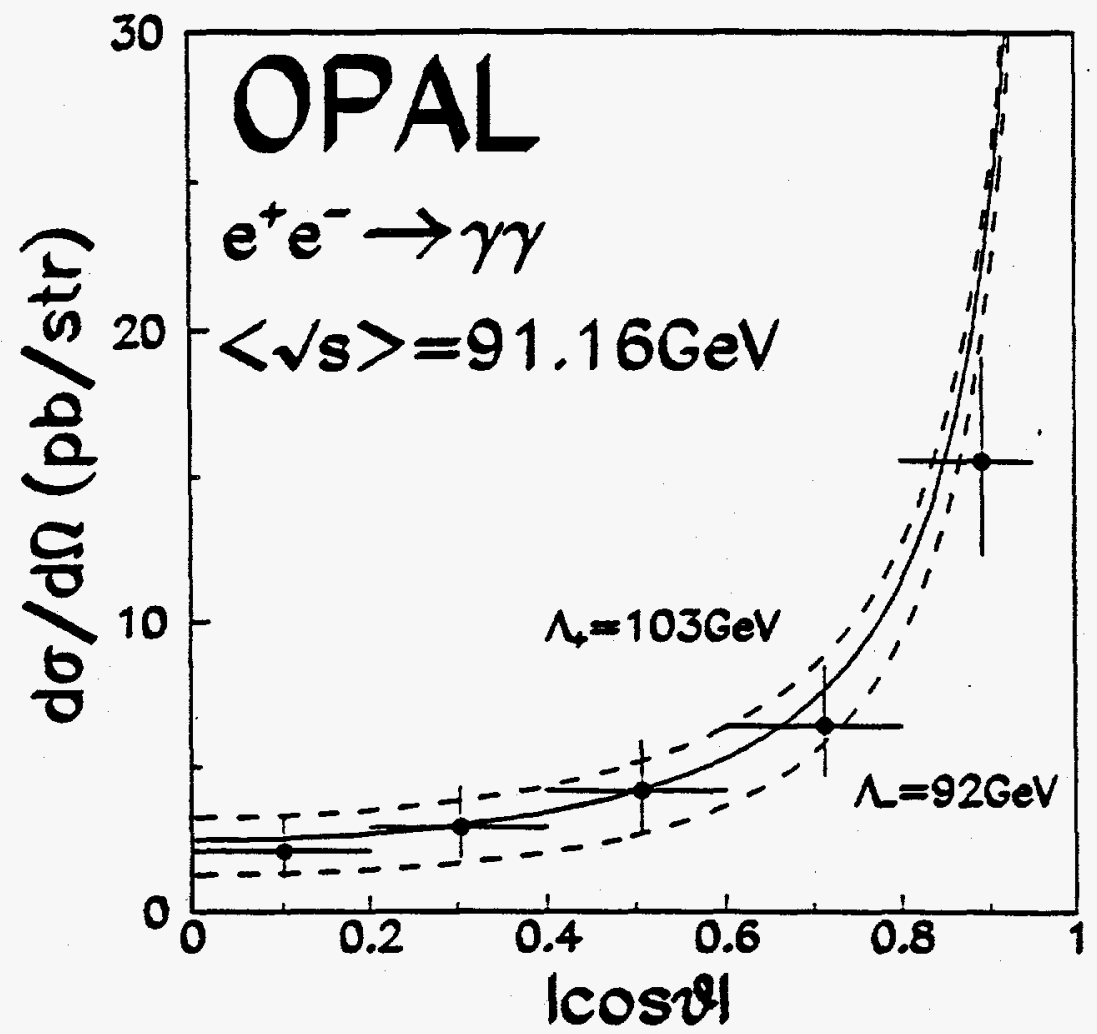

Figure 2.22: Differential cross section for $e^{+} e^{-} \rightarrow \gamma \gamma$ 


\subsubsection{Studies of $\tau^{+} \tau^{-}$Pair Production and Decay}

The heaviest known lepton, the $\tau$, can be used as a laboratory to study electroweak phenomena and answer some fundamental questions. The main points of interest are:

- Lepton universality

- $\tau$ lepton number conservation

- $\tau$ neutrino mass

- The determination of the $\tau$ couplings to the $\mathrm{W}$ and the $Z^{0}$

- Indirect searches for new interactions and massive particles (right handed W's and extra $Z^{0}$ 's )

- Searches for exotic particles decaying into $\tau$ 's.

Experiments at LEP can give detailed answers to the above questions. As can be seen from Figure 2.23 the expected number of $\tau^{+} \tau^{-}$(and $b \bar{b}$ ) events at LEP is clearly competitive with previous $e^{+} e^{-}$experiments at lower energies.

During the last year, the OPAL collaboration has performed several measurements which answer some of these questions. The number of neutrinos has been measured to be $3 \pm 0.2$, [26], giving strong evidence for the $\tau$ neutrino and excluding the existence of more lepton families with small neutrino masses. As discussed earlier, our data give strong confirmation to the concept of lepton universality. We have also searched for $Z^{0}$ decays into exotic particles, like supersymmetric $\tau$ 's, charged Higgses, and heavy neutral leptons which could decay subsequently into $\tau$ 's. [27]. A method - developed for our search for supersymmetry in events with low charged and neutral particle multiplicity - has also been used to search for charged Higgses and to analyze the production of $\tau$ pair events [28]. Recently we have used this method to perform a combined lepton pair analysis [29]. These measurements will continue during the next years and much higher precisions can be expected.

During the 1990 data taking period we expect to increase our statistics by about a factor of ten. The Tau Working Group, which includes M. Dittmar as co-chairman, K. Riles, B. Shen and two graduate students C. Ho and B. O'Neill, has for its main physics goals for this year's data taking:

- Evidence for non-zero forward backward charge asymmetry of $\tau$ 's at the $Z^{0}$ pole.

- The observation of $\tau$ polarization, a proof of parity violation in $Z^{0}$ decays. The observation of $\tau$ polarization, in addition to a precision measurement of $\sin ^{2} \theta_{W}$, makes possible the determination of the absolute sign of the axial and vector couplings of the leptons. 


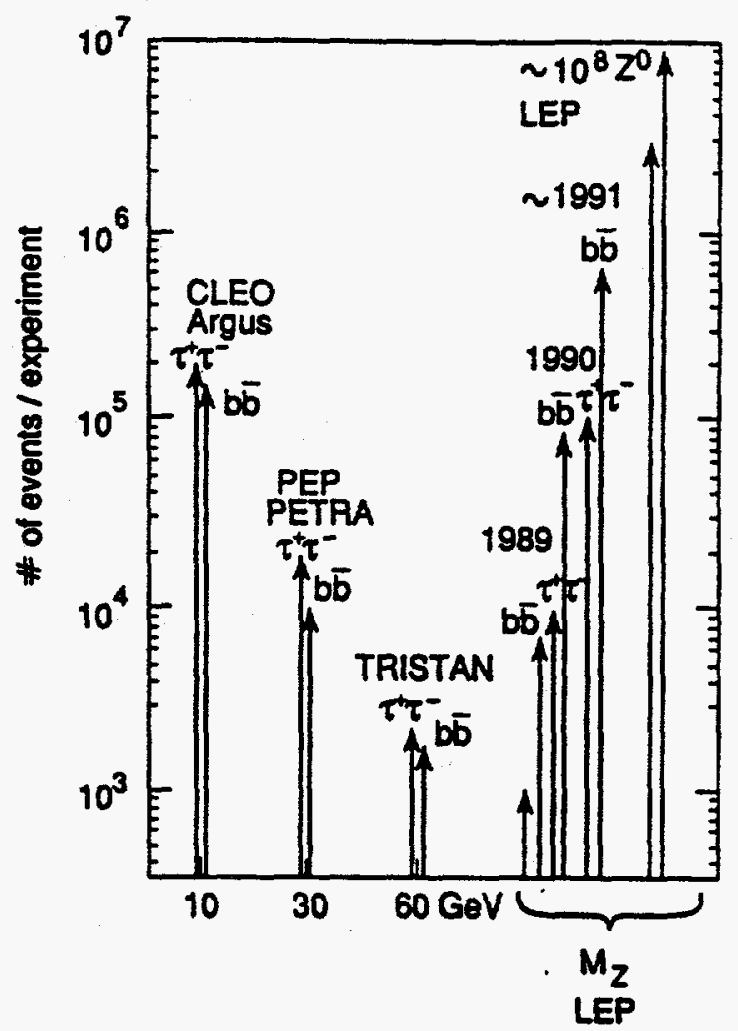

Figure 2.23: Comparison of the expected number of $\tau^{+} \tau^{-}$(and and $b \bar{b}$ ) events at LEP and previous $e^{+} e^{-}$experiments

- The search for violation of the $\tau$ lepton number in $Z^{0}$ decays.

- A precise measurement of $\tau$ branching ratios, which would demonstrate the feasibility of precision $\tau$ studies at the $Z^{0}$ pole.

\section{Selection of Lepton Pair Events}

We have performed an analysis which uses the information from the central detector and the lead glass calorimeter to select the different types of lepton pairs with high efficiency and very small background. As can be seen from Figure 2.24, the different types of lepton pairs $\left(e^{+} e^{-}, \mu^{+} \mu^{-}\right.$, and $\left.\tau^{+} \tau^{-}\right)$are well separated from each other. This combined analysis of the different types of lepton pairs simplifies some measurements considerably, since efficiencies and accuracies can be determined directly from the data. Starting from this lepton pair selection, different detailed studies are now in progress.

\section{Study of the different $\tau$ decay modes}

Considerable effort has been spent in recent years at previous $e^{+} e^{-}$experiments to measure different $\tau$ decay modes. We have analyzed a sample of about $1000 \tau$ decays (collected during 1989) and obtained encouraging results on $\tau$ decays into 1 and 3 


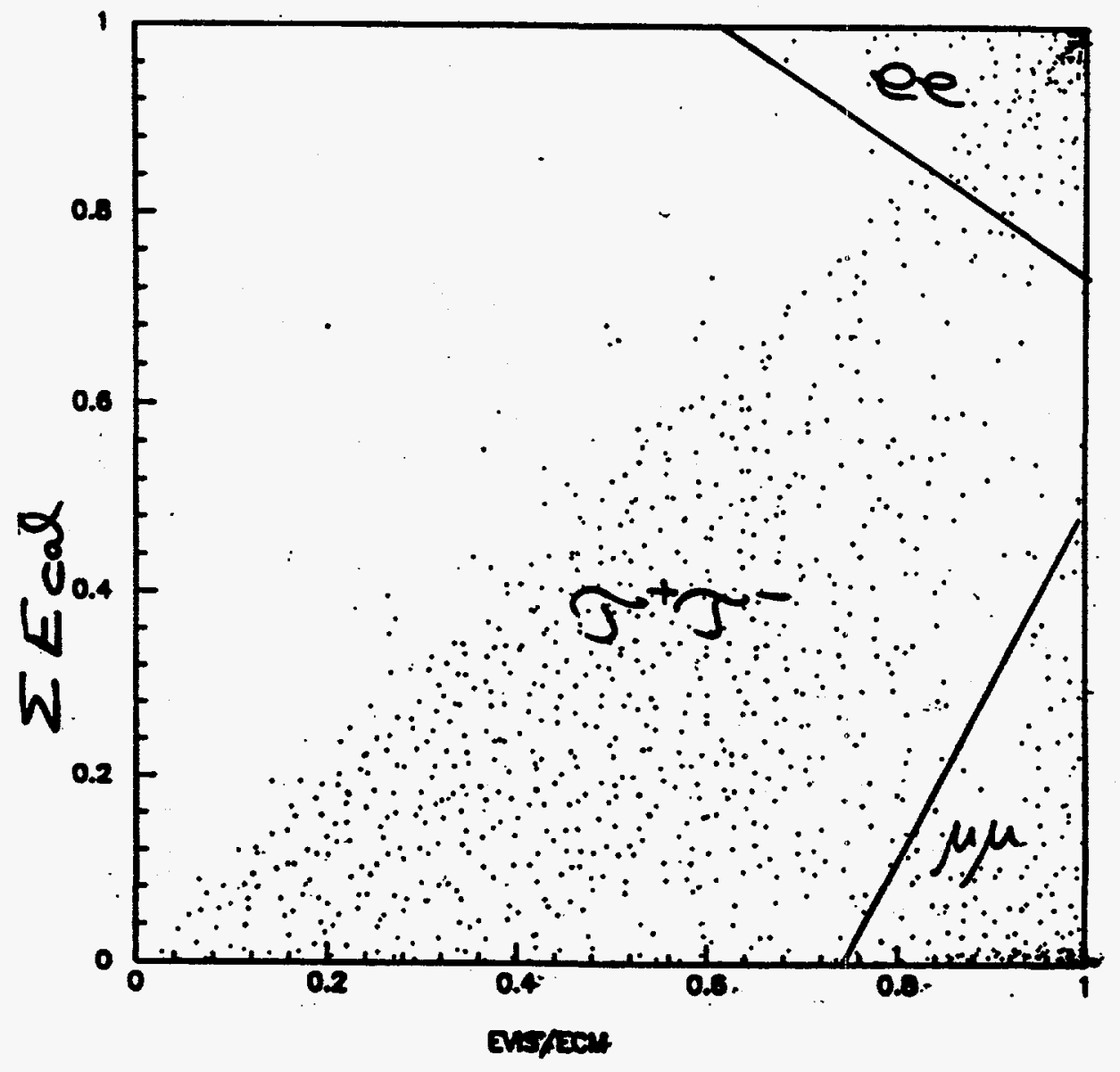

Figure 2.24: Visible energy versus calorimeter energy for OPAL 1989 low multiplicity events 


\begin{tabular}{|c|c|c|c|}
\hline tagged side & opposite side & BR (\%) & PDG 1988 (\%) \\
\hline 1 track & 1 & $84.4 \pm 1.5 \pm 1.2$ & $86.7 \pm 0.4$ \\
\hline 1 track & 3 & $15.1 \pm 1.5 \pm 1.2$ & $13.19 \pm 0.26$ \\
\hline 3 track & 1 & $83.5 \pm 3.1 \pm 2.2$ & $86.7 \pm 0.4$ \\
\hline
\end{tabular}

Table 2.3: Topological branching ratios from OPAL data compared with world averages

charged particles and also for the $\tau$ decay into $\mu$ 's. Topological branching ratios were determined by finding an identified $\tau$ using the criteria just mentioned. This constitutes the "tagging side" of the event, and it can be a one-prong or a threeprong track. One then observes the charged multiplicity on the opposite side of the event. All events with a tagged $\tau$ on both sides were counted twice. All even pronged events were scanned and were found to be perfect candidates for very asymmetric gamma conversions. Therefore, we have grouped the even pronged decays into the corresponding set of the next lower odd pronged decays. We estimate a systematic error of about $30 \%$ for this procedure. Table 2.3 contains the observed branching ratios of the $\tau$ into 1 and 3 charged tracks using the different tagging conditions. Our results, even uncorrected for uncertainties due to unobserved photon conversions (enhancing the 3 charged particle mode), are in perfect agreement with the current world averages on topological branching ratios. [30].

We have also unambiguously identified $\mu$ 's from $\tau$ decays using the information from the hadron calorimeter and the $\mu$ chambers. To determine the $\mu$ efficiency, we use $Z^{0}$ decays into $\mu^{+} \mu^{-}$pairs which allow us to measure efficiencies with very small systematic errors. Our results for the branching ratio using the 1989 data are: $\operatorname{BR}(\tau \rightarrow \mu X)=18.0 \pm 2.0 \%$ This is in excellent agreement with the current world average of $17.8 \pm 0.4 \%$. It should also be compared with the best measurements from single experiments with errors of about $0.8 \%$. Currently we are preparing a publication on these measurements using the increased statistics during the 1990 data taking period. This publication will also include the $\tau$ decay into electrons, for which we have developed some criteria which have high efficiency and low background. Figure 2.25 shows our preliminary results for the $\mu$ momentum spectrum, compared with Monte Carlo expectations.

\section{The $\tau$ polarization measurement}

The $\tau$ polarization can be measured using the 2-body $\tau$ decays into spin $0+\operatorname{spin} 1 / 2$ particles $(\pi \nu$ and $\mathrm{K} \nu$ ). Within the context of the standard model the polarization of the $\tau^{-}$for unpolarised beams at the $Z^{0}$ peak is:

$$
P(\theta)=-\frac{2 a_{\tau} v_{\tau}}{a_{\tau}^{2}+v_{\tau}^{2}}-\frac{2 a_{e} v_{e}}{a_{e}^{2}+v_{e}^{2}} \cdot \frac{\cos \theta}{1+\cos ^{2} \theta}
$$




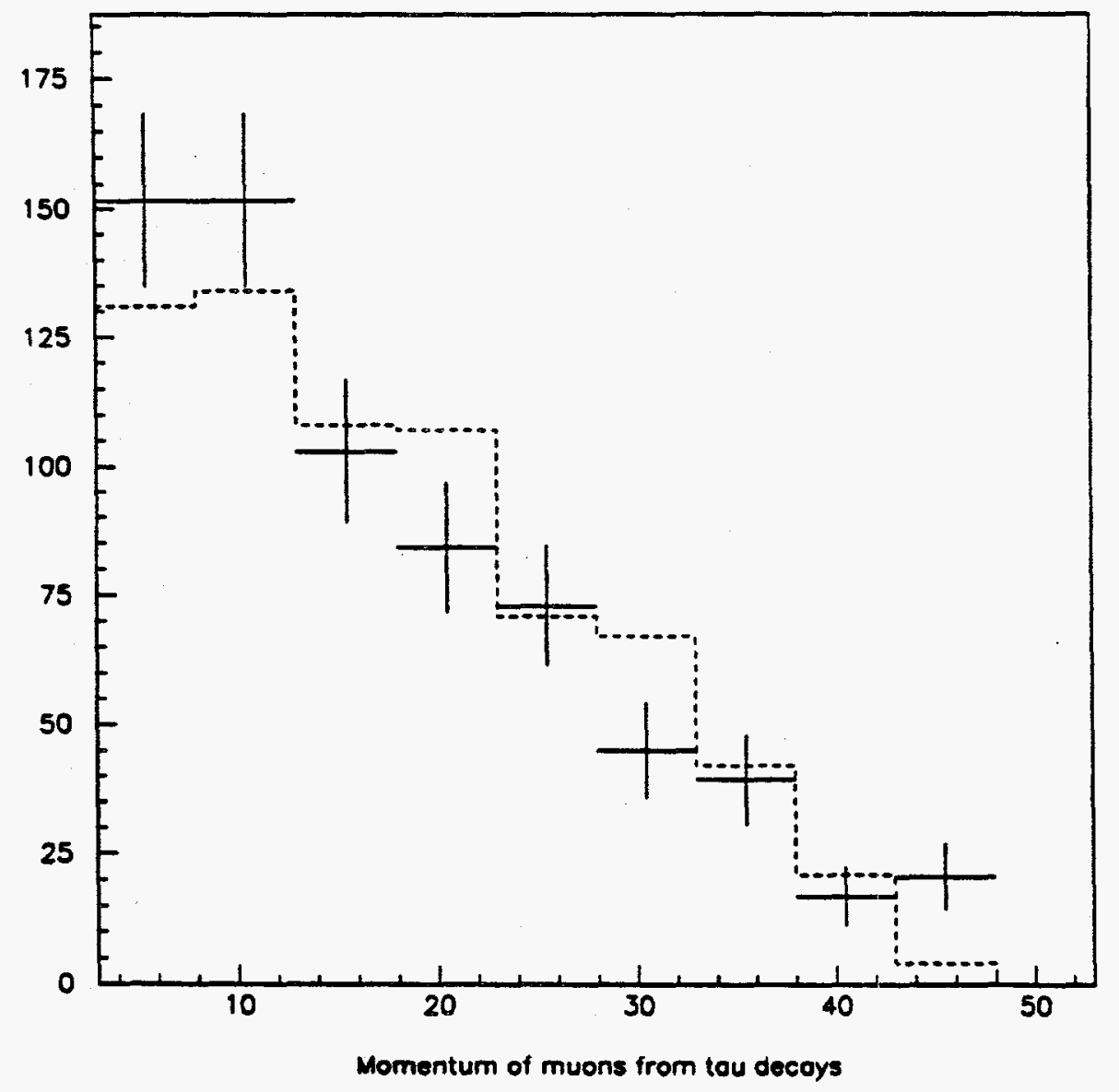

Figure 2.25: The $\mu$-momentum spectrum from $\tau$ decays. The dashed curve is the result from the KORALZ program. 
Thus, within the Standard Model one expects to see three different regions for the $\tau$ polarization: the forward region with about $30 \%$ polarization, the backward region with a polarization close to 0 , and the average polarization of about $15 \%$ for any range symmetric in $\cos \theta_{\tau}$ around 0 . The $\pi$ momentum spectrum can be used to measure the $\tau$ polarization according to:

$$
\frac{d n}{d x_{\pi}}=1+P(\tau)(2 x-1)
$$

Only about $13 \%$ of the 1 prong $\tau$ decays are $\pi(K) \nu$ decays. (In the following we consider the $\pi \nu$ and $\mathrm{K} \nu$ together since we did not try to distinguish the two experimentally.) In addition, the momentum spectrum of the background from $\rho, e$ and $\mu$ decays is peaked at low $x$. These decay modes, if not perfectly rejected, might lead to a non-negligible asymmetry. A further complication appears to be the final state radiation. It reduces the $\tau$ energy and therefore shifts the pion (kaon) energy spectrum to lower $x$ values, leading to an additional asymmetry.

Our analysis aims to get a very clean pion sample with an efficiency almost independent of the momentum. We developed the following criteria to obtain clean events of the type a sample of $\tau$ decays into $\pi(\mathrm{K}) \nu$.

1. A jet should consist of exactly 1 good track with $-0.7<\cos \theta<0.7$, a fractional momentum $x>0.06$ and more than 100 hits to provide an accurate momentum measurement (this excludes sector 1 and 2 were we have observed a systematic shift in the momentum measurement). The lower $\mathrm{x}$ cut was needed to reject $\mu$ 's from $\tau$ decays with good efficiency.

2. There should be exactly 1 electromagnetic cluster close to the track (with a minimum energy of $150 \mathrm{MeV}$ ). This rejects mainly $\tau$ decays with many $\pi^{0}$ 's but also $\pi \nu$ decays with early showers in the coil or with very broad showers in the Lead Glass.

3. To get a further separation between $\pi$ 's and the background we required that there be no more than 1 presampler cluster close to the track with an energy between 0.6 and 6 MIPs. This strongly rejected $\tau$ decays to electrons and $\rho$ 's.

4. The ratio of calorimeter energy relative to the momentum measurement has to be smaller than 0.8 to remove electrons and $\rho$ background.

5. Muons identified by a good match between the track and a muon segment (in $\phi$ only) in either the Hadron Calorimeter or the Muon Barrel detector, about $80 \%$ of all $\mu$ 's, are rejected.

6. To reduce the muon background further, we have required that the ratio between the number of strip hits divided by the number layers with hits be greater than 2. This criterion is applied only if the number of blocks 


\begin{tabular}{|l|r|r|r|r|r|}
\hline cut number & data & $\mathrm{MC} \pi(\mathrm{k})$ & $\mathrm{MC} \mathrm{e}(\mu)$ & $\rho$ & other \\
\hline 1. (1 track) & 912 & $617(50)$ & $1020(1043)$ & 1104 & 682 \\
\hline 2. (1cluster) & 636 & $425(40)$ & $971(997)$ & 365 & 167 \\
\hline 3. (0, 1 PS cl) & 286 & $287(29)$ & $80(970)$ & 20 & 13 \\
\hline 4. (E/p ) & 259 & $279(29)$ & $1(970)$ & 8 & 9 \\
\hline 5. (no muon) & 82 & $262(26)$ & $1(172)$ & 7 & 9 \\
\hline 6. (strips ) & 44 & $228(22)$ & $1(3)$ & 6 & 9 \\
\hline
\end{tabular}

Table 2.4: The effect of the different criteria in the data and the Monte Carlo based on 699 tau events in the data and 3000 in the MC

in the lead glass cluster is smaller than 5. Only $\pi$ 's with no (or late) interaction in the lead glass are affected by this criteria (about $30 \%$ of of all pions).

The resulting number of $\tau$ decays into pion candidates is summarized in Table 2.4 for the 1989 data (699 $\tau^{+} \tau^{-}$events) and for $\tau^{+} \tau^{-}$Monte Carlo events, which are separated into the different decay channels. According to the Monte Carlo we obtain an efficiency of $37.5 \% \pm 2.0 \%$ for $x_{p}$ above 0.1 (about $4.5 \mathrm{GeV}$ ). No momentum dependence is observed. We have used the identified $\pi(\mathrm{K})$ decays to determine the branching ratio of $\tau \rightarrow \pi(\mathrm{K}) \nu$.

After correcting for the efficiency and the momentum range below $4.5 \mathrm{GeV}$ we measure a branching ratio of $\tau \rightarrow \pi(\mathrm{K}) \nu$ of $10.2 \pm 1.8 \%$ (stat). This number should be compared to the current world average of $11.5 \pm 0.6 \%$. We attribute a systematic error of about $10 \%$ for imperfections of the Monte Carlo simulation.

We have continued the analysis for the $1990 \mathrm{data}$, and about 2000 clean $\tau^{+} \tau^{-}$ events have been recorded so far. Using our selection of the $\pi(K)$ decays we find a total of about 150. The observed momentum distribution is in good agreement with the expectation, and first evidence for $\tau$ polarization is seen. The work now concentrates on a publication which should be ready by the end of this running period.

\section{Search for Lepton Flavor Violation in $Z^{0}$ Decays}

We have performed a direct search for $Z^{0}$ decays into $e^{ \pm} \tau^{\mp}$ and $\mu^{ \pm} \tau^{\mp}$ using a sample of about $10^{5} Z^{0}$ decays which have been recorded with the OPAL experiment during 1989 and up to June 1990. Such events can be identified by back to back low multiplicity jets with one electron or muon with an energy close to the beam energy. At least one of the $\tau$ decay products is a neutrino, which carries energy and momentum. The $\tau$ side can therefore be identified by its low visible energy. No 


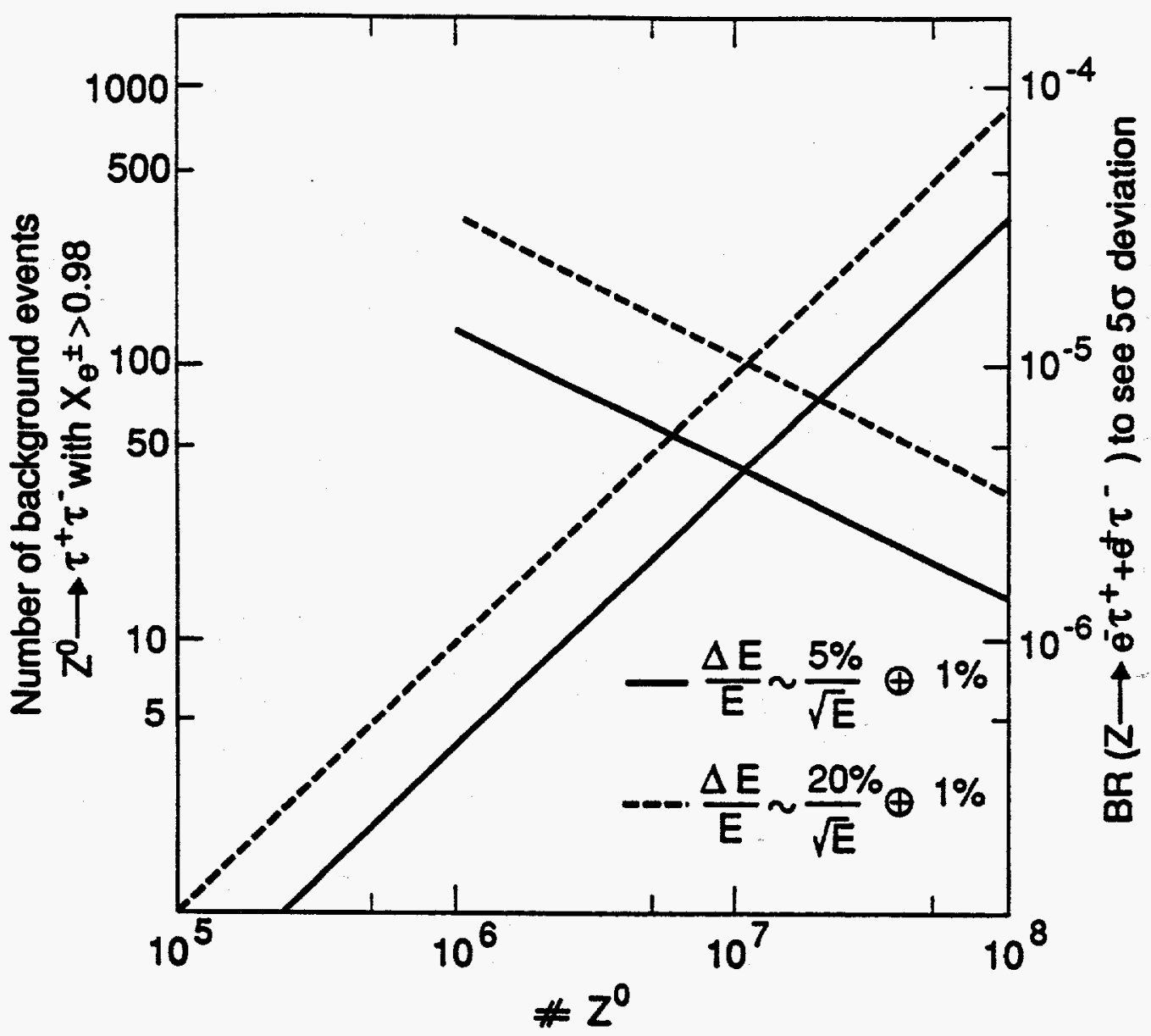

Figure 2.26: The sensitivity to lepton flavor violation in $Z^{0}$ decays as a function of the total number of $Z^{0}$ decays

evidence for such events has been found so far and limits of about $2 \cdot 10^{-4}$ for the lepton flavor violating $Z^{0}$ branching ratios are currently obtained.

\section{Future $\tau$ Analysis Projects}

Our goals on $\tau$ physics are precision measurements of lepton universality, the forward backward charge asymmetry and the $\tau$ polarization. Accuracies in $\sin ^{2} \theta_{w}$ of 0.004 can be expected. We will also continue to perform detailed studies of the different $\tau$ decay modes. The searches for lepton flavor violation and neutrino singlet production will continue in the future. We have studied the sensitivity to such processes for the proposed high luminosity LEP upgrade [31], and the results are indicated in Figure 2.26 


\subsubsection{Searches}

The copious production of $Z^{0}$ at LEP provides an excellent opportunity to search for new particles, some of which are regarded as essential components of the Standard Model, and others are proposed as extensions to the Model. However, in the words of one of our OPAL colleagues, "You name it, we haven't seen it." The Collaboration has performed direct searches and set mass limits for the $t$ and $b^{\prime}$ quarks, sequential heavy leptons, neutral heavy leptons, supersymmetric particles, excited leptons, and Higgs bosons.

\section{$t$ and $b^{\prime}$ Quarks}

OPAL has carried out a search, using 14986 hadronic $Z^{0}$ decay events from the 1989 data run, for the production of heavy quarks $t$ and $b^{\prime}$. Several decay modes are considered: i) charged current decays $t \rightarrow b W^{*}$ and $b^{\prime} \rightarrow c W^{*}$, ii) flavor-changing neutral current decays $b^{\prime} \rightarrow b g$ and $b^{\prime} \rightarrow b \gamma$, and iii) decays via charged Higgs $t \rightarrow$ $b H^{+}$and $b^{\prime} \rightarrow c H^{-}$. The distributions of event shape variables such as acoplanarity, sphericity, and thrust, are sensitive to the presence of new heavy quarks. We have compared the experimental distributions with those from Monte Carlo simulations using Jetset. In all cases we have found good agreement between data and those distributions expected for the production of quarks of five flavors. Details of cuts and selections can be found in the published paper [32]. Mass limits, at $95 \%$ confidence level, are as shown in the accompanying table.

\begin{tabular}{|l|c|}
\hline Decay channel & Mass limit \\
\hline$t \rightarrow b W^{*}$ & 45.1 \\
$b^{\prime} \rightarrow c W^{*}$ & 45.4 \\
$b^{\prime} \rightarrow b \gamma(\mathrm{BR}=0.25)$ & 45.4 \\
$t \rightarrow b H^{+}$ & 45.3 \\
$b^{\prime} \rightarrow c H^{-}$ & 45.5 \\
\hline
\end{tabular}

\section{Heavy Leptons}

The data sample obtained during the fall of 1989 quickly provided OPAL with sufficient statistics to allow immediate searches for charged and neutral heavy leptons. Since the Mark II experiment at SLC had already excluded new quarks and some models of neutral leptons for masses below the beam energy, K. Riles and H. Oh of the Riverside group, together with members of the CERN and Chicago groups concentrated initially on a search for a heavy charged lepton produced via the standard coupling $Z^{0} \rightarrow L^{+} L^{-}$and decaying into a fourth-generation neutrino and conventional particles [33]. All heavy lepton events will contain at least two energetic neutrinos, resulting in large missing energy and transverse momentum. Furthermore, the branching ratios for the three leptonic decays $L \rightarrow \nu_{L}+l+\bar{\nu}_{l}$ are 


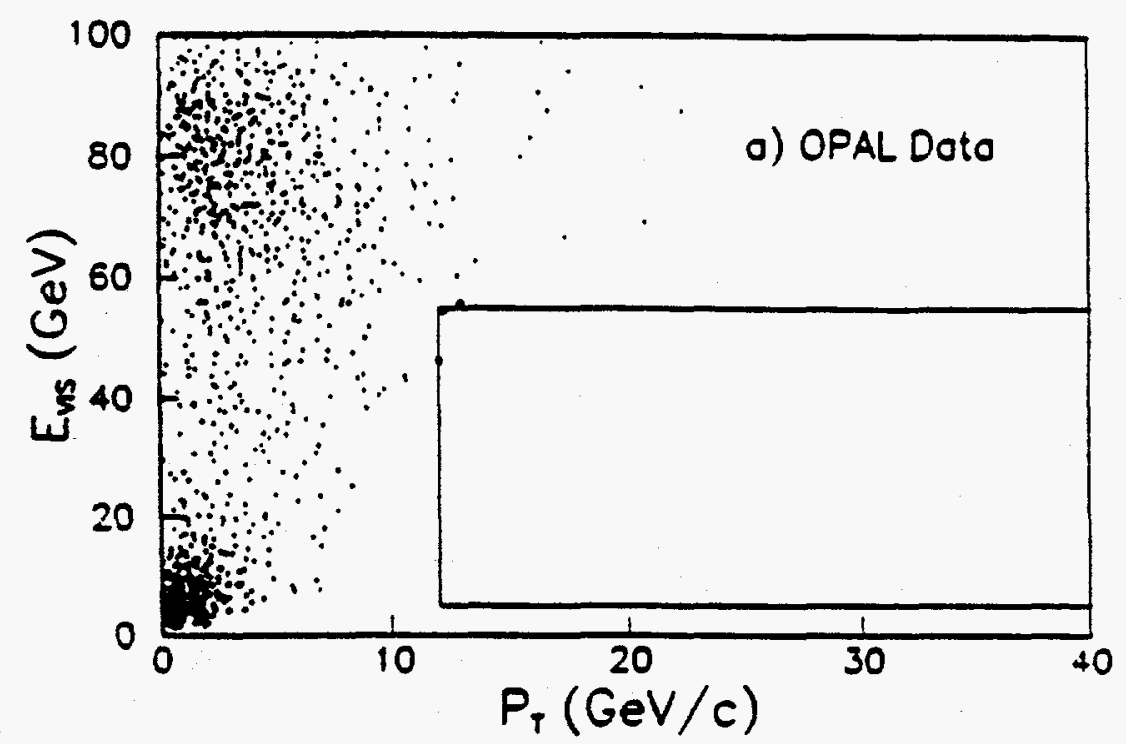

Figure 2.27: Distribution of visible energy versus missing transverse momentum.

$1 / 9$ each. Including the $\tau$ leptonic decays gives a total branching ratio $L^{ \pm} \rightarrow e$ or $\mu$ of $26 \%$. Consequently, $45 \%$ of $L^{+} L^{-}$events will have at least one energetic $e$ or $\mu$.

Two distinct signatures were considered. The first approach selected events with missing energy and missing transverse momentum, since one expects the undetected fourth-generation neutrinos to carry away substantial energy and momentum. Remaining backgrounds from tau-pair production and from mismeasurement of hadronic events were removed by requiring the thrust of each event to be less than 0.95 and by requiring that within a cone of 30 degrees half-angle centered about the missing momentum direction there be less than $1 \mathrm{GeV}$ each of charged and neutral detected energy. After these requirements, two events survived from a data sample of $750 \mathrm{nb}^{-1}$. Both events were consistent with expected backgounds from conventional hadronic and leptonic $Z$ decays. For comparison, in the same data sample, one would expect a mean of 7.6 events after the same cuts for a 44 $\mathrm{GeV}$ charged lepton. Figure 2.27 shows the distribution of visible energy versus missing transverse momentum for the OPAL data. (The signal region is indicated by solid lines).

To reduce sensitivity to assumptions concerning heavy lepton branching ratios, a second, independent search was conducted, looking for events with an isolated, energetic electron or muon, together with missing transverse momentum. The electron identification relied on a comparison of track momentum measured by the central drift chambers with energy deposited in the lead-glass calorimeter. Requirements were also imposed on the shape of the electromagnetic shower as measured by the lead-glass blocks. The muon identification relied on a comparison between extrapolated positions of drift chamber tracks and corresponding signals in either 
the hadron calorimeter strips or the outer muon barrel drift chambers. Because candidate tracks were required to be isolated by 30 degrees from other charged tracks, conventional backgrounds from misidentified hadrons were already much reduced, allowing relatively loose requirements to be made for electron and muon identification. Efficiencies were determined directly from the data itself, using primarily electron- and muon-pair events. The identification efficiency was measured to be $86 \pm 3 \%$ for electrons and $92 \pm 3 \%$ for muons with momenta greater than $5 \mathrm{GeV}$.

For events with an isolated electron or muon, two hemispheres separated by the plane perpendular to the lepton's direction were defined. If the transverse masses of the hemispheres were less than $2 \mathrm{GeV}$ and the acolinearity between the hemisphere momentum directions was less than $250 \mathrm{mrad}$, the event was classified as conventional dilepton and discarded. The remaining backgrounds come primarily from radiative production of dileptons and from two-photon production of dileptons. To remove these events, we required the acoplanarity (acolinearity in a plane transverse to the beam) be greater than $200 \mathrm{mrad}$, the total missing transverse momentum be greater than $6 \mathrm{GeV}$, and that there be no more than $5 \mathrm{GeV}$ detected in the low-angle forward detector calorimeters. At this stage, we expect $1.3 \pm 1.0$ events from conventional backgrounds, and we observe 1 event, consistent with radiative tau-pair production where the photon converted in the detector. This last event was removed by rejecting events satisfying stringent topology criteria associated with radiative lepton production. The additional topology requirements have a negligible effect on the heavy lepton detection efficiency. In the end, we have no signal events, while for comparison, a heavy lepton of $44 \mathrm{GeV}$ would be expected to produce a mean of 3.8 events for the same data sample.

Using the two searches together allows a heavy charged lepton with an associated massless neutrino to be excluded for charged masses below $44.3 \mathrm{GeV}$. Allowing for a massive neutrino partner does not change the charged mass limit for neutrino masses less than $20 \mathrm{GeV}$. For heavier neutrino masses, the selection efficiency decreases substantially for both searches, as the visible energy drops. Figure 2.28 shows the region of charged lepton masses excluded by these searches.

Gauge invariance of electromagnetism requires a massless photon, but there is no principle requiring a massless neutrino. In the Standard Model, only neutrinos are assumed to be massless fermions. In fact, it is more natural for the neutrino to be massive in GUTs. Recent LEP results show that the $Z^{0}$ line shape is fitted better with three "light" neutrinos than with four. But the fourth generation lepton with a massive neutrino is not excluded by direct search. The heavy lepton working group also conducted a search for neutral heavy leptons in the 1989 data sample [34], both through doublet production, $e^{+} e^{-} \rightarrow L^{0} \overline{L^{0}}$, where accessible masses are limited to the available beam energy, and through singlet production through mixing with a conventional neutrino, $e^{+} e^{-} \rightarrow \nu \bar{L}^{0}$ or $\bar{\nu} L^{0}$, where masses up to the total centerof-mass energy can be reached, but where production rates depend directly upon mixing strengths. In both cases, the neutral heavy lepton was assumed to decay 


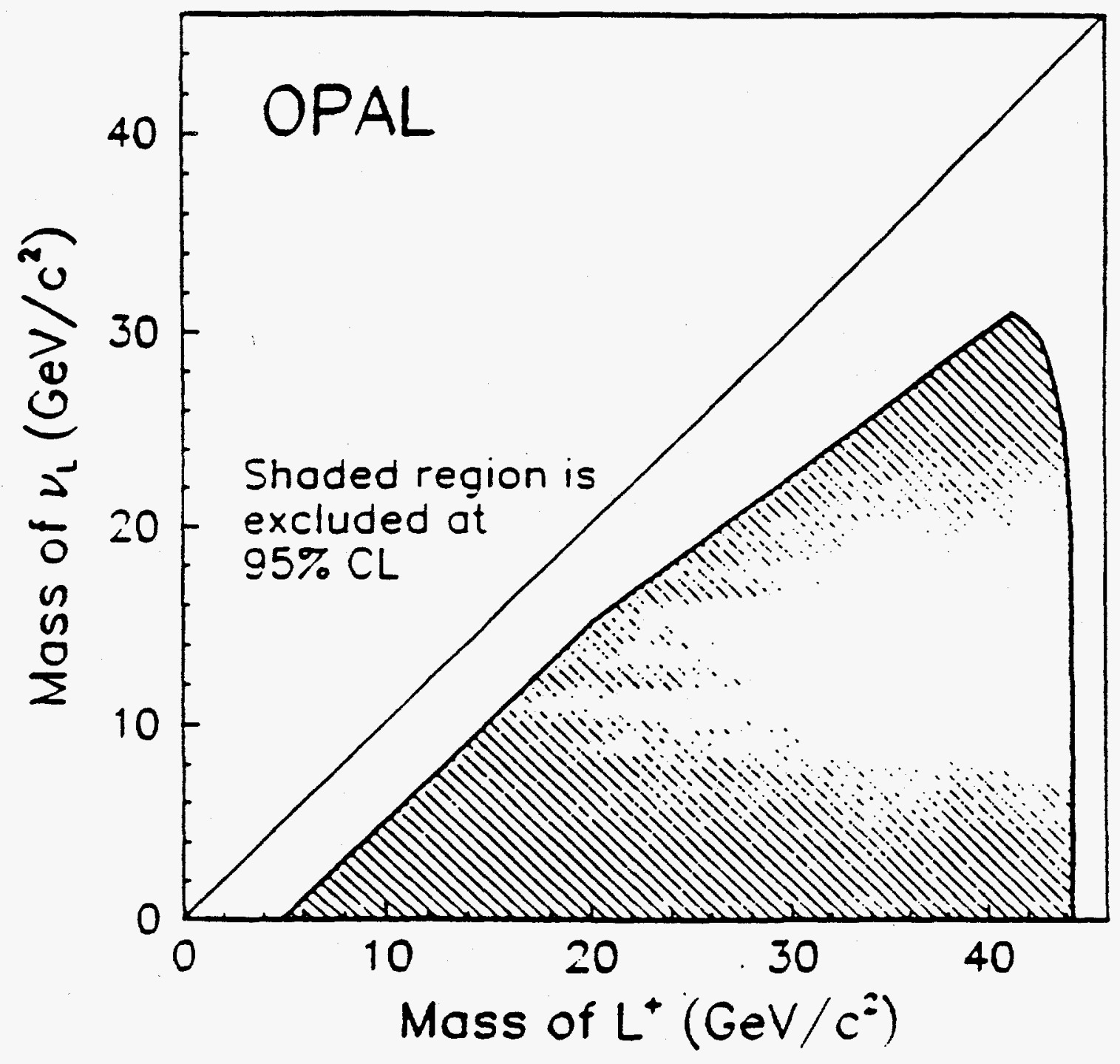

Figure 2.28: Charged lepton mass region excluded by these searches 

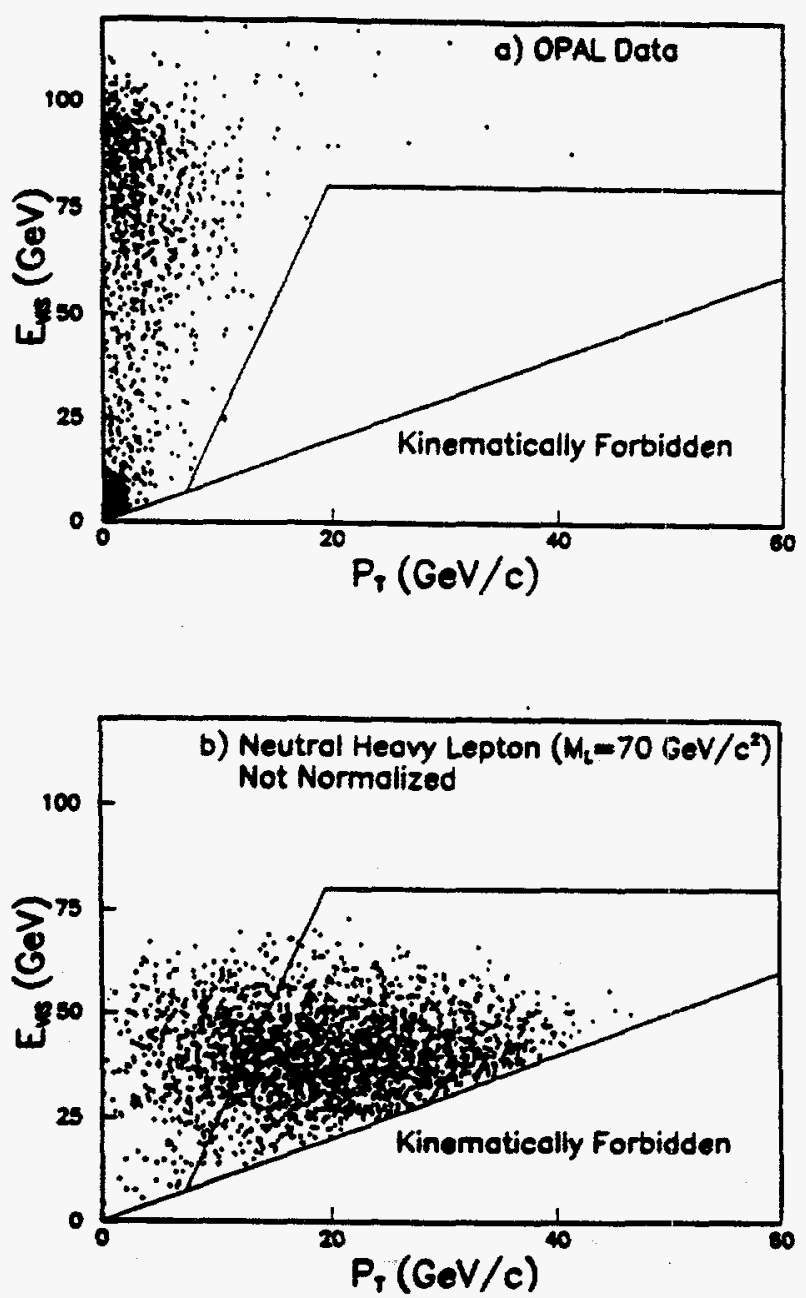

Figure 2.29: Visible energy versus transverse momentum plot for neutral heavy lepton search. The lower plot is the result of a Monte Carlo study for a lepton of $70 \mathrm{GeV}$ mass.

through mixing to conventional leptons and hadrons through either the $\mathrm{W}$ or $\mathrm{Z}$ boson: $l^{0} \rightarrow l W^{*}$ or $\nu Z^{*}$. Again, two independent searches were used.

As in the charged lepton search, the first approach looked for small visible energy and missing transverse momenta, though with slightly different requirements. Figure 2.29 which shows the allowed region in visible energy versus transverse momentum, where a tighter cut $(0.2 \mathrm{GeV})$ has been imposed on the allowed energy in a cone centered about the missing-momentum direction. To suppress various backgrounds, further requirements are introduced. Since two-photon processes are peaked in the forward-backward region, it is required that the direction of missing transverse momentum be in the barrel region. Multihadron background is reduced mostly by requiring visible energy not be larger than $30 \mathrm{GeV}$ and is further reduced by requiring the ratio of missing transverse momentum to visible energy be larger 
than 0.3. Conventional dilepton background is rejected by requiring that the thrust value be less than 0.98 . One event survived all requirements, consistent with the $1.2 \pm 0.5$ events expected from tau-pair backgrounds.

The second search required not only an isolated electron or muon, as in the charged heavy lepton search, but also another isolated track, which need not be identified as a lepton. In addition, the events were required to have at most four charged tracks in total. The remaining events are conventional dilepton and two photon processes. Since dilepton events are back-to-back and two photon processes have small acoplanarity angle, requiring both acoplanarity and acolinearity angle larger than 0.4 radians eliminates most of those backgrounds. To remove remaining backgrounds from radiative lepton-pair production where the photon converts, the transverse mass of all charged tracks other than the two isolated tracks was required to be greater than $1.5 \mathrm{GeV}$. No events survived all of these requirements.

Together these two searches permitted limits to be placed on branching ratios for producing the doublet or singlet neutral heavy leptons, with various assumptions about decay modes, as shown in Figure 2.30 Limits of a few times $10^{-4}$ were achieved for direct decay to electron or muon through $\mathrm{W}$ production, while limits on decay to taus or through $\mathrm{Z}$ production were somewhat higher. For singlet production, the limits are substantially worse for neutral lepton masses above $70 \mathrm{GeV}$, since most of the neutral lepton decays begin to resemble conventional hadronic events. Assuming that the relative branching ratios for decay through a W or $\mathrm{Z}$ are 2:1 and that mixing to one conventional lepton generation predominates over the other two, one can derive the limits on mixing angle versus neutral lepton mass shown in Figure 2.31 . For comparison, the dash-dotted limits shown are obtained from "weak-universality" limits of lower-energy experiments. As OPAL collects more data, the limits on mixing angles will improve steadily.

The Riverside group has several additional goals for this year in heavy lepton physics. One goal is to produce an update of both charged and neutral lepton searches using all of 1989 and 1990 data. The mass limits for doublet production should improve slightly, to better than $46 \mathrm{GeV}$, while the limits on mixing angles in singlet production should improve by perhaps as much as an order of magnitude, depending on integrated luminosity. The second goal is to improve the direct search for a heavy charged lepton to permit exploration of the case where both the charged lepton and its neutral partner are very heavy. A final goal of the 1990 heavy lepton analysis is to improve the sensitivity of the search for singlet neutral leptons in the high-mass region $\left(M_{L}>70 \mathrm{GeV}\right)$.

\section{Excited Leptons}

A search for excited leptons has been carried out using the full 1989 data sample. The channels considered were $e^{+} e^{-} \rightarrow l^{+} l^{-} \gamma$ and $e^{+} e^{-} \rightarrow l^{+} l^{-} \gamma \gamma$. The search assumed that the excited lepton $l^{*} \equiv e^{*}, \mu^{*}$, or $\tau^{*}$ is a spin $\frac{1}{2}$ particle and decays ex- 

$e^{+} e^{-} \rightarrow L \bar{L}$
$e^{+} e^{-} \rightarrow \nu \bar{L}$ or $\nabla L^{\circ}$

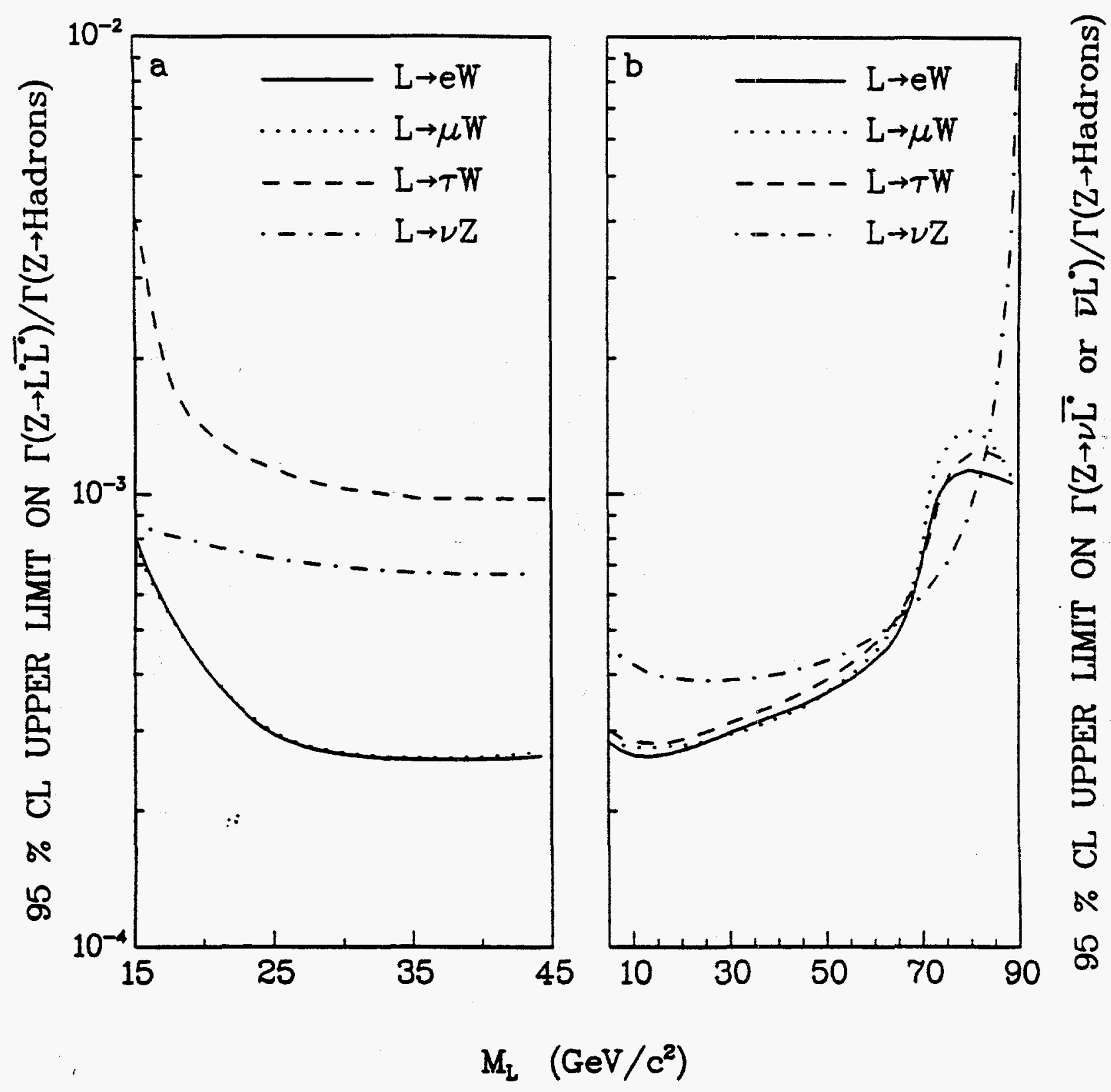

Figure 2.30: Limits on branching ratios for doublet or singlet neutral heavy leptons, for various decay modes 


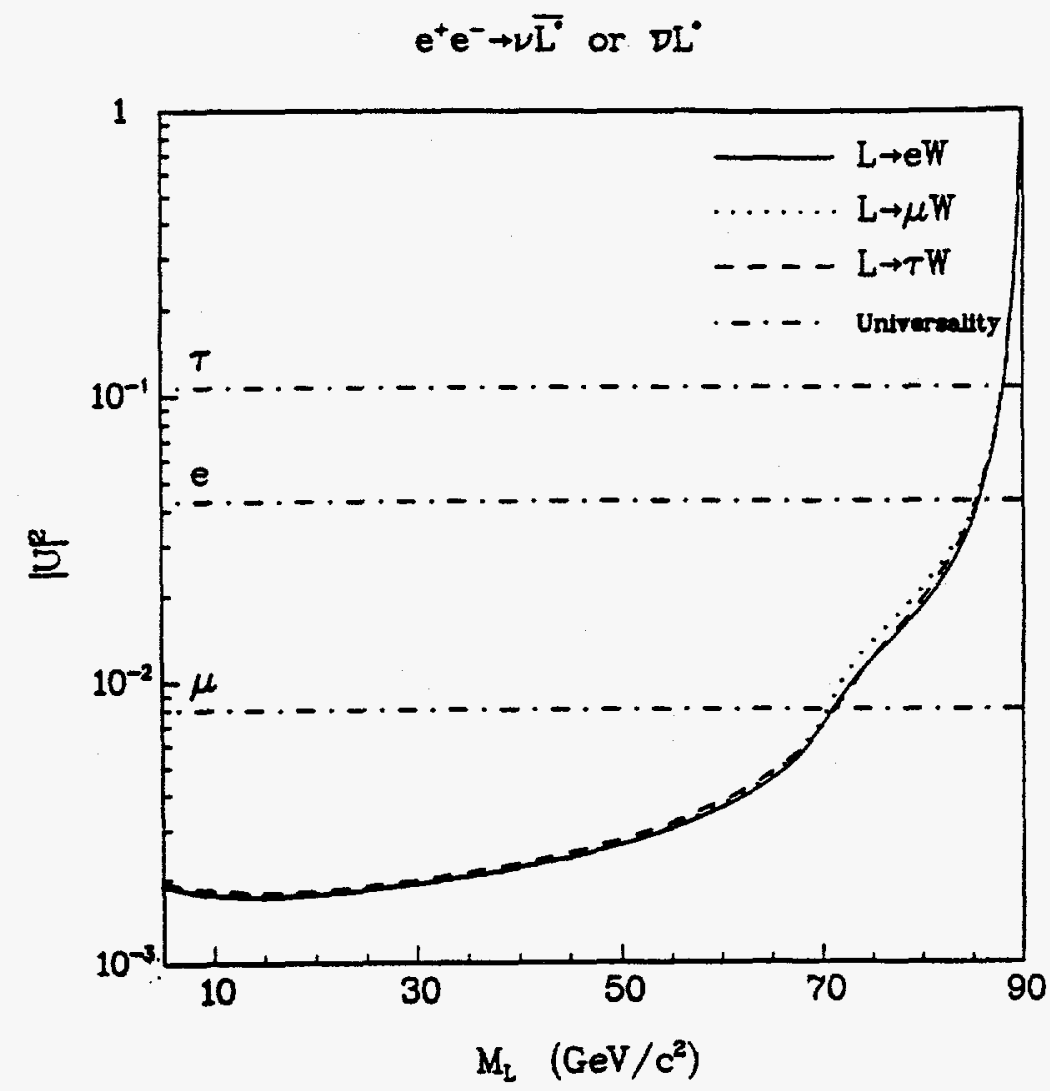

Figure 2.31: Limits on mixing angle versus neutral lepton mass

clusively through $l^{*} \rightarrow l \gamma$. In composite models the effective Lagrangian describing this decay is expressed as [35]

$$
L_{\text {eff }}=\sum_{V=\gamma, Z^{0}} \frac{e}{\Lambda} \bar{l}^{*} \sigma^{\mu \nu}\left(c_{V}-d_{V} \gamma^{\sigma}\right) l F_{\mu \nu}^{V}+h . c .
$$

Results from $g-2$ and electric dipole experiments imply that the coupling constants $c_{V}$ and $d_{V}$ are largely real and approximately equal, so the expression can be simplified to a function of one parameter $f / \Lambda$ where $\Lambda$ is the compositeness mass scale and $f$ is a reduced coupling constant. If $m_{l} \cdot<E_{e m} / 2$, the excited lepton can be pair-produced via the standard coupling and the final state will be $l^{+} l^{-} \gamma \gamma$. If $E_{c m} / 2<m_{l}<E_{c m}$, the production will occur via the couplings $\gamma l^{\prime \prime} l$ and $Z^{\circ} l^{*} l$, and the final state will be $l^{+} l^{-} \gamma$, with one $(l \gamma)$ system having the invariant mass $m_{l}^{*}$.

An exhaustive discussion of selection criteria and details of event reconstruction is provided in the published paper [36]. In summary, excited leptons have been searched for by studying the processes $e^{+} e^{-} \rightarrow e^{+} e^{-} \gamma \gamma, e^{+} e^{-} \rightarrow e^{+} e^{-} \gamma$, $e^{+} e^{-} \rightarrow\left(e^{ \pm}\right) e^{\mp} \gamma, e^{+} e^{-} \rightarrow \mu^{+} \mu^{-} \gamma \gamma, e^{+} e^{-} \rightarrow \mu^{+} \mu^{-} \gamma, e^{+} e^{-} \rightarrow \tau^{+} \tau^{-} \gamma \gamma$, and $e^{+} e^{-} \rightarrow \tau^{+} \tau^{-} \gamma$. The observed events are consistent with the Standard Model expectations and no evidence for spin $-\frac{1}{2}$ excited leptons was found. From the study of $e^{+} e^{-} \rightarrow l^{+} l^{-} \gamma \gamma$ events, lower limits on the masses were found to be $m_{l \cdot}>44.9$ $\mathrm{GeV}$ at $95 \%$ confidence level. From the study of $e^{+} e^{-} \rightarrow l^{+} l^{-} \gamma$ events, upper limits on the $l^{*} l V\left(V=\gamma, Z^{0}\right)$ couplings are set up to $l^{*}$ masses close to the $Z^{0}$ mass, as seen in Figure 2.32. 


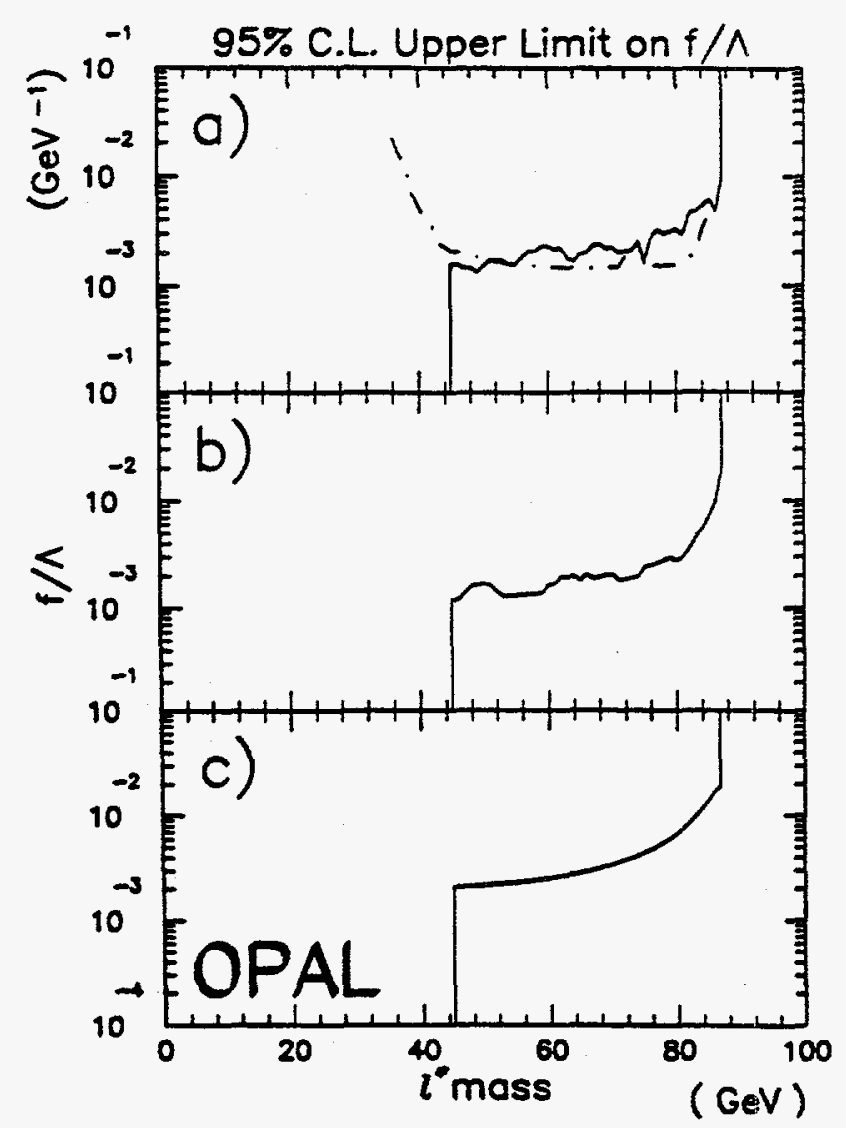

Figure 2.32: Upper limits on the coupling $f / \Lambda$ as functions of the mass of the excited leptons. Limits are for: a) $e^{*}$ from $e^{+} e^{-} \rightarrow e^{+} e^{-\gamma}$ (solid curve) and from $e^{+} e^{-} \rightarrow\left(e^{ \pm}\right) e^{\mp} \gamma$ (dot-dashed curve); b) $\mu^{*}$; and c) $\tau^{*}$.

\section{Supersymmetric Particles}

We have carried out a search for $Z^{0}$ decays into pairs of massive particles decaying promptly to conventional quarks or leptons and undetected neutrino-like particles, using data corresponding to an integrated luminosity of $0.55 \mathrm{pb}^{-1}$. Though other sources of physics can produce similar signatures, the main motivation for this search is supersymmetric models: assuming that the photino $\tilde{\gamma}$ is the lightest stable SUSY particle, the partners of the charged slepton $\tilde{l}$ could decay promptly to $l$ and $\tilde{\gamma}$. The partners of the charged bosons $\left(W^{ \pm}, H^{ \pm}\right)$, called "charginos" and denoted by $\tilde{\chi}^{ \pm}$, are expected to have both leptonic $\left(\tilde{\chi}^{ \pm} \rightarrow l \nu \tilde{\gamma}\right)$ and hadronic $\left(\tilde{\chi}^{ \pm} \rightarrow q \bar{q} \tilde{\gamma}\right)$ decay modes, whose branching ratios depend on the unknown mixing between the charginos.

Among backgrounds from conventional sources, the missing momentum vector points either along the beam axis (for initial state radiation or two-photon events) or along the direction of the detected lepton (for $\tau$ decays). We therefore searched for acoplanar events, or those in which the observed particles or jets are acolinear in the plane perpendicular to the beam axis. Figure 2.33 shows the $95 \%$ confidence level contour plots of mass limits of the sleptons as functions of the $\tilde{\gamma}$ mass.

For the charginos, whose decay involves the $\tilde{\gamma}$ as the lightest supersymmetric particle (LSP), the analysis is substantially the same. The excluded region is presented in Figure 2.34 as a contour plot of the hadronic branching ratio of the $\tilde{\chi}^{ \pm}$ versus its mass. In the plot, the excluded regions from an analysis based on electromagnetic clusters are those interior to curves a) and b), while the regions excluded 

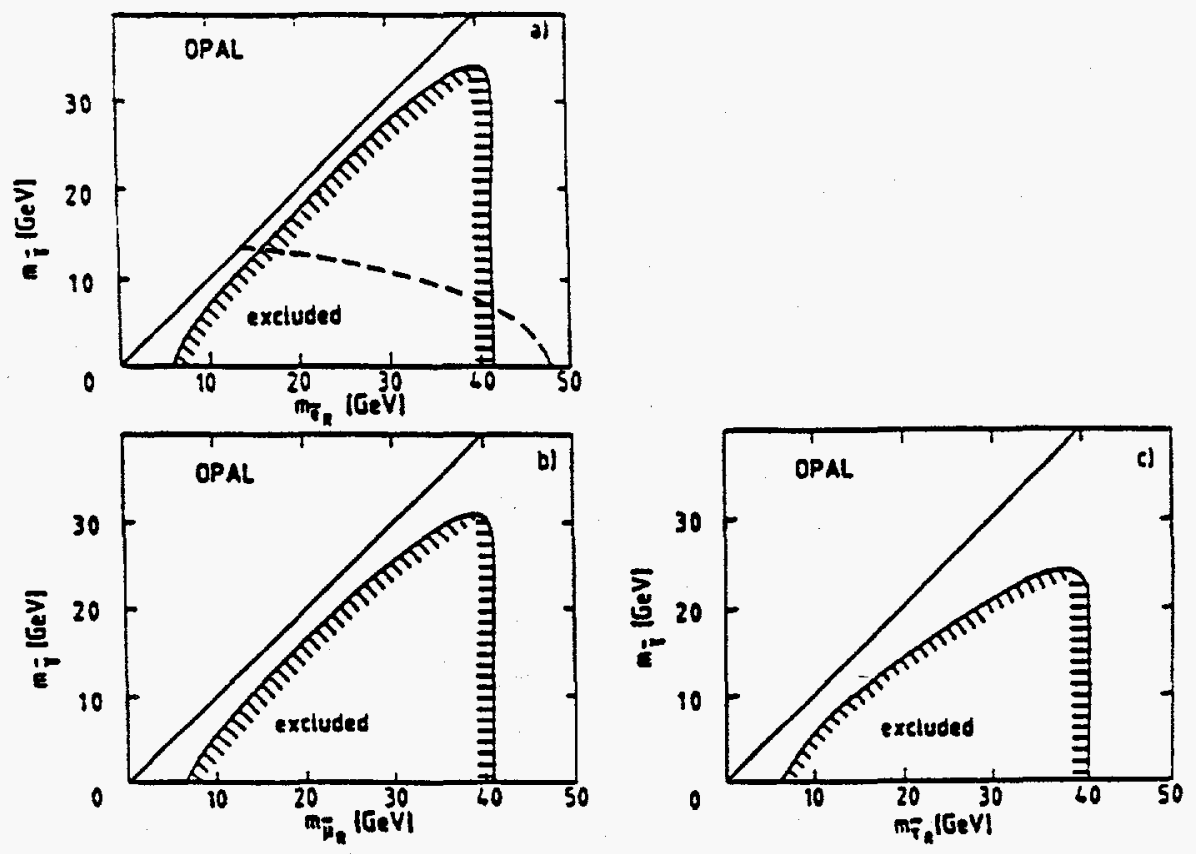

Figure 2.33: Excluded regions in $m_{\tilde{\gamma}} v s . m_{\tilde{l}}$ for a) $\tilde{e}$, b) $\tilde{\mu}$, and c) $\tilde{\tau}$.

by the hadron jet analysis are those interior to curves c) and d).

The supersymmetric partners of the neutral gauge bosons are the "neutralinos". The $Z^{0}$ is expected to couple strongly to pairs of neutralinos, so LEP is a good place to search for them. Now the lightest neutralino $\chi$ is assumed to be the LSP. It is stable from R-parity conservation and so becomes invisible. The heavier neutralinos, denoted by $\chi^{\prime}$ can decay to $\chi$ through $\chi^{\prime} \rightarrow \chi f \bar{f}$ or $\chi^{\prime} \rightarrow \chi \gamma$. (We assume that the masses of $h^{0}$ and $\tilde{\chi}^{ \pm}$are sufficiently large that $\chi^{\prime} \rightarrow \chi h^{0}$ and $\chi^{\prime} \rightarrow \bar{\chi}^{ \pm} f \bar{f}$ are kinematically forbidden.)

The cleanest signature of the process $e^{+} e^{-} \rightarrow \chi \chi^{\prime} \rightarrow \chi \chi f \bar{f}$ is an event topology of large missing momentum and large acoplanarity due to the two undetected neutralinos. Hence we searched for acoplanar two-jet events with small visible energy. Since a jet could consist of a single charged particle, lepton-pair events were classified as two-jet events. Thus we treated quarks. and leptons inclusively for the final state fermion pair. Details of the cuts and selection will be available in preprint form shortly. For the $\chi \chi^{\prime} \rightarrow \chi \chi \gamma$ mode, since the neutralino is invisible, the event signature is the same as that of the single photon events, with the exception that one can put a high cut on the photon energy, in this case $10 \mathrm{GeV}$. The result gives upper limits on the branching ratios for $\chi^{\prime} \rightarrow \chi f \bar{f}$ and for $\chi^{\prime} \rightarrow \chi \gamma$, which can be combined to give an upper limit on the branching ration $\mathrm{BR}\left(Z^{0} \rightarrow \chi \chi^{\prime}\right)$ which is independent of any assumption about the relative strengths of the $\chi^{\prime} \rightarrow \chi f \bar{f}$ and $\chi^{\prime} \rightarrow \chi \gamma$ decays. This branching ratio is shown in Figure 2.35 plotted as a function of $m_{\chi^{\prime}}$ versus $m_{\chi}$. 


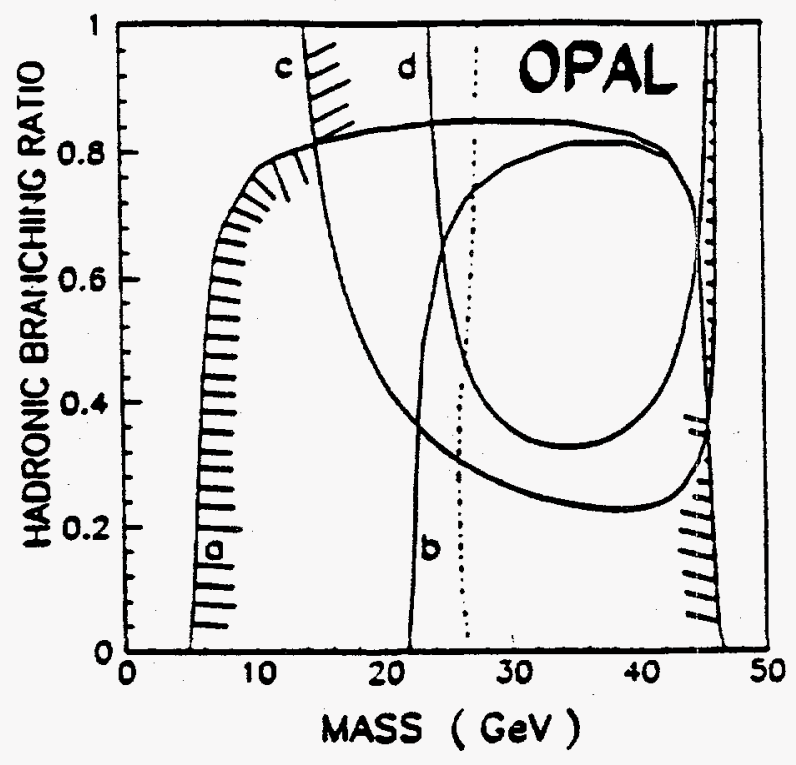

Figure 2.34: Excluded regions for $\tilde{\chi}^{ \pm}$. Curves a) and c) are for $m_{\tilde{\gamma}}=0$, and curves b) and d) for $m_{\tilde{\gamma}}=20 \mathrm{GeV}$.

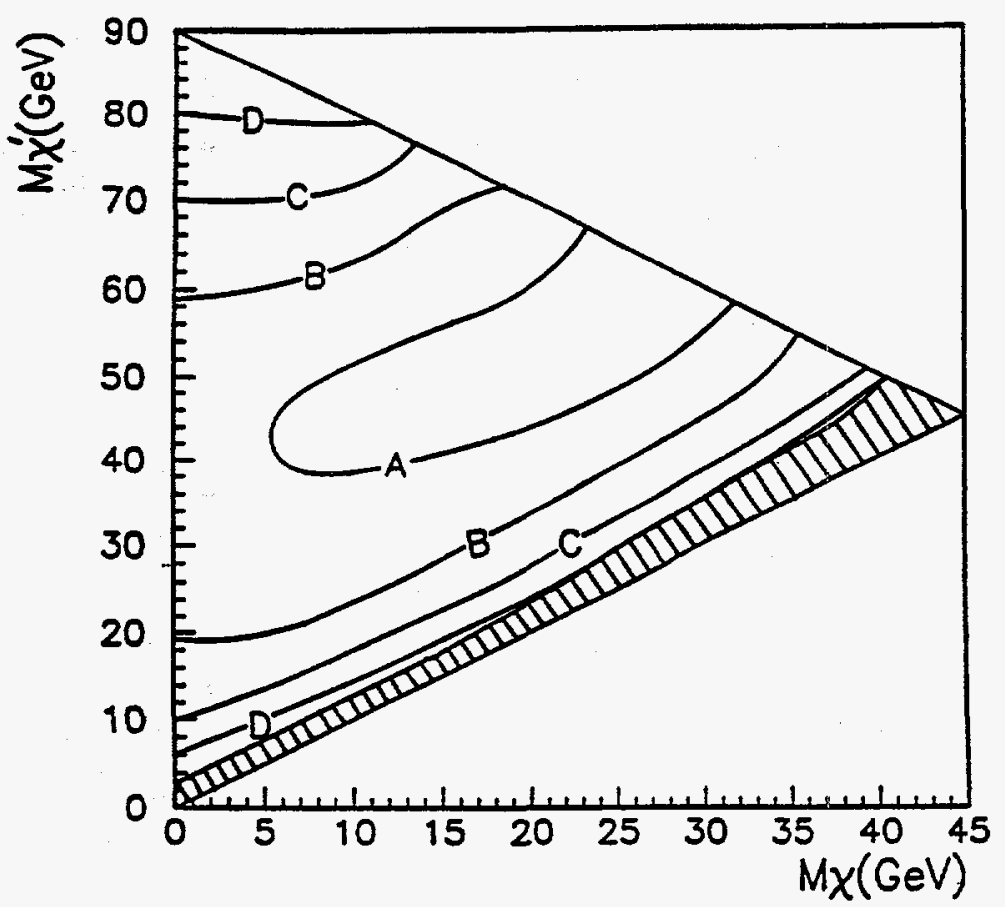

Figure 2.35: Upper limit on the branching ratio $\mathrm{BR}\left(Z^{0} \rightarrow \chi \chi^{\prime}\right)$ at $95 \%$ confidence level. Curves A, B, C, and D indicate the contours of $1.5,2.0,3.0$, and $5.0 \times 10^{-4}$. 


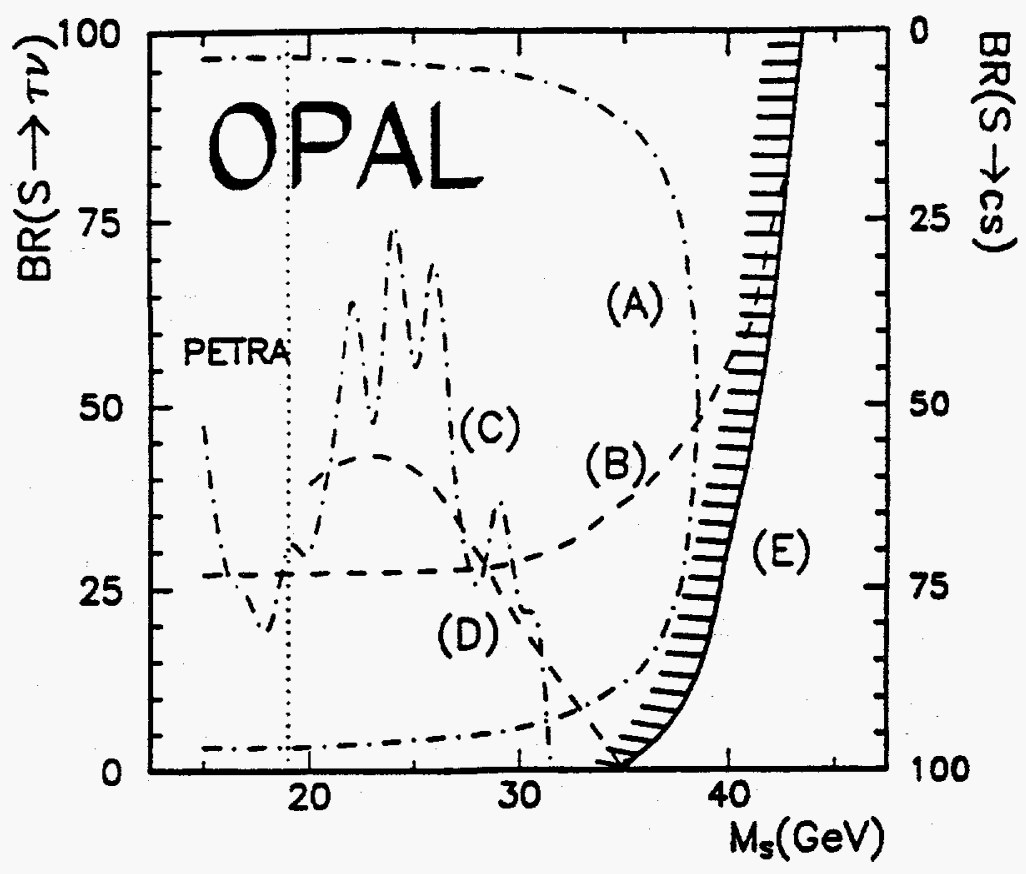

Figure 2.36: Limits for $\mathrm{BR}\left(S^{ \pm} \rightarrow c s\right)$ versus the $S^{ \pm}$mass.

\section{Charged Scalar Particles}

We have conducted a general search for scalar particles $S^{ \pm}$based on the study of the channels $e^{+} e^{-} \rightarrow S^{+} S^{-} \rightarrow(c s)(\tau \nu),(c \bar{s})(\bar{c} s)$, and $(\tau \bar{\nu})(\bar{\tau} \nu)$ [37]. Natural candidates for such states are technipions and charged Higgs bosons. While the production cross section $e^{+} e^{-} \rightarrow S^{+} S^{-}$is described by a simple formula, the branching ratios of the $S^{ \pm}$are model dependent: the dominant decay modes are expected to be $(\tau \bar{\nu})$ and $(\bar{c} s)$, while $(c \bar{b})$ is suppressed.

Our analysis does not depend explicitly on the quark flavor. The search for the (cs)(TV) channel is carried out on a sample of about 22000 hadronic decays, looking for the topology of one isolated charged particle; with or without neutrals, recoiling against two hadronic jets. The search for the $(\tau \bar{\nu})(\bar{\tau} \nu)$ channel is carried out using the entire 1989 data sample, looking for events with an acoplanar $\tau$ pair due to the large missing momentum carried away by the neutrinos. The search for the $(c \bar{s})(\bar{c} s)$ channel is based on a 4-jet analysis under the constraint that $S^{+}$and $S^{-}$ have the same mass and the same velocity. The results are plotted as functions of $\operatorname{BR}\left(S^{ \pm} \rightarrow c s\right)=1-\operatorname{BR}\left(S^{ \pm} \rightarrow \nu \tau\right)$ versus the mass of the $S^{ \pm}$in Figure 2.36, where the curves (A), (B), (C), and (D) represent the limits obtained from the analysis of $(c s)(\tau \nu),(\tau \bar{\nu})(\bar{\tau} \nu),(c \bar{s})(\bar{c} s)$ low mass, and $(c \bar{s})(\bar{c} s)$ high mass channels, respectively. Curve $(E)$ is the combined limit. All limits are at the $95 \%$ confidence level.

\section{Minimal Standard Model Higgs}

Spontaneous symmetry breaking is the foundation of the Standard Model of weak and electromagnetic interactions [38]. In local gauge invariant theories, spontaneous 
symmetry breaking requires the existence of one or more scalar particles, the Higgs bosons [39]. However, while the Standard Model has been successful in describing many processes with a high degree of accuracy, these particles have not been observed. In the minimal Standard Model (MSM) one complex doublet of Higgs fields is introduced, which results in a single scalar Higgs boson, $H^{0}$ with a mass that is not specified by the theory.

The present analysis has been performed using all data from $1989\left(1.244 \mathrm{pb}^{-1}\right)$ and runs 8 through 12 from $1990\left(2.13 \mathrm{pb}^{-1}\right)$. Two types of search were undertaken: the missing energy search directed towards events of the type $e^{+} e^{-} \rightarrow Z^{0^{*}} H^{0}$ with $Z^{0^{*}} \rightarrow \nu \bar{\nu}$ and $H^{0} \rightarrow q \bar{q}$, and the dilepton search looking for the presence of two isolated energetic leptons from $Z^{0} \rightarrow\left(e^{+} e^{-}\right.$or $\left.\mu^{+} \mu^{-}\right) H^{0}$. The essence of the missing energy search is the definition of a "forward cone" of $75^{\circ}$ half angle which contains more than $70 \%$ of the visible energy of the event. For Higgs events the corresponding "backward cone" of $60^{\circ}$ half angle should contain less than $2.5 \mathrm{GeV}$. The results of the two types of analysis are combined to give the final result. Since no events survive the cuts, the $95 \%$ confidence level mass limit is obtained by seeing where the predicted number of events at a given mass intersects the 3-event level. This is shown in Figure 2.37 where plot a) shows the results of the two types of analysis for the 1989 data, while plot b) gives the combined result for the high mass region for both the 89 and 90 data and shows that a mass limit of $34 \mathrm{GeV}$ has been achieved. The lower limit of $3 \mathrm{GeV}$ is set somewhat arbitrarily by an estimate of the reliability of the Monte Carlo simulation of backgrounds.

\section{Minimal Supersymmetric Model Higgs}

In a more general case, two Higgs doublets are considered and the Higgs sector contains five physical Higgs bosons. A popular theory with such a Higgs sector is supersymmetry (SUSY). In the minimal SUSY Model (MSSM) one of the Higgs doublets couples to up-type fermions only, while the other couples only to downtype fermions, thus preventing flavor-changing neutral currents. Of the two neutral scalar Higgs bosons in this model, one $\left(h^{0}\right)$ must be lighter than the $Z^{0}$, while the other must be heavier than the $Z^{0}$. There is also a charged Higgs pair which has to be heavier that the W, and a neutral CP-odd or pseudoscalar Higgs $\left(A^{0}\right)$ which has to be heavier than the $h^{0}$ but can be lighter than the $Z^{0}$ and might therefore also be accessible to experiments running near the $Z^{0}$ resonance. In this model, two independent parameters are needed to specify the Higgs sector. There are two common choices for these parameters: i) $\tan \beta=v_{2} / v_{1}$, the ratio of the vacuum expectation values of the doublets, and $m_{h^{0}}$, the mass of the lightest Higgs; and ii) $m_{h^{0}}$, and $m_{A^{0}}$, the mass of the pseudoscalar Higgs.

The search for the MSM Higgs described previously can be applied directly to the mode $Z^{0} \rightarrow Z^{0^{*}} h^{0}$ to exclude a significant region of the $m_{h^{0}}, m_{A^{0}}$ parameter space. Other regions of the parameter space have been excluded using the decay $Z^{0} \rightarrow$ 

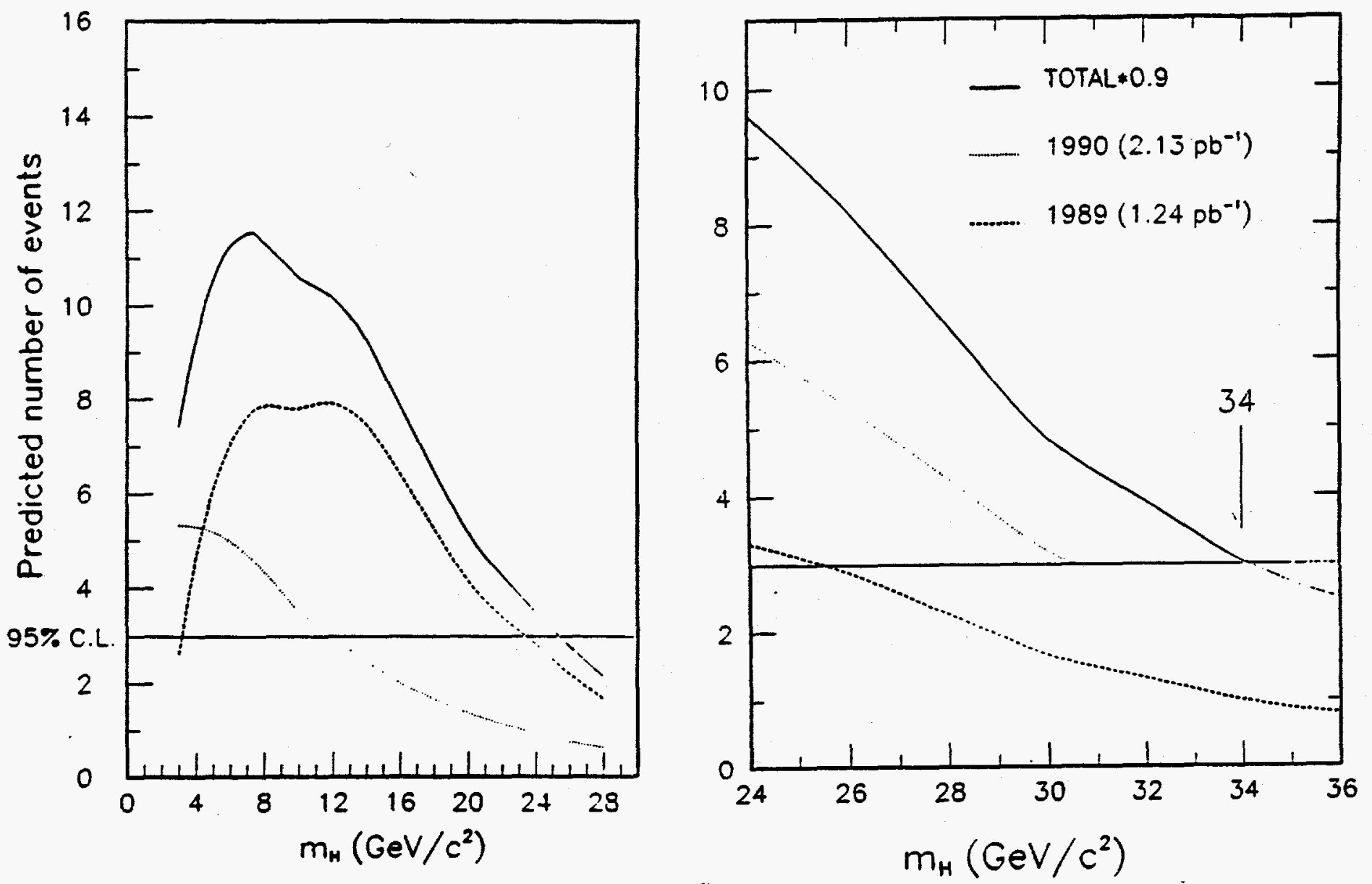

Figure 2.37: a) for 1989 data only showing the expected number of events as a function of Higgs mass for the missing energy channel (dashed line) and for the dilepton channel (dotted line), with the total number given by the solid line. b) for the high mass portion of showing the combined limit of $34 \mathrm{GeV}$ from 1989 and 1990 data. 


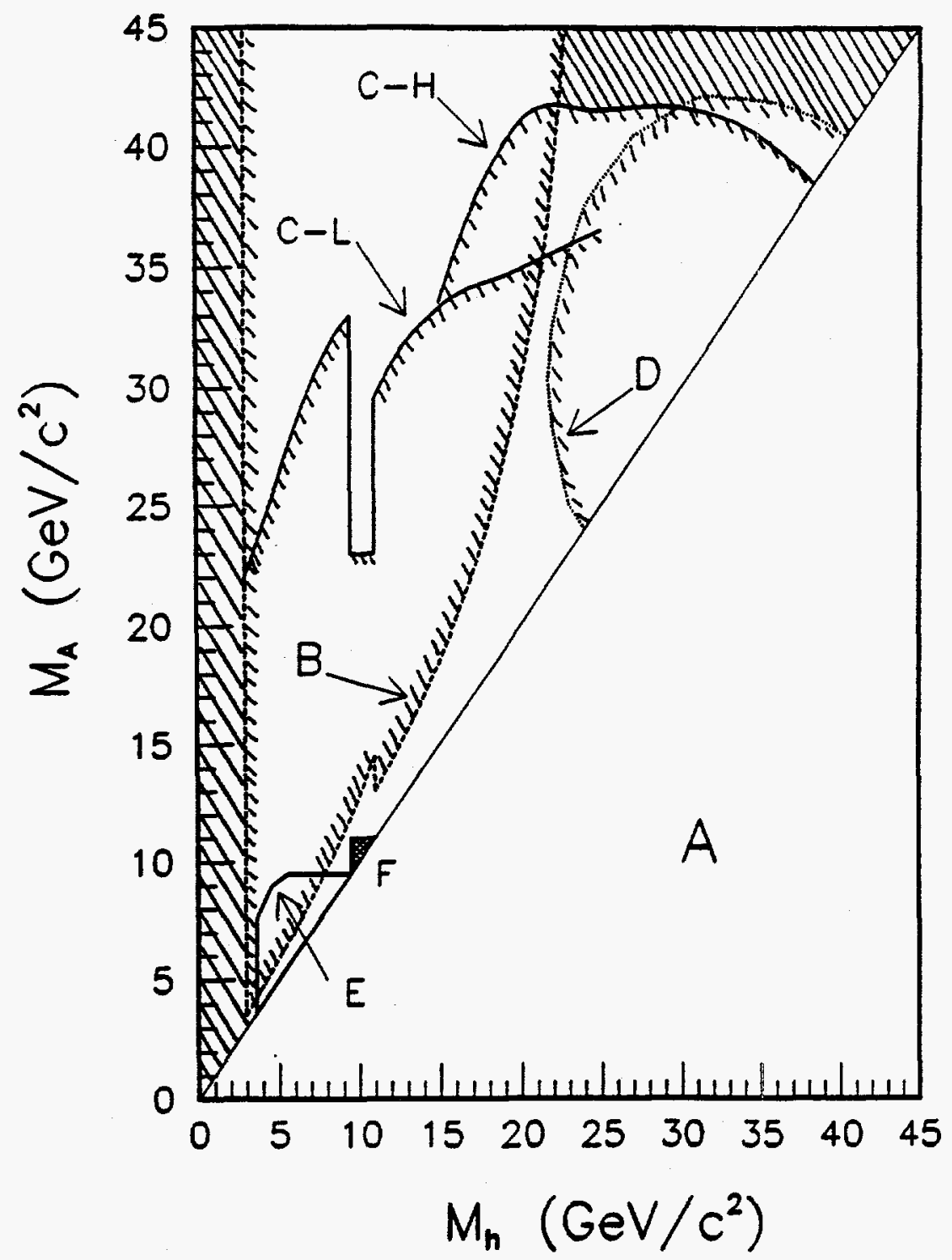

Figure 2.38: The $m_{h^{0}}, m_{A^{0}}$ parameter space

$h^{0} A^{0}$, where the Higgs bosons could be identified by their decays into $c \bar{c}, b \bar{b}$, and $\tau^{+} \tau^{-}$. These processes must be examined separately for the assumptions $\tan \beta>1$ (where $A^{0} \rightarrow b \bar{b}, \tau^{+} \tau^{-}$mostly) and $\tan \beta<1$ (where $A^{0} \rightarrow c \bar{c}$ predominantly).

The $m_{h^{0}}, m_{A^{0}}$ parameter space is shown in Figure 2.38 where each letter corresponds to a dedicated analysis: region $A$ is excluded by theory: $m_{A^{0}}>m_{h^{0}}$; region $B$ is excluded by the MSM search discussed above; region $C$ and D are excluded by a search for $e^{+} e^{-} \rightarrow h^{0} A^{0} \rightarrow \tau^{+} \tau^{-}$jet jet; region $C$ is excluded in an analysis tuned to low Higgs masses (from $b \bar{b}$ threshold to $20 \mathrm{GeV}$ ), and region $D$ is excluded in an analysis tuned to high Higgs masses (above $15 \mathrm{GeV}$ ); in the region from 9.1 to $11 \mathrm{GeV}$ where one of the Higgs masses overlaps with the $\chi_{b}$ resonant states, the hadronic branching ratio is unknown, which reduces the sensitivity and explains the dip in curve $\mathrm{C}$; if both Higgs masses were in this mass range (region F), we would not be able to exclude their existence; a search for $e^{+} e^{-} \rightarrow h^{0} A^{0} \rightarrow \tau^{+} \tau^{-} \tau^{+} \tau^{-}$ excludes region $E$. 


\section{Very Light Standard Model Higgs}

The OPAL detector has been used to set an upper limit on the decay of the Higgs boson to $e^{+} e^{-}$in the reaction $e^{+} e^{-} \rightarrow Z^{0} \rightarrow Z^{0^{*}} H^{0}$ as a function of the mass of the Higgs. In the context of the Standard Model, this study rules out a Higgs mass between $40 \mathrm{MeV}$ and the threshold for decay into two muons. The limit does not extend below $40 \mathrm{MeV}$ because as the Higgs mass decreases, an increasing proportion of the bosons are predicted to decay outside the detector volume. However, combining this analysis with that for unobserved Higgs decay, such as when the Higgs decays outside the detector, the Standard Model Higgs is ruled out for all masses up to the muon threshold.

Only the $Z^{0^{*}} \rightarrow \nu \bar{\nu}$ channel was used in this analysis to avoid the complications of radiative backgrounds present in the $Z^{0^{*}} \rightarrow l^{+} l^{-}$and $Z^{0^{*}} \rightarrow q \bar{q}$ channels. A characteristic signature of the $H^{0} \rightarrow e^{+} e^{-}, Z^{0^{*}} \rightarrow \nu \bar{\nu}$ channel is the presence of isolated energy deposition in the electromagnetic calorimeter and hits in the central tracking detector. The limit was determined by searching for events having all electromagnetic activity confined to a $30^{\circ}$ half angle cone with the cone axis between $-0.75<\cos \theta<0.75$ and a track with four or more central detector hits within the cone and no other activity in the detector.

The process $Z^{0^{*}} \rightarrow \nu \bar{\nu}, H^{0} \rightarrow \gamma \gamma$, which is suppressed in the minimal Standard Model for Higgs masses above $2 m_{e}$, can become significant in modest extensions to the Model. Very large enhancements occur, for example, in models containing more than three generations [40]. Limits on such extensions to the Standard Model have been addressed in the analysis by lifting the tracking requirement used in the search for $Z^{0^{*}} \rightarrow \nu \bar{\nu}, H^{0} \rightarrow e^{+} e^{-}$. Any limits set on the basis of such a search apply to both channels and therefore enable a limit to be set which is independent of models that enhance the $H^{0} \rightarrow \gamma \gamma$ decay mode. The combined limit obtained by this analysis is shown in Figure 2.39. 


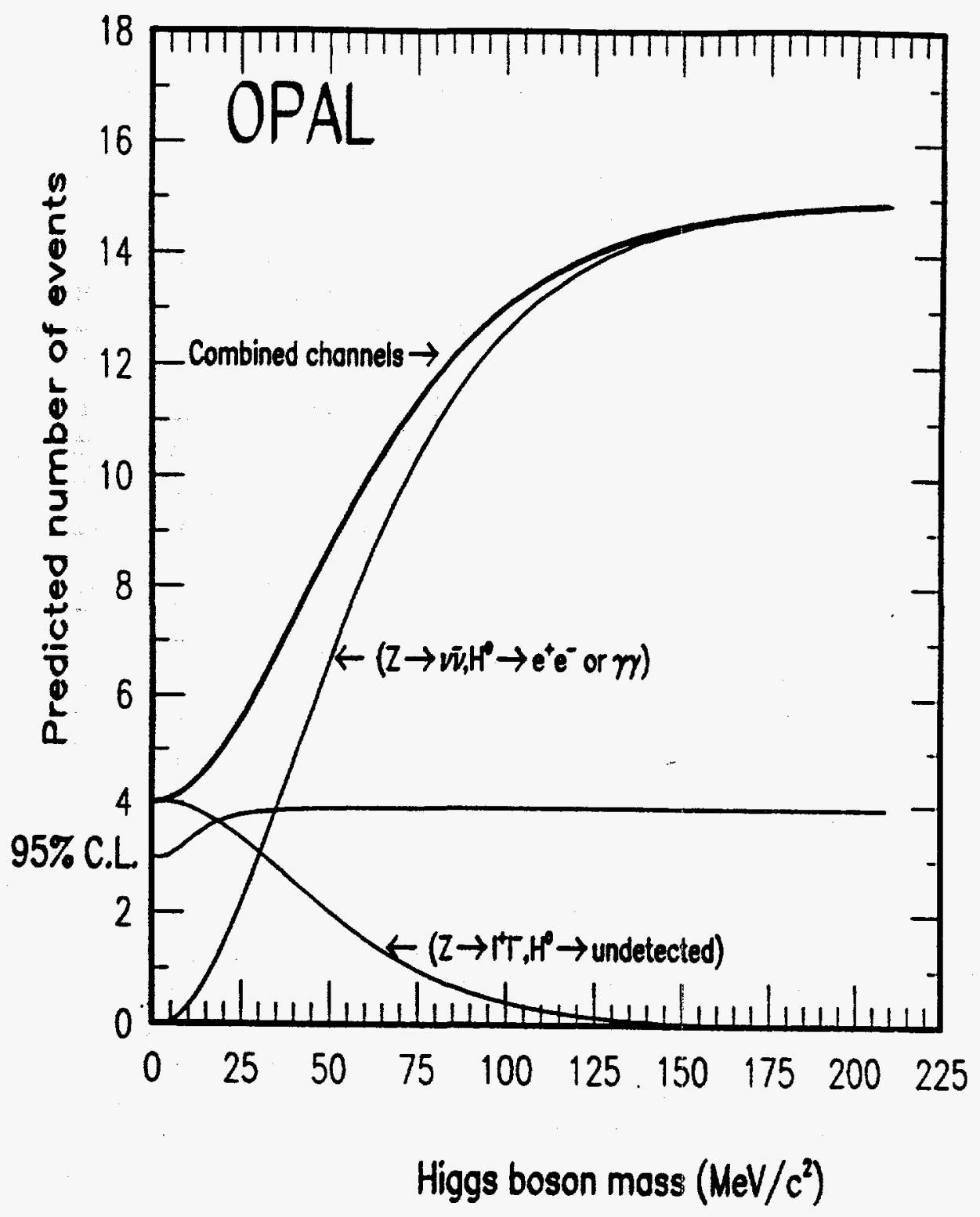

Figure 2.39: Predicted numbers of events versus Higgs mass in the low mass region. 


\subsubsection{Physics at LEP II}

LEP begins its energy upgrade program this year by introducing several superconducting RF cavities in the ring. It is planned that when all cavities are installed, LEP will be able to reach a top c.m. energy of about $210 \mathrm{GeV}$, with a design luminosity of $3 \times 10^{31} \mathrm{~cm}^{-2} \mathrm{sec}^{-1}$. We expect an integrated luminosity of between 200 and $500 \mathrm{pb}^{-1}$ per year of operation. When this is achieved, LEP II will provide us with rich and unique physics opportunities.

$e^{+} e^{-} \rightarrow W^{+} W^{-}$and the 3-boson coupling

The principal mechanisms responsible for W-pair production in high energy $e^{+} e^{-}$ collisions are the t-channel exchange of a fermion and the s-channel exchange of a vector boson, as depicted in Figure 2.40 . In the Standard Model, the large

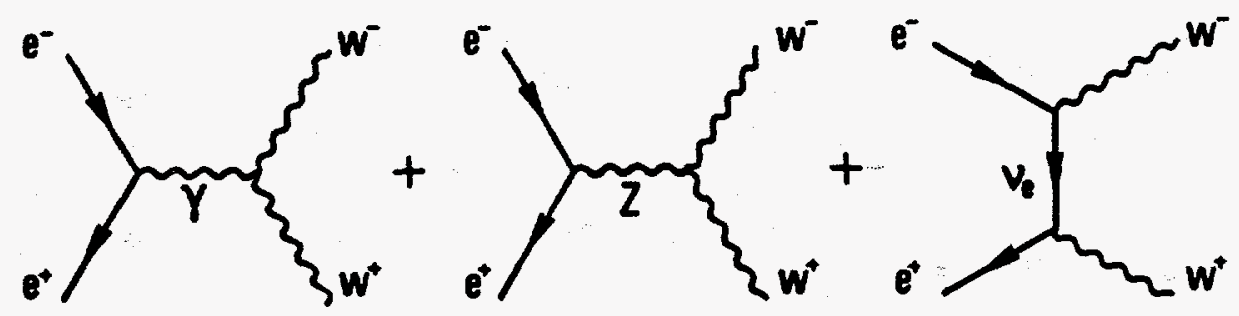

Figure 2.40: t- and s-channel diagrams contributing to $e^{+} e^{-} \rightarrow W^{+} W^{-}$in the Standard Model

cancellations between the amplitudes from these processes keep the cross section well behaved as the energy increases and prevent it from violating the unitarity bound at high energies.

At LEP II, it will be possible to determine experimentally the couplings of the three gauge bosons WW $\gamma$ and WWZ. This will be a crucial test of the Standard Model and should point to "new physics."

The most general WWV vertex $\Gamma_{V}^{\alpha \beta \mu}$ is described in terms of $2 \times 7$ independent form factors $f_{i}^{V}[41]$

$$
\begin{aligned}
& \Gamma_{V}^{\alpha \beta \mu}(q, \bar{q}, P)=f_{1}^{V}(q-\bar{q})^{\mu} g^{\alpha \beta}-\left(f_{2}^{V} / m_{W}^{2}\right)(q-\bar{q})^{\mu} P^{\alpha} P^{\beta} \\
& +f_{3}^{V}\left(P^{\alpha} g^{\mu \beta}-P^{\beta} g^{\mu \alpha}\right)+i f_{4}^{V}\left(P^{\alpha} g^{\mu \beta}+P^{\beta} g^{\mu \alpha}\right) \\
& +i f_{5}^{V} \epsilon^{\mu \alpha \beta \rho}(q-\bar{q})_{\rho}-f_{6}^{V} \epsilon^{\mu \alpha \beta \rho} P_{\rho} \\
& -\left(f_{7}^{V} / m_{W}^{2}\right)(q-\bar{q})^{\mu} \epsilon^{\alpha \beta_{\rho \sigma}} P_{\rho}(q-\bar{q})_{\sigma} \text {, }
\end{aligned}
$$




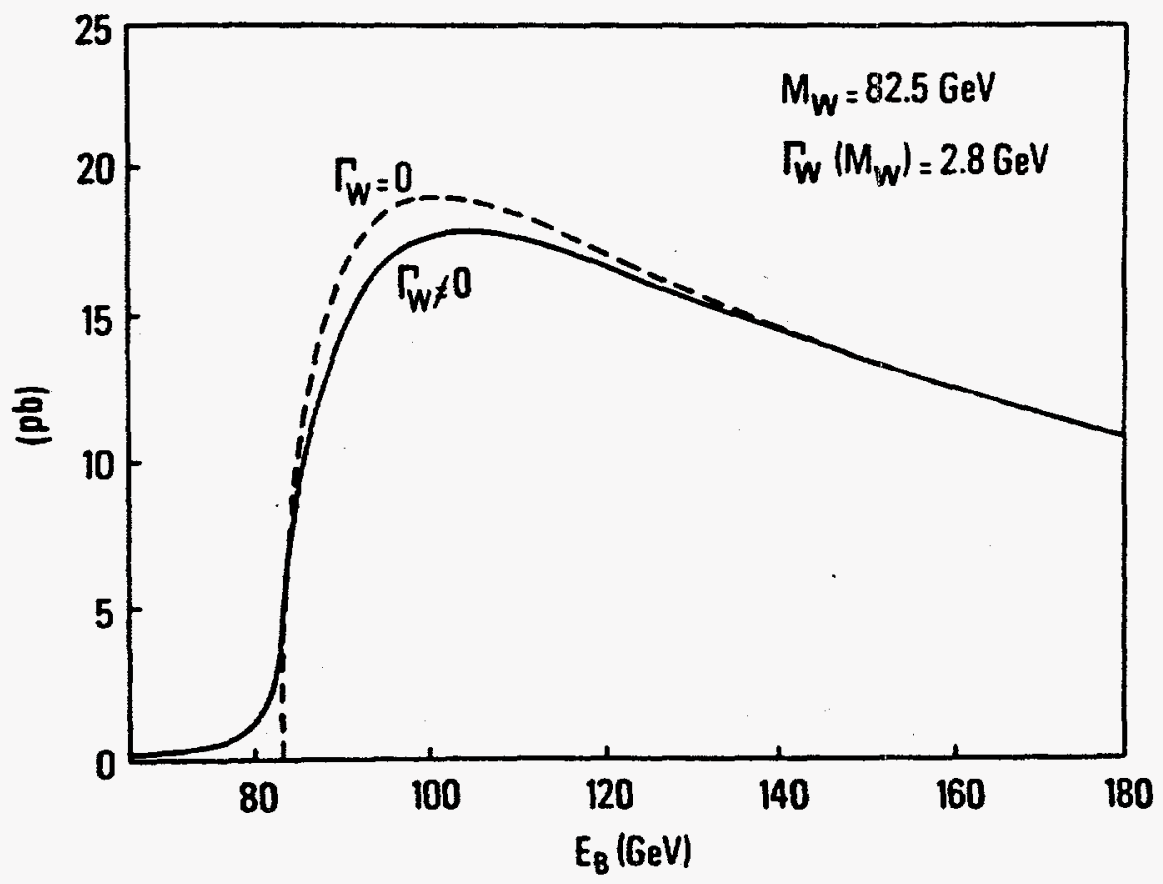

Figure 2.41: Cross section of $e^{+} e^{-} \rightarrow W^{+} W^{-}$

for $\mathrm{V}=\gamma, \mathrm{Z}$ and $g_{W W \gamma}=-e, g_{W W Z}=-e \cot \theta_{W}$. While the form factors $f_{i}^{V}$ for $i=1,2,3$ characterize couplings which are separately $P$ and $C$ conserving, for $i=4,6,7$ they correspond to CP-violating ones, and for $i=5$ to couplings separately violating $\mathrm{P}$ and $\mathrm{C}$. For $i=1,2,3$ they may be expressed in terms of the more familiar quantities $\kappa_{V}$ and $\lambda_{V}$ (for $\left.g_{1}^{Z}=G_{1}^{\prime}=1\right)$ :

$$
\begin{aligned}
& f_{1}^{V}=1+\left(s / 2 m_{W}^{2}\right) \lambda_{V}, \\
& f_{2}^{V}=\lambda_{V}, \quad V=\gamma, Z, \\
& f_{3}^{V}=1+\kappa_{V}+\lambda_{V},
\end{aligned}
$$

which in turn are directly related to the $\mathrm{W}$ magnetic dipole and electric quadrupole moments

$$
\begin{aligned}
& \mu_{W}=e\left(1+\kappa_{\gamma}+\lambda_{\gamma}\right) / 2 m_{W} \\
& Q_{W}=-e\left(\kappa_{\gamma}-\lambda_{\gamma}\right) / m_{W}^{2} .
\end{aligned}
$$

In the Standard Model all the form factors $f_{i}^{V}$ vanish at tree level except for $f_{1}^{V}=1$ or $\lambda_{V}=0$, and $f_{3}^{V}=2$ or $\kappa_{V}=1$. Once the Standard Model constraints have been relaxed, there are a large number of free parameters to be tested for.

The cross section of $e^{+} e^{-} \rightarrow W^{+} W^{-}$, assuming Standard Model couplings, is shown in Figure 2.41 . It peaks at $\sqrt{s} \approx 200 \mathrm{GeV}$ with a cross section of approximately $17 \mathrm{pb}$. A total integrated luminosity of $500 \mathrm{pb}^{-1}$ can be accumulated in one to two years of running at LEP II producing about 8500 events [42]. This sample will be divided into three different final state configurations depending on the decay of the W: 


$$
\begin{array}{lr}
2 \text { leptons + missing } \vec{p} & 700 \text { events } \\
1 \text { lepton }+ \text { hadrons }+ \text { missing } \vec{p} & 3400 \text { events } \\
\text { hadrons } & 4400 \text { events }
\end{array}
$$

From the analysis of this sample of events, we will be able to determine $\lambda_{V}$ or $\kappa_{V}$ to an accuracy of about $30 \%$ [43].

\section{The Measurement of the Mass of $W^{ \pm}$}

The mass of the $\mathrm{W}$ boson is one of the most important parameters in the Standard Model. It is among the experimentally measurable quantities which provide stringent tests of the electroweak theory beyond the tree level. As is well known, the radiative corrections calculated at the one-loop level include effects due to the top quark, whose mass is yet unknown. The precision measurement of the mass of W can place very tight limits on the mass of the top quark. It can also provide information on the scale of new physics beyond the Standard Model. At LEP II the mass of $W^{ \pm}$can be measured in several different ways [44]. The method to be used will depend on the amount of data taken at the c.m. energy chosen, and the precision that can be achieved ranges between $100 \mathrm{MeV}$ to $300 \mathrm{MeV}$ [45].

(a) The cross section near the $\mathrm{W}$-pair production threshold is sensitive to the mass of the W. By measuring the cross section at four or five energies below and above the $\mathrm{W}$ mass, it is possible to achieve a precision of about $150 \mathrm{MeV}$ after the first year or running. The systematic uncertainties depend on the knowledge of the theoretical cross section calculations, the beam energy, and the energy dependence of the background. After three years of operation and an accumulated $1.0 \mathrm{fb}^{-1}$ of data, we expect to achieve a precision of about $100 \mathrm{MeV}$. The method is unique to $e^{+} e^{-}$collisions.

(b) The mass of the $\mathrm{W}$ can be determined from reconstruction of the decay products. This can be accomplished using either hadronic or leptonic decays. In the hadronic decay, the invariant mass of the two jets from the $W$ decay can be reconstructed. Since the beam energy is precisely known, the mass can be rescaled by using the beam energy

$$
M_{W}=M_{\text {meas }} \frac{E_{\text {beam }}}{E_{\text {meas }}}
$$

Monte Carlo study shows that both the absolute mass and the width are greatly improved as shown in Figure 2.42 . The expected precision is about $100 \mathrm{MeV}$, provided the rescaling is carefully studied and calibrated by using reliable Monte Carlo simulation of the detector.

In the leptonic decay, the direction of the neutrino is calculated from $\vec{p}_{\nu}=\vec{p}_{m i s s}=$ $-\left(\vec{p}_{h a d}+\vec{p}_{l}\right)$. The energy of the neutrino can be calculated in two ways. One of these methods makes use of the beam constraint such that $E_{\text {had }}=E_{\text {beam }}$. The other assumes $E_{\nu}=E_{b}-E_{l}$. The final energy-momentum of the neutrino is arrived at 


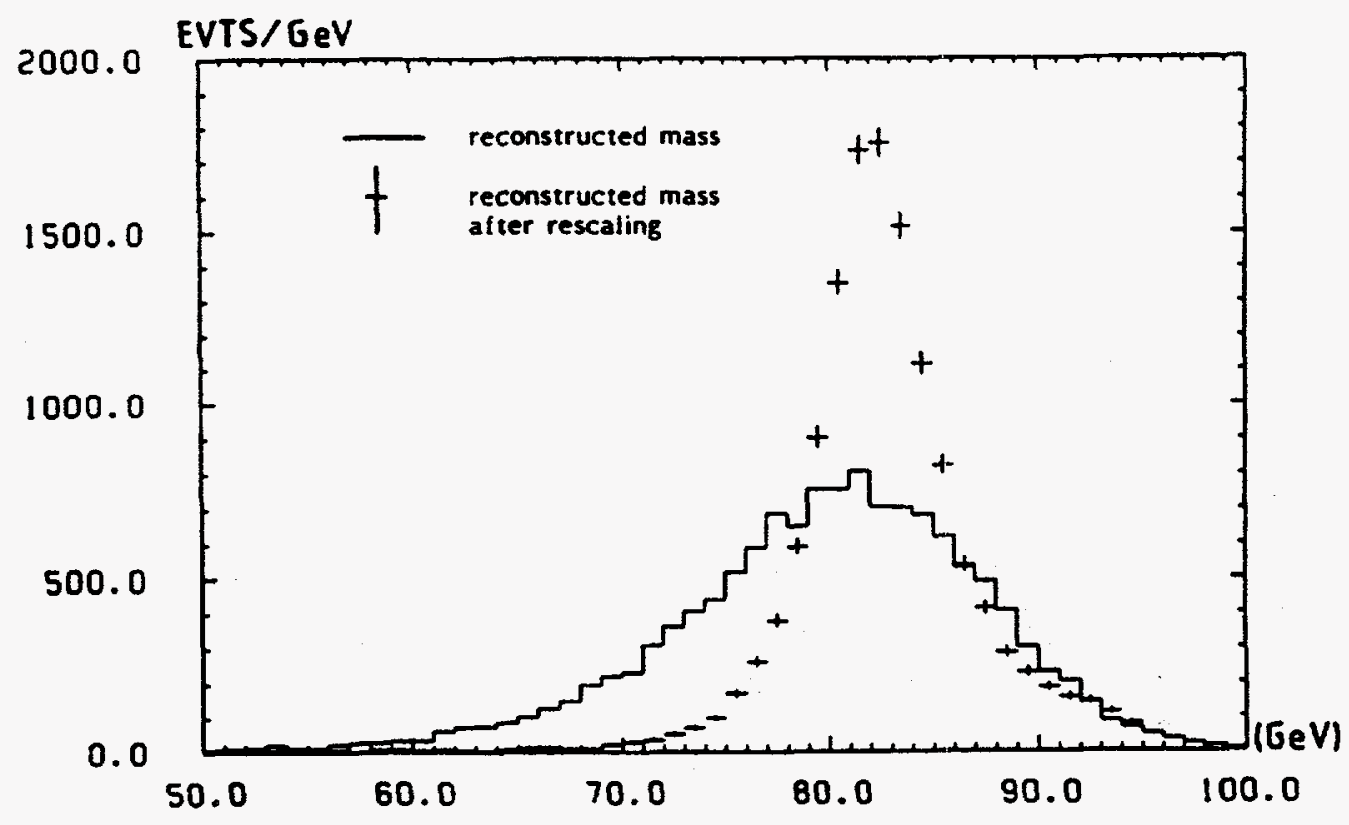

Figure 2.42: W Line Shape from Monte Carlo

after rescaling using Monte Carlo calibration. With an integrated luminosity of 500 $\mathrm{pb}^{-1}$ yielding statistics of about 1200 events, we expect a precision of about 100 $\mathrm{MeV}$ can be achieved.

(c) The $\mathrm{W}$ mass can be reconstructed from the end point of the leptonic energy spectrum. The lepton energy spectrum from the two body decay $W \rightarrow l \nu$ is directly related to the $\mathrm{W}$ mass. The kinematic limits are given by the Lorentz boost such that $W_{-}<E_{l}<W_{+}$, where $W_{ \pm}=E_{W}(1+\beta) / 2$. The energy spectrum from a Monte Carlo study using typical detector resolution is shown in Figure 2.43. We expect to measure the $\mathrm{W}$ mass to a precision of about $300 \mathrm{MeV}$. The precision using this method is inferior to the other methods because of several reasons: the width of $W$, the resolution from a single measurement, the lack of constraints, and the uncertainty in the absolute energy calibration of the detector.

We can expect that the mass of the $\mathrm{W}$ can be measured to within $100 \mathrm{MeV}$ after about one year of operation of LEP II above the W-pair production threshold. The ultimate precision is about $50 \mathrm{MeV}$ after three years of operation.

\section{The Search for the Higgs Boson}

While the Standard Model has been very successful in describing experimental results at energies available in laboratories today, there is as yet no experimental information on the mechanism for electroweak symmetry breaking which gives masses to particles. In the Standard Model, the simplest system of Higgs fields consists of a doublet and its conjugate. Three of the four real fields in this complex doublet are "eaten up" by the $W^{ \pm}$and $Z^{\circ}$, and only one real neutral Higgs boson $H^{\circ}$ remains to 


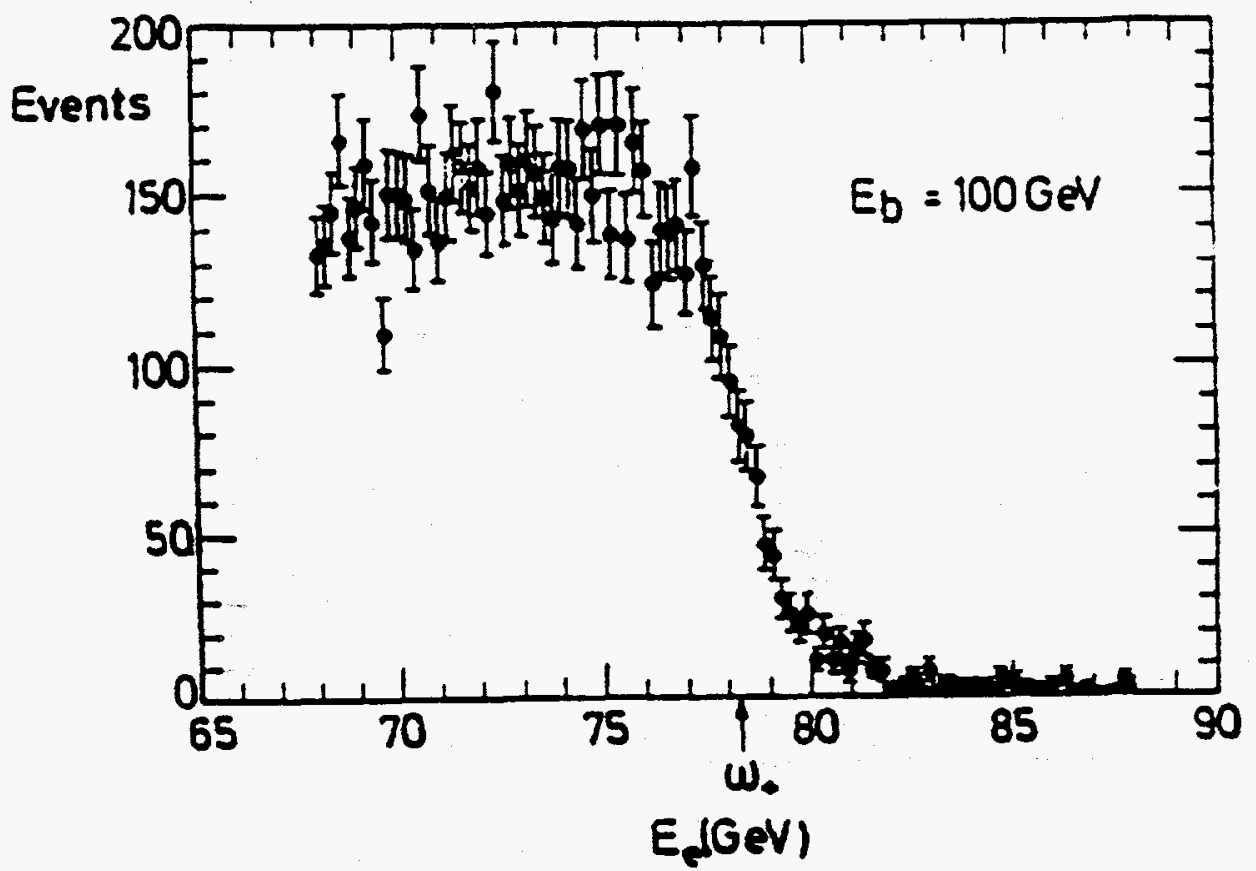

Figure 2.43: End Point of Energy Spectrum

be observed. There is however no theoretical prediction of the mass of $H^{0}$. OPAL has set a mass limit of $34 \mathrm{GeV}$ on $H^{0}$ using a partial sample of data obtained so far. The limit is approaching $42 \mathrm{GeV}$ if all LEP experiments are considered.

The minimal supersymmetric Standard Model contains two complex Higgs doublets for a total of eight real fields [46]. In addition to $W^{ \pm}$and $Z^{0}$, there are five Higgs bosons which may be experimentally observable. Two of these are neutral scalars $H_{1}^{0}$ and $H_{2}^{0}$, one is a neutral pseudoscalar $H_{3}^{0}$, and two are charged particles $H^{ \pm}$. Within the minimal supersymmetric Standard Model, the masses of these Higgs particles are related:

$$
\begin{aligned}
m\left(H_{1}^{0}\right) & <m_{Z} ; \quad m\left(H_{2}^{0}\right)>m_{Z} ; \\
m^{2}\left(H_{3}^{0}\right) & =m^{2}\left(H_{1}^{0}\right)+m^{2}\left(H_{2}^{0}\right)-m_{Z}^{2} ; \\
m^{2}\left(H^{ \pm}\right) & =m^{2}\left(H_{3}^{0}\right)+m_{W}^{2} .
\end{aligned}
$$

So one of the neutral scalar Higgs bosons is lighter than the Z; the pseudoscalar may or may not be lighter than the Z; and the charged Higgses are heavier than the W.

Therefore, the experimental search for the Higgs bosons is very important at LEP II, because one will be able to determine the existence of one (or more) Higgs bosons up to the mass of the $Z$. If no evidence is observed for a neutral Higgs boson, it will rule out the minimal supersymmetric model. On the other hand, if one neutral Higgs boson is observed below the mass of the $\mathrm{Z}$, it will be difficult to distinguish between the Standard Model, the minimal sypersymmetric Standard Model, and other extensions of these models.

The dominant process for the Standard Model neutral Higgs boson production 


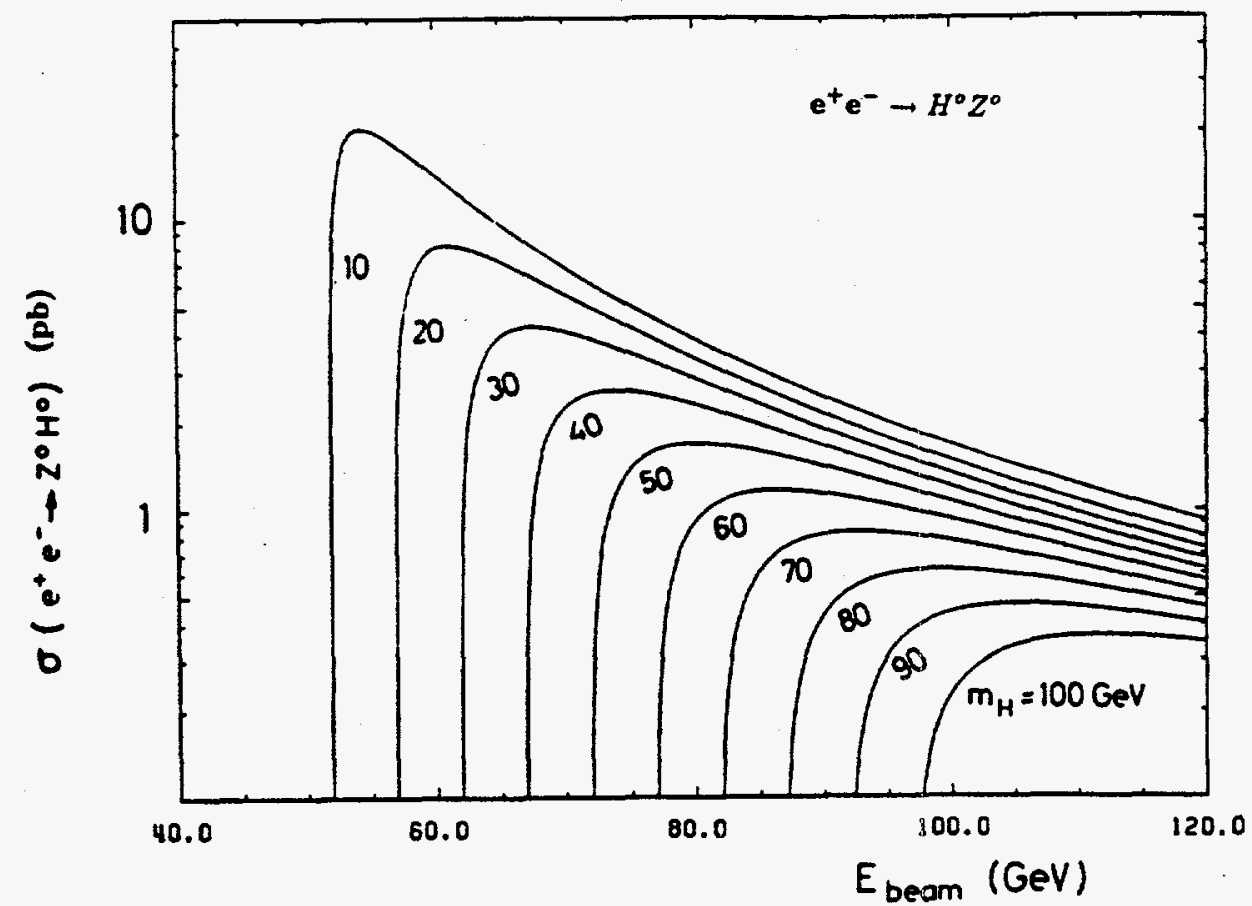

Figure 2.44: Cross sections for various Higgs masses

at LEP II energies is [47]

$$
e^{+} e^{-} \rightarrow Z^{*} \rightarrow H^{0} Z^{0}
$$

The cross sections for this process for various masses of $H^{0}$ are shown as a function of the beam energy in Figure 2.44. They range from $1 \mathrm{pb}$ to $0.2 \mathrm{pb}$ for $H^{0}$ mass between 40 and $100 \mathrm{GeV}$ at a beam energy of $100 \mathrm{GeV}$. Backgrounds to the Higgs signal come from $e^{+} e^{-} \rightarrow \gamma Z^{0}, W^{+} W^{-}$, and $Z^{0} Z^{0}$.

Operating at a c.m. energy of $200 \mathrm{GeV}$ with an average luminosity of $10^{31} \mathrm{~cm}^{-2} \mathrm{sec}^{-1}$, we can expect an integrated luminosity of $500 \mathrm{pb}^{-1}$ in two years at LEP II. If we tag the events with $Z^{0} \rightarrow l^{+} l^{-}$, we expect about 35 events for $50 \mathrm{GeV}$ Higgs and 20 events for $80 \mathrm{GeV}$ Higgs. Figures 2.45 show the jet-jet mass distributions recoiling against the $Z^{0}$ for $50 \mathrm{GeV}$ and $80 \mathrm{GeV}$ Higgs bosons respectively. As can be seen, it is relatively straightforward to identify the Higgs signal up to $80 \mathrm{GeV}$ in mass with appropriate cuts.

To search for the neutral Higgs of masses up to the beam energy is much more difficult. The process $e^{+} e^{-} \rightarrow Z^{0} Z^{0}$ has a cross section several times that of the signal. The production angular distribution is known and different from that of the production of the Higgs boson. It is possible to enhance the signal to background ratio by selecting events in the central angular region. Since the Higgs is expected to decay almost entirely into heavy quark pairs, we can determine the existence of the Higgs boson by comparing event rates in $b \bar{b}$ final states and in $l^{+} l^{-}$final states. This method clearly would require much more data.

In summary, LEP II will provide unique opportunities for the study of $W^{+} W^{-}$ production. In particular, we will be able to determine the triple gauge boson coupling and test the Standard Model. The precision measurement of the W mass 


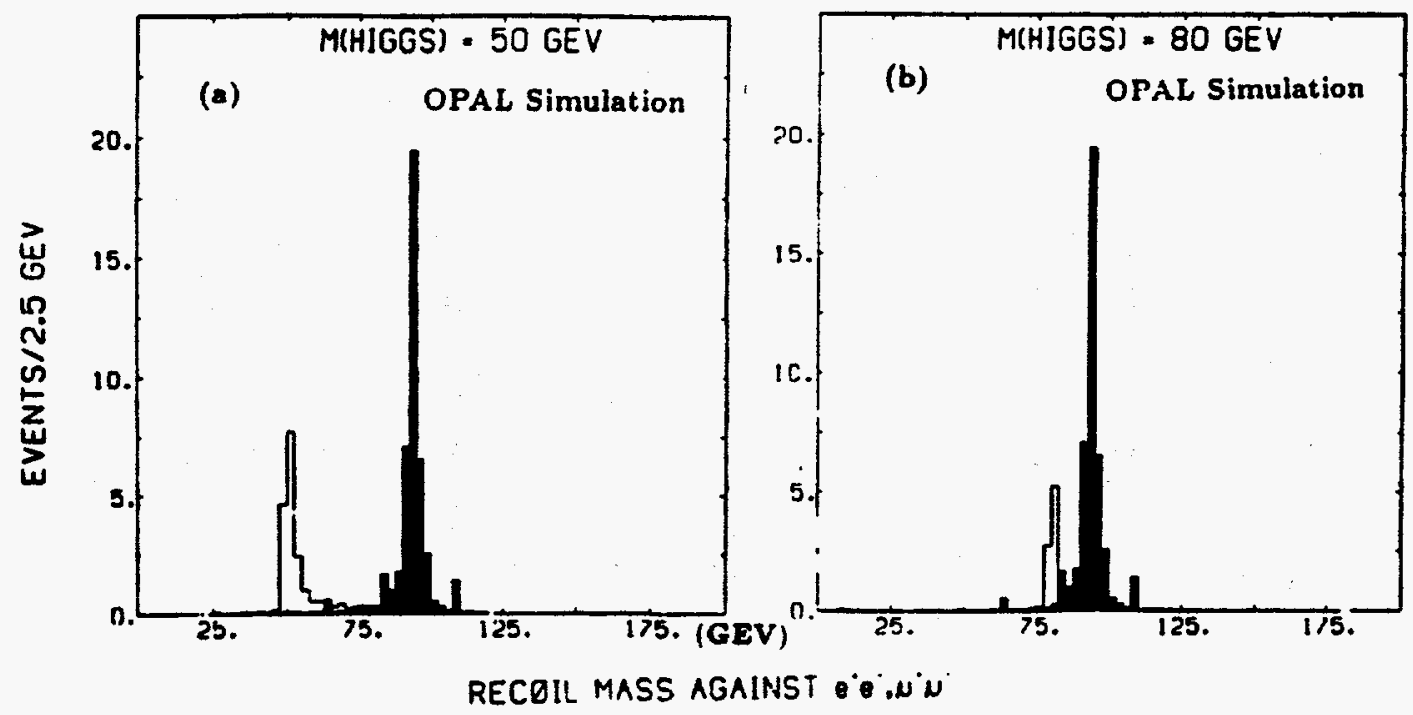

Figure 2.45: Jet-jet Mass Distributions recoiling against the Z: a) $m_{H}$ of $50 \mathrm{GeV}$ and b) $m_{H}$ of $80 \mathrm{GeV}$

will yield definitive information on the mass scale of new physics. The search for the Higgs boson will determine the validity of the Minimal Supersymmetric Standard Model. 


\subsubsection{Personnel}

The OPAL experiment at LEP is the major project of our group, and our responsibilities include operation of the Hadron Calorimeter strip system, the VAX 8700 computer in the OPAL data acquisition system, maintenance and development of online data acquisition and offline reconstruction software, and physics analysis.

The physicists participating in OPAL are M. Dittmar, W. Gorn, J. Layter, K. Riles, B. Shen, and G. VanDalen. Seven graduate students have been working on various aspects of the experiment. Three of them will complete their Ph.D. research within one year, and two others are expected to complete their work in two years. In addition, we have one visiting scientist, Y. Yang, from the People's Republic of China. 


\subsection{Neutrino Physics at LAMPF}

Neutrino interactions have been used throughout the development of particle physics to study a wide range of fundamental questions including the nature of the electroweak interaction, lepton number conservation, and as a probe of cosmology. Low energy neutrinos, such as those available at LAMPF, offer significant advantages in testing the fundamental properties of the neutrinos themselves. In the decade of the 80's as series of experiments at LAMPF have measured neutrino interactions at low energies. Since 1982 several members of our group, notably William Gorn and Gordon VanDalen, have participated in an experiment at LAMPF which established significant limits in the search for neutrino oscillations. [48]

We are now collaborating in a new program which is a natural successor to the previous experiment, and serves as a logical next step in the entire LAMPF neutrino program. The primary goal of the experiment is to search for neutrino oscillations to the levels of $10^{-2} \mathrm{eV}^{2}$ in mass difference and $2 \times 10^{-4}$ in mixing. The greatly extends the range explored by accelerator based searches.

The new experiment, the Large Scintillation Neutrino Detector, or LSND [49], will consist of 200 tons of dilute mineral oil-liquid scintillator located near the LAMPF beam stop neutrino source. The primary focus of LSND is neutrino oscillations, although a range of related neutrino interactions will also be investigated. The collaboration of 33 physicists from LAMPF and 5 universities has prepared a proposal which has been approved with the highest ranking by the Los Alamos PAC. The first stages of construction are taking place in 1990-91, and data acquisition with the complete detector will begin in summer of 1992 .

\subsubsection{The Detector}

The detector is similar to a large water Cerenkov device but with better angular, position, and energy resolution due to more light collected from scintillation, and the improvements in Cerenkov imaging from higher index of refraction, longer radiation length, and lower density of mineral oil compared to water. The expected energy, position and angular resolutions for a $45 \mathrm{MeV}$ electron are $<5 \%,<25 \mathrm{~cm}$, and $<15^{\circ}$ respectively. Protons are identified by the absence of a Cerenkov cone, and neutrons will be tagged by the $2.2 \mathrm{MeV}$ photon from neutron absorption on a free proton. The superb event timing and vertex reconstruction allows us to separate neutrino induced events from pion decay in flight, decay at rest, and cosmic ray backgrounds using the $200 \mathrm{MHz}$ fine structure in the LAMPF beam.

The LSND detector is based on an extension of a technique that has been used in large water Cherenkov devices such as Kamioka and IMB. These detectors are capable of reconstructing the energy and direction of low-energy electrons through the Cherenkov light emitted in the liquid. Kamioka, in particular, has observed recoil electrons from solar neutrinos below $10 \mathrm{MeV}$. The technique of imaging-Cherenkov 


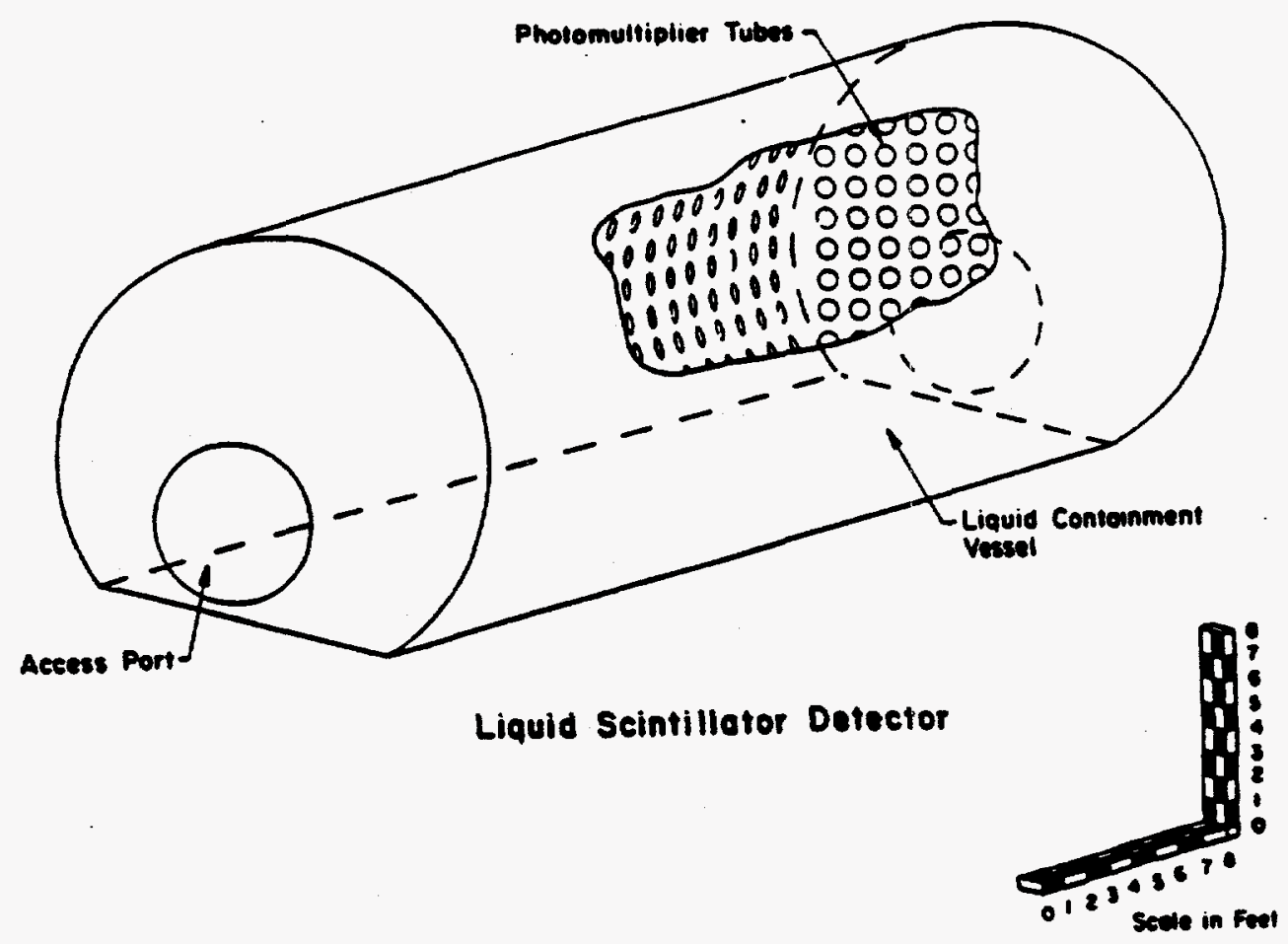

Figure 2.46: Schematic view of the LSND detector

detectors for observing low-energy, simple-topology events is well established. Instead of using water as the target medium, it is proposed to use mineral oil with a refractive index of 1.47. This medium is composed approximately of $\mathrm{CH}_{2}$ which is superior for detection of low-energy electrons by virtue of its lower density, higher refractive index, and lower radiation length. The addition of small quantities of scintillator to the mineral oil gives the additional benefit that the energy resolution is substantially improved and offers the possibility of detecting muons below Cherenkov threshold and low-energy neutrons - a crucial element in lowering the background to one of the oscillation signals in the detector. The performance that is expected in this experiment is dependent on the use of large-area, precise-timing phototubes that have been developed recently. All of these improvements in the imaging technique make it feasible to search for neutrino oscillations with high sensitivity at LAMPF.

The proposed detector is shown in Figure 2.46. It consists of a cylindrical tank of dilute mineral-oil-based liquid scintillator approximately $6 \mathrm{~m}$ in diameter by 9 $m$ long with an active mass of 200 tons. The tank will reside inside the existing E645 veto shield, which is located $27 \mathrm{~m}$ downstream of the proton beam stop and is at an angle of approximately $17^{\circ}$ to the beam direction. The tank will be made of $1 \mathrm{~cm}$ thick steel and have $85010^{n}$ diameter, very low time jitter $(\sigma \sim 1 \mathrm{~ns})$ photomultiplier tubes mounted uniformly over the inside surface.

The 850 10" photomultiplier tubes cover $28 \%$ of the surface area of the tank with sensitive photocathode. These tubes, shown in Figure 2.47, are manufactured by Burle Corporation (C83061E photomultiplier tube) and have excellent timing resolution, $2.3 \mathrm{~ns}$ FWHM for single photons and full-face illumination. They also have 


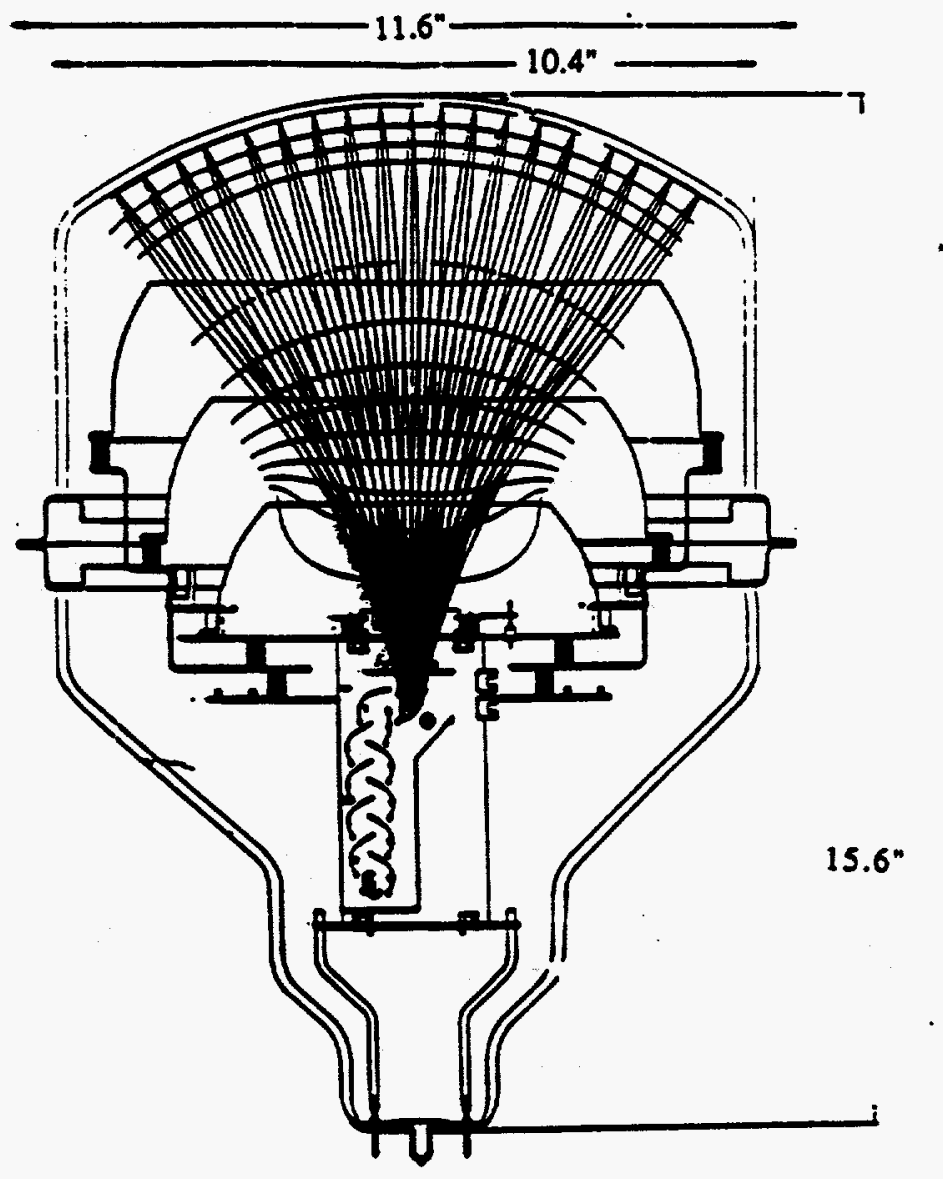

Figure 2.47: Section through the Burle C83061E photomultiplier tube 


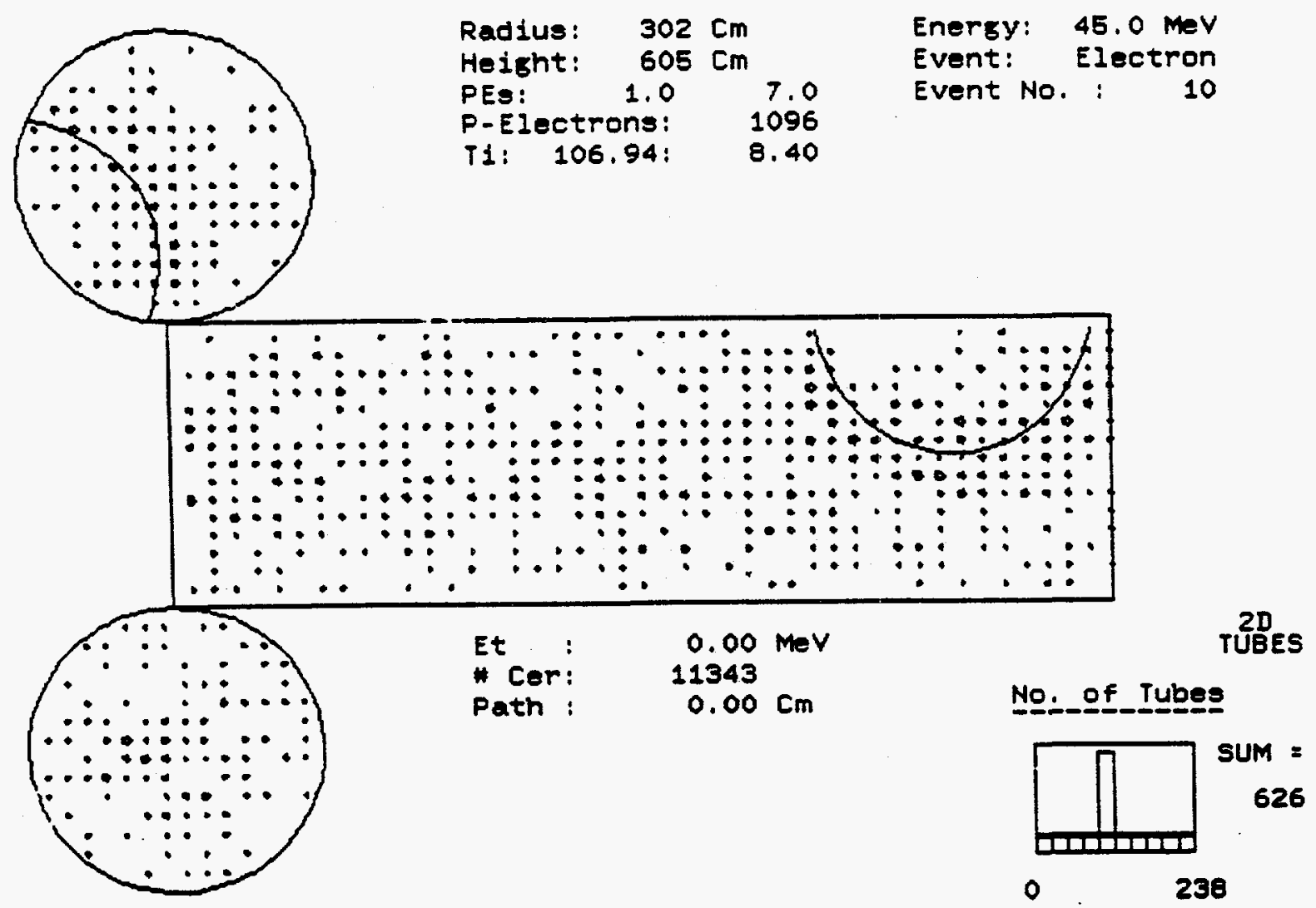

Figure 2.48: Monte Carlo event seen in the detector.

single photoelectron separation and typical noise rates $<10 \mathrm{kHz}$. Pre-production phototubes are being tested and evaluated within the collaboration.

We plan to use a dilute concentration of scintillator such that about $20 \%$ of the total light output will be Cherenkov light and $80 \%$ scintillation light. This concentration may vary after we perform further tests and optimizations. Mineral oil has advantages relative to water for the detection of Cherenkov light as it has a higher index of refraction, a lower density so that electrons travel farther before stopping, and a sharper Cherenkov ring due to the longer radiation length. The absorption and emission spectra of the scintillator

Figure 2.48 shows a typical $45 \mathrm{MeV}$ electron event generated by the Monte Carlo. Each number corresponds to a hit photomultiplier tube and equals the number of photoelectrons. The detector cylinder has been unrolled to clearly show the phototube hit pattern. Note that the location of the Cherenkov ring can be seen by eye.

The front-end electronics and data acquisition system to handle the event triggers and above rates is being designed to record and selectively process the pulse histories 
Table 2.5: Event rates per 130 days for neutrinos from pion decay at rest.

Process Cross Section $\left(\mathrm{cm}^{2}\right)$ Acceptance Events Comments

$\begin{array}{ccccc}\bar{\nu}_{e} \rightarrow e^{+} n & 1.3 \times 10^{-40} & 0.234 & 19,350 & \text { max.-mixing, } \\ & & & & E_{e}>37 \mathrm{MeV} \\ \nu_{e}{ }^{12} C \rightarrow e^{12} N & 1.46 \times 10^{-41} & 0.342 & 1588 & E_{e}>10 \mathrm{MeV} \\ \nu_{e}{ }^{13} C \rightarrow e^{13} N & 1.09 \times 10^{-40} & 0.490 & 190 & E_{e}>10 \mathrm{MeV} \\ \nu e^{-} \rightarrow \nu e^{-} & 4.0 \times 10^{-43} & 0.331 & 340 & E_{e}>10 \mathrm{MeV} \\ \nu C \rightarrow \nu C^{*} & 7.5 \times 10^{-42} & 0.509 & 1215 & C^{*} \text { emits } \\ & & & & 15.11 \mathrm{MeV} \gamma\end{array}$

Table 2.6: Event rates per 130 days for neutrinos from decay in flight Process Cross Section $\left(\mathrm{cm}^{2}\right)$ Acceptance Events Comments

$\begin{array}{ccccl}\pi^{0} \rightarrow \nu \bar{\nu} & & 0.509 & \approx 10^{8} & \text { for B.R. }=1 \\ \eta \rightarrow \nu \bar{\nu} & & 0.509 & \approx 10^{4} & \text { for B.R. }=1 \\ \nu_{\mu} p \rightarrow \nu_{\mu} p & 4.5 \times 10^{-40} & 0.234 & 1970 & E_{p}>20 \mathrm{MeV} \\ \nu_{e} C \rightarrow e^{-} N & 3 \times 10^{-39} & 0.509 & 14,310 & \text { max.-mixing, } \\ & & & & E_{e}>60 \mathrm{MeV} \\ \nu_{\mu} C \rightarrow \mu^{-} N & 6 \times 10^{-40} & 0.283 & 910 & E_{\mu}>10 \mathrm{MeV} \\ \bar{\nu}_{\mu} p \rightarrow \mu^{+} n & 5 \times 10^{-40} & 0.283 & 300 & E_{\mu}>10 \mathrm{MeV} \\ \bar{\nu}_{\mu} C \rightarrow \mu^{+} B & 1.5 \times 10^{-40} & 0.283 & 45 & E_{\mu}>10 \mathrm{MeV} \\ \nu_{\mu} C \rightarrow \nu_{\mu} C^{*} & 3.5 \times 10^{-41} & 0.509 & 170 & C^{*} \text { emits } \\ & & & & 15.11 \mathrm{MeV} \gamma \\ \nu_{\mu} e^{-} \rightarrow \nu_{\mu} e^{-} & 2.5 \times 10^{-43} & 0.509 & 9 & E_{e}>10 \mathrm{MeV}\end{array}$

(charge and time) of each of the 850 photomultiplier tubes with minimal dead time and very high system reliability.

\subsubsection{Event Rates}

An estimate of LSND event rates is shown in Table 2.5 for events from pion decays at rest, and in Table 2.6 for neutrinos from pion decay in flight. All rates are per 130 days of running (one "LAMPF year"). 


\subsubsection{The Physics}

$\nu_{\mu} \rightarrow \nu_{e}$ Oscillations

A high-sensitivity search for $\nu_{\mu} \rightarrow \nu_{e}$ oscillations can be performed by using neutrinos with energies greater than $\sim 80 \mathrm{MeV}$ from pion decay in flight in the beam dump. If oscillations occur, then $\nu_{\mu}$ neutrinos oscillate into $\nu_{e}$ neutrinos, which would be detected via the reaction $\nu_{e} C \rightarrow e^{-} N$. The event signature for these oscillations is a single high-energy electron with energy in the approximate range $60<E_{e}<180 \mathrm{MeV}$. The lower-energy events in this range will be most useful for establishing an oscillation limit at low $\Delta m^{2}$, while the higher-energy events will be most important at high $\Delta m^{2}$.

Although only about $2.5 \%$ of the pions decay in flight before stopping in the A6 beam stop, the cross section for $\nu_{\mathrm{e}} C \rightarrow e^{-} N$ rises rapidly with neutrino energy. A simulation of the A6 beam line has determined the $\pi^{+}$decay-in-flight neutrino flux at a distance of $\sim 27 \mathrm{~m}$ from the beam stop to be $2.0 \times 10^{12} \nu_{\mu} / \mathrm{cm}^{2}\left(3.9 \times 10^{11} \bar{\nu}_{\mu} / \mathrm{cm}^{2}\right.$ from $\pi^{-}$decay in flight) for $E_{\nu}>80 \mathrm{MeV}$ after 130 days of running at LAMPF ( $\sim 3000$ actual hours) or 8678 Coulombs. Using $5.9 \times 10^{30} \mathrm{C}$ nuclei, a $\nu_{e} C \rightarrow e^{-} N$ cross section of $2.8 \times 10^{-39} \mathrm{~cm}^{2}$, and an acceptance of 0.513 , we estimate the number of $\nu_{e} C \rightarrow e^{-N}$ events per 130 days for maximal neutrino mixing to be

$$
\left(2.0 \times 10^{12}\right)\left(2.8 \times 10^{-39}\right)(0.513)\left(5.9 \times 10^{30}\right)=16,950 .
$$

The $\nu_{e} C \rightarrow e^{-N}$ acceptance (0.513) is the product of the shield live time (86.0\%), the $25 \mathrm{~cm}$ fiducial volume cut $(66.3 \%)$, and the electron identification efficiency (90\%), which results from rejecting possible proton events. The shield live time is the total live time and includes dead-time losses from the hardware trigger and the off-line analysis.

Our per-event sensitivity after two years of data collection is $2.9 \times 10^{-5}$, which is based on a total of 33,900 $\nu_{e} C \rightarrow e^{-N}$ events for maximal mixing. Using a background level of $3.8 \times 10^{-4}$, or 13 events, a $90 \%$ confidence level limit of $2.7 \times 10^{-4}$ for $\nu_{\mu} \rightarrow \nu_{e}$ oscillations at $\Delta m^{2}>3 \mathrm{eV}^{2}$ can then be obtained. Note that the cosmic-ray background level can be precisely determined from the beam-off data, while the $\mu^{+}$decay-in-flight and $\pi^{+} \rightarrow e^{+} \nu_{e}$ background can be determined in two ways: from the Monte Carlo simulation and from fitting to the energy distribution, as the background energy distribution is different from the energy distribution of the oscillation signal. Figure 2.49 shows the limiting oscillation curve in the $\Delta m^{2}$ vs $\sin ^{2} 2 \theta$ parameter space, which is seen to be comparable to the $\bar{\nu}_{\mu} \rightarrow \bar{\nu}_{e}$ limiting oscillation curve discussed in the next section.

$\bar{\nu}_{\mu} \rightarrow \bar{\nu}_{e}$ Oscillations

A high-sensitivity search for $\bar{\nu}_{\mu} \rightarrow \bar{\nu}_{e}$ oscillations can be performed with neutrinos from muon decay at rest with energies greater than $\sim 40 \mathrm{MeV}$. If oscillations occur, 


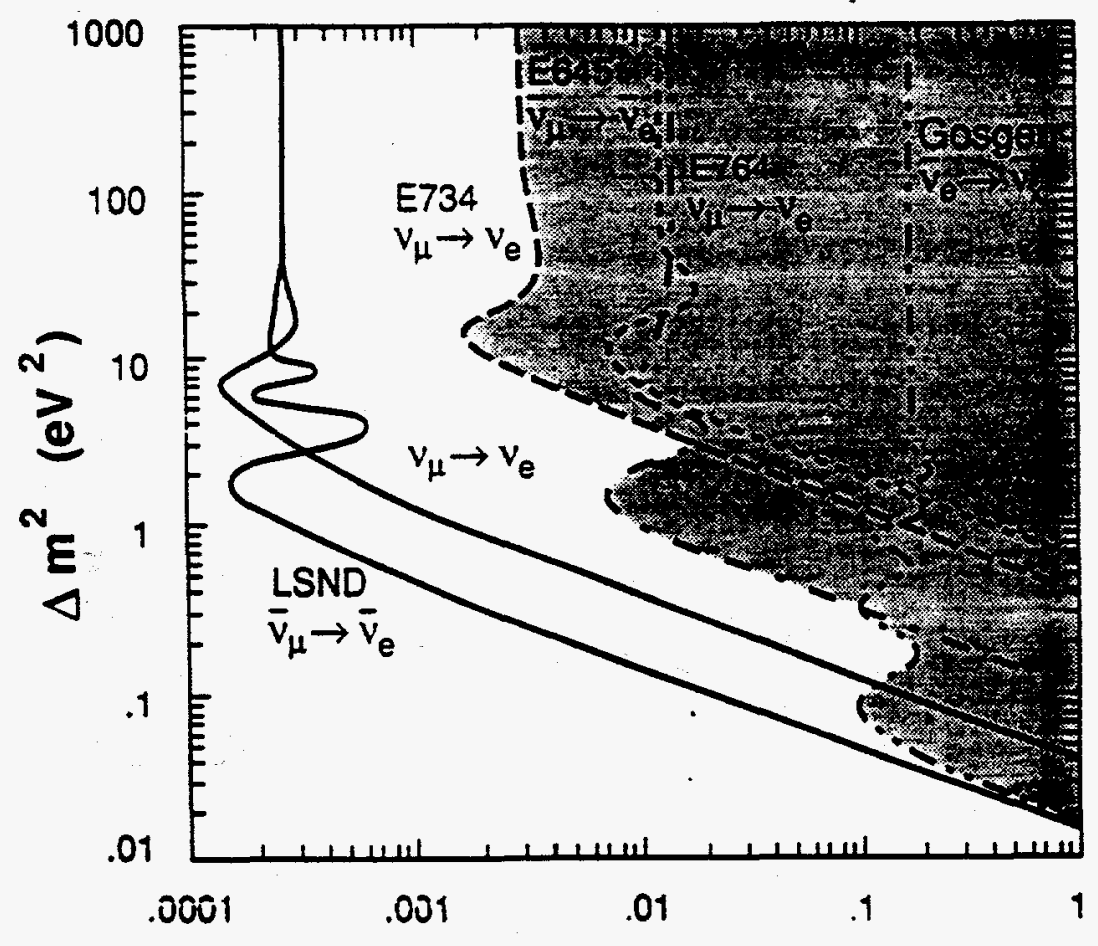

Figure 2.49: Limiting oscillation curve in the $\Delta m^{2}$ vs $\sin ^{2} 2 \theta$ parameter space for accelerator experiments.

then $\bar{\nu}_{\mu}$ neutrinos oscillate into $\bar{\nu}_{e}$ neutrinos, which are detected via the chargedcurrent reaction on a free proton, $\bar{\nu}_{e} p \rightarrow e^{+} n$, followed by the capture of the recoil neutron on hydrogen. The event signature is an electron in the energy range $37<$ $E_{e}<50 \mathrm{MeV}$ in coincidence with a $2.2 \mathrm{MeV} \gamma$ from neutron absorption on a free proton within $1 \mathrm{~m}$ and $0.5 \mathrm{~ms}$ of the electron event. These position and time requirements may vary somewhat after data are obtained and a further optimization is performed.

A simulation of the $\mathrm{A} 6$ beam line has determined the decay-at-rest neutrino flux at a distance of $\sim 27 \mathrm{~m}$ from the beam stop to be $5.4 \times 10^{13} \nu_{\mu} / \mathrm{cm}^{2}$ from pion decay at rest and an equal number of $\nu_{\mathrm{e}}$ and $\bar{\nu}_{\mu}$ from muon decay at rest, after 130 days of running at LAMPF ( 3000 actual hours) or 8678 Coulombs. Using $11.8 \times 10^{30}$ free protons, a $\bar{\nu}_{e} p \rightarrow e^{+} n$ cross section of $1.5 \times 10^{-40} \mathrm{~cm}^{2}$, and an acceptance of 0.173 , we can estimate the number of $\bar{\nu}_{\mathrm{e}} p \rightarrow e^{+} n$ events per year for maximal neutrino mixing to be

$$
\left(5.4 \times 10^{13}\right)\left(1.5 \times 10^{-40}\right)(0.173)\left(11.8 \times 10^{30}\right)=16,540 .
$$

The $0.173 \bar{\nu}_{e} p \rightarrow e^{+} n$ acceptance is the product of the shield live time (74.3\%), the $25 \mathrm{~cm}$ fiducial volume cut $(66.3 \%)$, the electron identification efficiency $(90 \%)$, the fraction of events with $E_{\mathrm{e}}>37 \mathrm{MeV}(45.9 \%)$, and the recoil neutron detection efficiency (85.0\%). The shield live time is the total live time and includes dead-time losses from the hardware trigger and the off-line analysis. 


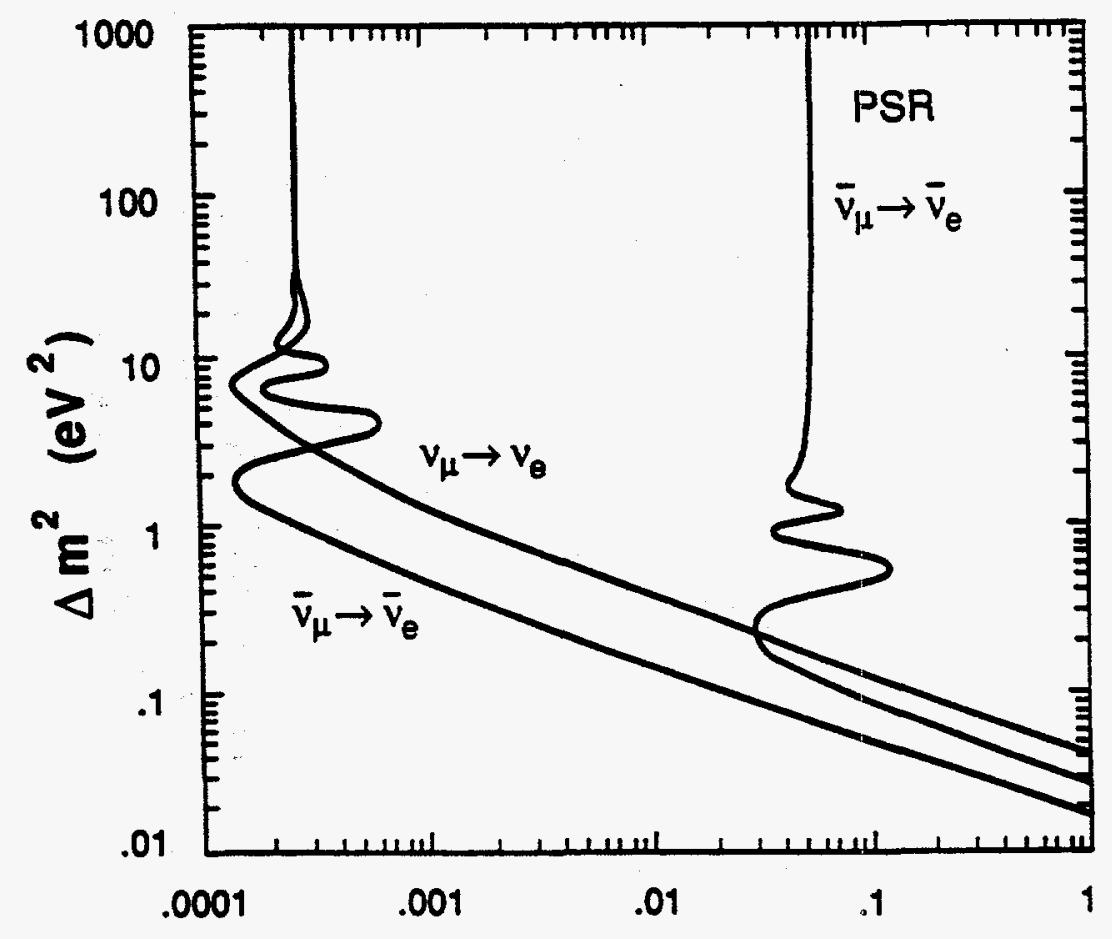

Figure 2.50: Limiting oscillation curve in $\Delta m^{2}$ vs $\sin ^{2} 2 \theta$ space for LSND

Our per-event sensitivity after two years of data collection is $3.0 \times 10^{-5}$, which is based on a total of $33,080 \bar{\nu}_{e} p \rightarrow e^{+} n$ events for maximal mixing. Using a background level of $3.9 \times 10^{-4}$, or 12.8 events in two years, a $90 \%$ confidence level limit of $2.7 \times 10^{-4}$ for all $\bar{\nu}_{\mu} \rightarrow \bar{\nu}_{e}$ oscillations with $\Delta m^{2}>1 \mathrm{eV}^{2}$ can be obtained. For $\sin ^{2} 2 \theta=1$, the $90 \%$ confidence level limit on $\Delta m^{2}$ is $1.7 \times 10^{-2} \mathrm{eV}^{2}$.

Note that the sensitivity estimates are reasonable because the cosmic-ray background level can be precisely determined from the beam-off data, while the $\mu^{-}$ decay-at-rest background can be determined from fitting to the combined energy distribution. Figure 2.50 shows the limiting oscillation curve in the $\Delta m^{2}$ vs $\sin ^{2} 2 \theta$ parameter space.

\section{$\bar{\nu}_{\mu} \rightarrow \bar{\nu}_{e}$ Oscillations with PSR Neutrinos}

A search for $\bar{\nu}_{\mu} \rightarrow \bar{\nu}_{e}$ oscillations at low $\Delta m^{2}$ can also be performed with neutrinos from the Proton Storage Ring (PSR). The event signature, an electron from the reaction $\bar{\nu}_{e} p \rightarrow e^{+} n$ followed by the capture of the recoil neutron on hydrogen, is the same as discussed in the previous section, except that there is the additional constraint that the event time must correspond to within a few muon lifetimes with one of the short (270 ns) PSR spills. There is essentially no cosmic-ray, beam-neutron, or beam-neutrino background because the duty factor is so low $\left(\sim 10^{-4}\right)$. This measurement will be done with the detector in the same location and concurrently with the measurements described above. 
The event rate can be estimated by comparison with the last sections. As the PSR intensity is $\sim 100 \mu \mathrm{A}$, the neutrino flux at a distance of $\sim 209 \mathrm{~m}$ from the PSR beam stop is estimated to be $1.13 \times 10^{11} \nu_{\mu} / \mathrm{cm}^{2}$ from pion decay at rest and an equal number of $\nu_{e}$ and $\bar{\nu}_{\mu}$ from muon decay at rest after 130 days of running at LAMPF ( 3000 actual hours) or 8678 Coulombs. Using $11.8 \times 10^{30}$ free protons, a $\bar{\nu}_{\mathrm{e}} p \rightarrow e^{+} n$ cross section ${ }^{13}$ of $1.5 \times 10^{-40} \mathrm{~cm}^{2}$, and an acceptance of 0.233 we can estimate the number of $\bar{\nu}_{e} p \rightarrow e^{+} n$ events per year for maximal neutrino mixing to be

$$
\left(1.13 \times 10^{11}\right)\left(1.5 \times 10^{-40}\right)(0.233)\left(11.8 \times 10^{30}\right)=46 .
$$

The $0.233 \bar{\nu}_{e} p \rightarrow e^{+} n$ acceptance is the product of the the $25 \mathrm{~cm}$ fiducial volume cut $(66.3 \%)$, the electron identification efficiency $(90 \%)$, the fraction of events with $E_{e}>37 \mathrm{MeV}(45.9 \%)$, and the recoil neutron detection efficiency (85.0\%). The acceptance does not include the 0.743 veto shield efficiency due to the low PSR duty factor.

Our per-event sensitivity after two years of data collection is $1.1 \times 10^{-2}$, which is based on a total of $92 \bar{\nu}_{e} p \rightarrow e^{+} n$ events for maximal mixing. As there is essentially no background, a $90 \%$ confidence level limit of $2.5 \times 10^{-2}$ for $\bar{\nu}_{\mu} \rightarrow \bar{\nu}_{e}$ oscillations can be obtained at the peak sensitivity of $\Delta m^{2}=0.2 \mathrm{eV}^{2}$. For $\sin ^{2} 2 \theta=1$, the $90 \%$ confidence level limit on $\Delta m^{2}$ is $3 \times 10^{-2} \mathrm{eV}^{2}$. Figure 2.50 also shows the oscillation limit in the $\Delta m^{2}$ vs $\sin ^{2} 2 \theta$ parameter space. This $\bar{\nu}_{\mu} \rightarrow \bar{\nu}_{e}$ oscillation limit would be comparable to the limit obtained in the previous section for low values of $\Delta m^{2}$.

\section{Neutrino Oscillation Summary}

LSND does two largely independent neutrino oscillation experiments with similar sensitivities that can be performed concurrently. Simply stated, one experiment looks for high-energy $\nu_{e}\left(80<E_{\nu}<200 \mathrm{MeV}\right)$ produced by conversion to $\nu_{e}$ of the $\nu_{\mu}$ from the decay-in-flight component of the beam-dump neutrino beam. Simultaneously, another experiment looks for low-energy $\bar{\nu}_{e}$ produced by conversion of $\bar{\nu}_{\mu}\left(40<E_{\nu}<53 \mathrm{MeV}\right)$ from the decay-at-rest component of the same beam. In each experiment the incident beam, the event signature, and the backgrounds are different. Consequently, with similar sensitivities as shown in Figure 2.50, the two experiments (plus the PSR experiment) provide important redundancy in addition to significantly wider coverage of the $\Delta m^{2}-\sin ^{2} 2 \theta$ space than all previous accelerator searches for $\nu_{\mu} \rightarrow \nu_{e}\left(\bar{\nu}_{\mu} \rightarrow \bar{\nu}_{e}\right)$ oscillations combined.

The experimental techniques involved in accomplishing these searches are not especially demanding. The use of dilute mineral-oil-based liquid scintillator to allow the energetic electrons (positrons) to be detected by Cherenkov radiation and the neutron capture $2.2 \mathrm{MeV}$ gamma ray (from $\bar{\nu}_{e} p \rightarrow e^{+} n$ ) to be identified by scintillation light is novel. It is worth pursuing in its own right for other possible applications and can be empirically validated in a reasonable scale prototype well before large-scale construction begins. 
From the experience gained by E645, several members of which are also members of the LSND collaboration, the backgrounds in the LAMPF neutrino area are well understood. It is unlikely that any appreciable surprise will come from that quarter.

It is possible that appreciable running time with the pulsed proton beam from the PSR may be acquired by LSND, again at the same time as the data-taking described above. This additional attack on the neutrino oscillation problem is less sensitive to low mixing strength at large $\Delta m^{2}$ than the high beam intensity experiments, but it has good $\Delta m^{2}$ sensitivity in the region of large mixing.

In short, neutrino oscillation searches proposed for LSND constitute a powerful program aimed at physics of topical interest and with every likelihood of successful achievement of that aim. Furthermore, the program makes use of and builds on the investment in and the experience gained from the previous neutrino oscillation experiments at LAMPF.

\section{Other Physics}

There are many other physics objectives that can be pursued with the liquid scintillator detector. Searching for $\bar{\nu}_{\mu} \rightarrow \bar{\nu}_{e}$ oscillations is equivalent to searching for the lepton number violating decay $\mu^{+} \rightarrow e^{+} \nu_{\mu} \bar{\nu}_{e}$. Limits on this decay are directly comparable to limits on neutrino oscillations, so that we should be sensitive to branching ratios as low as $10^{-4}$. A branching ratio limit this low would be very interesting theoretically, and would complement present searches for muonium-antimuonium conversion.

The $\nu C \rightarrow \nu C^{*}(15.11 \mathrm{MeV} \gamma)$ neutral-current reaction, one of the only neutrinonuclear neutral-current reactions that can be easily observed, will be measured to approximately $10 \%$ accuracy and will be recognized by the detection of the 15.11 $\mathrm{MeV} \gamma$ emitted by the excited carbon nucleus when it decays to the ground state. Our energy resolution at $15 \mathrm{MeV}$ is $<10 \%$, so that we should observe a peak in the 13.5-16.5 MeV range. A precision measurement of this process is especially important because it allows a calibration of the calculations used in current models of neutrino-nucleus interactions during supernova bursts. We are able to measure this reaction at two energies, at about $30 \mathrm{MeV}$ using decay-at-rest neutrinos and at about $150 \mathrm{MeV}$ using decay-in-flight neutrinos, and we are able to separate these two classes of events with the $200 \mathrm{MHz}$ LAMPF beam structure as the decay-in-flight events will be synchronous with the proton spill RF structure.

The rare decays $\pi^{\circ} \rightarrow \nu \bar{\nu}$ and $\eta \rightarrow \nu \bar{\nu}$, followed by $\nu_{e} C \rightarrow e^{-} N$, can be searched

to sensitivities of about $10^{-8}$ and $10^{-4}$, respectively, with very little background because the neutrinos from these decays are extremely energetic. These decays are forbidden for massless Weyl neutrinos and can proceed only if neutrino states of both chiralities exist or if lepton number is not conserved. The current best limits for these decays are $\Gamma\left(\pi^{\circ} \rightarrow \nu_{e} \bar{\nu}_{e}\right) / \Gamma\left(\pi^{\circ} \rightarrow\right.$ all $)<5.0 \times 10^{-7}$ and $\Gamma\left(\eta \rightarrow \nu_{e} \bar{\nu}_{e}\right) / \Gamma(\eta \rightarrow$ all $)<$ $1.0 \times 10^{-3}$ from the LAMPF E645 experiment. The expected branching ratios of 
these decays in the standard model for reasonable neutrino masses are much lower than our sensitivities; however, branching ratios as large as the current limit are possible in various phenomenological models.

We shall also measure the $\nu_{e} C$ and $\nu_{\mu} C$ charged-current scattering cross sections and the $\nu e$ elastic scattering cross section to approximately $10-15 \%$ accuracy. These measurements would test present theories of neutrino-nucleus scattering and provide an estimate of $\sin ^{2} \theta_{W}$, the fundamental parameter of the standard model of electroweak interactions. The electron angular distribution in the $\nu e$ elastic scattering process is strongly peaked in the neutrino direction, while it is slightly peaked in the backward direction for the charged-current reaction. The $\nu_{e} C \rightarrow e^{-N}$ chargedcurrent interaction is dominated by ${ }^{12} \mathrm{C}$, as the fraction of ${ }^{13} \mathrm{C}$ nuclei is only about $1.1 \%$ of the total. Nevertheless, we shall be able to measure the ${ }^{13} \mathrm{C}$ component separately due to the larger $\nu_{e}^{13} C \rightarrow e^{-N}$ cross section and the higher electron energy.

Finally, we will obtain a large sample of neutrino-proton elastic-scattering events, where the neutrinos are from pion decay in flight. The cross section for $\nu p \rightarrow \nu p$ is proportional to $G_{A}^{2}+F_{1}^{2}+\left(Q^{2} / 4 m_{p}^{2}\right) F_{2}^{2}$ at low $Q^{2}$. The term involving $G_{A}$ is dominant, and a measurement of the cross section at low $Q^{2}$ affords a method of measuring $G_{A}$ directly. The principal component of $G_{A}$ is isovector, which is known from neutron decay at low $Q^{2}$. Any additional contribution (an isoscalar term, for example) would affect the total cross section. We expect to make a preliminary search for recoil protons from neutrino-proton elastic scattering in the 20 to $60 \mathrm{MeV}$ range. These protons will be coherent with the beam spill from LAMPF in contrast to proton recoil from cosmic-ray or beam neutrons. A preliminary estimate of this background gives a signal to noise as evidenced by the $200 \mathrm{MHz} \mathrm{RF}$ time structure of about 1.

\subsubsection{Personnel}

Construction of the LSND begins in 1990-91 and continues in 1991-92. Data acquisition with the LAMPF beam should extend through 1992-1993 (setup and testing), and 1993-95 (physics data). UC Riverside is primarily responsible for the data acquisition computing hardware and software and is also involved in phototube testing and front-end readout design.

In 1990-91 a research assistant (William Strossman) will work on data acquisition and tube testing. A post-doc will be hired with university funds. In 1991-92 a second research assistant will be hired. W. Gorn and B. O'Neill will participate in the design of the data acquisition system. Since this system will be composed of modules from the existing OPAL data acquisition system, the design work requires a relatively small amount of time. G. VanDalen and B. Shen will devote approximately $15 \%$ of their research time to the LSND project. 


\subsection{Future Directions}

\subsubsection{Immediate future of OPAL physics}

OPAL hopes by the end of the 1990 run to have collected enough data during scanning to measure the total $\mathrm{Z}$ width to an accuracy of about $10 \mathrm{MeV}$, and the leptonic widths to about $1 \mathrm{MeV}$. The prospects of directly measuring the invisible decay width to neutrinos have been discussed earlier. The width measurements will allow $\sin ^{2} \theta_{W}$ to be determined to an accuracy of 0.003 . Additional information on $\sin ^{2} \theta_{W}$ comes from measuring the forward-backward asymmetry of lepton pairs and from measuring tau polarization.

Particle searches will continue. Although many possible particles have already been ruled out for masses below the beam energy, there remains the ongoing search for the Higgs particle, whose mass limits should continue to improve slowly with increasing integrated luminosity. There also remain particles whose production depends on unknown mixing angles, for example, singlet heavy neutral lepton production and excited lepton production. Our sensitivity to mixing angles should improve linearly with increasing luminosity.

As more data come in, the possibility of searching for rare decays becomes more promising. Four-fermion decays have already been seen. Of more interest, though difficult to establish, are triple-gluon decays. Triple-photon decays should be highly suppressed. Their observation would therefore be of great interest. There are also forbidden decays such as lepton-flavor-violating decay to electron and tau, or nearly forbidden decays such as to strange and antibottom quarks.

Already much work has been done in QCD studies. The running of $\alpha_{s}$ is now well established, and indirect evidence of the triple-gluon coupling has been seen in four-jet events. With several nearly independent ways to determine $\alpha_{s}$, studying a very large sample of hadronic decays from a single experiment, will allow stringent testing of QCD predictions to a degree impractical in previous experiments.

Heavy quark physics should also prove to be a rich field of study. Bottom and charm decay branching ratios have already been determined from the momentum and transverse momentum spectra of leptons in hadronic events. The precision of these measurements should improve steadily with increasing data and with better understanding of detector response. In addition, the precision vertex chamber should allow direct tagging of bottom and charm events via displaced vertices. The excellent momentum resolution of the central drift chambers will also permit tagging of primary strange quarks. The decay branching ratios determine directly the sum of the squares of the axial and vector coupling constants between the individual quarks and the $Z$. A slightly different combination of couplings has already been determined from the measurement of hadronic events with energetic final-state radiation. A third measure of quark couplings will be available soon through the forward-backward asymmetry of tagged heavy quark events. A weicome complica- 
tion to this tagging is $B^{0} \bar{B}^{0}$ mixing, which OPAL should be able to measure directly. In addition, fragmentation functions for $\mathrm{s}, \mathrm{c}$, and $\mathrm{b}$ quarks will be feasible using an assortment of quark-tagging techniques. All of these heavy-quark measurements should improve significantly with increasing integrated luminosity.

Although little affected by the $\mathrm{Z}$ resonance, two-photon physics benefits from the high center-of-mass energy of LEP and the high luminosities. Single-tagged events are presently being recorded, along with a reasonable sample of high-visible-energy untagged events. Within the next year, OPAL should be able to measure the photon structure function $F_{2}$ at $Q^{2}$ values inaccessible to previous $e^{+} e^{-}$experiments.

\subsubsection{Longer Term Prospects}

Our program at LEP and LEP II will be very productive for the next five years. The main effort for our group will be the maintenance and operation of the experiment, and data reduction and physics analysis. We are also contributing electronics expertise in the construction of the OPAL silicon vertex detector. In addition, there will be minor detector and electronic upgrades needed when LEP goes into multi-bunch operation for higher luminosity.

In the intermediate future, we are examining the possibility of participating in an experiment at the $B$ factory. The detailed study of the properties of the b-quark by measuring the $B \bar{B}$ mixing, the rare decay branching ratios, and CP violation is a consistent extension of our program in $e^{+} e^{-}$collisions.

In the longer range, we are looking into the physics opportunities at the very high energy colliders. Our participation has been sought in several detector R\&D projects, and the time is appropriate for becoming involved in such work. A postdoctoral research associate is needed to work with the existing members of the group on this project. 


\section{Bibliography}

[1] H. Aihara et al. (TPC/Two-Gamma collaboration), "Effects of soft and/or collinear gluon emission in $e^{+} e^{-}$hadronic events", Z. Phys. C44,357 (1989).

[2] H. Aihara et al. (TPC/Two-Gamma collaboration), "Exclusive production of $p \bar{p} \pi^{+} \pi^{-}$in photon-photon collisions", Phys. Rev. D40,2772 (1989).

[3] H. Aihara et al. (TPC/Two-Gamma collaboration), "Investigation of the Electromagnetic Structure of $\eta$ and $\eta$ ' Mesons by Two-Photon interactions", Phys. Rev. Lett. 64 ,172 (1990).

[4] H. Aihara et al. (TPC/Two-Gamma collaboration), "Measurement of the total hadronic cross section in tagged $\gamma \gamma$ reactions", Phys. Rev. D41 2667 (1990).

[5] H. Aihara et al. (TPC/Two-Gamma collaboration), "A search for new leptons with heavy neutrinos in $e^{+} e^{-}$annihilation at $\sqrt{s}=29 \mathrm{GeV}^{m}$, Submitted to 1989 Lepton/Photon Conference.

[6] H. Aihara et al. (TPC/Two-Gamma collaboration), "Study of two-photon production of $\rho \omega$ in $e^{+} e^{-}$interactions", Intended for Phys. Rev. Lett.

[7] H. Aihara et al. (TPC/Two-Gamma collaboration), "Measurement of the photon structure function $F^{(2)}$ between $10<Q^{2}<60 \mathrm{GeV}^{2 n}$, Intended for $Z$. Phys.

[8] H. Aihara et al. (TPC/Two-Gamma collaboration), "Inclusive $D^{\text {*t }}$ production in photon-photon collisions", Intended for Phys. Rev. D.

[9] H. Aihara et al. (TPC/Two-Gamma collaboration), "Charged hadron production in $e^{+} e^{-}$annihilation at sqrts $=29 \mathrm{GeV}^{n}$, Intended for Phys. Rev. D.

[10] H. Aihara et al. (TPC/Two-Gamma collaboration), "Jet production in photonphoton interactions at PEP", Submitted to 1989 Lepton/Photon Conference; intended for Phys. Rev. D.

[11] Mourad Daoudi, " $f_{2}^{\prime}(1525)$ Meson Production in Tagged Two-Photon Reactions", presentation at the Spring Meeting of the American Physical Society, Washington, D.C., 16-19 April 1990. 
[12] Elliott D. Bloom, "The PEP Program", July 1990 (unpublished). G. Gidal et al., "Report of the Ad-Hoc Committee on the Future of TPC at LBL", June 1989 (unpublished). The TPC/2 $\gamma$ Collaboration, "Response to the Report of the AdHoc Committee on the Future of TPC at LBL", June 1989 (unpublished).

[13] CERN-EP/90-27, Phys. Lett. B240 (1990) 497-512

[14] M. Greco, Phys. Lett. B177(1986) 97.

[15] M. Caffo, E. Remiddi, and F. Semeria, CERN 89-90.

[16] CERN-EP/90-48, Submitted to Zeitschrift für Physik.

[17] W. Bartel et al., Zeit. Physik C33, (1986) 23.

[18] CERN-EP/89-153, Physics Letters B235 (1990) 389.

[19] G. Kramer and B. Lampe, Zeit. für Physik C39, (1988) 101.

[20] M. Bengtsson and P. Zerwas, Phys Lett B208, (1988) 306.

[21] O. Nachtman and A. Reiter, Zeit. für Phys., C16 (1982) 45.

[22] M. Bengtsson, PITHA 88/12 (1988).

[23] E. Ma and J. Okada, Phys. Rev. Letters 41, 287 (1987)

[24] D. Karlen, Nucl. Phys. B289 (1987) 23.

[25] CERN-EP/90-29, Physics Lett. B241 (1990) 133-140.

[26] OPAL Collaboration, M. Z. Akrawy et al. Phys. Lett. B240 (1990) 497.

[27] OPAL Collaboration, M. Z. Akrawy et al. A search for acoplanar Pairs of leptons and jets in $Z^{0}$ decays; Phys. Lett. B240(1990)261

A search for Technipions and Charged Higgs Bosons at LEP; CERN-EP/90-38 Search for Exited Leptons at LEP; CERN-EP/90-49

Limits on Neutral Heavy Lepton Production from $Z^{0}$ Decays; CERN-EP/90-49

[28] Analysis of $Z^{0}$ Couplings to Charged Leptons; CERN-EP/90-81

[29] M. Dittmar, A Lepton Pair Analysis based on the Central Detector and the Barrel Lead Glass OPAL-OFFline-90/0033

[30] Particle Properties, Phys. Letters B204 (1988) 
[31] M. Dittmar and J. W. F. Valle; Heavy Singlet Neutral Leptons, Flavour and CP Violation on the Z Peak, to be published in Physics at the High Luminosity LEP

[32] CERN-EP/89-154, Phys. Lett. B236 (1990) 364.

[33] CERN-EP/89-176, Phys. Lett. B240 (1990) 250.

[34] CERN-EP/90-72, Submitted to Phys. Lett. B.

[35] K. Hagiwara et al., Z. Phys. C29 (1985) 115.

[36] CERN-EP/90-49, Submitted to Phys. Lett. B.

[37] CERN-EP/90-38, Submitted to Phys. Lett. B.

[38] S.L. Glashow, J. Iliopoulos, and L. Maiani, Phys. Rev. D2 (1970) 1285.

[39] P.W. Higgs, Phys. Lett. 12 (1964) 132.

[40] J. Ellis, M.K. Gaillard, and D.V Nanopoulos, Nucl. Phys. B106 (1976) 292.

[41] K. Hagiwara, R.D. Peccei, D. Zeppenfeld, and K. Hikasa, DESY86-058 (1986).

[42] A. Blondel, et al., Proceedings of the ECFA Workshop on LEP200, CERN 87-08, Eds. A. Böhm and W. Hoogland, Vol. I (1987) 120.

[43] F. Olness and W.K. Tung, Phys. Letters 179 (1986) 269.

[44] J. Bijnens, et al., Proceedings of the ECFA Workshop..., Vol I (1987) 49.

[45] M.L. Swartz, SLAC-PUB-5258 (1990).

[46] H.P. Nilles, Phys. Rept. 110 (1984) 1. H.E. Haber and G.L. Kane, Phys. Rept. 117 (1985). 117.

[47] J. Boucrot, et al., Proceedings of the ECFA Workshop..., Vol. II (1987) 312.

[48] T. Dombeck, et al., Phys. Letters B194 (1987) 591.

[49] X.Q. Lu, et al. LSND Proposal, Los Alamos Meson Physics Facility, 1989. 
TASK B: THEORY 


\section{TASK B: THEORY}

\section{Introduction}

The High-Energy Physics Theory Program at UC-Riverside has had another successful year. Since July 1989, there have been 16 completed research papers (UCRHEP-T42 to UCRHEP-T57). They are listed under Publications (Sec. 3) together with 20 other papers which were listed in last year's report as yet to be published but are now published. Of the 30 papers with definite publication information, 8 are in letter journals; 4 in Phys. Rev. Lett, 1 in Phys. Lett. B, and 3 in Mod. Phys. Lett. A. This is certainly indicative of our quality as well as productivity.

The Theory Group has consisted mainly of E. Ma (Professor), S. Rajpoot (Visiting Assistant Professor until December 1989), J. Pantaleone (Postgraduate Researcher), D. Ng and G. Wong (Graduate Student Research Assistants until August 1990). Dr. Rajpoot has left Riverside and is currently with Wayne State University in Detroit as Senior Research Associate. Mr. Ng and Mr. Wong are expected to finish their Ph.D. dissertations in August 1990. They will be postdoctoral research associates at the University of North Carolina, Chapel Hill and National Taiwan University, Taipei respectively beginning in September 1990. In the highly competitive environment of theoretical high energy physics, it is gratifying that they are both able to secure suitable employment in their field.

The Physics Department at UCR went through a recruitment search for a tenure-track junior faculty in theoretical high-energy physics this year. Out of nearly 100 applicants, Dr. Jose Wudka (then at UC-Davis) was selected and he will join the Theory Group beginning this Fall. Also, there will be two new research students, T.V. Duong and K. Mcllhaney.

In Sec. 2, the research activities of the Theory Group since July 1989 are discussed. In Sec. 3, there is a list of completed or published papers since July 1989. In Sec. 4, travel activities of the group are described and visitors to Riverside noted. In Sec. 5, there is a list of personnel and there are statements concerning their needs. In Sec. 6, the future research plans of Ma and Wudka are outlined. 
In sec. 7, Wudka's recent research activity is reviewed, in Sec. 8, the budget is given.

\section{Research Program}

Since July 1989, E. Ma, has completed 8 papers for publication. They are described briefly below.

The main focus of Ma's research continued to be in mass-generating radiative mechanisms for light quarks and leptons in renormalizable gauge field theories. In Paper \#29, the asymmetric left-right model proposed earlier in Phys. Rev. Lett. (\#7) is fully explored with details given for the scalar sector and phenomenological implications noted. In Paper \#30, the problem of $\mu$-e transitions in models of radiative lepton masses is pointed out and discussed in detail. Basically, the same mechanism which allows e and $\mu$ to obtain radiative masses will in general also induce $\mu \rightarrow$ e $\gamma$ and sometimes $\mu \rightarrow$ eee decays, with rates much higher than the present experimental upper bounds unless fine tuning of parameters is invoked. This makes such models unattractive unless $e$ and $\mu$ are distinguished by some unbroken symmetry. In Paper \#27, the lepton sector of a previous proposed model (E. Ma, Phys. Rev. Lett. 62, 1228 (1989)) is revised so that an odd-even exact symmetry serves to distinguish e from $\mu$, thus forbidding $\mu \rightarrow$ ey and $\mu \rightarrow$ eee, etc. In Paper \#32, assuming that only the top quark gets a tree-level mass due to electroweak gauge symmetry breaking, a model is presented where all other known quarks and leptons acquire radiative masses in a hierarchical manner. This is the first such model where the $3 \times 3$ charged-current quark mixing matrix is realistically generated without the fine tuning of parameters. In Paper \#34, the issue of CP nonconservation is brought up in supersymmetric models where the neutron electric dipole moment is typically 2 or 3 orders of magnitude above its experimental upper bound unless fine tuning is allowed. This situation is highly undesirable, especially in models of radiative masses where the absence of small parameters at tree level is their raison d'e tre. In this paper, a possible solution is presented which also gives the observed pattern of quark masses and mixing angles. Paper \#33 is based on a talk given by Ma at the American Physical Society Meeting of the Division of Particles and Fields in Houston, Texas (DPF 90) and will appear in the Proceedings. Paper \#36 is based on a talk given by Ma at the 13th Warsaw Symposium on Elementary Particle Physics in Kazimierz Dolny, Poland. It was one of two 
principal lectures (theory) at this Symposium and will appear in the Proceedings. Both of the above deal with radiative masses.

The other work by Ma (Paper \#22) concerns the meaning of lepton number in supersymmetric extensions of the standard model. Because of the presence of one Higgs superfield which transforms exactly the same as the usual left-handed lepton doublet, there are five generically distinct scenarios for assigning lepton numbers in addition to the conventional one, each with three possible variations. Existing experimental data constrain the values of various new couplings present in these models.

In the period July to December 1989, S. Rajpoot worked mostly on gauge extensions of the standard model. Papers \#21 and 23 dealt with gauging baryon and lepton numbers while Papers \#24 and 26 considered the gauging of quark and lepton weak interactions separately.

Since July 1989, J. Pantaleone completed a paper with P. Roy (\#25) which proposed the decay of a heavy quarkonium state into $\mathrm{Z}$ and Higgs boson as a means of distinguishing between whether the Higgs boson is a scalar or pseudoscalar particle. This can be done from a study of the angular distribution of the $\mathrm{Z}$ decay products. He also continued his work on neutrino oscillations and analyzed with T.K. Kuo (Paper \#31) recent Kamiokande solar neutrino data (which have energy spectrum information) to obtain new restrictions on the parameters for matter neutrino oscillations in the Sun. In Paper \#35, he and S. Pakvasa made the interesting discovery that line neutrinos from the Sun may show oscillations as a function of the Earth's distance to the Sun for a range of oscillation parameters inaccessible to any other experiment. These line neutrinos are expected to be observable with detectors now being proposed. If observed, these oscillations would be a direct and unambiguous proof of their existence independent of any assumed Solar Model.

Since July 1989, D. Ng participated in Papers \#22, 30 and 34. His Ph.D. dissertation is based on Paper \#22. He is working out all relevant processes for constraining and testing the various scenarios of lepton-number assignments in supersymmetry. He is expected to finish by August 1990 and become a Postdoctoral Research Associate at the University of North Carolina, Chapel Hill in September. 
Since July 1989, G.-G. Wong participated in Papers \#27, 29, and 30. His Ph.D. dissertation is based on Paper \#29. He is working out all the relevant details in the Asymmetric Left-Right Model for generating quark and lepton masses. He is expected to finish by August 1990 and become a Postdoctoral Research Associate at the National Taiwan University, Taipei in September.

\section{Publications}

The following list contains work published or completed by the Riverside Theory Group since July 1989.

1. UCRHEP-T17: "SU(3) X SU(2) X U(1) Model with Fritzsch Mass Matrices," S. Rajpoot, Phys. Rev. D年, 873 (1989).

2. UCRHEP-T18: "Purely Gluonic Soliton Solutions in the Leading-Logarithm Model," G.-H. Xu and B.R. Desai, Phys. Rev. D40, 620 (1989).

3. UCRHEP-T23: "New Supersymmetric Left-Right Model and Rare Decays," E. Ma and D. Ng, in Proc. of the Rare Decay Symposium, edited by D. Bryman et al. (World Scientific, Singapore, 1989), p. 495.

4. UCRHEP-T25: "Is e- $\mu-\tau$ Universality Violated?" E Ma and J. Pantaleone, in Proc. of Beyond the Standard Model, edited by K. Whisnant and B.-L. Young (World Scientific, Singapore, 1989), p. 417.

5. UCRHEP-T26: "Vector-Spinor Superalgebra and Goldstone Spin-3/2 Fermions," C. Pilot and S. Rajpoot, Int. J. Mod. Phys. A4, 4819 (1989).

6. UCRHEP-T27: "Distinct Hypercharge Sources for Quarks and Leptons," S. Rajpoot, in Proc. of Beyond the Standard Model, Edited by K. Whisnant and B.-L. Young (World Scientific, Singapore, 1989), p. 424.

7. UCRHEP-T28: "Radiative Quark and Lepton Masses in a Left-Right Gauge Model," E. Ma, Phys. Rev. Lett. 63, 1042 (1989).

8. UCRHEP-T29: "Neutrino Oscillations in Matter," T.K. Kuo and J. Pantaleone, Rev. Mod. Phys. 
61, 937 (1989).

9. UCRHEP-T30: "The Fourth Family as a Source of Radiative Masses for the First Two," E. Ma, in Proc. of the Second International Symposium on the 4th Family of Quarks and Leptions, Annals New York Academy of Sciences, $\underline{578} 323$ (1989).

10. UCRHEP-T31: "One-Loop Induced Fermion Masses and Exotic Interactions in a Standard-Model Context," E. Ma, D. Ng, J. Pantaleone, and G.-G. Wong, Phys. Rev. D40, 1586 (1989).

11. UCRHEP-T32: "The ז-Lepton Lifetime Discrepancy as Evidence for the 4th Family," S. Rajpoot, in Proc. of the Second International Symposium on the 4th Family of Quarks and Leptons, Annals New York Academy of Sciences, $\underline{578}, 405$ (1989).

12. UCRHEP-T33: "Heavy Majorana Neutrino Production," E Ma and J. Pantaleone, Phys. Rev. D 40 , 2172 (1989).

13. UCRHEP-T34: "Radiative quark and Lepton masses," E. Ma, in Proc. of 12th International Workshop on Weak Interactions and Neutrinos, Nucl. Phys. B (Proc. Suppl.) 13, 603 (1990).

14. UCRHEP-T35: "Radiative Hierarchy of Majorana Neutrino Masses," K.S. Babu and E. Ma, Phys. Lett. B228, 508 (1989).

15. UCRHEP-T36: "CP Violation in Models with Chiral Color," X-G. He and S. Rajpoot, Phys. Rev. Lett. 63,486 (1989).

16. UCRHEP-T37: "Radiative Mechanisms for Generating Quark and Lepton Masses: Some Recent Developments," K.S. Babu and E. Ma, Mod. Phys. Lett A4, 1975 (1989).

17. UCRHEP-T38: "Electroweak Interactions with Gauged Baryon and Lepton Numbers," S. Rajpoot, Phys. Rev. D40, 2421 (1989).

18. UCRHEP-T39: "Lepto-Quark Matter," S. Rajpoot, Z. Phys. C45, 681 (1990).

19. UCRHEP-T40: "The Construction of Left and Right Handed Chiral Superfield with $(1,1 / 2)+$ $(1 / 2,1)$ Supersymmetry," C. Pilot and S. Rajpoot, Int. J. Mod. Phys. A (in press). 
20. UCRHEP-T41: "Identifying $\tau$ as a Nonleptonic Superparticle," E. Ma and P. Roy, Phys. Rev. D41, 988 (1990).

21. UCRHEP-T42: "Left-Right Symmetry with Baryon- and Lepton- Number Conservation as Gauge Symmetries," S. Rajpoot, Phys. Rev. D40, 3795 (1989).

22. UCRHEP-T43: "Assignment of Lepton Numbers in Supersymmetry," E. Ma and D. Ng, Phys. Rev. D41, 1005 (1990).

23. UCRHEP-T44: "Anomaly-Free Left-Right Symmetric Models with Gauged Baryon and Lepton Numbers," X.-G. He and S. Rajpoot, Phys. Rev. D41, 1636 (1990).

24. UCRHEP-T45: "Alternative Ununified Standard Model," S. Rajpoot, Phys. Rev. D41, 995 (1990).

25. UCRHEP-T46: "Heavy-Quarkonium Decay into $Z+$ Higgs Boson as a Scalar/Pseudoscalar Discriminant," J. Pantaleone and P. Roy, Phys. Rev. Lett. 64, 264 (1990).

26. UCRHEP-T47: "Completely Ununified Standard Model," S. Rajpoot, Phys. Rev. D41, 1706 (1990).

27. UCRHEP-T48: "Dichotomy of leptons: Radiative Masses and Rare Decays," E. Ma and G.-G. Wong, Phys. Rev. D41, 992 (1990).

28. UCRHEP-T49: "Sign of the Neutron-Proton Mass Difference in an SU(2) x U(1) Supersymmetric Toy Model: A Possible Scenario for Solving the Old Puzzle," B.,R. Desai and G.-H. Xu, Phys. Rev. D41, 2214 (1990).

29. UCRHEP-T50: "Asymmetric Left-Right Model for Generating Radiative Quark and Lepton Masses," E. Ma and G.-G. Wong, Phys. Rev. D41, 953 (1990).

30. UCRHEP-T51: "Muon-Electron Transitions in Models of Radiative Lepton Masses," E. Ma, D. Ng, and G.-G. Wong, Z. Phys. C (in press).

31. UCRHEP-T52: "Solar Neutrino Observations and Neutrino Oscillations," T.K. Kuo and J. Pantaleone, Phys. Rev. D (in press).

32. UCRHEP-T53: "Hierarchical Radiative Quark and Lepton Mass Matrices, " E. Ma, Phys. Rev. Lett. 64, 2866 (1990). 
33. UCRHEP-T54: "Radiative Generation of Quark and Lepton Masses,": E. Ma, in Proc. of DPF90 (to be published).

34. UCRHEP-T55: "CP Nonconservation in Supersymmetry with Radiative Quark Masses," E. Ma and D. Ng, submitted to Phys. Rev. Lett.

35. UCRHEP-T56: "Direct Probes of Neutrino Properties Using Solar Neutrino Lines," S. Palvasa and J. Pantaleone, submitted.

36. UCRHEP-T57: "Quark Mass Problem," E. Ma, in Proc. of 13th Warsaw Symposium on Elementary Particle Physics (to be published).

\section{Travel and Consultants}

E. Ma attended Hadron '89 (Ajaccio, France) in September 1989 with funds provided by the University. In January 1990, he attended the APS Meeting of the Division of Particles and Fields (Houston, Texas) and gave a talk. He then visited the University of Texas, Austin and gave a seminar. In February, he attended the Workshop on Thinking About the Top Quark at the University of California, Santa Barbara. In March, he attended the First International Symposium on Particles, Strings and Cosmology (Boston, Massachusetts), and also gave a seminar at the University of Massachusetts, Amherst. In April, he visited the University of California, Davis and gave a seminar. In May, he attended the 13th Warsaw Symposium on Elementary Particle Physies and delivered one of the principal lectures. In August, he is planning to attend the 25th International Conference on High Energy Physics in Singapore where he is responsible for organizing one of the parallel sessions.

J. Pantaleone attended the SLAC Summer Institute and Topical Conference in July 1989. He also attended Neutrino '90 (Geneva, Switzerland) in June 1990 and visited the Gran Sasso underground laboratory in Italy.

Visitors who gave high-energy theory seminars/colloquia include K.S. Babu (Maryland), K. Choi (Carnegie-Mellon), R. Flores (Minnesota), F. Halzen (Wisconsin-Madison), R. Volkas (Melbourne), and J. Wudka (UC-Davis). 


\section{Personnel and Needs}

Two faculty members are now covered by this proposal: E. Ma (Professor) and J. Wudka (Assistant Professor), for whom 2.5 months of summer salary are requested. J. Pantaleone will continue to be Postgraduate Researcher (100\% time) and another postdoctoral research associate ( $50 \%$ time) is requested to match the University's funding (also $50 \%$ time) for such a position. Two new graduate students, T.V. Duong and K. Mcllhaney, will be joining the Theory Group as Research Assistants.

Two foreign trips are planned, at about $\$ 3,000.00$ each, to conferences such as the Lepton-Photon Symposium and the Weak-Interaction Workshop, etc. Four domestic trips are planned, at about $\$ 1,500.00$ each, to conferences such as APS meetings and various other workshops and summer institutes yet to be announced. About $\$ 2,000.00$ is requested for bringing in consultants to work on research projects of mutual interest.

As foreseen in the last proposal, we have been able to add a junior faculty member to the Physics Department in the area of Theoretical High Energy Physics beginning in Fall 1990. We are fortunate to have recruited Dr. Jose Wudka and his presence will certainly enhance the Theory Program at UCR which has already a very strong record since the intital funding of this Task by DOE in February 1988. Continuing support by DOE and at a level compatible with the Group's activities is vital for its future success.

\section{Future Research Plans}

In the immediate future, E. Ma plans to work on three projects:

(A) With student T. V. Duong, he will study in detail the model of Paper \# 32 "Hierarchical radiative quark and lepton mass matrices" to extract all relevant phenomenological information regarding the exotic scalar bosons which are needed to generate the light quark and lepton masses. This will help define the problem of looking for experimental evidences of such radiative mechanisms for understanding masses and mixing angles in present and future high-energy accelerators. 
(B) With student K. Mcllhaney, he will also study the specific model proposed in Paper \#34 "CP nonconservation in supersymmetry with radiative quark masses" for generating realistic quark mass matrices from scalar quarks. The simple Fritzsch ansatz for quark mass matrices is now on the verge of being ruled out by the experimental data on $m_{t}$ and $V_{u b} / V_{c b}$ However, if a similar restriction is put on the scalar-quark mass matrices by the help of a discrete symmetry, the induced quark mass matrices are only approximately of the Fritzsch form and will likely be consistent with all present data. A detailed study will quantify the above and perhaps correlate the allowed values of $m_{t}$ versus $V_{u b} / N_{c b}$, to be confronted with data in the future.

(C) From the $\mathrm{e}^{+} \mathrm{e}^{-} \rightarrow \mathrm{b}$ b forward-backward asymmetry at TRISTAN and the direct decay $\mathrm{Z} \rightarrow$ b b at LEP, we now know for certain that $b_{\mathrm{L}}$ has weak isospin -1/2. It appears thus that the $t$ quark must exist so that $t_{L}$ has weak isospin $+1 / 2$. However, it is still conceivable that the t quark may not exist. For example, if all quarks are composite, the dynamical mechanism which creates them may be such that $u, d, s, c$ and $b$ are bound but not $t$ ! In a gauge theory, the absence of $t$ may be achieved by a nonlinear (but gauge-invariant) constraint such that in the physical $U$ gauge, $t_{L}$ simply disappears. The basic idea is not even new; see N. P. Chang and E. Ma, Phys. Rev. D7, 3808 (1973) and L D. Faddeev, Sov. Phys. Dokd. 18 382 (1973). The consequences of this "crippled" standard model will be studied and loop processes will be used to find the mass scale at which this effective theory breaks down. If the $t$ quark is not found within the next few years so that $m_{t}$ exceeds its present upper bound of about $200 \mathrm{GeV}$ predicted by the standard model, and no new physics is observed, then this unorthodox idea may well be correct.

In the following a brief summary of Wudka's intended activities for the near future are described. Some of the projects described below are almost completed, others will require more work. 
Unitarity (with J. F. Gunion and H. Haber).

It is well known that, aside from some special cases, a model of massive gauge bosons, scalars and fermions must be a spontaneously broken gauge theory. [J. M. Cornwall, et.al., Phys. Rev. D10 (1974) 1145.]

In this collaboration we re-examined this result, corrected several minor errors in the existing published work, and provided a useful compilation of the sum rules required by imposing this unitary condition on an arbitrary phynomenological model of massive gauge bosons and scalars. The results are presented so as to be "user friendly" toward the experimental community. The main idea behind this work is that, once some experimental information about the scalar sector is obtained, these sum rules can be used to determine whether the observed particle content and coupling strengths can be accommodated within a consistent model. Were this not the case, strong theoretical reasons would require the existence of more scalar particles, or further interactions. For example, we rederive the result that the existence of a W Z H coupling requires the presence of doubly charged scalars. Moreover, our sum rules can be used to set limits on this type of undiscovered physics. This work is almost completed.

Exotic Models (with J. F. Gunion and R. Vega).

As a continuation of the work [\#1 in Sec. 7] for the inclusion of a scalar triplet in the standard model, the authors have studied the naturalness issues which arise in this theory. The model, as originally constructed, was required to possess a larger symmetry group than the usual gauge symmetry SU(2) $x \mathrm{U}(1)$. In correspondence to the standard model, the scalar potential was assumed to be $S U(2)_{L} \times S U(2)_{R}$ invariant, which preserves the phenomenologically successful relations $\rho=1$. This construction, however, is inconsistent when radiative corrections are calculated. Since electromagnetism violates the symmetry of the scalar sector, new infinities arise which cannot be compensated by redefining any of the original couplings or fields. Therefore, to be able to use this model, the original potential must be generalized; in this case the problem with 
the infinities disappears, but the $p$ parameter is no longer predicted. The issue, just as in the standard model - albeit more severe -, is one of naturalness: we are forced to fine tune the parameters in the potential to agree with experimental results. To study this in detail we performed the renormalization of the model up to one loop, and determined the conditions required to satisfy $p=1$. This also affects the one-loop induced decays of several scalars, in particular, the scalars which have no tree level couplings to fermions will decay into fermion pairs via one-loop diagrams. These decays are calculated and are found to be significant when the scalar mass is below $2 m_{W}$. The pheonomenology of the model is reexamined when affected by these results. This work is almost completed.

Screening Theorem (with M. Einhorn).

As a continuation of our published work, we are now considering the generalizations of the screening theorem: given any theory with spontaneously broken symmetries containing heavy scalars, when are the leading radiative corrections (in the mass of the scalars) not observable?

This type of study is interesting on two counts. First it provides a guideline as to which models will satisfy the constraint of not producing unacceptably large radiative corrections to the vector boson masses. Second, it provides useful insight into the strong constraints the symmetries impose on the observables of a model.

Our (preliminary) answer to the above question lies both in symmetries of the model, and in the pattern of symmetry breaking: if the symmetry group of the scalar potential is $G$ and is broken down to $H$, then the conditions for screening to occur is that, first, each presentation of $\mathrm{G}$ (realized in the model) should contain a representation of $\mathrm{H}$ at most once; and, second, that two different representations of $G$ (realized in the model) should have no representations of $H$. in common. This is certainly satisfied in the standard model where $G=O(4)$ and $H=O(3)$. We are now in the process of illustrating the analysis with several examples. 


\section{Tensor Operators and the Adiabatic Approximation}

In this work I study a general mechanical system whose symmetry is broken explicitly by the presence of tensor operators in the Hamiltonian. This system presents a natural generalization of the classical examples where the adiabatic phases were first illustrated (such as the spin 1/2 system in a magnetic field, of an atom in an electric field). The purpose of the investigation is to provide an explicit expression for the adiabatic phases for a large class of Hamiltonians, and therefore enlarge the range of models where these phases have been studied, and may possibly be measured.

For the models studied many formulas can be obtained explicitly using simple group theoretical methods, without the need of powerful machinery such as fibre bundles. I am now in the process of completing the formalism and compiling several illustrative examples.

\section{Solar and Stellar Neutrinos}

A collaboration with J. Vidal has been started which intends to give a rather detailed account of the magnetic-flip approach to the solar neutrino problem. The issues to be investigated are i) the various mechanisms of magnetic moment generation. ii) The careful taking into account of a realistic description of the Solar magnetic field, supported, as far as possible, by observational data. iii) The details of the quantum mechanical evolutions of the neutrinos through the Sun; while previous analyses have been based on the adiabatic or semiclassical approximations, this may not be a valid procedure in a realistic Solar model, therefore the problem requires more analysis. As a longer term goal we also intend to study the neutrino production in supernovae and the early universe, (this last project is still in the planning stages).

In the next five years, with new data coming from TEVATRON and LEP, theoretical research in particle physics will be more phonomenology-oriented. The research activity of the UCR theory group has always been to push hard against our present theoretical understanding of experimentally verifiable issues, with new ideas and models. This will undoubtedly continue as its main focus. Research in the kind of new physies accessible with the SSC will also be carried out. 


\section{Recent Research Activity (T. Wudka)}

1. Higgs Triplets In the Standard Model (J. F. Gunion, R. Vega and J. Wudka).

Much attention has been paid to the extensions of the Standard Model in which the scalar sector is populated by two or more doublets. Still the remaining manifold of possibilities, where the scalar sector carries more complicated representations of the gauge group, has been mostly ignored. In this work we studied the phenomenology and theoretical structure of a model, originally proposed by Georgi and collaborators (Nucl Phys. B262 (1985), 463), and later studied by Chanowitz and Golden (Phys. Lett B165 (1985), 105), where the scalar sector contains a doublet and a triplet.

In the present work, the phenomenology of the model is considered in detail. The limits on the parameters are studied and the possible decays of the scalars are determined, together with the corresponding possibilities for detection at future and present colliders.

A peculiar characteristic of this model is the presence of scalars which have no tree-level couplings to fermions, so that the only allowed decays are via vector bosons. If the mass of these objects lies below $2 \mathrm{~m}_{\mathrm{W}}$ the possibility arises for the existence of long lived, charged scalars. The strong constraints on such particles which will follow from the near-future collider experiments will put severe constraints on this model. The decays of the remaining scalar excitations, which do couple to fermions, is more standard. Though, because of the increased particle content, the specifies are quite involved.

The model, as originally constructed, was required to satisfy $p=1$ as a tree level relation. This constraint restricts considerably the types of interactions in the scalar sector and renders the model unsuitable for calculating radiative corrections. If the constraint on the parameter is relaxed then these problems disappear but a naturalness problem arise instead: fine tuning is required to comply with experimental data. These issues are touched upon in this paper, but are dealt at length in a upcoming publication (described in Sec. 6 below). 


\section{Adiabatic Evolution of Quantum Mechanical Systems (J. Vidal and J. Wudka).}

The purpose of this paper is to study the systematic corrections to the adiabatic approximation under various circumstances. To this end the formalism of Berry is restated in the language of the time evolution operator for an arbitrary quantum mechanical system. It is then shown that all the corrections to the adiabatic approximation can be obtained by solving a of Schrödinger-like equation.

The formalism is then applied to the case where the system interacts with a rapidly varying external field. In this case a classical method (developed originally by Kapitsa) can be applied, and the resulting equations are much easier to deal with than the original ones. Several examples of the usefulness of the approach are provided. In particular, the case of a spin 1/2 particle interacting with a strongly oscillating magnetic field is studied in detail. For this system it is seen that resonance phenomenon appears as a result of the strong oscillations, and this produces dramatic effects, such as strong spin up-spin down transitions, which would be absent otherwise.

We also study the possibility of "stabilizing" the adiabatic approximation, in the same way a classical particle in an unstable potential is stabilized when rapid oscillations are present (parametric resonance phenomena); but found no indications that this classical effect is perpetuated within the quantum adiabatic approximation.

\section{Screening of Heavy Higgs Radiative Corrections (J. Wudka).}

This paper is a simplified version of another work, performed in collaboration with $\mathbf{M}$. Einhorn (Phys. Rev. D $\underline{39}$ (1989), 2758). The subject of both these papers is the screening phenomena first described by Veltman (Acta Phys. Pol. B $\underline{8}$ (1977), 475). This effect consists in that the standard-model radiative corrections, do not have the (naively) expected strong dependence on the mass of the Higgs $m_{M}$, when $m_{H}>m_{W}$ : the effects of the large Higgs mass are "screened." This result is important because, for example, it forbids an $\alpha m_{B}^{2}$ dependence in 
the leading radiative corrections (to one loop) to the vector boson masses. This is turn implies that the radiative effects originating in the scalar sector will be difficult to observe, in contraction to simple dimensional analysis arguments.

In this study we show that the screening effect is a natural result of the renormalization program and, in fact, that it can be extended to all orders in perturbation theory (the original calculations were limited to one and two loops). An interesting technical point is that the presence of an extra symmetry in the scalar sector (the custodial SU(2)) is crucial in the proof of the theorem. In fact, more recent calculations suggest that this type of symmetries is, in fact, essential for screening to occur.

We also devised a gauge--fixing condition which has all the advantages of the 't Hooft gauges, together with those of the background field gauge. This gauge fixing was subsequently shown to be useful in other areas, such as detailed studies of the equivalence theorem.

4. External Sources for Topologically Massive Gauge Fields (J. Wudka and J. L. Diaz-Cruz).

In this work we study the solutions to the classical Yang-Mills equations in three (Euclidean) dimensions, with the inclusion of a Chern-Simons (or topological mass) term, in the presence of external sources. This type of studies have been performed in the past for 4-dimensional YangMills theories, and have produced interesting results (J. Mandula, Phys. Rev D14 (1976), 3497; R. Jackiw, L Jacobs and C. Rebbi, Phys. Rev. D20 (1979), 474). Also, previous studies have appeared for free topological massive gauge theories (E D'Hoker and L. Vinet, Ann. Phys. 162 (1985), 413), but this is the first investigation on the excitations produced by external sources. This type of investigations, aside from clarifying the mathematical properties of the corresponding nonlineal equations, also provide some insight into their behavior under extreme circumstances (where matter behaves classically but the gauge fields are quantized). We consider both the perturbative (Abelian and non-Abelian) solution, as well as the full equation within the radial ansatz. Though we did not attempt a systematic classification of the solutions, a rather complete picture did 
emerge.

We also considered the stability of the solutions by studying the effects of small perturbations. A peculiar feature of all the solutions obtained is that they are never true minima of the action functional, so that they are all unsuitable for a semiclassical expansion. The corresponding destabilizing modes were identified and, though they include the ones corresponding to gauge transformations, they are not exhausted by them.

In the case of the non-perturbative solutions within the spherical ansatz, we considered both the usual source coupling $A \cdot J$, as well as the coupling of the source to a (gauge invariant) Wilson loop W: $\mathrm{J} \cdot \mathrm{W}$. In both cases the solutions are characterized, for a delta function source, by three parameters: The strength of the source, its position, and the "angular momentum" of the solution (the equations of motion separate and, in the usual way, a separation parameter appears). To complete the characterization of the solutions we determined (numerically) the regions in this parameter space that corresponds to finite-action solutions.

\section{A Comment on the Born-Oppenheimer Approximation (J. Wudka)}

In this short note the relation between Berry's work and the well know Born-Oppenheimer (BO) approximation are clarified. The connection is not immediate because each of these approximations corresponds to an expansion in different parameters. In fact, one must include higher order correction to Berry's expressions in order to derive the $\mathrm{BO}$ formulae. This correction is obtained using the techniques of short distance expansion common in field theory. The accuracy of the BO approximation is also determined.

\section{Topological Phases and the Solar Neutrino Problem (J. Wudka and J. Vidal).}

The Solar neutrino puzzle has interested theorists in recent years since it may signal new properties of the neutrino system which cannot be investigated using Earth-bound experiments. Within this context, one of the proposed explanations of the Solar neutrino problem is that the Solar magnetic field induces left-right transitions in the neutrinos, and this reduces the flux of left 
handed electron neutrinos (which is what has been measured). The problem with this assumption, as originally stated, is that it requires a large magnetic moment (of the order of $10^{-10}-10^{-11}$ in units of Bohr magnetons) which is on the verge of being disallowed by experiment. Moreover strong assumptions were made regarding the coherent organization of the Solar magnetic field which are, at present, not accessible to experimental verification.

In view of these facts a new approach is proposed in this paper which uses both the correct version of the adiabatic approximation, as stated by Berry; and a realistic (and experimentally verified) model of the Solar magnetic field. Within this scenario it is found that the observed flux reduction can be obtained for much smaller magnetic moments (of the order ${ }^{-13}$ in units of Bohr magnetons). The corrections to the adiabatic approximation were also investigated, and the results were found not to be affected provided certain kinds of correlations are absent. This assumption is currently under investigation.

\section{Publications}

1. "Higgs triplets in the standard model" (with J. F. Gunion and R. Vega), Phys. Rev. D42 (1990).

2. "Adiabatic Evolution of Quantum Mechanical Systems (with J. Vidal), Univ. of Calif. at Davis reprint UCD-89-22, submitted to Nucl. Phys. B.

3. "Screening of Heavy Higgs Radiative Corrections," contributed paper to the International Europhysics Conference on High Energy Physics, Madrid (Spain), Sept 6-13, 1989.

4. "External Sources for Topologically Massive Gauge Fields," (with J. L. Diaz-Cruz) Phys. Rev. D41, 581 (1990).

5. "A Comment on the Born-Oppenheimer Approximation," Phys. Rev. D41 712 (1990).

6. Topological Phases and the Solar Neutrino Problem," (with J. Vidal) Univ. of Calif. at Davis reprint UCD-88-40, submitted to Phys. Rev. Lett. 


\section{Talks}

1. "Solar Neutrinos and the Sun's Magnetic Field," Univ. of Calif., Riverside, Jan. 1990.

2. "On the Adiabatic Approximation," Universidad de Valencia, Valencia, Spain, Sept. 1989.

3. "Screening of Heavy Higgs Radiative Effects," Univ. Autónoma de Barcelona, Barcelona, Spain, Sept. 1989.

4. "Introduction to Berry's Phase," Instituto de Física, Universidad Nacional Autónoma de México, México, D. F., June 1989.

5. "Effects of a Very Massive Higgs," Univ. Nacional Autónoma de México, México, D. F., June 1989.

6. Topological Phases and the Solar Neutrino Problem," LBL, Jan. 1989.

\section{Courses}

Topological Phases: Introduction and Applications," set of six lectures given at the CINVESTAV Summer School, CINVESTAV del IPN, June 1989. 
A proposal to upgrade our computing infrastructure is being separately submitted to DOE. Here we describe briefly the current facilities and the improvements which are needed: more disk storage and $8 \mathrm{~mm}$ tapes for workstations and additional power and flexibility in the control "boot server".

The VAX/VMS system has been the core of the High Energy Physics analysis system at UCR for over ten years. Both of the current major collaborations (DZERO at FNAL and OPAL at CERN) support and distribute standard analysis and Monte Carlo software for VAXen. The Physics VAXCluster is a department resource, with support funding through the DOE tasks, and the IGPP Space Physics group. The dominant use is (and will remain) with the high energy experimental and theoretical groups. The Riverside campus has provided additional support for instructional use, and access to electronic mail and standard software. This university support is at the level of $\$ 50 \mathrm{~K}$ per year. Operations are contracted from the campuswide Computing and Communications group. They provide system management, maintenance contacts, software installation, and routine backups.

The VAXCluster consists of a central boot server (VAX 3500), and 11 satellite VAX Stations. Disk capacity exceeds 9 GigaBytes, and magnetic tape drives in both 9track and $8 \mathrm{~mm}$ format are available. Both DZERO and OPAL have adopted the $8 \mathrm{~mm}$ digital video tape as a standard for data and DST distribution. These units can be attached directly to the VAX stations allowing processing to and from tape, and backup of data on the local disks of the stations.

The present disk capacity does not meet the demands of several operating experiments concurrently. There are inadequate facilities for staging data samples to disk, and too few tape drives to provide the data. Without enhancement to disk and tape systems, the substantial computing capacity of the system cannot be fully exploited. 
At present the two major experimental groups have disk space for standard programs, an additional 1.2GB each for long term data storage and temporary working space combined. The disk system should be upgraded to allow a total of about $5 \mathrm{~GB}$ of data and temporary disk for each major project area, for a total of ten GB.

The present microVAX 3500 central node can just handle the satellite systems, and modest time-sharing use. As more stations are added to the cluster a more powerful system is needed. The VAX 6410 system provides 7.0 VUPS of power, up to $256 \mathrm{MB}$ of central memory, and the VAXBI I/O channel for additional mass storage. The 6410 can be subsequently upgraded with additional processors, or a vector processor and would replace the existing VAX 3500 as the cluster boot server, file server, and central time-sharing system. 